Development of a Generalized Methodology for Soil-Structure Interaction Analysis Using Nonlinear Time-Domain TechniquesNEAMS Program, DOE Office of Nuclear Energy (NE-41)

J. M. Solberg, Q. Hossain, J. A. Blink, S. R. Bohlen, G. Mseis, H. Greenberg

April 26, 2013 
This document was prepared as an account of work sponsored by an agency of the United States government. Neither the United States government nor Lawrence Livermore National Security, LLC, nor any of their employees makes any warranty, expressed or implied, or assumes any legal liability or responsibility for the accuracy, completeness, or usefulness of any information, apparatus, product, or process disclosed, or represents that its use would not infringe privately owned rights. Reference herein to any specific commercial product, process, or service by trade name, trademark, manufacturer, or otherwise does not necessarily constitute or imply its endorsement, recommendation, or favoring by the United States government or Lawrence Livermore National Security, LLC. The views and opinions of authors expressed herein do not necessarily state or reflect those of the United States government or Lawrence Livermore National Security, LLC, and shall not be used for advertising or product endorsement purposes.

This work performed under the auspices of the U.S. Department of Energy by Lawrence Livermore National Laboratory under Contract DE-AC52-07NA27344. 


\title{
Development of a Generalized Methodology for Soil-Structure Interaction Analysis Using Nonlinear Time-Domain Techniques \\ NEAMS Program, DOE Office of Nuclear Energy (NE-41)
}

\author{
Jerome M. Solberg \\ Quazi Hossain \\ James A. Blink \\ Steven R. Bohlen \\ George Mseis \\ Harris R. Greenberg
}

Report \# : LLNL-TR-635762

$5 / 3 / 2013$

\begin{abstract}
A generalized time-domain method for Soil-Structure Interaction Analysis is developed, based upon an extension of the Bielak Method. The methodology is combined with the use of a simple hysteretic soil model based upon the Ramberg-Osgood formulation and applied to a notional Small Modular Reactor. These benchmark results compare well with those obtained by using the industry-standard frequencydomain code SASSI. The methodology provides a path forward for investigation of other sources of nonlinearity, including those associated with the use of more physically-realistic material models incorporating pore-pressure effects, gap opening/closing, the effect of nonlinear structural elements, and 3D seismic inputs.
\end{abstract}


Lawrence Livermore National Laboratory is operated by Lawrence Livermore National Security, LLC, for the U.S. Department of Energy, National Nuclear Security Administration under Contract DE-AC5207NA27344

This document was prepared as an account of work sponsored by an agency of the United States government. Neither the United States government nor Lawrence Livermore National Security, LLC, nor any of their employees makes any warranty, expressed or implied, or assumes any legal liability or responsibility for the accuracy, completeness, or usefulness of any information, apparatus, product, or process disclosed, or represents that its use would not infringe privately owned rights. Reference herein to any specific commercial product, process, or service by trade name, trademark, manufacturer, or otherwise does not necessarily constitute or imply its endorsement, recommendation, or favoring by the United States government or Lawrence Livermore National Security, LLC. The views and opinions of authors expressed herein do not necessarily state or reflect those of the United States government or Lawrence Livermore National Security, LLC, and shall not be used for advertising or product endorsement purposes 


\section{Table of Contents}

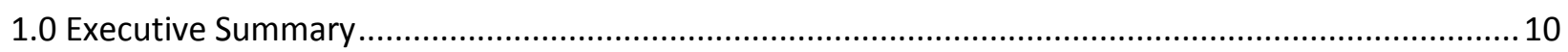

1.1. The Need for a Generalized Nonlinear Time-Domain SSI Analysis Methodology ......................11

1.2 Review of Current Nonlinear Time-Domain SSI Analysis Methods........................................... 12

1.3. Development of LLNL's Nonlinear Time-Domain SSI Analysis Methodology .....................12

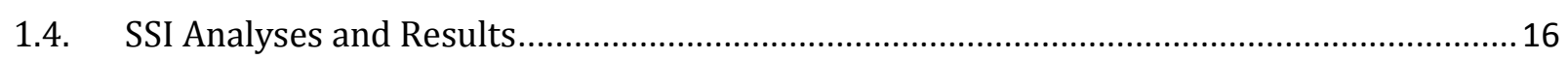

1.5. Development of Advanced Soil Models for SSI Analyses .................................................... 25

1.6. Development of Three-Dimensional Seismic Input Motions .................................................. 25

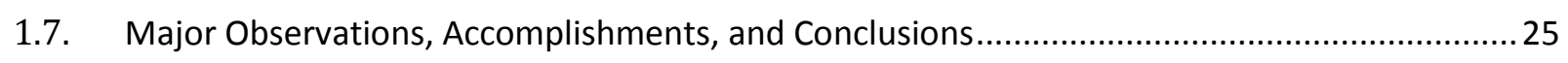

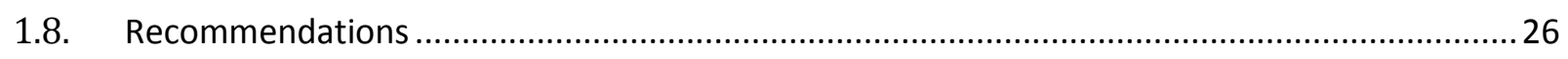

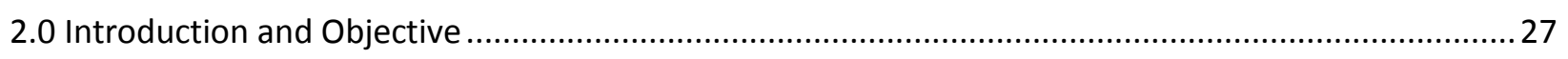

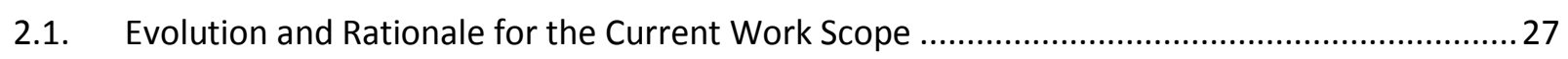

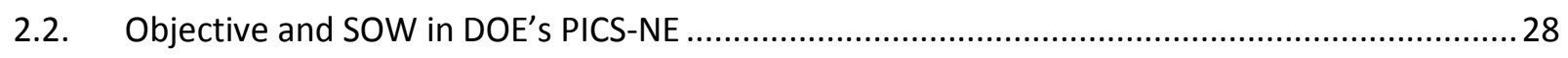

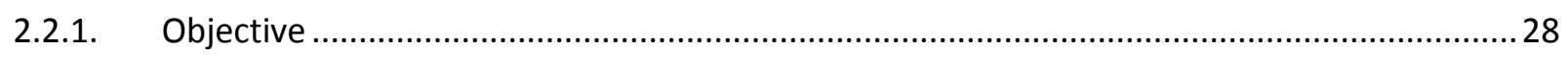

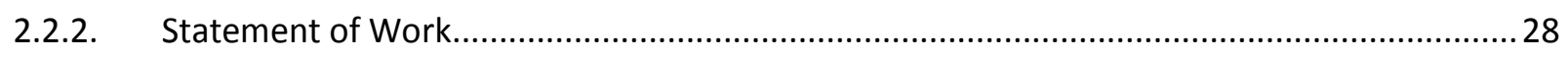

2.3. Workshop Organization, Summary and Recommendations..................................................28

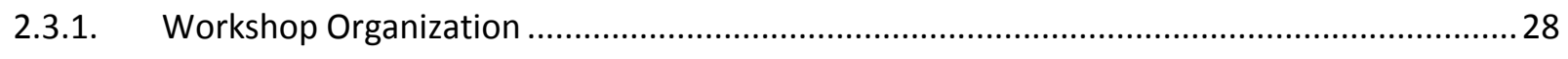

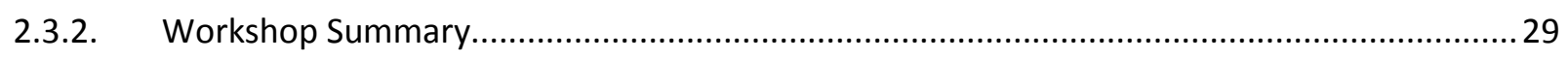

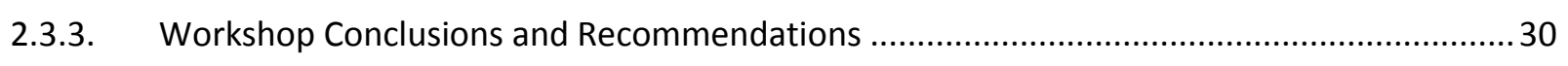

2.3.3.1. Nonlinearities Associated with the Interface between the Structure and the Soil .............31

2.3.3.2. The Use of Arbitrary, Spatially-Distributed Wave Fields as Seismic Input......................... 32

2.3.3.3. Nonlinear Hysteretic Models of Structural Elements .................................................. 33

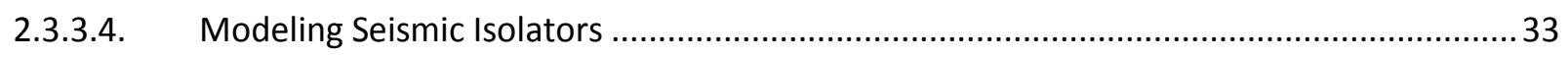

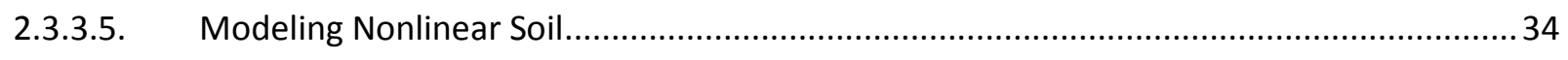

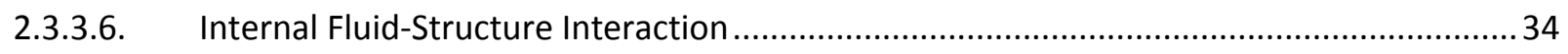

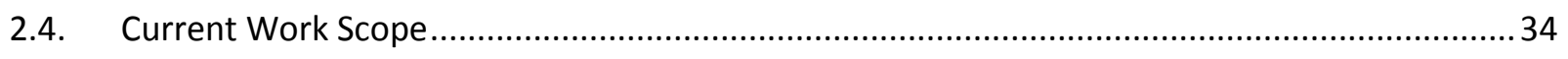

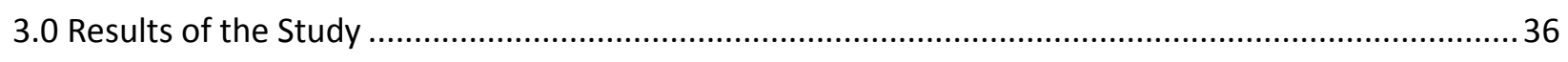

3.1. Development of a Finite Element Model for a Typical Modular Reactor Building ....................36

3.1.1. Reactor Building Configuration and Development of the Finite Element Model...................36

3.1.2. Eigenvalue Analysis of the Reactor Building ................................................................. 47

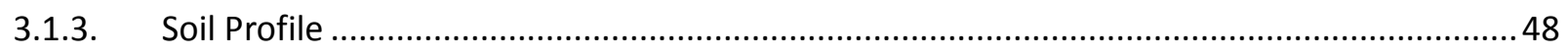


3.2. Development of Finite Element Technology for SSI analysis ...............................................53

3.2.1. A Description of the Time- and Frequency-Domain Finite Element Methods.......................53

3.2.2. Stiffness (Moduli) representation in SASSI versus Nonlinear Time Domain Analyses.............56

3.2.3. Representation of Damping (Dissipation) in SASSI versus Nonlinear Time-Domain Analyses61

3.2.4. Boundary Conditions for Seismic Analyses in Semi-Infinite media.......................................72

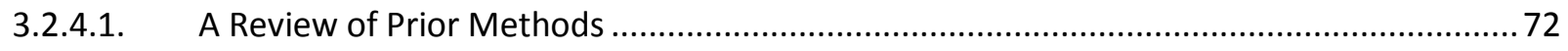

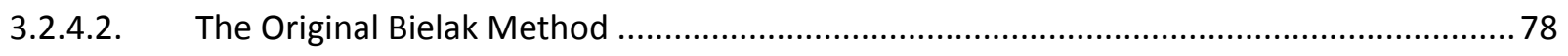

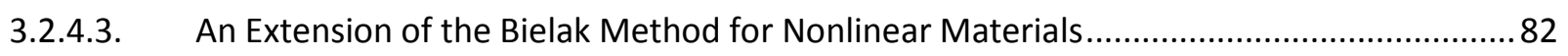

3.2.4.4. The Modified Bielak Method - Gravitational Preload and Contact..................................87

3.2.4.5. The Modified Bielak Method - 3D input motions ......................................................... 88

3.2.4.6. The Modified Bielak Method - Summary ..................................................................... 92

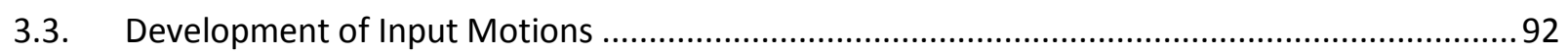

3.3.1. Derivation of 1-D input motions for typical earthquakes at the $0.05 \mathrm{~g}, 0.2 \mathrm{~g}, 0.5 \mathrm{~g}$, and $0.9 \mathrm{~g}$ levels 92

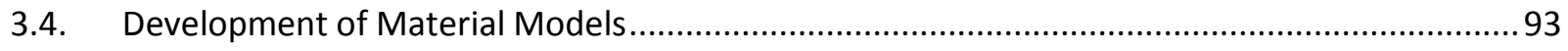

3.4.1. Development Equivalent-Linear Material Coefficients using CARES for use in SASSI ...........93

3.4.2. Development of Ramberg-Osgood (RO) coefficients using RAMBO for use in DIABLO .........94

3.4.3. Comparison of 1D Vertical Soil Column Response - CARES Using Equivalent Linear Properties versus DIABLO using Ramberg-Osgood Model ...............................................................................97

3.4.4. Development of advanced soil models with traceability to EPRI test data....................... 100

3.5. Time-domain SSI analysis of notional SMR Reactor and Comparison to SASSI results ............107

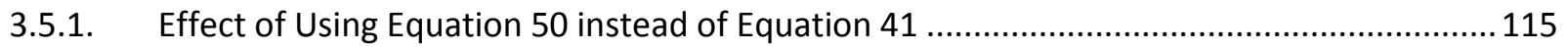

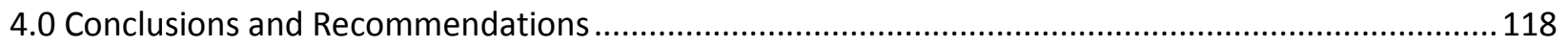

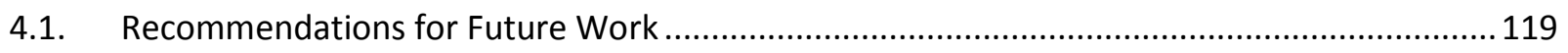

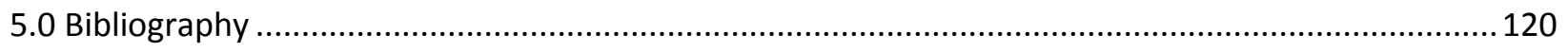

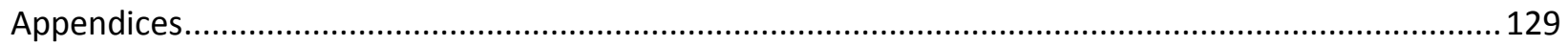




\section{Table of Figures}

Figure 1 - Bielak Method

Figure 2 - Varying impedance required across the intermediate layer for non-reflection of scattered waves

Figure 3 - Notional Small Modular Reactor. Left - with outer walls removed. Right - with outer walls and piers removed

Figure 4 - SMR embedded in soil model, jagged cut at approximately the $\mathrm{Y}=0$ plane. The $\mathrm{Z}=0$ plane corresponds to the top ground surface.

Figure 5 - Soil Response from CARES (green) and using RAMBO properties (red) for a $0.2 \mathrm{~g}$ earthquake, at a distance $190 \mathrm{ft}$ (left) and $205 \mathrm{ft}$ (right) from the top surface 16

Figure 6 - Peak horizontal accelerations at the structure centerline and edge, for three earthquake motions (red is $0.9 \mathrm{~g}$, blue is $0.5 \mathrm{~g}$, and green is $0.2 \mathrm{~g}$ ), calculated by DIABLO (solid bars) and SASSI (open bars).....

Figure 7 - Peak vertical accelerations at the structure centerline and edge, for three earthquake motions (red is $0.9 \mathrm{~g}$, blue is $0.5 \mathrm{~g}$, and green is $0.2 \mathrm{~g}$ ), calculated by DIABLO (solid bars) and SASSI (open bars) .... 18 Figure 8 - Comparison of SASSI (black) and DIABLO (red) at the center of the basemat ...........................20 Figure 9 - Comparison of SASSI (black) and DIABLO (red) for Roof Edge............................................... 22 Figure 10 - Comparison of SASSI (black) and DIABLO (red) at the structure edge, on the first floor ..........22 Figure 11 - Comparison of DIABLO with impedance matching (red) and without (blue) for the structure first floor edge. .24

Figure 12 - Reactor and Containment Structure, Sheet 1, Elevation View .............................................37

Figure 13 - Notional Reactor Model, Sheet 2, Section View Downward towards Base Mat ......................38

Figure 14 - Reactor and Containment Structure, Sheet 3, Section View Downward towards Floor 1 ........ 39

Figure 15 - Reactor and Containment Structure, Sheet 4, Section View Downward towards Floor 2 ....... 40

Figure 16 - Reactor and Containment Structure, Sheet 5, Section View Downward toward Floor 3......... 41

Figure 17 - Structural Elements - Material and Cross-Sectional Properties............................................43

Figure 18 - Beam and Stick Elements Material and Cross-Sectional Properties...................................... 43

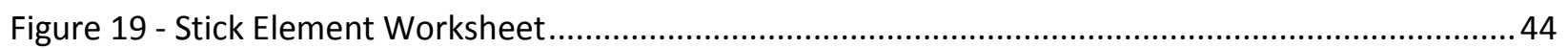

Figure 20 - Notes related to Material and Sectional Properties ..............................................................4 44

Figure 21 - Vertical Mesh Density. The dimensions on the left are the size of each element. The top of the graphic is the centerline of the roof, the bottom of the graphic is the centerline of the basemat, and the zero surface is shown as "ground level" on Figure 12 ..................................................................45

Figure 22 - Reactor structure with outer walls and upper and lower piers removed for clarity................46

Figure 23 - Reactor structure with outer walls removed for clarity .......................................................46

Figure 24 - Soil Layers, EPRI-93 Shear Modulus Degradation, Illustrated .............................................. 50

Figure 25 - Soil Layers, EPRI-93 Hysteretic Damping, Illustrated ..........................................................51

Figure 26 - Reactor model embedded in soil, with a jagged cut at approximately the $Y=0$ plane. The $Z=0$ plane corresponds to the top ground surface. 52 Figure 27 - Calculation of equivalent-linear soil properties using SHAKE (or CARES) assuming a verticallypropagating shear wave corresponding to a particular earthquake..... .58 
Figure 28 - Hysteresis and Modulus Degradation as a result of cyclic effects in liquefiable sands (Matasovic, 1993) 59

Figure 29- Shear Modulus Degradation and Damping curves as a function of confining pressure (Zhang 2005)

Figure 30 - LS-DYNA results using Rayleigh damping versus SASSI results (Xu, 2006) 64

Figure 31 - Comparison of normalized equivalent static stiffness (left) and loss factor (right) for various models, from (Balmes, 2006)..... .66

Figure 32 - Comparison of frequency-independent hysteresis loops (a) versus typical experimental results (b) - taken from Figure 3 of (Bert, 1973). .67

Figure 33 - Dahl model hysteresis loop - taken from Figure 13 of (Padthe, 2008) .................................68

Figure 34 - Typical hysteresis curves with definition of damping .......................................................70 Figure 35 - Finite element mesh for LS-DYNA analysis - taken from Figure 4.1.2-3 of (Xu, et. al., 2006).. 72 Figure 36 - Zoomed in finite element mesh for LS-DYNA analysis - taken from Figure 4.1.2-4 of (Xu, et. al., 2006) .73

Figure 37 - Rock Outcrop Motions versus Layered Half space - taken from Figure 3 of (Schnabel, 1972) 74 Figure 38 - Transfer functions for rock outcrop versus rock base - taken from Figure 4 of (Schnabel, 1972) .75

Figure 39 - Performance of various simple absorbing boundary conditions - taken from Figure 6 of (Kausel, 1981).... .76

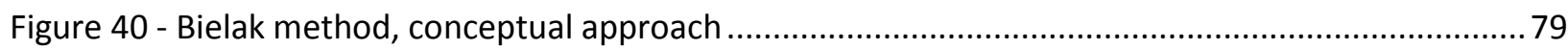

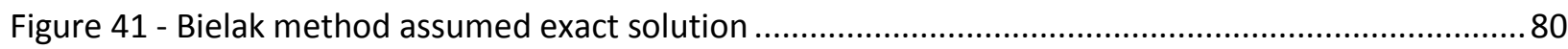

Figure 41 - Bielak method showing the intermediate region ............................................................. 81

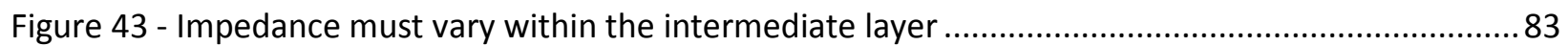

Figure 44 - Perfectly Matched Layer as a discretized, truncated perfectly matched medium (Savadatti,

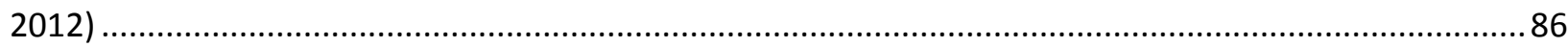

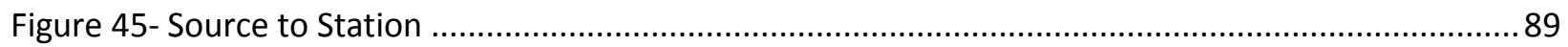

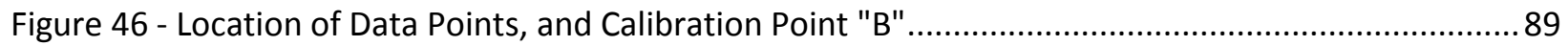

Figure 47 - Synthetic soil motions by Hutchings compared with Costantino data (Hutchings, 2012) .......90

Figure 48 - Comparison of $X$ component acceleration spectra Hutchings Data versus Costantino data

(Hutchings, 2012) at Point B ............................................................................................................ 91

Figure 49 - CARES-derived iterated modulus and damping for $0.2 \mathrm{~g}$ case ............................................ 93

Figure 50 - CARES-derived iterated modulus and damping for $0.5 \mathrm{~g}$ case ............................................. 93

Figure 51 - CARES-derived iterated modulus and damping for the $0.9 \mathrm{~g}$ case ..........................................94

Figure 52 - Result of Ramberg-Osgood Fit using RAMBO to EPRI data for the 9 Soil Layers..................... 94

Figure 53 - Graphical Representation of Ramberg-Osgood Fits to Soil Layers 1-4 ..................................95

Figure 54 - Graphical Representation of Ramberg-Osgood Fits to Soil Layers 5-8 ..................................96

Figure 55 - Graphical Representation of Ramberg-Osgood Fits for Soil Layer 9 .....................................96

Figure 56 -1D Soil Column, 0.2g earthquake, 5\% Response Spectra: DIABLO w/o viscous damping (red) versus CARES (green), distance 0 and $30 \mathrm{ft}$ from top surface

Figure 57 - 1D Soil Column, 0.2g earthquake, 5\% Response Spectra: DIABLO w/o viscous damping (red) versus CARES (green) 1-d with no viscous damping, distance 252 and 278 feet from top surface....... 
Figure 58 - 1D Soil column, 0.2g earthquake, 5\% Response Spectra: DIABLO w/o viscous damping(red) versus CARES 1-d distance 303 and $370 \mathrm{ft}$ from top surface.

Figure 59 - 1D Soil column, 0.2g earthquake, 5\% Response Spectra: DIABLO with viscous damping(red) versus CARES $1-\mathrm{d}$ distance 0 and $30 \mathrm{ft}$ from top surface.

Figure 60 - 1D Soil column, 0.2g earthquake, 5\% Response Spectra: DIABLO with viscous damping(red) versus CARES 1-d distance 190 and $205 \mathrm{ft}$ from top surface.

Figure 61 - 1D Soil column, 0.2g earthquake, 5\% Response Spectra: DIABLO with viscous damping(red) versus CARES 1-d distance 303 and $370 \mathrm{ft}$ from top surface.

Figure 62 - Effective of varying the undrained strength for the Von Mises/Perfectly Plastic Model on the modulus degradation and damping ratios, assuming $2 \%$ viscous damping - see Figure 2.11 of (Pisano, et. al., 2012) 101

Figure 63 - Calibration 3 of the DPKH model versus typical shear modulus degradation and damping curves [Seed and Idris] - see Figure 2.19 of (Pisano, et. al., 2012) . 103 Figure 64 - Shear Modulus Degradation and Damping Curves - Comparison to Experimental Data, using Equation 3.33 of (Pisano, et. all, 2012) - see Figure 3.6 of the aforementioned work. 105 Figure 65 - Calibration of the Pisano-Jeremic model with some manual tuning - see Figure 3.7 of (Pisano, et. al., 2012) 105

Figure 66 - Effect of varying confining pressure on the Pisano-Jeremic model, see Figure 3.8 of (Pisano, et. al., 2012) 106 Figure 67 - Effective of different dilatancy characteristics on the response of the soil - see Figure 3.12 of (Pisano, et. al., 2012) 106 Figure 68 - Location of Comparison Points between SASSI and DIABLO .......................................... 108 Figure 69 - Peak horizontal accelerations at the structure centerline and edge, for three earthquake motions, calculated by DIABLO and SASSI...

Figure 70 - Peak vertical accelerations at the structure centerline and edge, for three earthquake motions, calculated by DIABLO and SASSI

Figure 71 - Comparison of SASSI (black) and DIABLO (red) at the center of the basemat ......................112

Figure 72 - Comparison of SASSI (black) and DIABLO (red) at the Roof Edge......................................... 113

Figure 73 - Comparison of SASSI (black) and DIABLO (red) for the $1^{\text {st }}$ Floor Center................................ 114

Figure 74 - Comparison of Peak Acceleration values, SASSI (open) versus DIABLO WITHOUT Impedance matching (filled).

Figure 75 - Comparison of DIABLO with impedance matching (red) and without (blue), 1st Floor edge $\mathrm{X}=14$

Figure 76 - 1D Soil column, 0.2g earthquake, 5\% Response Spectra: DIABLO w/o viscous damping(red) versus CARES (green), distance 0 and $30 \mathrm{ft}$ from top surface 129 Figure 77 - 1D Soil column, 0.2g earthquake, 5\% Response Spectra: DIABLO w/o viscous damping(red) versus CARES (green), distance 52 and $123 \mathrm{ft}$ from top surface 129 Figure 78 - 1D Soil column, 0.2g earthquake, 5\% Response Spectra: DIABLO w/o viscous damping(red) versus CARES (green), distance 190 and $205 \mathrm{ft}$ from top surface 129 Figure 79 - 1D Soil column, 0.2g earthquake, 5\% Response Spectra: DIABLO w/o viscous damping(red) versus CARES (green), distance 252 and $278 \mathrm{ft}$ from top surface 130 
Figure 80 - 1D Soil column, 0.2g earthquake, 5\% Response Spectra: DIABLO w/o viscous damping(red) versus CARES (green), distance 303 and $370 \mathrm{ft}$ from top surface 130

Figure 81 - 1D Soil column, 0.2g earthquake, 5\% Response Spectra: DIABLO with viscous damping(red) versus CARES (green), distance 0 and $30 \mathrm{ft}$ from top surface

Figure 82 - 1D Soil column, 0.2g earthquake, 5\% Response Spectra: DIABLO with viscous damping(red) versus CARES (green), distance 52 and $123 \mathrm{ft}$ from top surface

Figure 83 - 1D Soil column, 0.2g earthquake, 5\% Response Spectra: DIABLO with viscous damping(red) versus CARES (green), distance 190 and $205 \mathrm{ft}$ from top surface

Figure 84 - 1D Soil column, 0.2g earthquake, 5\% Response Spectra: DIABLO with viscous damping(red) versus CARES (green), distance 252 and $278 \mathrm{ft}$ from top surface

Figure 85 - 1D Soil column, 0.2g earthquake, 5\% Response Spectra: DIABLO with viscous damping(red) versus CARES (green), distance 303 and $370 \mathrm{ft}$ from top surface

Figure 86 - 1D Soil column, 0.5g earthquake, 5\% Response Spectra: DIABLO with viscous damping(red) versus CARES (green), distance 0 and $30 \mathrm{ft}$ from top surface

Figure 87 - 1D Soil column, 0.5g earthquake, 5\% Response Spectra: DIABLO with viscous damping(red) versus CARES (green), distance 52 and $123 \mathrm{ft}$ from top surface

Figure 88 - 1D Soil column, 0.5g earthquake, 5\% Response Spectra: DIABLO with viscous damping(red) versus CARES (green), distance 190 and $205 \mathrm{ft}$ from top surface

Figure 89 - 1D Soil column, 0.5g earthquake, 5\% Response Spectra: DIABLO with viscous damping(red) versus CARES (green), distance 252 and $278 \mathrm{ft}$ from top surface

Figure 90 - 1D Soil column, 0.5g earthquake, 5\% Response Spectra: DIABLO with viscous damping(red) versus CARES (green), distance 303 and $370 \mathrm{ft}$ from top surface 134 Figure 91 - 1D Soil column, 0.9g earthquake, 5\% Response Spectra: DIABLO with viscous damping(red) versus CARES (green), distance 0 and $30 \mathrm{ft}$ from top surface 135 Figure 92 - 1D Soil column, 0.9g earthquake, 5\% Response Spectra: DIABLO with viscous damping(red) versus CARES (green), distance 052 and $123 \mathrm{ft}$ from top surface 135 Figure 93 - 1D Soil column, 0.9g earthquake, 5\% Response Spectra: DIABLO with viscous damping(red) versus CARES (green), distance 190 and $205 \mathrm{ft}$ from top surface 135 Figure 94 - 1D Soil column, 0.9g earthquake, 5\% Response Spectra: DIABLO with viscous damping(red) versus CARES (green), distance 252 and $278 \mathrm{ft}$ from top surface 136 Figure 95 - 1D Soil column, 0.9g earthquake, 5\% Response Spectra: DIABLO with viscous damping(red) versus CARES (green), distance 303 and $370 \mathrm{ft}$ from top surface 136 Figure 96 - Response Spectra Comparison DIABLO (red) versus SASSI (black), Basemat Center ..............137 Figure 97 - Response Spectra Comparison, DIABLO (red) versus SASSI (black), First Floor Center.......... 138 Figure 98 - Response Spectra Comparison, DIABLO (red) versus SASSI (black), 2nd Floor Center........... 139 Figure 99 - Response Spectra Comparison, DIABLO (red) versus SASSI (black), Reactor Top Cover .........140 Figure 100 - Response Spectra Comparison, DIABLO (red) versus SASSI (black), 3rd Floor Center ..........141 Figure 101 - Response Spectra Comparison, DIABLO (red) versus SASSI (black), Roof Center ................ 142 Figure 102 - Response Spectra Comparison, DIABLO (red) versus SASSI (black), BaseMat X=14 Edge.... 143 Figure 103 - Response Spectra Comparison, DIABLO (red) versus SASSI (black), First Floor X=14 Edge.. 144 Figure 104 - Response Spectra Comparison, DIABLO (red) versus SASSI (black), 2nd Floor X=14 Edge... 145 Figure 105 - Response Spectra Comparison, DIABLO (red) versus SASSI (black), 3rd Floor X=14 Edge ... 146 
Figure 106 - Response Spectra Comparison, DIABLO (red) versus SASSI (black), Roof X=14 Edge 147

Figure 107 - Response Spectra Comparison, DIABLO (red) versus SASSI (black), BaseMat Y=14 Edge ....148

Figure 108 - Response Spectra Comparison, DIABLO (red) versus SASSI (black), 1st Floor Y=14 Edge .... 149

Figure 109 - Response Spectra Comparison, DIABLO (red) versus SASSI (black), 2nd Floor Y=14 Edge... 150

Figure 110 - Response Spectra Comparison, DIABLO (red) versus SASSI (black), 3rd Floor Y=14 Edge ... 151

Figure 111 - Response Spectra Comparison, DIABLO (red) versus SASSI (black), Roof Y=14 Edge...........152 


\subsection{Executive Summary}

For over 30 years, modeling of nuclear power reactors for seismic safety has been conducted using a simplified soil-structure interaction (SSI) analysis approach that has proven remarkably powerful given the then available knowledge of the response of structures, soils, and their interactions under the cyclical loading of seismic shaking [1]. Various modifications and adjustments to this modeling framework have been made to approximate seismic response in light of new insights into the SSI phenomenon. However, many of the inherent limitations of the overall approach remain.

Technology related to earthquake rupture, seismic wave propagation, structural mechanics, and soil models have all advanced enormously since the early eighties, grounded substantially on the rapid advance in computing power. Much more robust models are now available to address each part of power reactor seismic response (source to site) and to address all of the relevant physics including nonlinear features of site and structure response. Given recent events in which nuclear power reactors have been subjected to beyond-design seismic shaking, as well as the emergence of advanced reactor designs, an important and urgent question is whether a coherent new approach incorporating advanced nonlinear models and modeling techniques using high performance computing can predict nuclear reactor response to earthquakes with higher fidelity than standard techniques in current use. This report takes a first quantitative step toward a clear answer.

At the start of the current study, a panel of earthquake engineering experts was convened by DOE's Nuclear Energy Advanced Modeling and Systems (NEAMS) Campaign [2] to review soil-structure interaction (SSI) analysis modeling capabilities using current state-of-practice frequency-domain approaches and modern, emerging time-domain computational mechanics approaches. The current frequency-domain approach uses the SASSI program [1], [3]. One objective of the expert panel was to identify inadequacies in frequency-domain approaches that could be addressed by modern time-domain SSI analysis methods. Based on the recommendations of these experts, a team of five DOE laboratories investigated the feasibility of developing a Generalized Methodology for Soil-Structure Interaction (SSI) using Nonlinear Time Domain analysis. Lawrence Livermore National Laboratory (LLNL) led the multi-lab team that included Argonne National Laboratory (ANL), Brookhaven National Laboratory (BNL), Lawrence Berkeley National Laboratory (LBNL), and Oak Ridge National Laboratory (ORNL). The investigation is a part of the NEAMS Campaign in the US DOE Office of Nuclear Energy (NE-41). Even though the methodology developed would be applicable to any soil-structure interaction problem, it is particularly applicable to a large, heavy building with significant embedment depth, where the possibility for SSI effects is high. Such a configuration is typically envisioned for Small Modular Reactors (SMRs).

A generalized time-domain method for Soil-Structure Interaction is developed, based upon an extension of the Bielak Method. Using a simple hysteretic model based on the 1-D Ramberg-Osgood formulation, the study developed a methodology that can produce results with excellent comparability with SASSI. Because of the use of a general nonlinear time-domain formulation, the methodology provides a path forward for investigation of other sources of nonlinearity, including those associated with more physically-realistic material models incorporating pore-pressure effects, gap opening/closing, the effect of nonlinear structural elements, and 3D seismic inputs.

To support consideration of the time domain analysis method for licensing, this report benchmarks the new method to current state-of-practice SASSI analysis results. The experts agreed that the benchmark 
should use a relatively simple earthquake motion, a soil that could be reasonably represented in both SASSI and time-domain models, a method of preventing spurious reflections from computational boundaries, a reactor building structure that could interact with the soil, and measurement locations suitable to elicit the resulting motions at a number of locations within the structure. Furthermore, the benchmark should provide for future investigation into additional sources of variance, such as 3D input wave motion, nonlinear geometric effects (e.g., gap opening/closing), and nonlinear structural elements.

This report primarily documents the development of LLNL's nonlinear time-domain SSI analysis technique, and compares the responses predicted by this method with those by the SASSI-based method performed by BNL. The comparison demonstrates that for this relatively simple benchmark problem, the nonlinear time-domain method produces similar results to those produced by the SASSIbased method. Since this nonlinear time-domain method is generally applicable or easily adaptable to various complex dynamic soil and building characteristics (e.g. gap formation and nonlinear material properties) that cannot be simulated in a SASSI-based SSI analysis method, the time-domain method can be used in the future to investigate a series of nonlinearities that are being either ignored or indirectly considered in the current frequency-domain SASSI-based method. In addition, because the timedomain method takes advantage of modern computational techniques, it can use modern highperformance parallel computing architectures, thereby greatly improving analysis throughput.

\subsection{The Need for a Generalized Nonlinear Time-Domain SSI Analysis Methodology}

The current state of practice, as represented by the computer code SASSI, solves the equations of motion in the frequency-domain. Such an approach is inherently limited to linear representation of soil properties as it relies on the principle of superposition. Soils are strongly nonlinear even at small strains, featuring both strain-dependent stiffness and strain-dependent damping characteristics, as documented for example in the EPRI report on strain-dependent soil characteristics [4]. SASSI can incorporate these strain dependencies only indirectly, via the "equivalent-linear" method. In this method the soil stiffness and damping characteristics are selected for each soil layer based upon a prediction developed using a the particular earthquake time history under consideration and a one-dimensional soil column simulator such as SHAKE [5]. Soils typically exhibit stiffness and damping properties that are significantly affected by changes in three-dimensional confining pressures as the seismic event progresses [6]. Many soils typically exhibit significant evolution of the soil response over a number of strain cycles, often in concert with pore water pressure and content evolution [7]. The current SASSI approach is not capable of incorporating these latter two effects even indirectly.

In the "equivalent-linear" method used by SASSI, the maximum strains as a function of vertical position in the soil column are estimated for a specified seismic input via a 1D soil column solution (without the structure), and constant stiffness and damping values are selected for each soil layer in the 3-D analysis based upon this prediction. This method may be non-conservative in a number of cases:

- It may underpredict the response in regions with low (but significant) amplitude response, as often occurs at higher frequencies [8]

- It may underpredict deformation at locations of local stress concentrations, where softening in excess of that predicted may occur [9] 
- It cannot account for the pressure dependence of soils [10], an effect that is particularly important in the presence of "raft uplift" that may occur under portions of the basemat during rocking motions [11].

- The "hysteretic damping" used in equivalent-linear analyses, although perhaps better able to model certain experimental results than simple Kelvin-Vogt models typically used in linear timedomain analysis is mathematically suspect since it cannot be transformed to a causal function in the time-domain [12].

- Current frequency-domain codes suffer from long analysis time for large models. This has led to the adoption of algorithmic modifications ("SASSI Subtraction Method") that improve analysis turnaround time, but have been shown [13] to produce results that may contain non-physical peaks in the response, especially at high frequencies.

- Equivalent-linear analyses cannot account for geometric nonlinearity resulting from separation (contact/release) at the interface between the soil and the structure during a seismic event/

\subsection{Review of Current Nonlinear Time-Domain SSI Analysis Methods}

The current effort to develop a generalized nonlinear time-domain SSI analysis methodology is intended to overcome the shortcomings of the frequency-domain SASSI approach, and to improve on the existing (but infrequently-used) nonlinear time-domain SSI analysis methods. First, a critical review of existing literature on time-domain SSI analysis methods was performed that revealed:

- Time-domain analysis is capable of routinely modeling fully nonlinear soils with straindependent soil stiffness properties.

- Time-domain analysis can account for geometric nonlinearity or gaps resulting from separation at the interface of the soil and structure.

- Because of the granular, frictional nature of soils, accurate models of soil response require nonlinear behavior even at near-zero strains [14].

- In recent years, nonlinear models have been developed which can adequately model the hysteretic behavior of soils in the time domain. In advanced versions, such features as pressure dependence and cyclic degradation are also included [15].

- Major remaining impediments to the use of time-domain analysis include the related problems of proper input of seismic motions and the absorption of scattered waves, both areas of continued research. As a result, many time-domain analyses suffered from issues of reliability due to imperfect boundary conditions, or the need for large and unwieldy models to minimize effects due to imperfect boundary conditions.

\subsection{Development of LLNL's Nonlinear Time-Domain SSI Analysis Methodology}

To develop a state-of-the-art nonlinear time-domain SSI analysis method, LLNL started with, and extended, a method developed by Bielak and coworkers [16] [17]. The Bielak method (see Figure 1) provides a rational means for applying seismic input motion. The method consists of two primary steps:

- Partition the soil around the building into three regions, $\Omega_{1}$ (the inner region immediately around the building), $\Omega_{\| \mid}$(the outer region that prevents wave reflection), and $\Omega_{\|||}$(the intermediate region), as shown in Figure 1. 
- Drive the inner region $\Omega_{1}$, according to a prescribed seismic input time history, through forces applied in the intermediate region $\Omega_{\| 11}$. Assuming the seismic input time history is consistent with the discrete solution in $\Omega_{\mid}$(absent the structure), the motion in the outer region $\Omega_{\|}$consists only of the waves scattered by the presence of the structure.

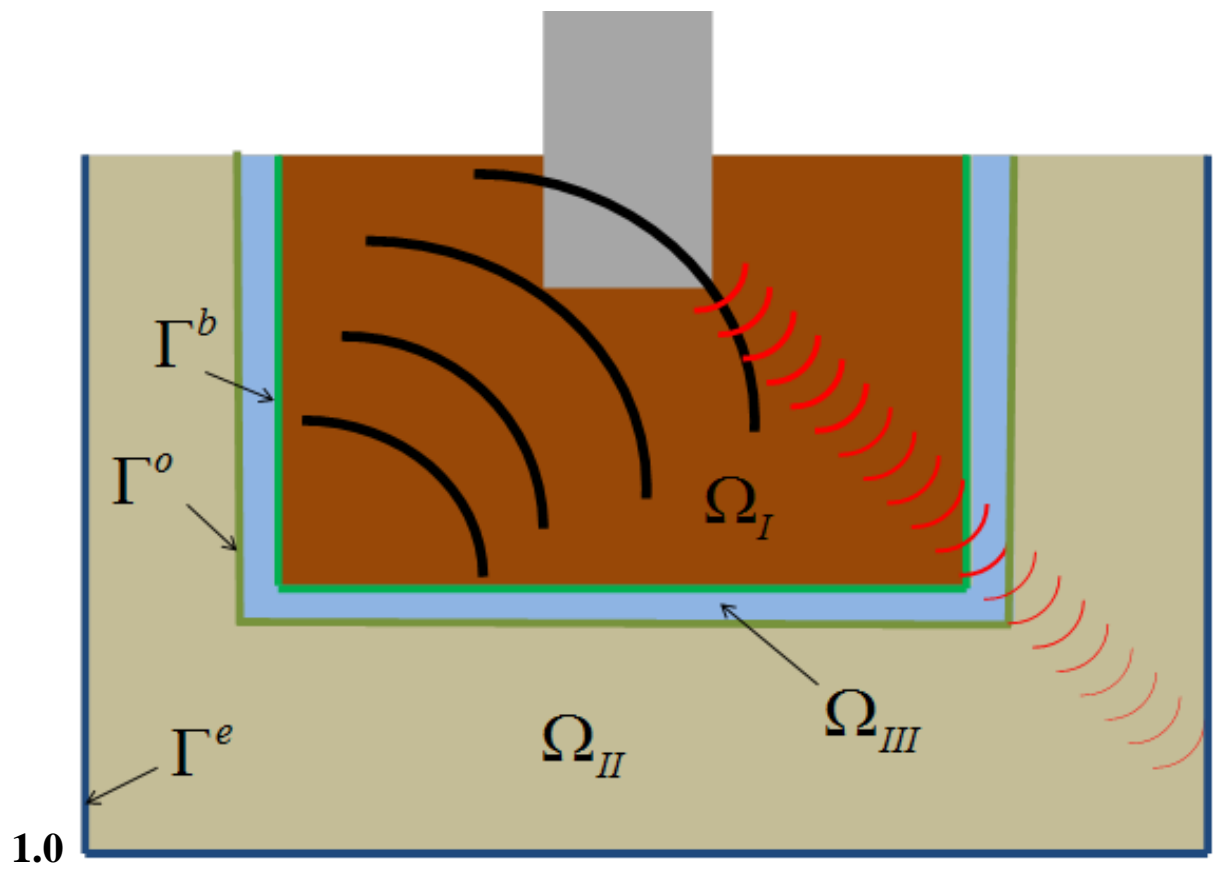

Figure 1 - Bielak Method

The Bielak method has a number of benefits. Chief among them is the fact that the scattered waves in region $\Omega_{\|}$have both lower magnitude and higher geometric attenuation (since they can be approximated by a point source) in comparison with the original seismic input. Because of the decreased magnitude of the motion within $\Omega_{\| 1}$, the magnitude of the motion reaching the outer boundary has also been decreased significantly. Hence, the absorbing boundary condition applied to the external boundary, $\Gamma_{\mathrm{e}}$, does not have to work as hard. Thus, simple but not $100 \%$ effective absorbing boundary conditions can be used without adversely affecting the solution within the inner region, $\Omega_{1}$.

In the original Bielak method, the intermediate region $\Omega_{\text {III }}$ was formulated as a linear visco-elastic material, and the region was assumed to be a single layer of elements (one element thick). However, adequate capture of strain-dependent damping and moduli of soils requires nonlinear soil behavior down to zero strain. Given this situation, the following is evident:

- A Bielak-style method that can drive materials that have non-linear behavior even at zero strain is desirable.

- Such a method should allow for unimpeded passage of scattered waves through regions $\Omega_{\text {III }}$ and $\Omega_{\| \mid}$. This requires the impedance of the material within the intermediate region $\left(\Omega_{\| 11}\right)$ to vary smoothly between the inner region $\left(\Omega_{1}\right)$ and the outer region $\left(\Omega_{\| 1}\right)$, see Figure 2 . 


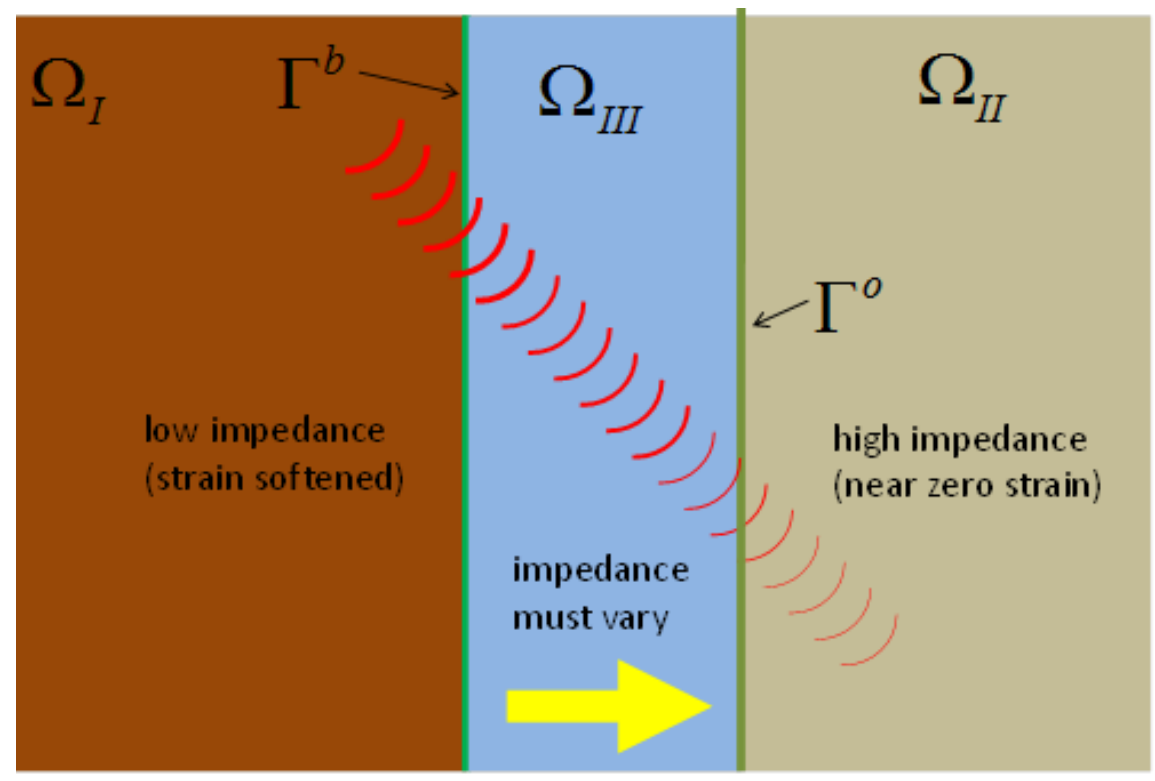

Figure 2 - Varying impedance required across the intermediate layer for non-reflection of scattered waves

LLNL developed a modified Bielak method that:

- Is valid for arbitrary non-linear materials.

- Accommodates intermediate regions of arbitrary thickness (more than one element thick).

- Generates nominal stresses and strains (and thus the resulting impedances) in the intermediate region $\left(\Omega_{\| 1}\right)$ varying smoothly between the inner region $\left(\Omega_{1}\right)$ and the outer region $\left(\Omega_{\| 1}\right)$, as in Figure 2.

The LLNL-developed modified Bielak method has been implemented in LLNL's nonlinear time-domain structural mechanics code DIABLO. The effectiveness of the technique was demonstrated in three benchmark calculations by applying the method to a finite element SSI model representing a typical small modular reactor building that is partially buried in soil (see Figures 3 and 4). This SSI model was subjected to seismic motion consisting of a vertically propagating shear wave consistent with three nominal earthquake levels, $0.2 \mathrm{~g}, 0.5 \mathrm{~g}$, and $0.9 \mathrm{~g}$.

For comparison with the DIABLO analysis, the same earthquakes were modeled using SASSI, which uses the "equivalent linear method", whereby each soil layer has constant soil stiffness and damping parameters that are selected for the earthquake level. These soil model parameters were determined using an iterative method via the computer code CARES [18], [19]. Existing measured soil data ${ }^{1}$ from a 9-layer soil column at a DOE site were used in combination with CARES simulations to predict the soil behavior for each of three notional earthquakes with nominal acceleration levels of $0.2 g, 0.5 \mathrm{~g}$, and $0.9 \mathrm{~g}$. In the "equivalent-linear" approach, there are three different pairs of material properties (modulus and damping) assigned to each soil layer, one for each of the earthquake levels. That is, the SASSI approach uses a soil model that is earthquake-dependent, but is not directly dependent on strains that develop during the seismic event.

\footnotetext{
1Shear modulus degradation ( $\left.G / G_{\max }\right)$ and damping curves, as a function of maximum strain per cycle, per the EPRI protocol [4].
} 

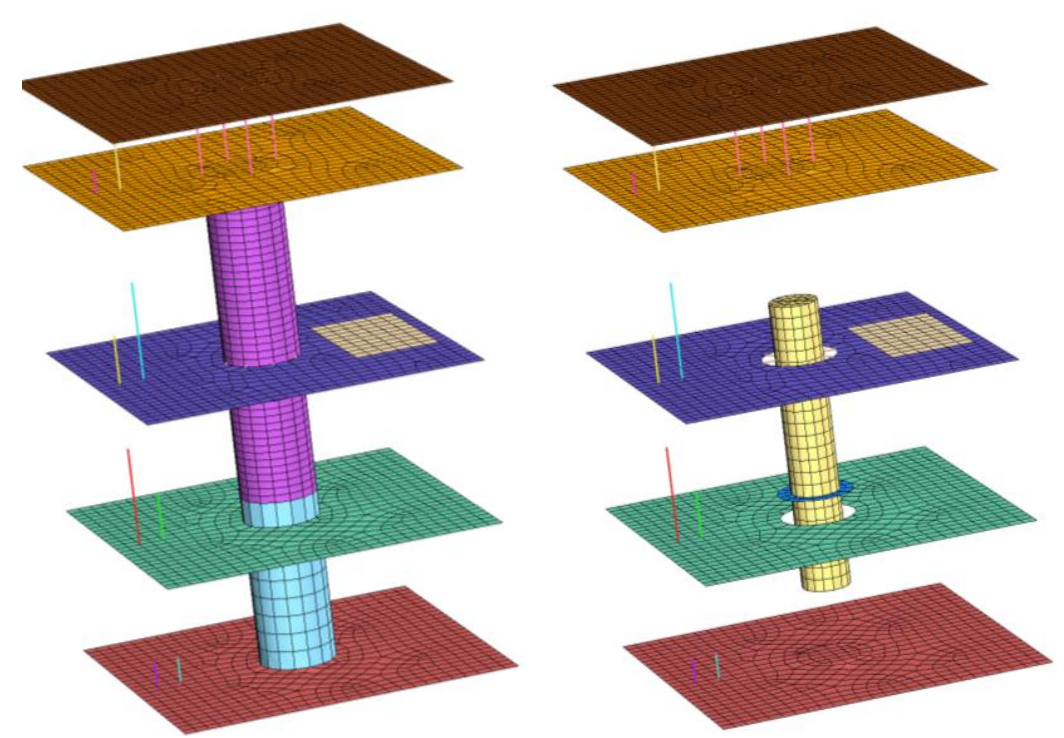

Figure 3 - Notional Small Modular Reactor. Left - with outer walls removed. Right - with outer walls and piers removed

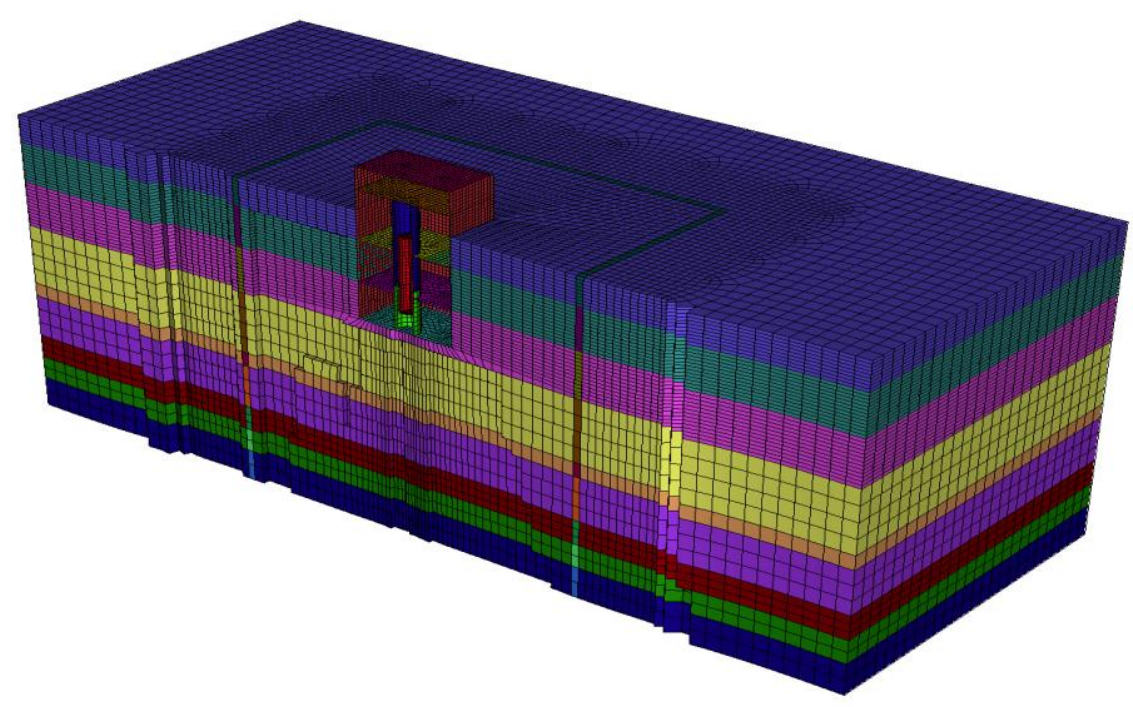

Figure 4 - SMR embedded in soil model, jagged cut at approximately the $Y=0$ plane. The $Z=0$ plane corresponds to the top ground surface.

In DIABLO, the nonlinear hysteretic characteristics of the soil in which the reactor building is partially buried were represented by a Ramberg-Osgood material model [20] that requires the determination of four parameters to fit the behavior of each of the 9 soil layers, using an algorithm (RAMBO) developed earlier at LLNL [21]. The parameters were determined by fitting to the same DOE site data that were used in the CARES analysis for SASSI. In contrast to the "equivalent linear" approach, the parameters were only fit once, since they depend only on the soil data and not on the particular earthquake. That is, the DIABLO approach uses a soil model that is dependent on strains that develop during the seismic event, not on a-priori predictions. 
LLNL's time-domain DIABLO SSI model, including the representation of the reactor building and components, is almost identical in configuration to the SASSI model. The differences in the models were in the representation of soil properties in the two models, and in the use of the extended Bielak method in the DIABLO model to preclude computational wave reflections, these reflections are handled via a different methodology in SASSI based on the frequency-domain solution of Waas [22]. A model view is shown in Figure 4, which has a jagged plane cut through the center of the reactor building to visualize the building internals ${ }^{2}$.

\subsection{SSI Analyses and Results}

The 1-D soil column analyses used in CARES were repeated using a 1-D DIABLO run (with no reactor structure) to benchmark the soil properties developed by the RAMBO code. The benchmarking revealed that the addition of a small amount of viscous dissipation improved the comparison with CARES. Figure 5 shows that the CARES results (green curves) and the DIABLO results (red curves) are similar at two depths in the soil.
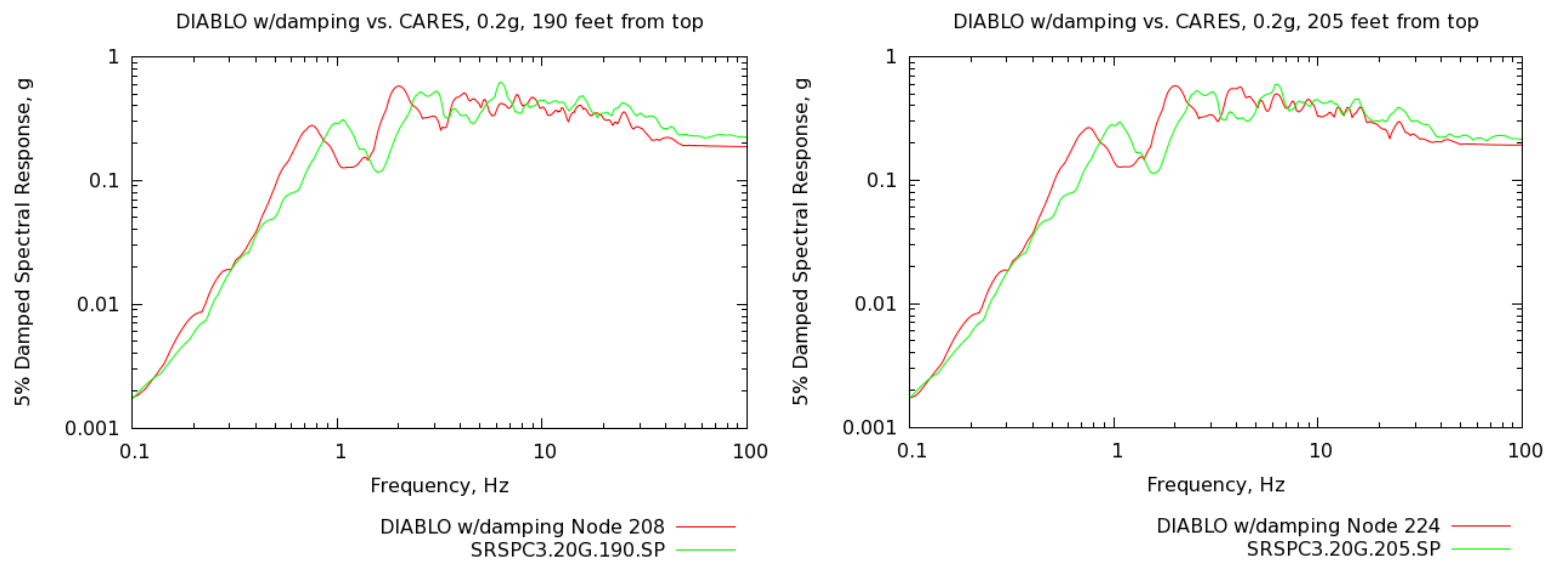

Figure 5 - Soil Response from CARES (green) and using RAMBO properties (red) for a $0.2 \mathrm{~g}$ earthquake, at a distance $190 \mathrm{ft}$ (left) and $205 \mathrm{ft}$ (right) from the top surface

For the SSI analysis of the full structure/soil system, DIABLO simulations were performed for the notional $0.2 \mathrm{~g}, 0.5 \mathrm{~g}$, and $0.9 \mathrm{~g}$ earthquakes and were compared with SASSI simulations performed for the same input earthquakes. Data from the 1-D soil column runs using DIABLO were used as input for the nonlinear Bielak Method. The data were compared using typical acceleration response spectra at various points within the structure. Figures 6 and 7 illustrate the peak horizontal and vertical accelerations, respectively, at the structure centerline and structure edge, at elevations ranging from the base mat to the roof. The input motion was in the " $\mathrm{X}$ " horizontal direction, and the " $\mathrm{Z}$ " direction corresponded to the vertical direction. The analyses were implicit, running on 128 processors.

\footnotetext{
2 For simplicity in meshing, the soil mesh elements sometimes cross the $X=0$ and $Y=0$ planes; thus, the model in Figure 4 shows jagged lines where this is the case.
} 
Approximately 26 seconds of "shake time" required about 12 hours of analysis "wall time".
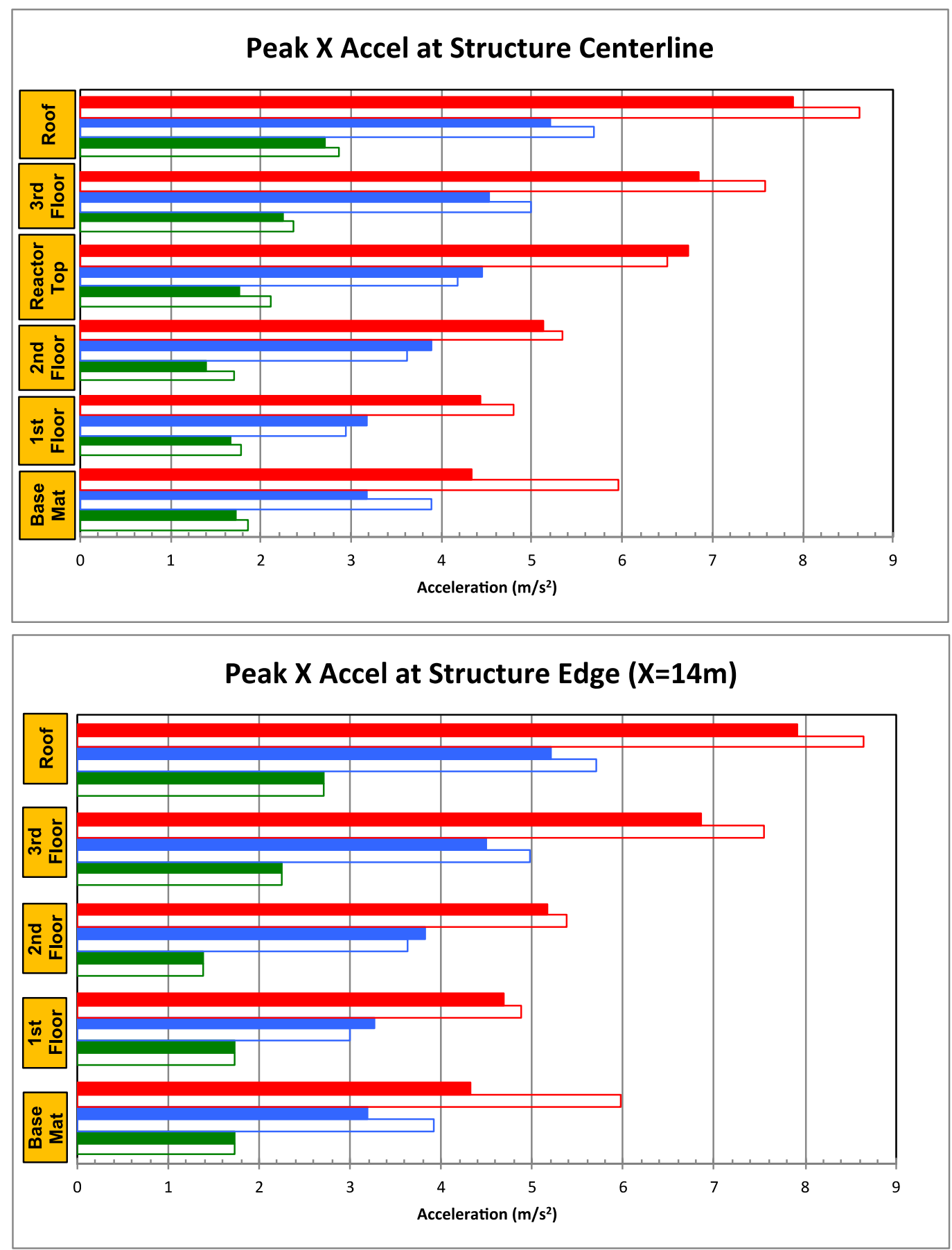

Figure 6 - Peak horizontal accelerations at the structure centerline and edge, for three earthquake motions (red is $0.9 \mathrm{~g}$, blue is $0.5 \mathrm{~g}$, and green is $0.2 \mathrm{~g}$ ), calculated by DIABLO (solid bars) and SASSI (open bars) 

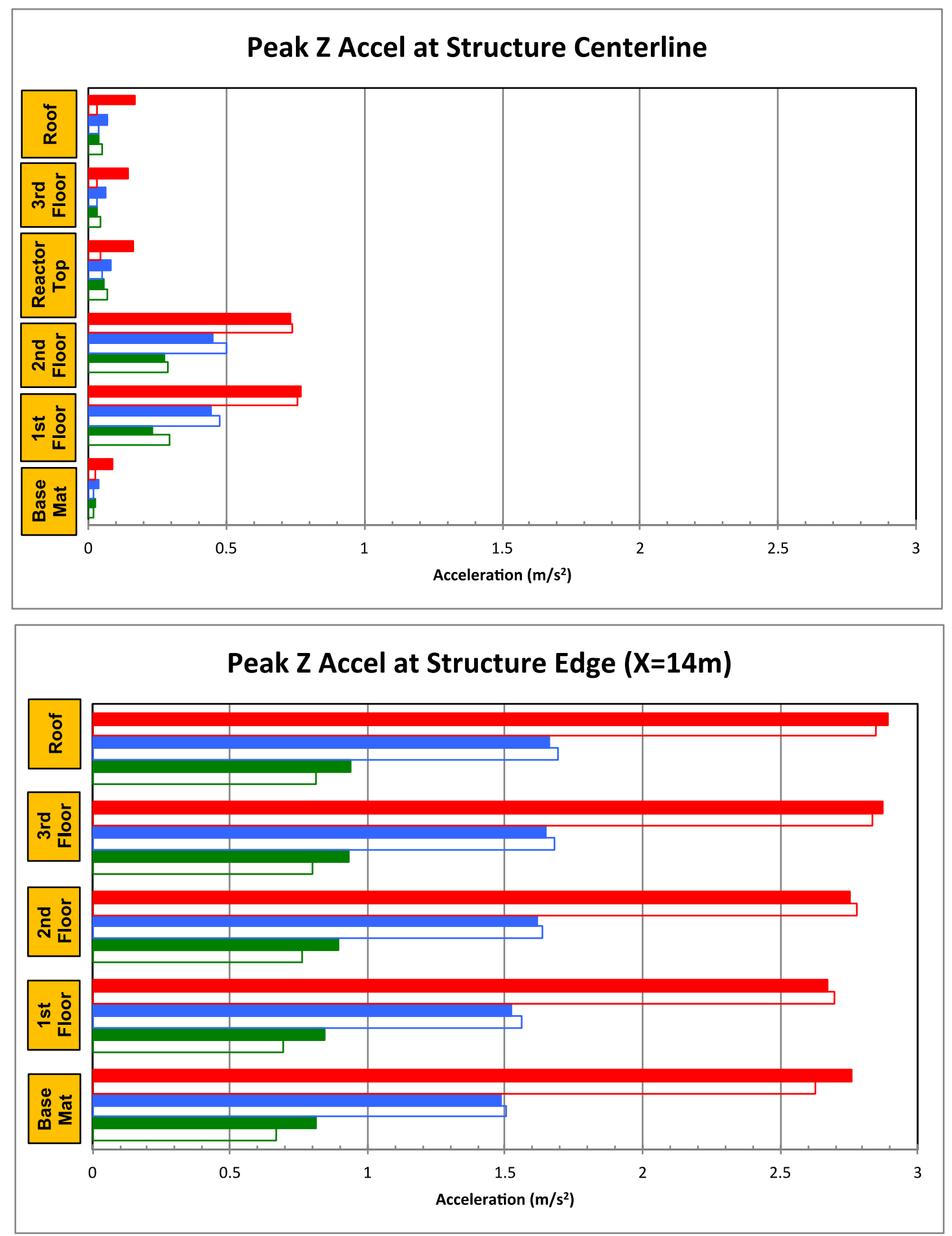

Figure 7 - Peak vertical accelerations at the structure centerline and edge, for three earthquake motions (red is $0.9 \mathrm{~g}$, blue is $0.5 \mathrm{~g}$, and green is $0.2 \mathrm{~g}$ ), calculated by DIABLO (solid bars) and SASSI (open bars)

Inspection of Figure 6, for the horizontal accelerations shows: 
- DIABLO and SASSI are in reasonable agreement for all three earthquake motions, and for all the vertical locations within the structure. Generally (but not universally), SASSI predicts slightly higher maximum accelerations in comparison with DIABLO. This is likely due to the nonlinear nature of DIABLO's material response - in the case of large motions the Ramberg-Osgood material model will strain-soften and increase the damping in comparison with the fixed values (derived from the CARES predictions) used by SASSI. This effect is particularly in evidence for the response at the basemat.

- The horizontal accelerations at each location increase with the intensity of the driving earthquake.

- Horizontal accelerations are generally (but not universally) stronger at the higher locations in the building.

Inspection of Figure 7, for the vertical accelerations shows:

- DIABLO and SASSI are in reasonable agreement at the structure edge for all three earthquake motions, and for all the vertical locations within the structure. The vertical accelerations at each location increase with the intensity of the driving earthquake, and there is not a major variation in vertical acceleration as one moves higher in the building

- At the structure centerline, DIABLO and SASSI are in reasonable agreement at the first and second floor elevations for all three earthquake motions, and for which the vertical motions are considerably higher than at elevations above and below these floors. For DIABLO, the vertical accelerations at each location increase with the intensity of the driving earthquake; but for SASSI, this expected trend is not seen at the upper three levels.

- All the vertical accelerations are small compared to the horizontal accelerations; note that the graphical scale for the vertical accelerations is magnified by a factor of 3 compared to the horizontal accelerations in Figure 6.

- The vertical accelerations at the structure edge are much higher than at the structure centerline, indicating that both models are capturing the rocking motion of the structure within the soil.

Comparisons of response spectra ${ }^{3}$ typical of the obtained results are shown in Figures 8, 9, and 10.

${ }^{3}$ Maximum acceleration magnitude of a harmonic oscillator with $5 \%$ of critical damping to input an acceleration time history, as a function of the oscillator natural frequency - see for example [113]. The acceleration input is the same as was used to generate Figures 6 and 7. 
In Figure 8, the $X$ (horizontal) and $Z$ (vertical) responses are shown at the center of the basemat. The response spectra match well except for the Z-direction component, which is of relatively small magnitude, where the SASSI results are lower than the DIABLO results. For the $X$ component, the peak frequencies are shifted relative to SASSI - most probably a function of the non-linear character of the DIABLO simulation.
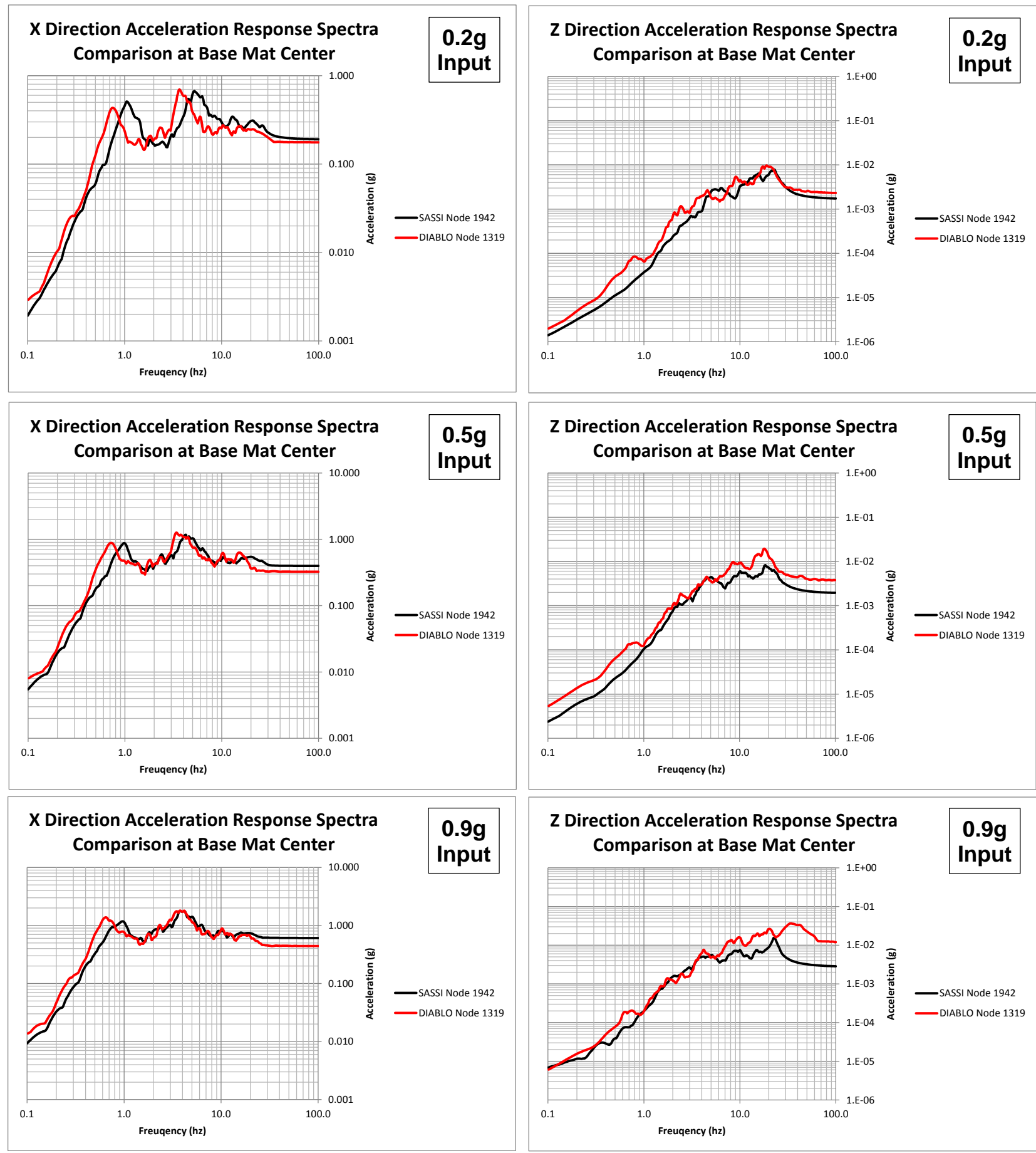

Figure 8 - Comparison of SASSI (black) and DIABLO (red) at the center of the basemat 
In Figure 9, representing the response at the roof edge, both the horizontal and vertical responses match well. The response is large for all cases, due to the significant rocking motions experienced by the reactor, which are felt at this location since it is off-center. Though the general shape of the response matches well, the peak frequencies again are shifted. Frequency shifts are not uncommon when comparing the response of a non-linear model and an equivalent linear approximation.
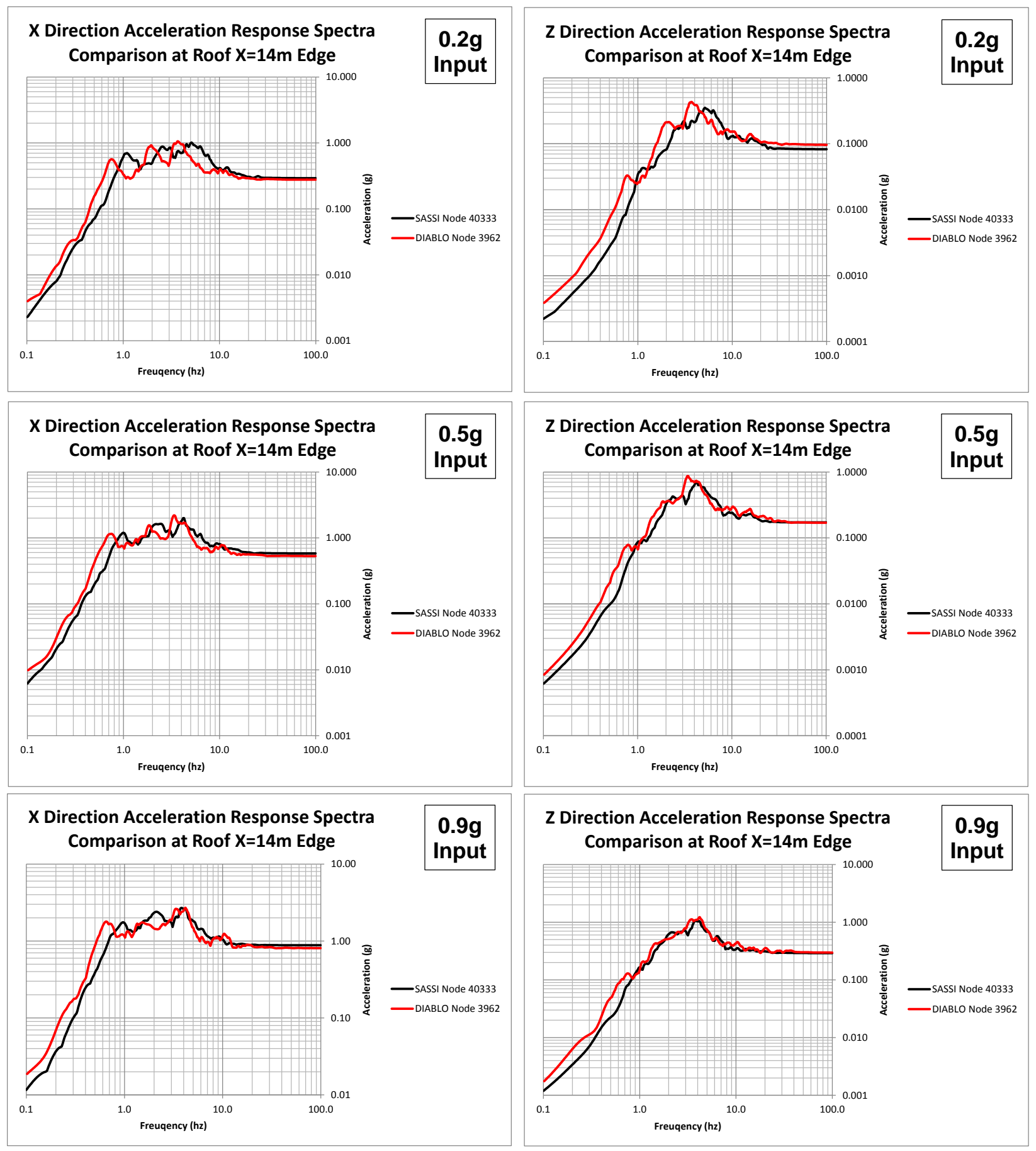


\section{Figure 9 - Comparison of SASSI (black) and DIABLO (red) for Roof Edge}

In Figure 10, the response at the structure edge, on the first floor, generally matches well except at some high frequencies. Again, the peak frequencies are shifted, especially for the $0.2 \mathrm{~g}$ and $0.5 \mathrm{~g}$ cases.
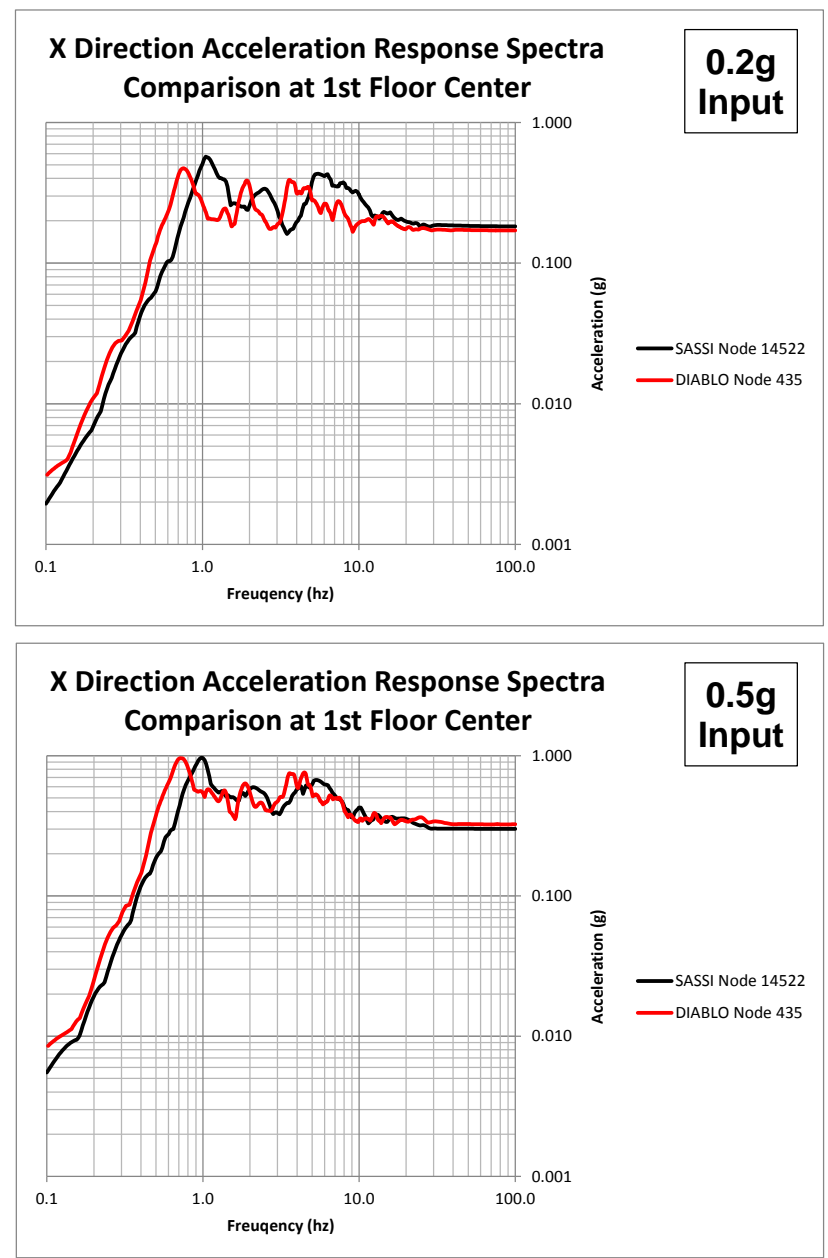

\section{Direction Acceleration Response Spectra} Comparison at 1st Floor Center
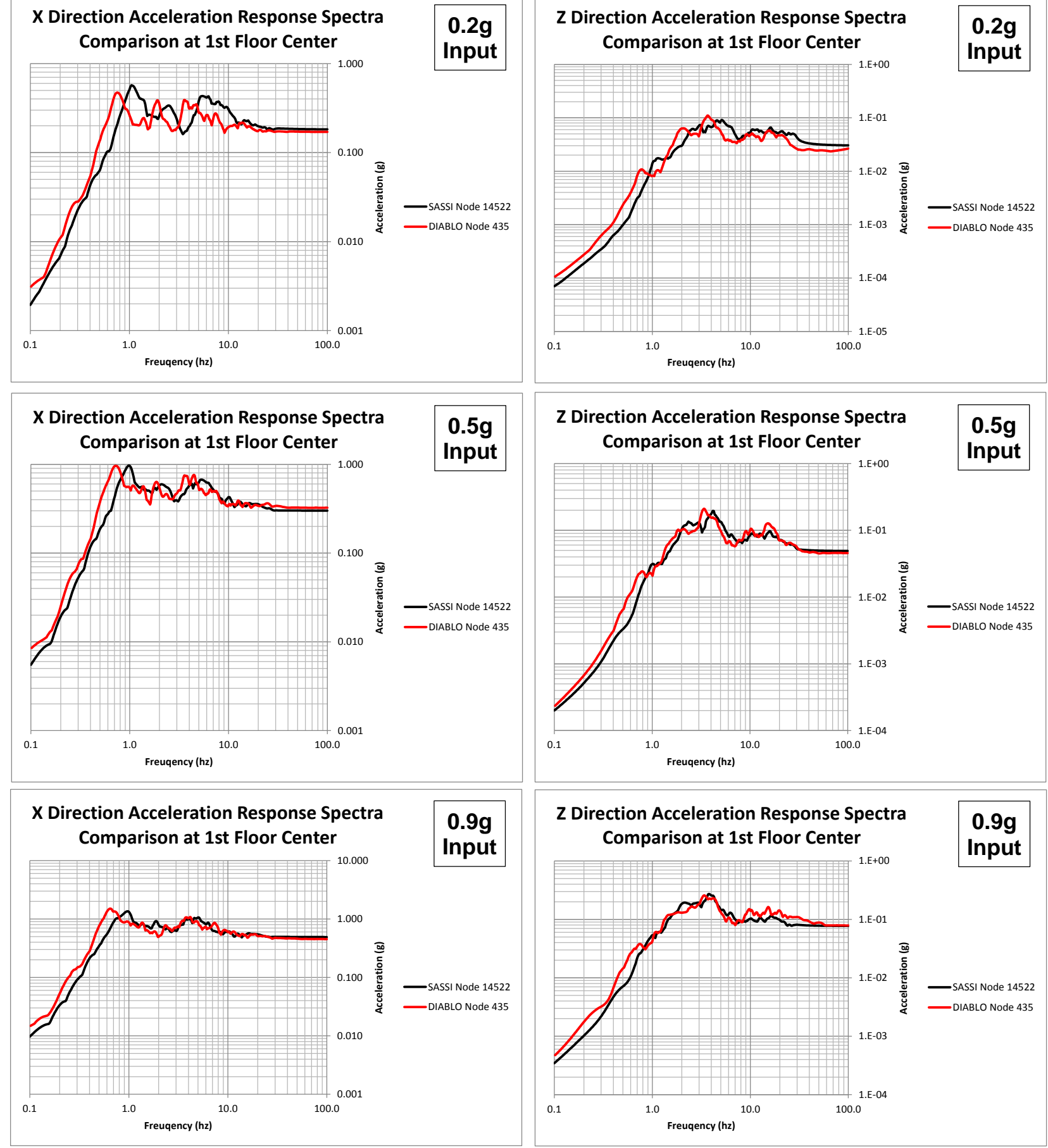

\section{Z Direction Acceleration Response Spectra Comparison at 1st Floor Center}

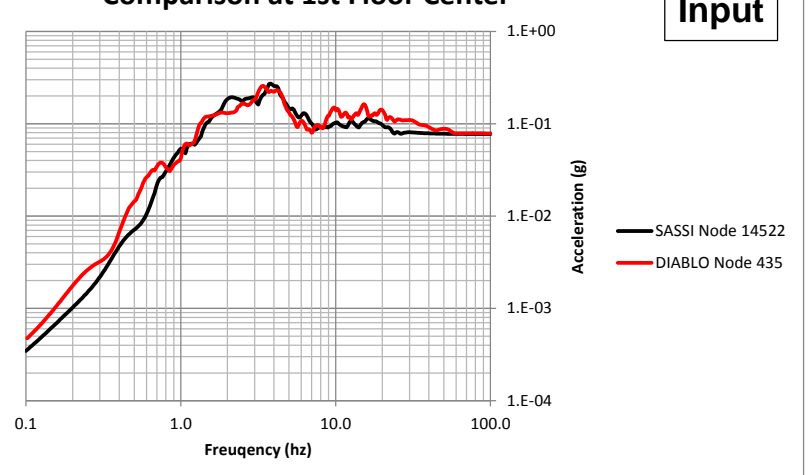

Figure 10 - Comparison of SASSI (black) and DIABLO (red) at the structure edge, on the first floor 
A comparison of the response spectra indicates that the two models produce similar results, except at lower response levels (see Figure 8, Z Direction). The peak frequencies are also sometimes shifted between the two codes. The differences at lower response levels, as well as the peak frequency shifts, can be attributed to the non-linear capabilities of DIABLO in comparison to SASSI. SASSI's equivalent linear approach imposes lower modulus and higher damping everywhere (all frequencies and strains). This leads to under-predicted response in regions of relatively low magnitude, since for this situation DIABLO will be both stiffer and have less damping; hence, soil/structure system resonances will both be shifted in frequency and be less damped.

The modified Bielak method developed by LLNL consists of two modifications (1) a generalization of the Bielak method valid for arbitrarily-thick intermediate regions and nonlinear materials, and (2) a modification to the strain function within the intermediate region that ensures the strain varies smoothly across the intermediate region and across inter-region boundaries. Without this latter modification, the strain within the layer is not a linearly scaled version of the strain within the central region - hence (because the soil is nonlinear) the impedances are mismatched at the layer boundaries. This impedance mismatch causes spurious wave reflection, polluting the solution. To demonstrate the impact of the modified strain, a separate analysis was performed without the modified strain in the intermediate region. In Figure 11, the difference in the spectral response is quite noticeable, especially at the higher frequencies.

Nonlinear soil models naturally form a hierarchy, where models of progressively greater complexity can account for more and more of the response characteristics of actual soils. The Ramberg-Osgood model is a particularly simple form of nonlinear soil model, chosen for the ease by which it is matched to EPRIstyle shear modulus degradation and damping data. The Ramberg-Osgood model was developed primarily to model 1-D response to shear waves and was extended to 3-D response in a very simple manner. As such, it is a member of the family of "tier 0" models that are simple generalizations of 1-D models [23] [24]. It does not incorporate numerous features of advanced soil models, including pressure dependence and cyclic degradation. Thus, it is to be expected that the Ramberg-Osgood results are largely similar to the SASSI results. In fact, this is a significant milestone, as it demonstrates a method whereby, through proper choice of material model and time-domain substructuring approach (the modified Bielak method), benchmark results comparable to SASSI can be generated in the timedomain using modern computational tools. This means that a path forward has been generated whereby the impact of progressively more complex (and physically realistic) models can be evaluated, with traceability to SASSI analysis.

The work also included development of more sophisticated material models that can also be traced to typical EPRI-style test data [25]. In order for the model response to match the data at low strain levels, the "elastic region" of the elastic-plastic response had to be shrunk down to zero size. That is, the model must act plastically even at zero strain. With these modifications, the "Pisano-Jeremic" model so developed was able to reproduce typical experimental results. Additional tuning, which involves adding some small amount of viscous dissipation ${ }^{4}$, further improves the match.

Finally, the work included the development of notional 3D input motion fields compatible with the modified Bielak method [26]. Future work will involve comparing the results of 3D motion input to the equivalent one-dimensional input, as currently used by industry codes such as SASSI.

4 Physically justifiable as the result of pore fluid motion 

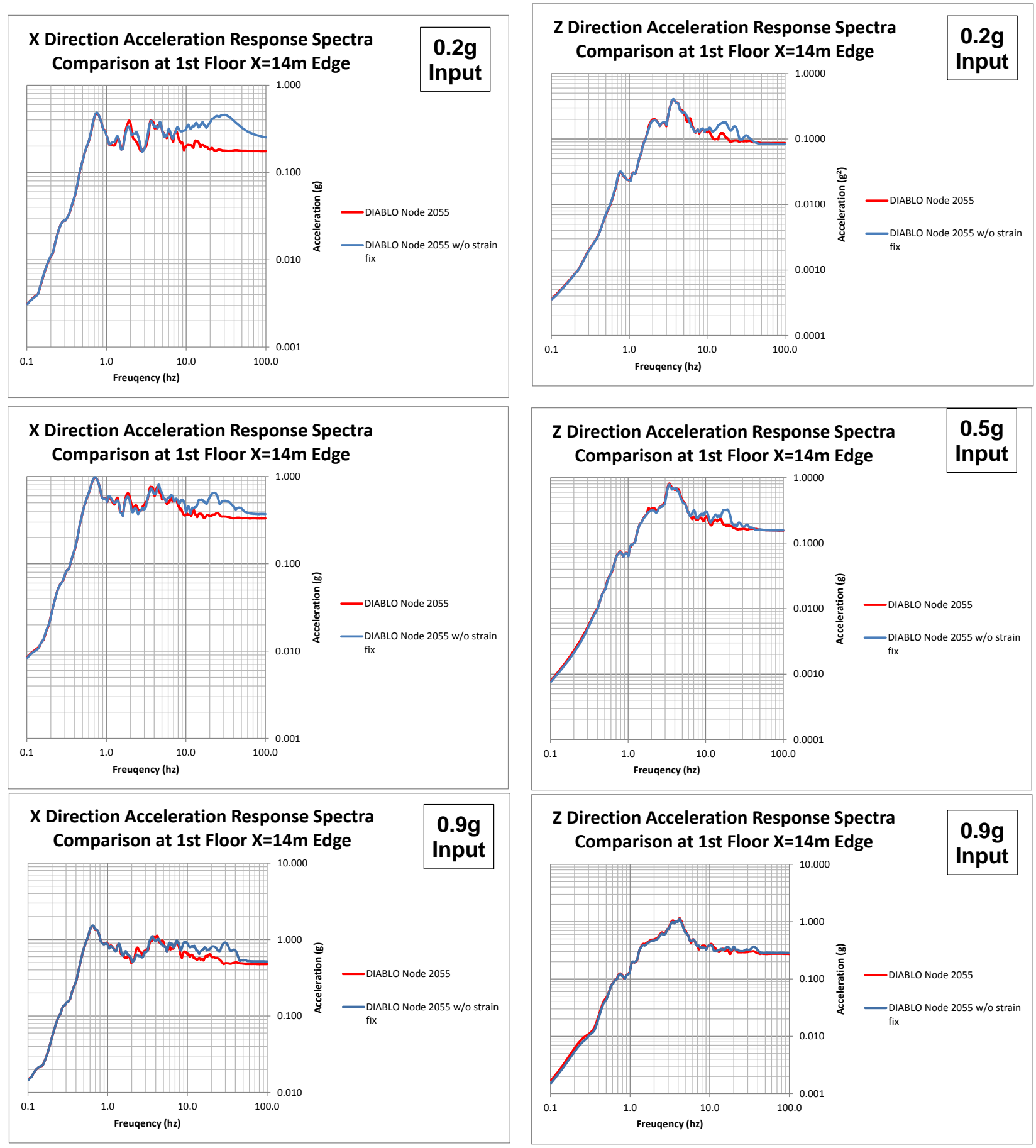

Figure 11 - Comparison of DIABLO with impedance matching (red) and without (blue) for the structure first floor edge

In summary, a generalized time-domain method has been developed which is compatible with material models nonlinear down to zero strain. The utility of this method has been demonstrated by favorable 
comparison to SASSI results for earthquakes with nominal levels of $0.2 \mathrm{~g}, 0.5 \mathrm{~g}$, and $0.9 \mathrm{~g}$. Future work involves incorporation of more physically realistic soil and structural models, and 3D input motions.

\subsection{Development of Advanced Soil Models for SSI Analyses}

The previous section noted the existence of a hierarchy of soil models of progressive complexity. As part of the current study, several advanced soil models have been developed or documented by UC Davis (part of the LBNL team on this project). These models can be incorporated into DIABLO to perform SSI analyses for reactor sites with more complex soil conditions (e.g. incorporating pore water pressure for studying potential liquefaction and pressure dependence). UC Davis and LBNL [25] have developed the traceability of this hierarchy of modern plasticity-based models to the EPRI soil data [4].

\subsection{Development of Three-Dimensional Seismic Input Motions}

Currently, typical SSI analyses are performed using idealized seismic input motions (i.e., by assuming input motions as vertically propagating horizontal shear waves). This simplification can introduce significant inaccuracy in predicting seismic responses - in fact, 3-D seismic wave fields have been shown to increase the nonlinearity of the soil response [27]. As part of this project, a method of developing a three-dimensional input motion field has been developed by LBNL [26]. This methodology is designed to be fully compatible with the non-linear Bielak Method developed at LLNL.

\subsection{Major Observations, Accomplishments, and Conclusions}

- Proper modeling of soils requires accounting for nonlinear behavior even down to very small strains.

- Frequency-domain analysis, as represented by SASSI, cannot properly account for the numerous sources of non-linearity present in soils. The "equivalent linear method", often used in concert with SASSI, can only approximately account for some non-linearities, and can underpredict the structural response if not used very carefully by experts.

- Time-domain analysis, capable of fully accounting for nonlinear response, is the appropriate method to adequately account for the full range of soil-structure interaction.

- LLNL developed a modified Bielak method which facilitates efficient modeling of soil-structure interaction in the time domain, utilizing soil models which are nonlinear even down to zero strain.

- To demonstrate the utility of the time-domain approach, the Ramberg-Osgood soil model was utilized in DIABLO to model nominal earthquakes with intensities of $0.2 g, 0.5 g$, and $0.9 g$. The results were similar to results obtained via SASSI.

- The results of the benchmarking calculations in this study verify the adequacy, in the context of simple soil models, of SASSI-calculated seismic response at large amplitudes, but at the same time clearly demonstrate the limitations of the SASSI methodology.

- A hierarchy of more advanced models has been developed, with traceability to EPRI soil test data. In addition, a means to use fully 3-D seismic inputs derived from geologic-scale wave propagation codes has been developed. 


\subsection{Recommendations}

The current pilot study demonstrated that nonlinear hysteretic soil models are capable of performing SSI analyses that are consistent with SASSI results. The method should be further improved and applied by undertaking the following tasks/projects:

- Incorporate representative, modern 3-D plasticity-based soil models in the fully nonlinear, 3-D SSI framework (DIABLO) and assess the fidelity and computational performance compared to the results in this study for a simple soil model.

- Investigate the effect of full 3-D seismic wave inputs in the fully nonlinear SMR simulation framework, and compare to the results in this study for a vertically propagating horizontal shear wave.

- Investigate the effects of other notable sources of nonlinearity, including pore pressure changes, cyclic degradation, and contact/release (geometric nonlinearity).

- Apply the results of these improvements to SMR demonstration plant designs and to existing power plants whose seismic risk level has increased due to the recent NRC/EPRI Study [28]. 


\subsection{Introduction and Objective}

In the design of nuclear facilities, earthquake-related failures of structures, systems, and components (SSCs) are typically a big contributor to the overall potential risk to members of the public. As such, these facilities are designed to withstand seismic loads that are calculated using an analysis and evaluation methodology that considers, among others, the dynamic effect of the presence of large, heavy nuclear facility structures on the free-field seismic ground motion at the facility site. For designing NRC-licensed commercial nuclear power plants and certain DOE-owned nuclear facilities, this effect, called the soil-structure interaction (SSI) effect, is typically determined with the aid of a frequency-domain seismic response analysis code, SASSI (System for Analysis of Soil-Structure Interaction) [1], originally released in 1981 was subsequently augmented several times and published as SASSI 2000, and is now at version 3 [3]. Although it is extensively used in the nuclear industry to simulate seismic SSI phenomena, this code has limitations, some of which can be potentially severe. Two of the primary limitations are: (i) It is restricted to frequency-domain solutions, representing solutions in the time-domain using superposition and (ii) It represents nonlinear soil properties only indirectly, and cannot account for geometric nonlinearity such as sliding and gap formation at the interface of soil and structural elements. The objective of the study reported here is to develop a state-of-the-art nonlinear time-domain dynamic analysis methodology that would either eliminate or reduce these two limitations of the SASSI-based analysis methodology.

The study is a part of the Nuclear Energy Advanced Modeling and Simulation (NEAMS) Campaign of the US Department of Energy, Office of Nuclear Energy (NE-41). Lawrence Livermore National Laboratory (LLNL) led the multi-lab team that included Argonne National Laboratory (ANL), Brookhaven National Laboratory (BNL), Lawrence Berkeley National Laboratory (LBNL), and Oak Ridge National Laboratory (ORNL).

This report describes in detail the activities performed by LLNL, and summarizes the activities by the other four DOE labs. It also discusses the results, draws conclusions, and provides recommendations for future studies.

\subsection{Evolution and Rationale for the Current Work Scope}

The current effort to develop a state-of-the-art nonlinear time domain SSI analysis methodology is part of the Nuclear Energy Advanced Modeling and Simulation (NEAMS) Campaign of the US Department of Energy, Office of Nuclear Energy (NE-71). The work was conducted within LLNL Work Package MS12LL060312, "SHARP Integrated Performance and Safety Codes - Seismic- LLNL" and the associated work packages for ANL, BNL, LBNL, and ORNL. The overall objective and statement of work (SOW), as described in the DOE-NE electronic project management system, PICS-NE, are paraphrased in Section 2.2 below.

As part of the NEAMS campaign, a workshop was conducted to review the state-of-the-art in simulation of seismic response of nuclear power plants, and to identify the technical areas that should be the focus of the current effort for the development of a state-of-the-art nonlinear time-domain SSI analysis methodology. A summary of the workshop discussions and recommendations are presented in Section 2.3 below. Based on these discussions and recommendations, and considering the availability of funds and resources, LLNL, in consultation with BNL and LBNL, developed the current work scope described in Section 2.4 below. 


\subsection{Objective and SOW in DOE's PICS-NE}

\subsubsection{Objective}

The overall objective of the NEAMS-Seismic work packages are to assess, at a high level, the factors affecting the seismic safety vulnerabilities and design performance characteristics of below-grade advanced nuclear reactors using standard frequency-domain modeling codes and tools, and to compare these with results from newly developing codes that incorporate nonlinear, three-dimensional soil structure interactions in the time domain. The comparison will help identify gaps in the models, codes, assessment tools and methodologies in the modeling of reactor design performance in response to seismic excitation. The knowledge obtained in this project will help guide future development of computational models that can be implemented in a variety of computer codes, both in industry and in the research community. Furthermore the comparison will help identify aspects of advanced reactor designs that are most challenging to ensure performance during and after strong ground motion.

\subsubsection{Statement of Work}

The multi-lab team will conduct a high-level assessment of the factors contributing to the seismic safety risk (SSR) of below-grade advanced nuclear reactor designs. This will be achieved by considering, as a standard test case, a simplified reactor design that encompasses key features important to seismic performance. Seismic safety and design performance characteristics of the test case will be evaluated by modeling reactor behavior during strong seismic excitation using a) traditional tools and codes, b) emerging codes that incorporate nonlinear 3-D time-domain analysis that consider the soil-structure interactions and their evolution as a function of time, as well as local amplification of ground motion by site soil response and seismic phase focusing. These model simulation results will form the basis for a comparison among traditional and emerging modeling codes, assessment tools and methods for the evaluation of the response of advanced nuclear reactor designs to strong ground motion. Model simulation results will also be used to highlight areas of reactor design that deserve more scrutiny or that require more refined models to fully characterize seismic risk.

\subsection{Workshop Organization, Summary and Recommendations}

\subsubsection{Workshop Organization}

The workshop was held at Lawrence Livermore National Laboratory (LLNL) on February 27 and 28, 2012. The purpose of the workshop was to review the state of the art in simulation of seismic response of nuclear power plants, with emphasis on structures partially or fully embedded in the surrounding soil. The workshop reviewed current linear frequency-domain numerical methods (using the SASSI software, for example) and emerging time-domain methods, with the objective of identifying gaps in capability that could be addressed by R\&D within the next 3-5 years.

Robert Budnitz of LBNL, who chaired the Workshop, was tasked with keeping the discussions focused and on-track, and assuring that the discussions provided, in the end, what the Workshop objectives were. James Blink of LLNL assisted Dr. Budnitz, served as recorder, and co-authored the workshop report [2].

The workshop was attended by three groups of people: an eight-member expert panel, the LLNL/LBNL project team, and management representatives from DOE and the NEAMS Campaign. The eight experts 
included very senior current practitioners of frequency-domain methods as well as developers and users of emerging time-domain methods. The following is the list of experts:

- Jacobo Bielak

- Justin Coleman

- Carl Costantino

- Robert Kennedy

- Stephen Mahin

- Farhang Ostadan

- John Stevenson

- Andrew Whittaker

Other participants and observers at the workshop included:

- LLNL: David McCallen, Steven Bohlen, Quazi Hossain, Jerome Solberg, Michael Gerhard, Harris Greenberg, and Randy Settgast

- LBNL: Jens Birkholzer, Lawrence Hutchings and Boris Jeremic (Boris is from UC-Davis with a joint appointment at LBNL)

- DOE-NE: Rob Versluis

- ANL: Keith Bradley and William David Pointer (managers of the DOE NEAMS Program)

The LLNL project team provided information, incrementally, to the experts during the month prior to the workshop. This allowed them to become familiar with the project and to gather information for the discussions at the workshop.

\subsubsection{Workshop Summary}

The workshop began with brief introductory presentations by the project Principal Investigator, Steven Bohlen (LLNL), who laid out the workshop's objectives, and Robert Versluis (DOE-NE-41), who provided DOE's perspectives about the workshop scope and how the results would be used. These brief introductions were followed by three extensive technical presentations by members of the LLNL-LBNL project team (David McCallen, Boris Jeremic, and Lawrence Hutchings). Each of the three technical presentations was accompanied by discussion during the presentation, followed by additional discussion following the presentation. Contributions to these discussions arose not only from the eight invited experts, but also from the other attendees. These presentations and the discussions surrounding them consumed most of the first morning.

After these presentations and discussions, shorter presentations were made by four of the experts (Andrew Whittaker, Justin Coleman, Stephen Mahin, and Jacobo Bielak). In general, these presentations informed the project team about related current and past work at their institutions, including a brief description by Jacobo Bielak of the Domain Reduction Method (DRM), a time-domain methodology for modeling structure-soil-structure interaction in heterogeneous soil-structure systems subjected to a prescribed near-field earthquake ground motion environment.

The workshop then continued with broad group discussion, participated in by both the eight experts and the other attendees, to address the main issue before the group, namely to agree as to the identification of the "gaps" in current capability, and to proposals to address each of those "gaps" through $R \boldsymbol{R} \boldsymbol{Q} \boldsymbol{D}$. While some of the discussion inevitably touched on suggestions as to the relative 
priorities of the proposed R\&D, the focus in this session was more on identifying outstanding issues rather than on assigning priorities. This group discussion consumed most of the first afternoon.

The second day began with an extensive executive session of the eight invited experts. At the experts' request, four LLNL-LBNL team members (James Blink, Steven Bohlen, Robert Budnitz, and David McCallen), attended to document the deliberations and prepare for a report-out session to the full group. This session focused on prioritization, and specifically on developing a prioritized list of "gaps" and proposals for R\&D to address each gap. The full group discussed the prioritized list and R\&D proposals during the "report-out" session that was attended by the experts, the DOE representatives, and the full LLNL-LBNL project team. This report-out session resulted in some modifications to the way the prioritized list of gaps had been explained in the earlier executive session; the modifications were mostly in the nature of more complete explanations of some of the ideas, so as to assure that ambiguities were minimized and that the R\&D path forward was clear to the DOE and project team attendees.

\subsubsection{Workshop Conclusions and Recommendations}

The eight experts developed both their list of gaps and their recommendations for the R\&D necessary to close each of the gaps. The focus was on R\&D that the experts judged to be feasible over a 3-5 year period. The R\&D recommendations attempted to account for work underway in the broad (international) community, rather than restricting it in any geographical or institutional way. Also, the experts did not specifically express where or by whom their recommendations for R\&D could be carried out. It was sufficient, in their minds, that the R\&D be judged to be fully feasible, within the near future, by organizations and individuals with state-of-the-art knowledge relative to linear and non-linear geometries and to material behavior subjected to dynamic cyclic loads applied to a finite element solution in the time domain. It was also noted that any resultant analytical tasks developed should be executable by knowledgeable structural design and analysis organizations or individuals.

There was some limited discussion of the ongoing work now underway as part of this DOE-NEAMS work package. It was recognized that the current work package comprises some of the initial steps in executing the 3-5 year effort; the LLNL-LBNL team in turn noted that the expert recommendations were largely consistent with the planning documented in PICS-NE for this work package, although the planning is clearly neither as extensive nor as detailed, and only includes work in fiscal year 2012.

The experts recommended six areas of R\&D work. The experts determined that two of the areas are of high priority, and that important parts of these two could be executed in parallel.

- Nonlinearities Associated with the Interface of the Structure and Soil

- Arbitrary, Spatially-Distributed, Wave Fields

A logical follow-on to the two top priority tasks is a series of calculations with the time-domain approach, which would include nonlinear soil-structure behavior, local heterogeneities, and spatiallydistributed wave fields. The results could then be compared to the corresponding SASSI results in order to determine the practical significance of these effects, which cannot be captured by SASSI. (The experts noted that the challenge will be to determine the correct answer to compare to the two model results.) 
The other four areas that the experts recommended for R\&D can also be worked on in parallel with each other, but are largely not within the current scope of this work package.

- Nonlinear Hysteretic Models of Structural Elements

- Seismic Isolators

- Nonlinear Soil

- Internal Fluid-Structure Interaction

In addition to these six areas, the experts did not explicitly recommended another area discussed extensively during the Workshop, in part because the topic was somewhat out-of-scope. This topic is:

- Methods for Developing Realistic Three-component, Time-domain "Records" from a Scattered Wave field, as Input to Soil Models.

Although the topic was outside the Workshop's narrow scope, there was a sentiment expressed during the Workshop that ultimately this type of modeling work on the input motions to the soils model will be an important constituent of the long-sought realistic numerical model of the entire "system".

\subsubsection{Nonlinearities Associated with the Interface between the Structure and the Soil}

One of the two recommended high priority tasks was to develop time-domain analysis methods and then software to handle local nonlinearities associated with the interface of the soil and the structure (geometric non-linearity). These nonlinearities include the following:

- Local high compression stresses in the area of interface between the soil and structure (localized soil nonlinearities are the results of these high stresses)

- Slip due to shear forces

- Gaps due to inability to transfer tension

- Buoyancy effects for embedded structures.

This high-priority task should begin by comparing a linear finite element algorithm in the time domain to SASSI (frequency domain) results. This comparison analysis should assume linear and elastic geometric and material soil and building properties. It should also strive to develop an explanation of any differences in results between the time-domain results and the SASSI results. This comparison should be performed for three levels of excitation; $0.2 \mathrm{~g}, 0.5 \mathrm{~g}$ and $1.0 \mathrm{~g}$ peak ground accelerations.

The experts emphasized that the software resulting from this task should not omit any existing SASSI capability. For example, when seismic waves reflecting from the structure encounter the boundaries of the SASSI calculation, these waves do not reflect back toward the structure; the time-domain software should have similar characteristics for its domain boundaries at the sides and the bottom.

The experts recommended that the software be developed in an incremental fashion. The first calculation is intended to be a verification of the time-domain method. It would be comparable to a SASSI calculation if it uses a structure that is "glued" to an equivalent-linear soil model, with the seismic wave being a vertically propagating, horizontally polarized, shear wave. The "glued" interface will prevent sliding and gaps that cannot be calculated by SASSI, and hence are undesirable in a verification calculation against an alternative code. The model should calculate in-structure response spectra at 
several locations, and should be compared with SASSI results at several ground motion amplitudes. The experts advised doing the initial calculation with a half-space (homogeneous) soil, and then proceeding to a layered soil structure. It was noted that previous efforts in this area have taught the community that care must be taken in areas such as damping in order that a time-domain model can successfully reproduce SASSI results.

It is important that the coupling between the two horizontal directions and the vertical direction during the sliding and gapping is maintained. Some programs can handle the sliding and gapping, but with no consideration for coupling, making the results less decisive.

Once the time-domain model is verified to reproduce SASSI results, the suite of calculations (at several ground motion amplitudes and for single and multiple soil layers) should be repeated including, incrementally, the above nonlinearities.

The experts discussed the buoyancy nonlinearity that arises when a structure is embedded, even partially, below the water table. The frictional force preventing slipping of the structure along the soil interface (for a shallowly-embedded structure) is the coefficient of friction times the normal force, and the normal force is the downward weight of the structure less the upward buoyancy of the wet soil material displaced by the structure. For a structure with a considerable "empty" volume, the buoyancy can be a significant fraction of the weight, and thus can lead to possible slipping in a simple calculation. However, it needs to be realized that the fluid in the soil cannot support shear stress, and hence a realistic model that accounts for potential slipping must account for the soil deformation rather than treating the structure and soil as two rigid bodies that slip against each other.

For buoyancy effects, it is important to separate the issue of pore pressure and liquefaction from buoyancy and vertical inertia forces. The latter is more relevant to nuclear structures, and for some designs this has become a barrier. The current design approach is a simple hand calculation of static buoyancy force less vertical inertia. This is overly conservative given the short duration of peak accelerations. A rigorous 3D time-domain analysis will permit uplift and should provide a more realistic estimate of uplift even if the current hand calculations show a factor of safety less than unity.

\subsubsection{The Use of Arbitrary, Spatially-Distributed Wave Fields as Seismic Input}

The second of the two recommended high priority tasks was to develop time-domain analysis methods and then software to handle fully three-dimensional incident wave fields that are produced from measurements or from models of propagation of seismic waves from a seismic source such as a fault rupture. These wave fields can be spatially distributed at the model boundaries, with varying phase and amplitudes from point to point. As in the other top priority task, the work should be incremental. SASSI can calculate response to planar waves at various inclination angles to the vertical as well as response to surface waves, and the time-domain model should be run to compare with SASSI results. The experts, however, noted that although inclined plane wave calculations will be useful, the users of the models should be aware that actual seismic wave fields are very complex.

The effort should start with free-field motion without any structure present. The free-field motion must be carefully examined to evaluate the contents of the wave field frequency-by-frequency, and the coherency of the motion point-by-point. Both uniform and layered sites must be considered. Without a 
rigorous assessment of the wave field in the free-field, any study of the effect on the structure may become too complex to understand.

As for the other high priority task, the model should calculate in-structure response spectra at several locations, and should be compared with SASSI results at several ground motion amplitudes. The experts advised doing the initial calculations with a half-space (homogeneous) soil, and then proceeding to a layered soil structure.

\subsubsection{Nonlinear Hysteretic Models of Structural Elements}

Advanced design features associated with advanced light water reactors (LWRs) and small modular reactors (SMRs) can improve design margin and result in lower cost systems. An important recent example is a steel-concrete (SC) wall in which concrete is placed between two steel plates with multiple tie bars. Other features worthy of additional study include low-rise shear walls and braced frames used in traditional construction. The performance of these features can sometimes be non-linear, and the time-domain approach is thus well suited to analyze their performance. Current SASSI capabilities allow only for linear elastic analyses to be performed.

In discussing these advanced design features, the experts noted that a) recent experience with SC walls reveals that the confinement of the concrete during the elevated temperature curing time, followed by shrinkage as the concrete cools, results in cracked concrete that has less of a stiffness change during the cyclic loading of a seismic event, and b) recent tests of low-rise reinforced concrete (RC) walls have generated data different than that predicted with advanced models and computer codes.

The work in this task involves developing realistic constitutive models, benchmarked with appropriate data, which can capture the behavior of these structural elements during earthquakes. There is a need for understanding the behavior for both design-basis earthquakes and earthquakes whose size is substantially beyond the design basis. Formerly, this was a formidable modeling challenge because of limitations in computer size and power, but that limitation has been swept away, and modern algorithm development has also provided advanced, but non-validated, tools for the modeler. The experts judged that this area is ripe for a major development. The development will, in turn, enable the use of these advanced elements in reactor design in situations now precluded because they cannot be analyzed with sufficient accuracy, and hence a regulatory safety case cannot be supported at this time. It will also enable changes in industry consensus codes and later in the safety regulations themselves. These changes are not presently allowed, partly due to the inability of current methods to perform sufficiently realistic analyses.

\subsubsection{Modeling Seismic Isolators}

The experts concluded that the time-domain SSI analysis capability should include the capability to calculate the performance of seismic isolators. Both elastomeric and sliding isolators should be considered. The models should address the effects of energy dissipation that lead to changes in the hysteretic behavior of the components.

A good deal of research work is already underway to understand isolator behavior, including both experimental studies and the development of advanced analytical methods. The experts emphasized that continuing support for this work is important. One major theme of the ongoing work, which requires a time-domain model, is on understanding the behavior of the isolators when subjected to 
extreme earthquake motions well-beyond the design basis, with an emphasis on understanding what happens if an isolator system were to reach its boundary (or "moat"). This can lead to large impact forces, and in turn, transmit large motions up into the structure above the isolators. Work on this important issue, both experimental and analytical, is already underway but needs additional support. A second area is consideration of how horizontal seismic motions coming into the facility can produce vertical motions due to the rocking flexibility of the isolation system. A third area is working on how isolator performance in nuclear reactor systems can be understood well enough to allow its inclusion in the consensus design codes and later in the nuclear-safety regulatory process [29]. This body of recommended isolator R\&D work, taken as a whole, is intended to expedite the acceptance of base isolation of nuclear reactor facilities as a viable and useful option for the designer.

\subsubsection{Modeling Nonlinear Soil}

The experts emphasized that the inability to model nonlinear soil behavior is a significant limitation of the elastic SASSI model, and that care must be taken in interpreting SASSI results because, at high shear strain levels, the equivalent linear soil model used in SASSI overstates resonances in response spectra and understates the ability of the soil to pass high frequencies to the structure. A time-domain model can directly calculate nonlinear response of soil to seismic waves; however, the experts strongly cautioned that care must be taken to use nonlinear constitutive models that capture the behavior of real soils. They further noted that the simplest useful 3D constitutive model, an elastic Coulomb-Mohr model, does not correctly capture nonlinear behavior. It is very important that down-hole array data be used and that the model is validated based on free-field motions, before the model is applied to SSI calculations used for design or regulation.

The experts concluded their discussion of this task with a strong call for verification of soil models, and where possible, validation against data, with associated management of uncertainties. Also, a few experts noted that since equivalent linear properties in SASSI are established from nonlinear mechanical properties, care must be taken with nonlinear constitutive models, but arguably the equivalent linear properties are even more uncertain.

\subsubsection{Internal Fluid-Structure Interaction}

The experts noted that some SMR design concepts include pools of water. They emphasized the need for a time-domain approach to enable simulation of the sequential interaction of the structure with the fluid (causing both pressure waves and fluid movement), and then the interaction of the fluid back with the structure. They noted that in off-normal scenarios, the model should be able to calculate the behavior of the system under seismic loading after some of the water has been vaporized. Finally, they noted that the model must properly include the nature of the physical contact of the fluid and the structure.

\subsection{Current Work Scope}

The LLNL Team, in consultation with the BNL and LBNL Teams, considered the deliberations of the Workshop participants and the Workshop recommendations, along with the availability of project funds and resources, and decided to undertake the following major task items for the current phase of the project:

- Development of a Finite Element Model for a Typical Modular Reactor Building 
- Development of Time Domain Finite Element Technology for SSI analysis

- Development of Input motions

- Development of Material Models

- Time-domain SSI analysis and comparison with SASSI results

- Identification of technical issues and recommendations for future work

As shown in Section 3.0, some of these tasks were divided into more than one sub-task. 


\subsection{Results of the Study}

\subsection{Development of a Finite Element Model for a Typical Modular Reactor Building}

\subsubsection{Reactor Building Configuration and Development of the Finite Element Model}

It was desired to develop a structural model including the soil and related features that was simple, but at the same time revealed typical features of Small Modular Reactor (SMR) designs, especially those embedded in the soil. The features that were considered important were:

- A reactor building structure more than one-half embedded in the soil

- Heavy base floor (i.e., the 'basemat')

- A number of floors (at least 3)

- A cylindrical reactor vessel supported on a pier.

- A feature simulating the mass of an internal water tank (not to include the effect of sloshing).

- A number of "stick models" representing installed equipment

- A surrounding soil region consisting of a number of layers preferably derived from an actual site analysis.

Following a discussion of LLNL and ORNL staff on whether the basic Reactor Building structure should be rectangular or circular in shape, a rectangular Reactor Building structure surrounding an inner cylindrical pier supporting the reactor vessel was selected. The chosen configuration, because it includes a mixture of cylindrical and rectangular features, provides a good test of the computational framework. 
The Reactor Building structure is illustrated in Figure 12. Note the position of the ground level between floors 2 and 3. Except where noted, the structure is assumed to be composed of reinforced concrete.

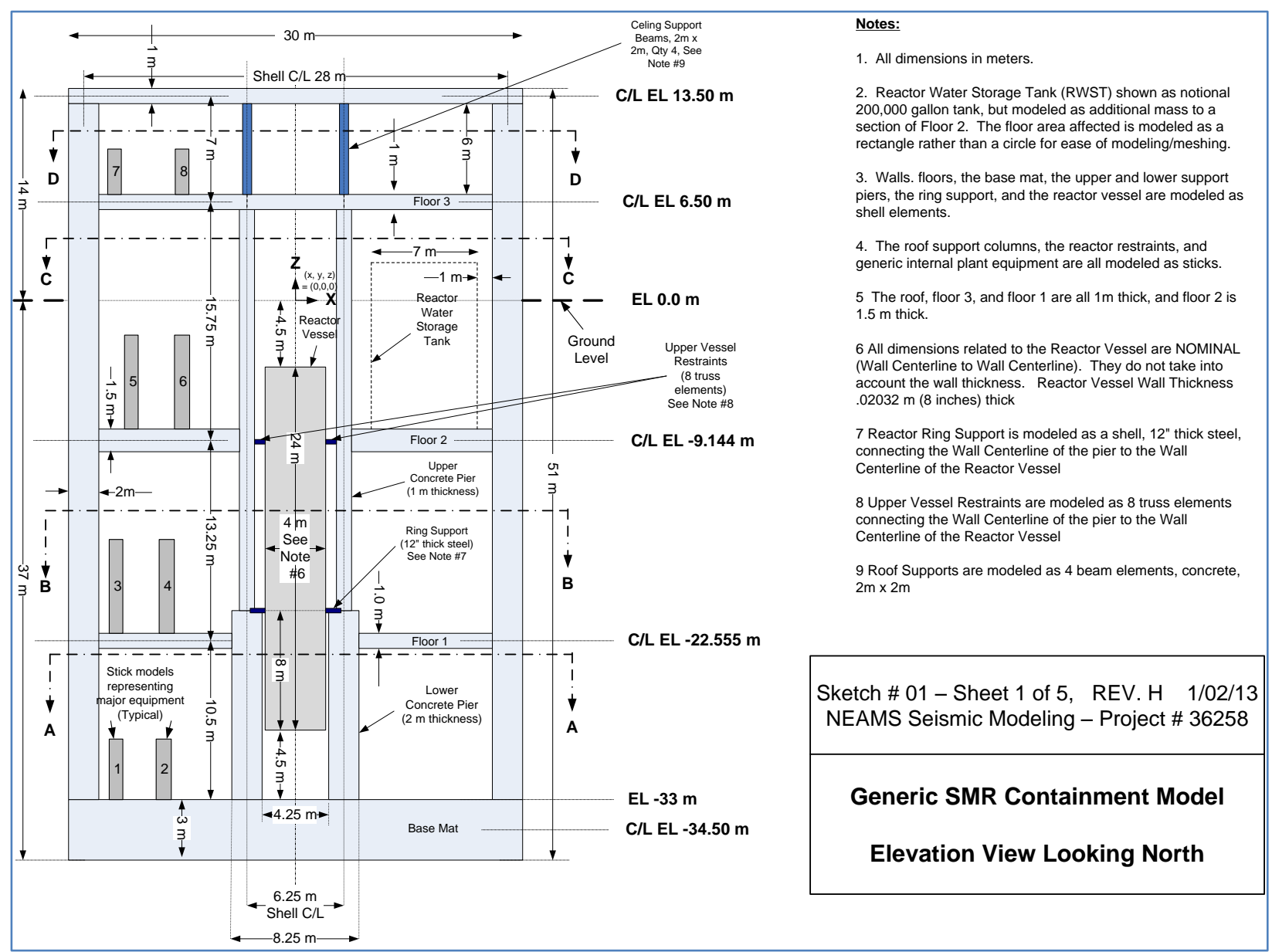

Figure 12 - Reactor and Containment Structure, Sheet 1, Elevation View 
Sectional views of the basemat, and Floors 1 through 3 are shown in Figure 13, Figure 14, Figure 15, and Figure 16, respectively. The reactor is attached to the pier structure via a support ring modeled by shell elements, and restrained from rotation about the $X$ or $Z$ axes by truss elements aligned with floor 2 . The pier extends all the way to floor 3 , with the roof supported by column elements between floor 3 and the ceiling.

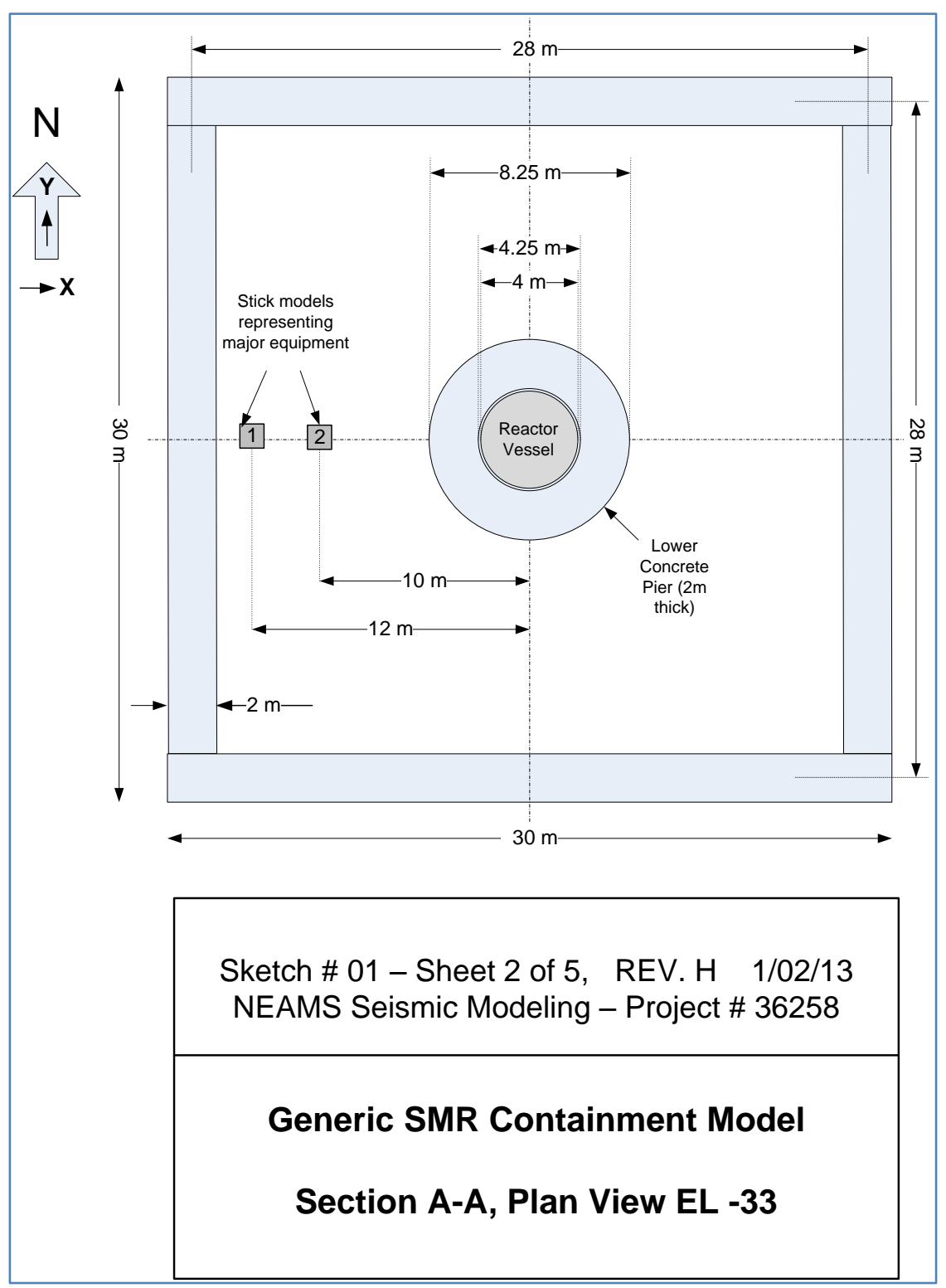

Figure 13 - Notional Reactor Model, Sheet 2, Section View Downward towards Base Mat 


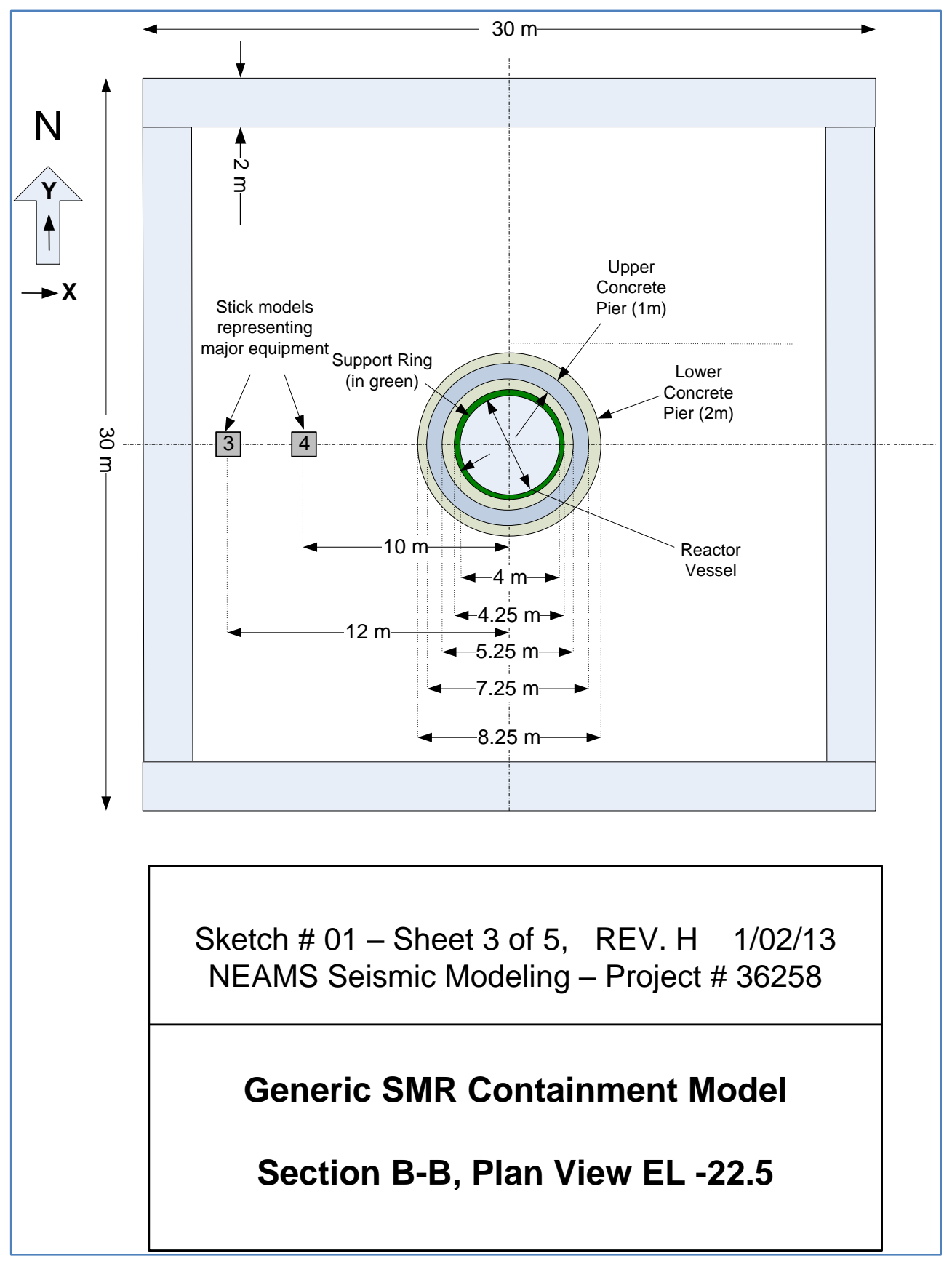

Figure 14 - Reactor and Containment Structure, Sheet 3, Section View Downward towards Floor 1 


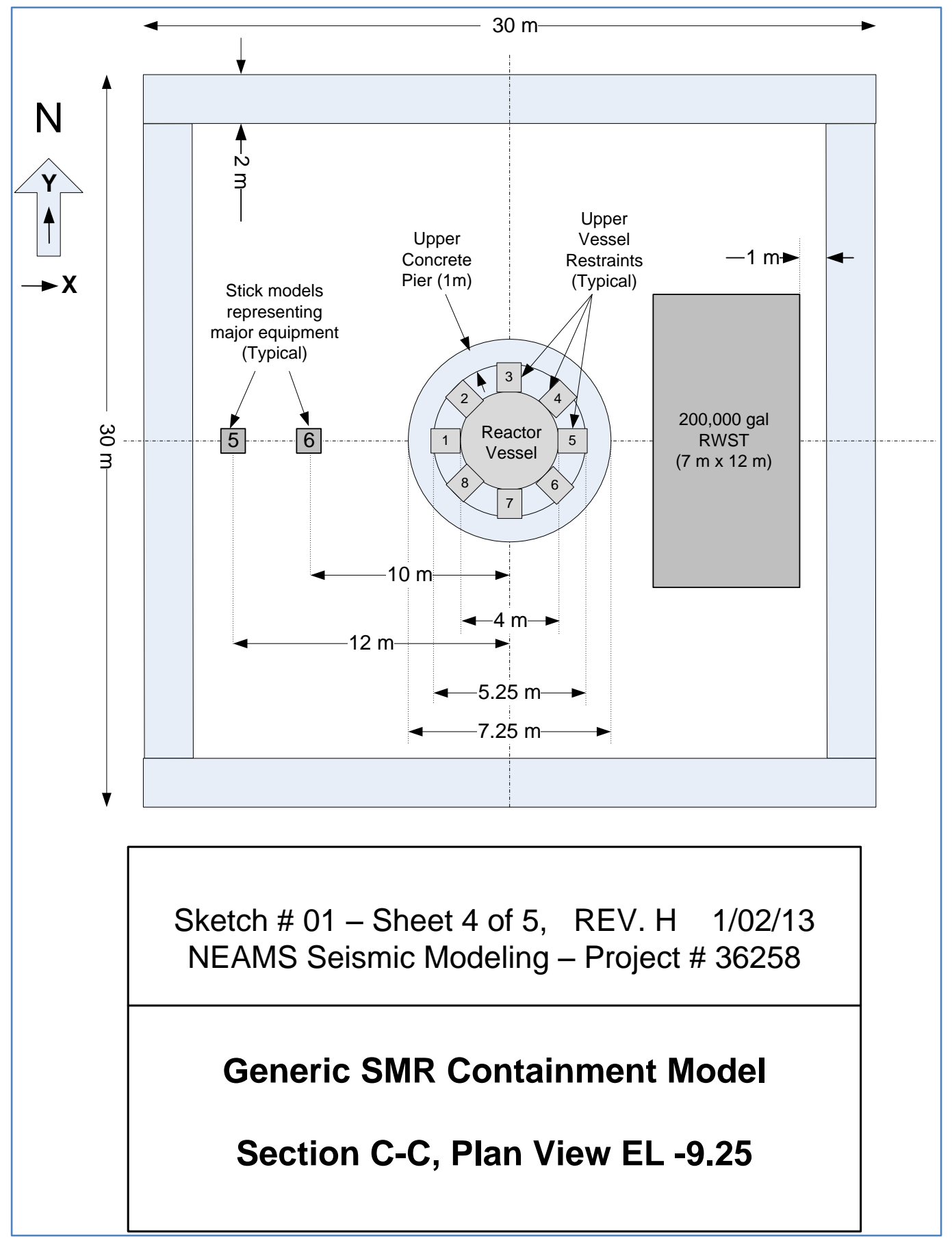

Figure 15 - Reactor and Containment Structure, Sheet 4, Section View Downward towards Floor 2 


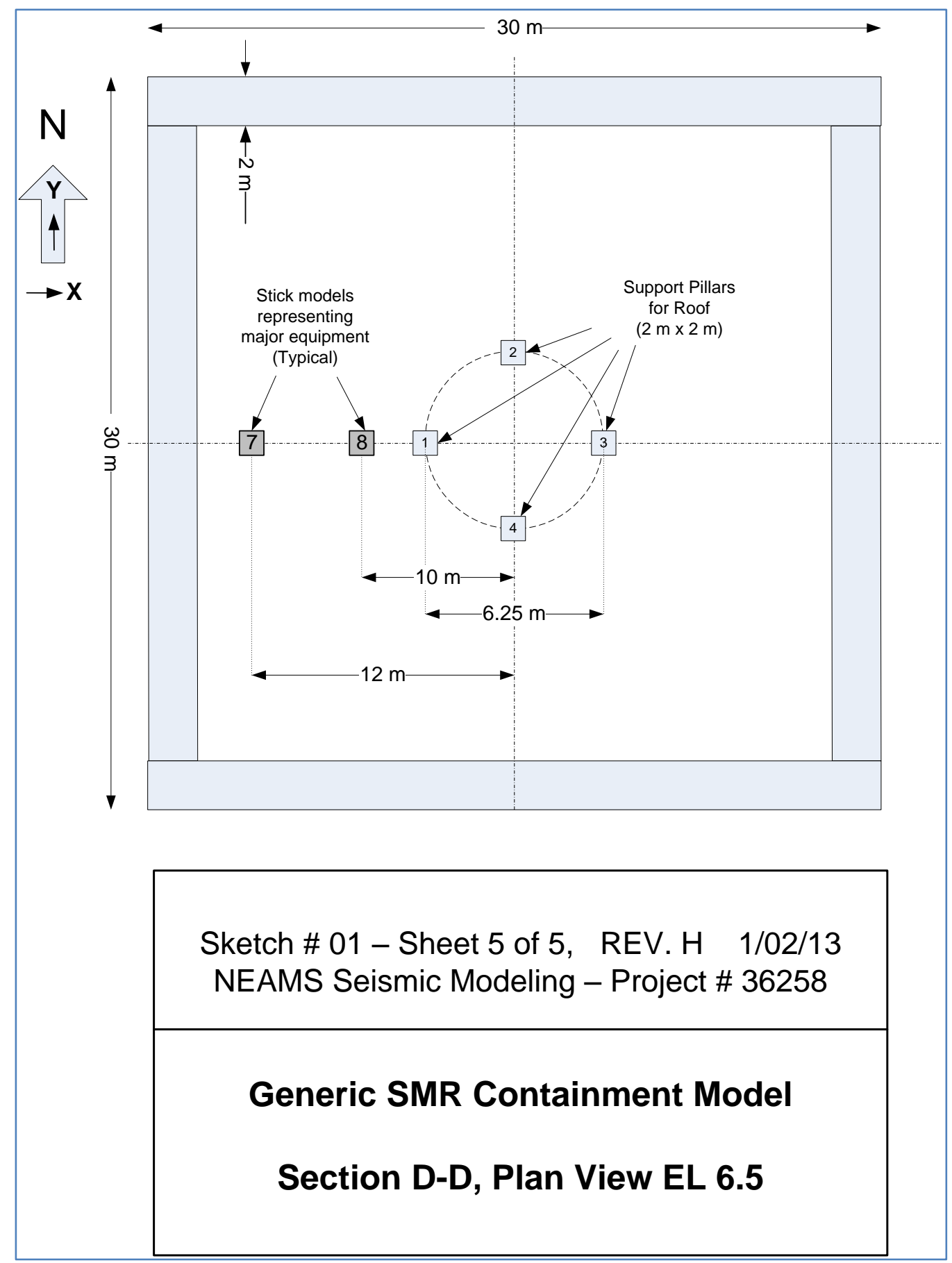

Figure 16 - Reactor and Containment Structure, Sheet 5, Section View Downward toward Floor 3

Eight (8) "lumped mass sticks" were included in the Reactor Building model to represent typical major pieces of equipment. Mass is added to all of the major structural elements in order to account for distributed loads (minor equipment). The properties of the structural elements are summarized in Figure 17. The properties of the beam and stick models are summarized in Figure 18. The stick model properties were calculated based upon the table included in Figure 19. Notes related to the materials are included in Figure 20. 


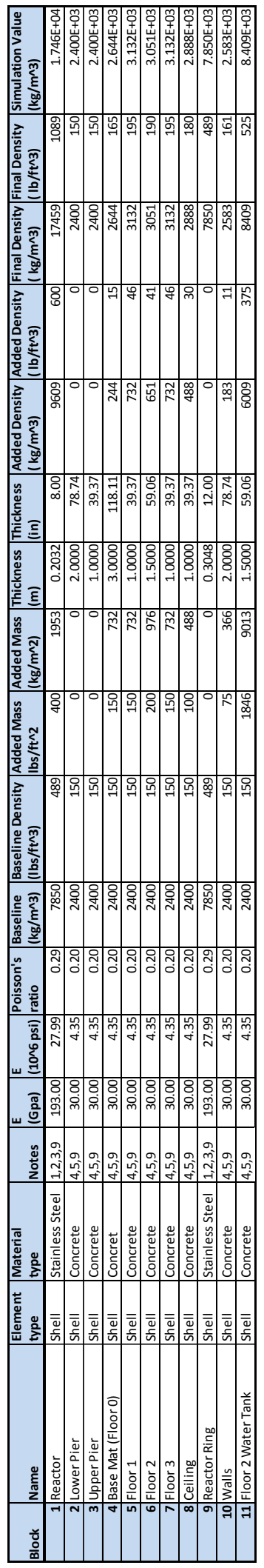


Figure 17 - Structural Elements - Material and Cross-Sectional Properties

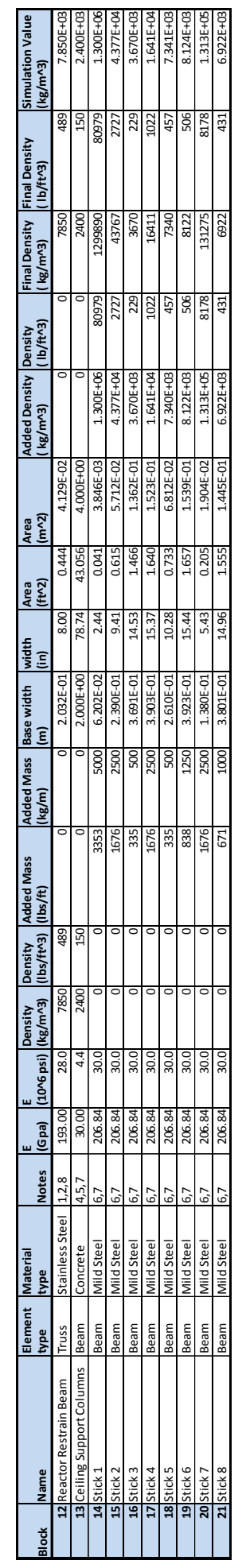

Figure 18 - Beam and Stick Elements Material and Cross-Sectional Properties 


\begin{tabular}{|c|c|c|c|c|c|c|c|c|}
\hline Stick Number & $\begin{array}{c}\text { Target } \\
\text { Frequency, } f\end{array}$ & Length (m) & $\begin{array}{l}\text { Distributed Weight } \\
\text { (kg/meter) }\end{array}$ & $\begin{array}{l}\text { Moment of Inertia } \\
\left(\text { meter }^{4}\right) \text {, assuming } \\
\mathrm{E}=30 \times 10^{6} \mathrm{psi}\end{array}$ & $\begin{array}{l}\text { No. of Nodes including } \\
\text { the top and the Bottom }\end{array}$ & \begin{tabular}{|c|} 
Equivalent base \\
width ( $\mathrm{m}$ ) assuming \\
square
\end{tabular} & $\begin{array}{c}\text { Equivalent Area ( } \mathrm{m}^{\wedge} \mathbf{2} \text { ) assuming } \\
\text { square }\end{array}$ & $\begin{array}{c}\text { Equivalent Mass } \\
\text { Density } \\
\left(\mathrm{kg} / \mathrm{m}^{\wedge} \mathbf{3}\right)\end{array}$ \\
\hline 1 & 1 & 2 & 5000 & $1.23 \mathrm{E}-06$ & 3 & $6.202 \mathrm{E}-02$ & 3.85E-03 & $1.300 \mathrm{E}+06$ \\
\hline 2 & 21 & 2 & 2500 & $2.72 \mathrm{E}-04$ & 3 & $2.390 \mathrm{E}-01$ & $5.71 \mathrm{E}-02$ & $4.377 \mathrm{E}+04$ \\
\hline 3 & 7 & 8 & 500 & $1.55 \mathrm{E}-03$ & 5 & $3.691 \mathrm{E}-01$ & $1.36 \mathrm{E}-01$ & $3.670 \mathrm{E}+03$ \\
\hline 4 & 14 & 4 & 2500 & $1.93 \mathrm{E}-03$ & 3 & 3.903E-01 & $1.52 \mathrm{E}-01$ & $1.641 \mathrm{E}+04$ \\
\hline 5 & 14 & 4 & 500 & $3.87 E-04$ & 3 & $2.610 \mathrm{E}-01$ & $6.81 \mathrm{E}-02$ & $7.341 \mathrm{E}+03$ \\
\hline 6 & 5 & 8 & 1250 & $1.97 \mathrm{E}-03$ & 5 & $3.923 \mathrm{E}-01$ & $1.54 \mathrm{E}-01$ & $8.124 \mathrm{E}+03$ \\
\hline 7 & 7 & 2 & 2500 & $3.02 \mathrm{E}-05$ & 3 & $1.380 \mathrm{E}-01$ & $1.90 \mathrm{E}-02$ & $1.313 \mathrm{E}+05$ \\
\hline 8 & 21 & 4 & 1000 & $1.74 \mathrm{E}-03$ & 3 & $3.801 \mathrm{E}-01$ & $1.44 \mathrm{E}-01$ & $6.922 \mathrm{E}+03$ \\
\hline
\end{tabular}

Figure 19 - Stick Element Worksheet

\begin{tabular}{|c|c|}
\hline Note & Description \\
\hline 1 & Stainless Steel 304 - Young's Modulus 193 Gpa, Poisson's Ratio 0.29 - http://www.azom.com/article.aspx?ArticleID=965 \\
\hline 2 & Using the same density for steel througout - $7.85 \mathrm{~g} / \mathrm{cc}$ (Stainless Steel tends to be a little bit heavier - $8.0 \mathrm{~g} / \mathrm{cc}$, but it does vary) \\
\hline 3 & Poisson's ratio for Stainless Steel - $\mathbf{0 . 2 9}$ - http://asm.matweb.com/search/SpecificMaterial.asp?bassnum=MQ304A \\
\hline 4 & Density for Reinforced Concrete - used $\mathbf{2 4 0 0 ~ k g / m ³ , ~ f r o m ~ R a n d y ~ B e l l e ' s ~ t a b l e , ~ r o u n d ~ n u m b e r ~ i n ~ S I ~ u n i t s ~}$ \\
\hline 5 & Young's Modulus for Concrete - used round number in SI units $30 \mathrm{Gpa}$ \\
\hline 6 & Mild Steel used standard 30e6 psi for Young's Modulus \\
\hline 7 & All beams are calculated using $\mathbf{4 X 4}$ Gauss Quadrature in the cross-section using a Hughes-Liu beam formulation with linear kinematics \\
\hline 8 & All Trusses are calculated using $\mathbf{1 x 1}$ Gauss Quadrature in the cross-section with linear kinematics \\
\hline 9 & All Shells are calculated using $\mathbf{3}$ Gauss Points through the thickness using a Hughes-Liu shell formulation with linear kinematics \\
\hline
\end{tabular}

Figure 20 - Notes related to Material and Sectional Properties

A finite element mesh/model was developed in CUBIT [30] to represent the structure. The resulting finite element model was shared between SASSI and Diablo analyses. In order to align the building finite element grids with soil layers, the vertical dimension of the mesh was chosen as shown in Figure 21. The finite element model of the reactor structure and equipment without the surrounding soil, the outer walls, or the internal piers is illustrated in Figure 22. In this figure, the reactor vessel itself, the reactor restraint beams, and the reactor support ring can clearly be seen. In Figure 23, the upper and lower piers, the stick models, the section of floor 2 where additional mass is added to simulate the effect of the reactor pool, and the upper and lower piers can be seen. The resulting finite element mesh consists of 9463 nodes and 9674 elements, of which 9542 are shell elements and 132 are beam/truss elements. 
Vertical mesh size in Reactor

each section

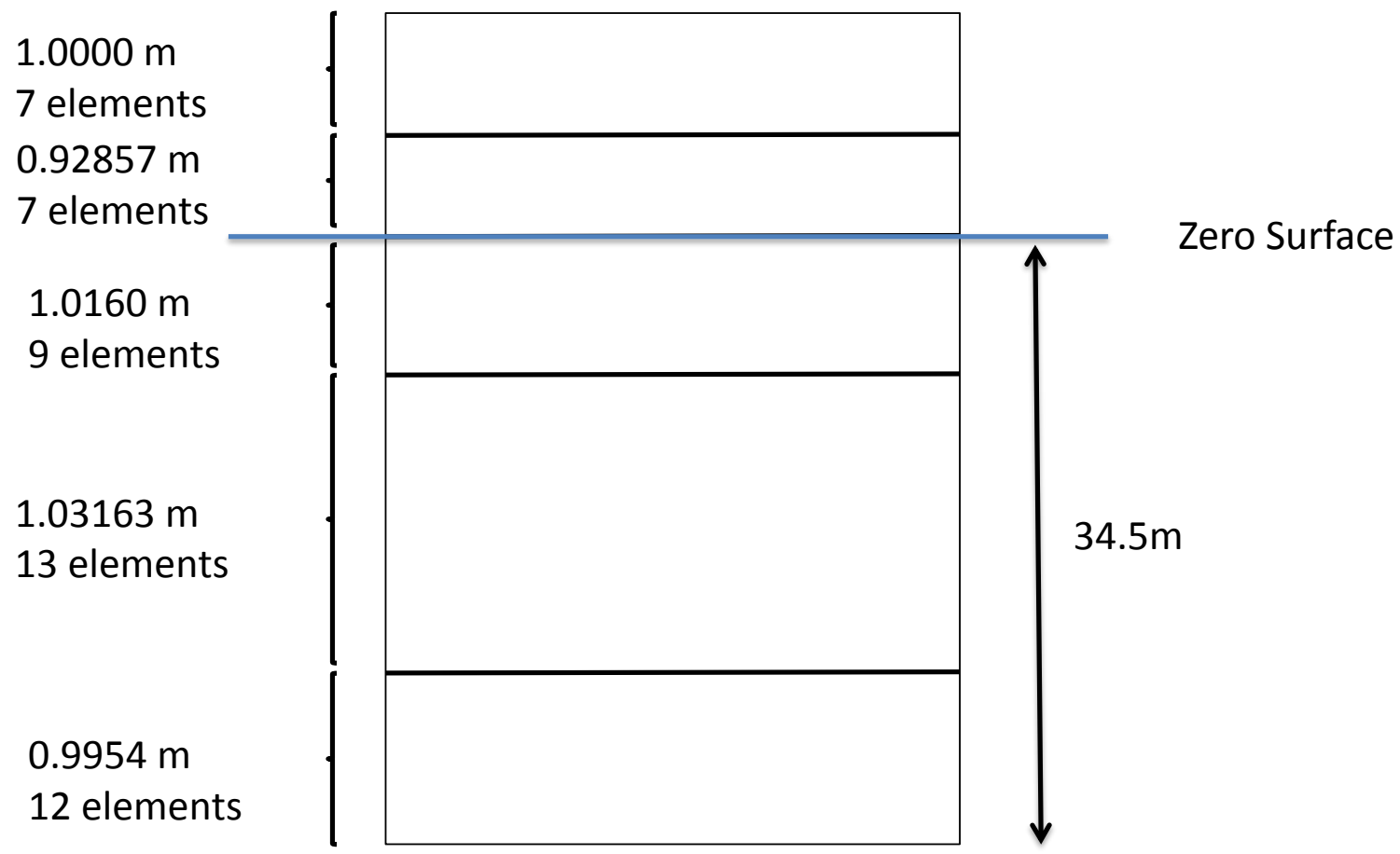

Figure 21 - Vertical Mesh Density. The dimensions on the left are the size of each element. The top of the graphic is the centerline of the roof, the bottom of the graphic is the centerline of the basemat, and the zero surface is shown as "ground level" on Figure 12. 

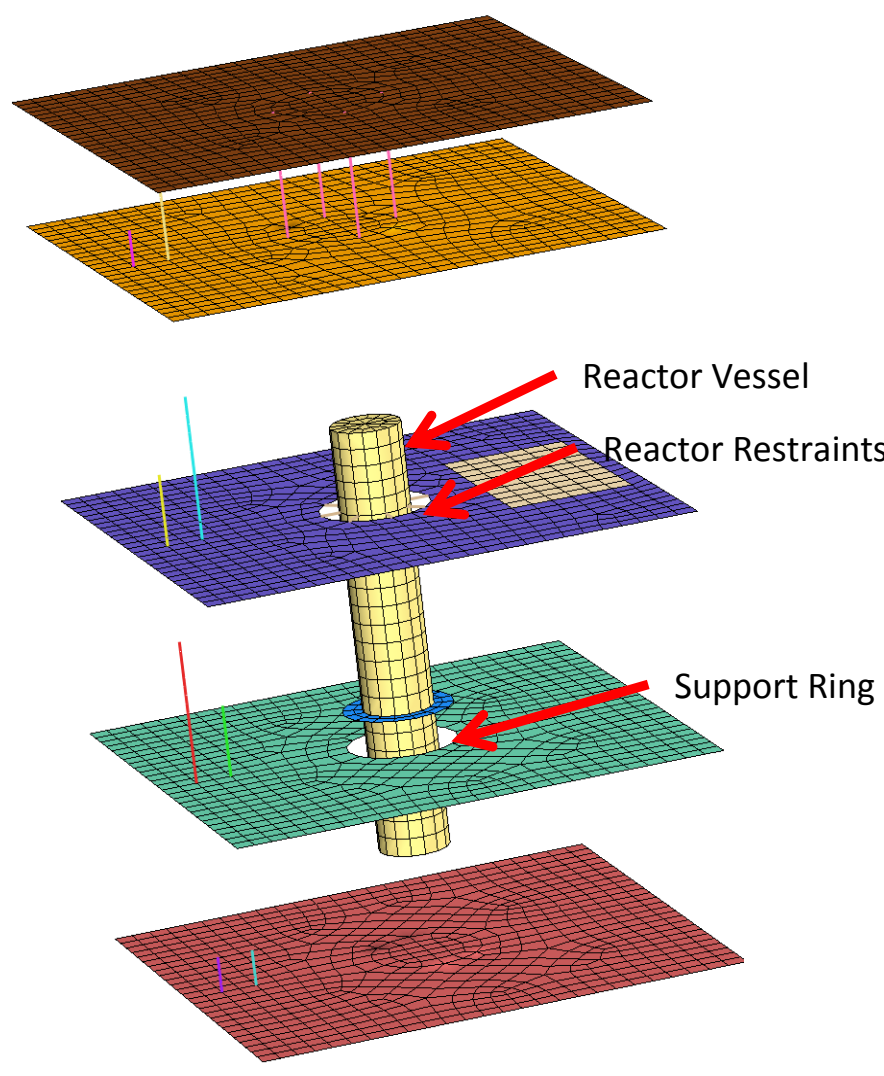

Figure 22 - Reactor structure with outer walls and upper and lower piers removed for clarity

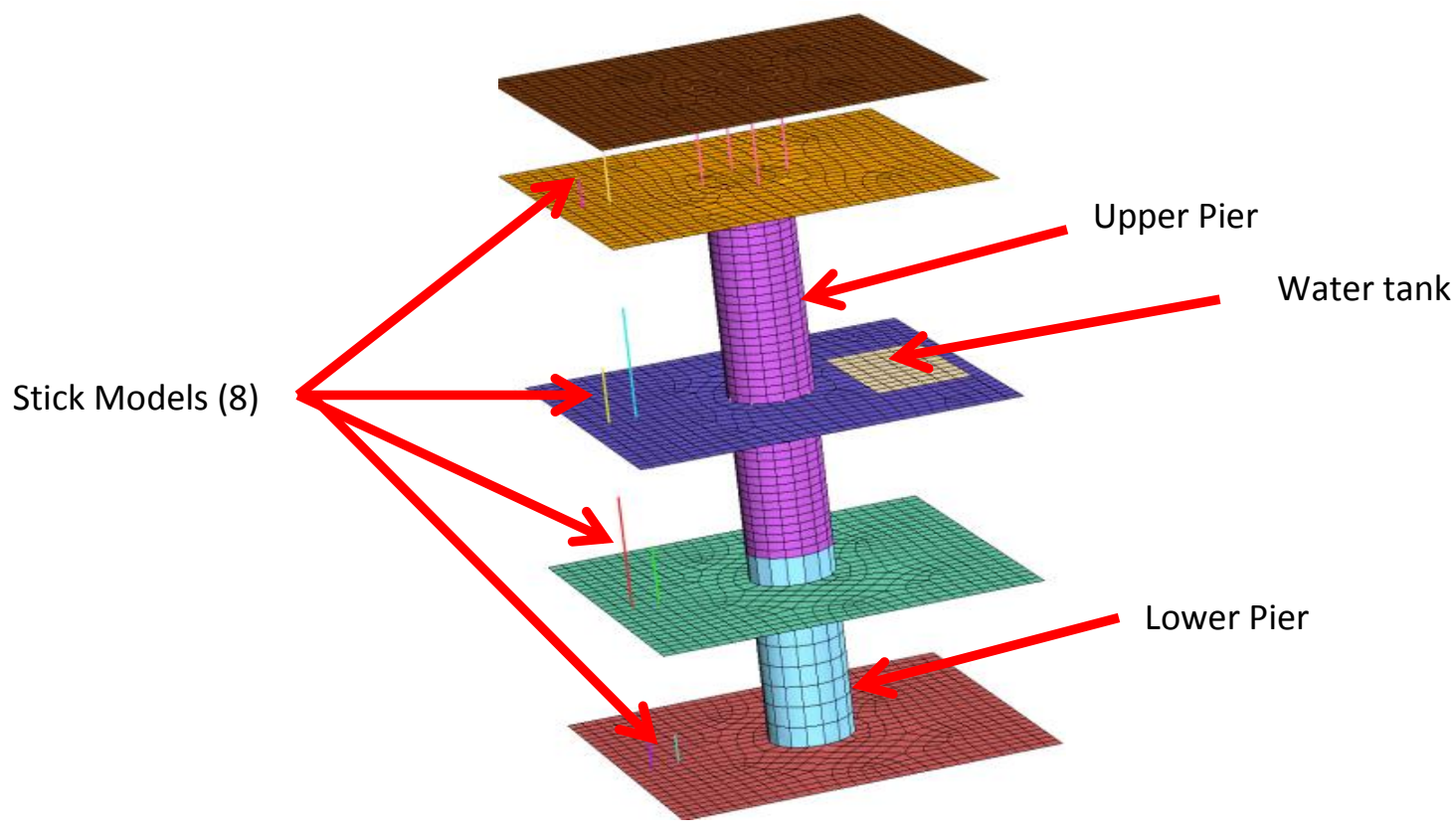

Figure 23 - Reactor structure with outer walls removed for clarity 


\subsubsection{Eigenvalue Analysis of the Reactor Building}

An eigenvalue analysis of the Reactor Building structure was performed assuming a fixed boundary condition at the basemat and ignoring the lateral soil support. This was performed to ascertain whether the resulting structural modes were similar to modes expected for a typical SMR structure. The eigenvalue analysis was performed originally using 3 codes - DIABLO, NIKE3D, and GEMINI. NIKE3D is a serial, solid-mechanics-only code, one of the ancestors of DIABLO. GEMINI is a serial code, developed based on NIKE3D and SAP (Structural Analysis Program), a program developed at University of California, Berkeley for linear structural analysis. Small but insignificant differences were found among the results from the three codes.

Initial eigenvalue analysis runs were made to validate the model and the choice of parameters. For the purposes of a SASSI-DIABLO comparison, only modes with frequencies less than $30 \mathrm{~Hz}$ were considered. These runs were made to ascertain the quality of the structural representation, before transmitting the CUBIT finite element model of the Reactor Building to BNL for SASSI runs. A final eigenvalue analysis was performed with DIABLO, the results of which were confirmed by comparing with those from NIKE3D. Seventy-two (72) modes were found below $30 \mathrm{~Hz}$, thirty of which are listed below. 


\begin{tabular}{|c|c|c|}
\hline Mode\# & Frequency & Description \\
\hline 1 & 0.981 & Stick \#1, mode $1, \mathrm{y}$ \\
\hline 2 & 0.981 & Stick \#1, mode $1, \mathrm{x}$ \\
\hline 3 & 4.56 & Structural mode \\
\hline 4 & 4.56 & Structural mode \\
\hline 5 & 4.95 & Stick \#6 \\
\hline 6 & 4.95 & Stick \#6 \\
\hline 7 & 6.18 & Stick \#1, mode 2 \\
\hline 8 & 6.19 & Stick \#1, mode 2 \\
\hline 9 & 6.84 & Stick \#7 \\
\hline 10 & 6.85 & Stick \#7 \\
\hline 11 & 6.88 & Stick \#3 \\
\hline 12 & 6.88 & Stick \#3 \\
\hline 13 & 8.85 & Structural Twist \\
\hline 14 & 12.4 & Structural Bend \\
\hline 15 & 12.5 & Sticks \#4 and 5 \\
\hline 16 & 12.8 & Structural Z-mode \\
\hline 17 & 13.3 & Stick \#4 \\
\hline 18 & 13.4 & Stick \#4 \\
\hline 19 & 13.8 & Stick \#5 \\
\hline 20 & 13.9 & Stick \#5 \\
\hline 21 & 15.9 & Reactor Vessel Bending \\
\hline 22 & 16.0 & Reactor Vessel Bending \\
\hline 23 & 16.7 & Structural Z mode \\
\hline 24 & 17.0 & Reactor Vessel Bending + Floor 2 \\
\hline 25 & 17.5 & Roof and Floor 3 Bending \\
\hline 26 & 17.6 & Roof and Floor 3 Bending \\
\hline 27 & 18.4 & Roof and Floor 3 Bending \\
\hline 28 & 18.4 & Roof and Floor 3 Bending \\
\hline 29 & 18.4 & General Floor and Vessel Bending \\
\hline 30 & 18.8 & General Floor and Vessel Bending \\
\hline
\end{tabular}

Table 1 - First 30 eigenvalues

\subsubsection{Soil Profile}

The soil profile was developed based on an existing site response analysis of a DOE deep soil site at the Savannah River National Laboratory [31]. However, the existing site response analysis was performed using the actual bedrock depth of more than 1000 feet below the surface. For the current study, to facilitate ease of comparison between a finite element model and SASSI, it was assumed that the bedrock was closer to the surface. This modified site response analysis was performed by Professor Carl Costantino as part of his subcontract with LBNL, using the one-dimensional wave propagation computer code CARES [18] [19], which is similar to the computer program SHAKE [32], [5]. Both are widely used in the nuclear industry for site response analysis. 
The results of this analysis provided the dynamic properties of the soil layers for use in the current study. The layer descriptions and key soil characteristics are provided in Table 2. For the purposes of computing a bulk soil modulus, a constant Poisson's ratio of 0.35 was used, consistent with the type of silty sands typical of the selected DOE site ${ }^{5}$.

\begin{tabular}{|c|l|c|c|c|c|}
\hline Layer & \multicolumn{1}{|c|}{ Name } & $\begin{array}{c}\text { Depth at } \\
\text { the top of } \\
\text { the layer } \\
(\mathbf{f t})\end{array}$ & $\begin{array}{c}\text { Unit Weight } \\
\mathbf{( 1 0 0 0} \\
\text { pounds / } \\
\text { cubic foot) }\end{array}$ & $\begin{array}{c}\text { Effective } \\
\text { overburden } \\
(\mathbf{1 0 0 0} \text { pounds / } \\
\text { square foot) }\end{array}$ & $\begin{array}{c}\text { Initial Shear } \\
\text { Wave } \\
\text { Velocity } \\
\text { (feet/second) }\end{array}$ \\
\hline 1 & Upland & 0 & 0.125 & 1.872 & 1580 \\
\hline 2 & Tobacco Road & 30 & 0.128 & 6.558 & 1240 \\
\hline 3 & Dry Branch & 74 & 0.125 & 12.429 & 1200 \\
\hline 4 & Santee & 123 & 0.123 & 19.607 & 1564 \\
\hline 5 & Warley Hill & 190 & 0.132 & 24.716 & 2153 \\
\hline 6 & Congaree & 205 & 0.132 & 28.805 & 1720 \\
\hline 7 & Four Mile & 252 & 0.126 & 33.545 & 1415 \\
\hline 8 & Snapp & 278 & 0.132 & 36.83 & 1675 \\
\hline 9 & Sawdust Landing & 303 & 0.131 & 42.864 & 2015 \\
\hline 10 & Hard Rock & 370 & 0.15 & N/A & 11,000 \\
\hline
\end{tabular}

Table 2 - Soil Layers

For the purposes of the comparison between SASSI and DIABLO ${ }^{6}$, the lowest layer, the Hard Rock Interface, has been modeled as an infinitely stiff boundary. This is acceptable since the shear wave velocity is about one order of magnitude larger than that of the other soil layers, but without an appreciable difference in density - implying a difference in modulus of a factor of 100.

Using a typical industry approach, in Professor Costantino's site response analysis, the soil layers were characterized according to the EPRI-93 protocol [4] in terms of shear modulus degradation and hysteretic damping (as a percentage of critical damping), both as a function of reference strain values. The shear modulus degradation values are given in Table 3, and illustrated in Figure 24. The hysteretic damping values, as a percentage of critical damping, are given in Table 4 and illustrated in Figure 25.

${ }^{5}$ Carl Costantino, private communication

6 If the Hard Rock Interface is not modeled as an infinitely stiff base, both DIABLO and SASSI require the use of approximate absorbing boundary conditions at the base. As the approximations in the two codes are different, assuming an infinitely stiff base ensures that differences in the codes are not the result of differences in absorbing boundary conditions at the base. 


\begin{tabular}{|c|c|c|c|c|c|c|c|c|c|}
\hline STRAIN (\%) & $\begin{array}{c}\text { Layer } \\
\# 1\end{array}$ & $\begin{array}{c}\text { Layer } \\
\# 2\end{array}$ & $\begin{array}{c}\text { Layer } \\
\# 3\end{array}$ & $\begin{array}{c}\text { Layer } \\
\# 4\end{array}$ & $\begin{array}{c}\text { Layer } \\
\# 5\end{array}$ & $\begin{array}{c}\text { Layer } \\
\# 6\end{array}$ & $\begin{array}{c}\text { Layer } \\
\# 7\end{array}$ & $\begin{array}{c}\text { Layer } \\
\# 8\end{array}$ & $\begin{array}{c}\text { Layer } \\
\# 9\end{array}$ \\
\hline 0.0001 & 1 & 1 & 1 & 1 & 1 & 1 & 1 & 1 & 1 \\
\hline 0.000316 & 1 & 1 & 1 & 1 & 1 & 1 & 1 & 1 & 1 \\
\hline 0.001 & 0.979 & 0.995 & 0.995 & 0.998 & 0.998 & 0.998 & 1 & 1 & 1 \\
\hline 0.00316 & 0.903 & 0.951 & 0.951 & 0.972 & 0.972 & 0.972 & 0.98 & 0.98 & 0.98 \\
\hline 0.01 & 0.734 & 0.867 & 0.867 & 0.899 & 0.899 & 0.899 & 0.923 & 0.923 & 0.923 \\
\hline 0.0316 & 0.488 & 0.665 & 0.665 & 0.726 & 0.726 & 0.726 & 0.774 & 0.774 & 0.774 \\
\hline 0.1 & 0.266 & 0.427 & 0.427 & 0.492 & 0.492 & 0.492 & 0.557 & 0.557 & 0.557 \\
\hline 0.316 & 0.113 & 0.21 & 0.21 & 0.266 & 0.266 & 0.266 & 0.315 & 0.315 & 0.315 \\
\hline 1 & 0.044 & 0.089 & 0.089 & 0.117 & 0.117 & 0.117 & 0.153 & 0.153 & 0.153 \\
\hline 3.16 & 0.044 & 0.089 & 0.089 & 0.117 & 0.117 & 0.117 & 0.153 & 0.153 & 0.153 \\
\hline 10 & 0.044 & 0.089 & 0.089 & 0.117 & 0.117 & 0.117 & 0.153 & 0.153 & 0.153 \\
\hline
\end{tabular}

Table 3 - Soil Layers, EPRI-93 Shear Modulus Degradation Data

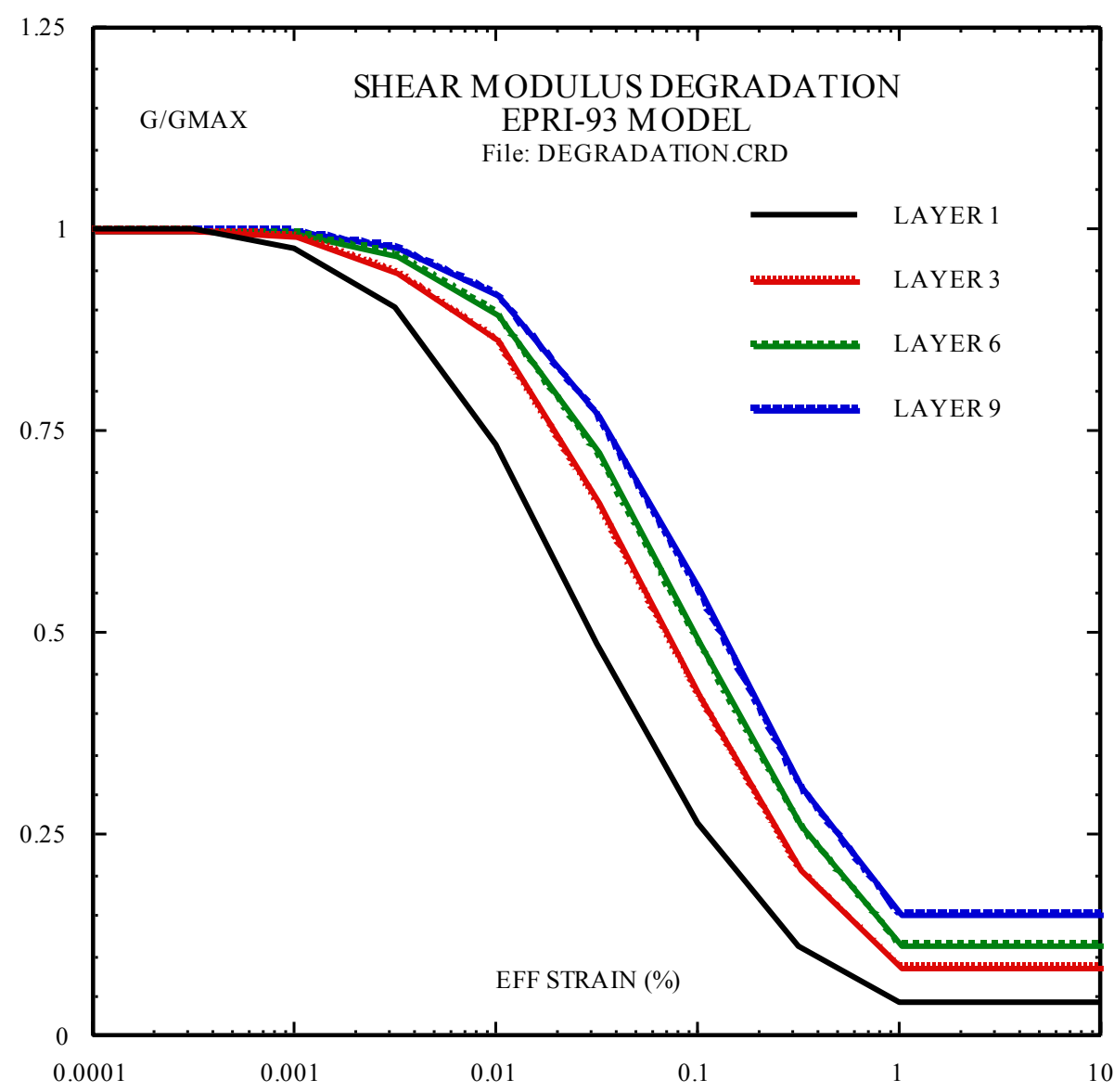

Figure 24 - Soil Layers, EPRI-93 Shear Modulus Degradation, Illustrated 


\begin{tabular}{|c|c|c|c|c|c|c|c|c|c|}
\hline $\begin{array}{c}\text { STRAIN } \\
\text { (\%) }\end{array}$ & $\begin{array}{c}\text { Layer } \\
\# 1\end{array}$ & $\begin{array}{c}\text { Layer } \\
\# 2\end{array}$ & $\begin{array}{c}\text { Layer } \\
\# 3\end{array}$ & $\begin{array}{c}\text { Layer } \\
\# 4\end{array}$ & $\begin{array}{c}\text { Layer } \\
\# 5\end{array}$ & $\begin{array}{c}\text { Layer } \\
\# 6\end{array}$ & $\begin{array}{c}\text { Layer } \\
\# 7\end{array}$ & $\begin{array}{c}\text { Layer } \\
\# 8\end{array}$ & $\begin{array}{c}\text { Layer } \\
\# 9\end{array}$ \\
\hline 0.0001 & 1.43 & 1.15 & 1.15 & 0.95 & 0.95 & 0.95 & 0.85 & 0.85 & 0.85 \\
\hline 0.000316 & 1.43 & 1.15 & 1.15 & 0.95 & 0.95 & 0.95 & 0.85 & 0.85 & 0.85 \\
\hline 0.001 & 1.84 & 1.22 & 1.22 & 1.02 & 1.02 & 1.02 & 0.9 & 0.9 & 0.9 \\
\hline 0.00316 & 2.76 & 1.63 & 1.63 & 1.33 & 1.33 & 1.33 & 1.02 & 1.02 & 1.02 \\
\hline 0.01 & 5.1 & 2.86 & 2.86 & 2.24 & 2.24 & 2.24 & 1.84 & 1.84 & 1.84 \\
\hline 0.0316 & 9.39 & 5.51 & 5.51 & 4.49 & 4.49 & 4.49 & 3.57 & 3.57 & 3.57 \\
\hline 0.1 & 15.51 & 10.41 & 10.41 & 8.67 & 8.67 & 8.67 & 7.14 & 7.14 & 7.14 \\
\hline 0.316 & 22.25 & 17.04 & 17.04 & 15.1 & 15.1 & 15.1 & 13.27 & 13.27 & 13.27 \\
\hline 1 & 27.55 & 22.86 & 22.86 & 21.12 & 21.12 & 21.12 & 19.39 & 19.39 & 19.39 \\
\hline 3.16 & 27.55 & 22.86 & 22.86 & 21.12 & 21.12 & 21.12 & 19.39 & 19.39 & 19.39 \\
\hline 10 & 27.55 & 22.86 & 22.86 & 21.12 & 21.12 & 21.12 & 19.39 & 19.39 & 19.39 \\
\hline
\end{tabular}

Table 4 - Soil Layers, EPRI-93 Hysteretic Damping Data

HYSTERETIC DAMPING RATIO

EPRI-93 MODEL

File: DEGRADATION.CRD

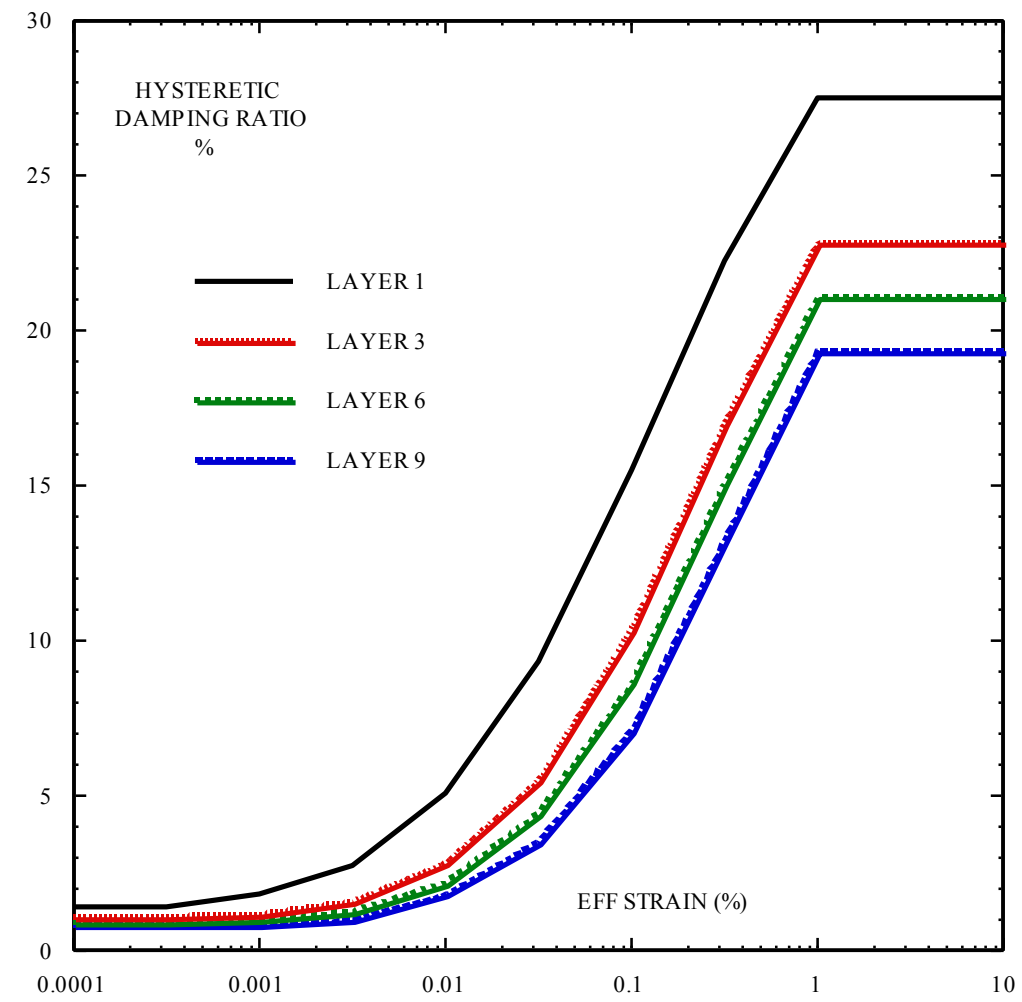

Figure 25 - Soil Layers, EPRI-93 Hysteretic Damping, Illustrated 
In the DIABLO model, the soil layers were modeled using a fully 3D finite element mesh. Including the structure, the model consisted of 467,140 nodes, 444,478 brick elements, 132 beam elements, and 9542 shell elements. A cross-section of this model is provided in Figure 26. The entire soil model was $250 \mathrm{~m}$ deep $\times 250 \mathrm{~m}$ wide $\times 370 \mathrm{ft}$ deep. The soil mesh was divided into 9 layers corresponding to the 9 soil layers of Table 2, and three regions corresponding to the different Bielak regions (see Figure 1). The interior region was a box extending to the base of the structure, with sides $100 \mathrm{~m}$ deep $\times 100 \mathrm{~m}$ wide. The intermediate region extended, as a hollow rectangular extrusion, between $50 \mathrm{~m}$ and $60 \mathrm{~m}$ in both (positive and negative) $X$ and (positive and negative) $Y$ directions. The external region extended out beyond this to $125 \mathrm{~m}$ in both (positive and negative) $\mathrm{X}$ and (positive and negative) $\mathrm{Y}$ directions. The base of the interior region coincided with the base of the soil model, at -370 feet (-112.7758 meters).

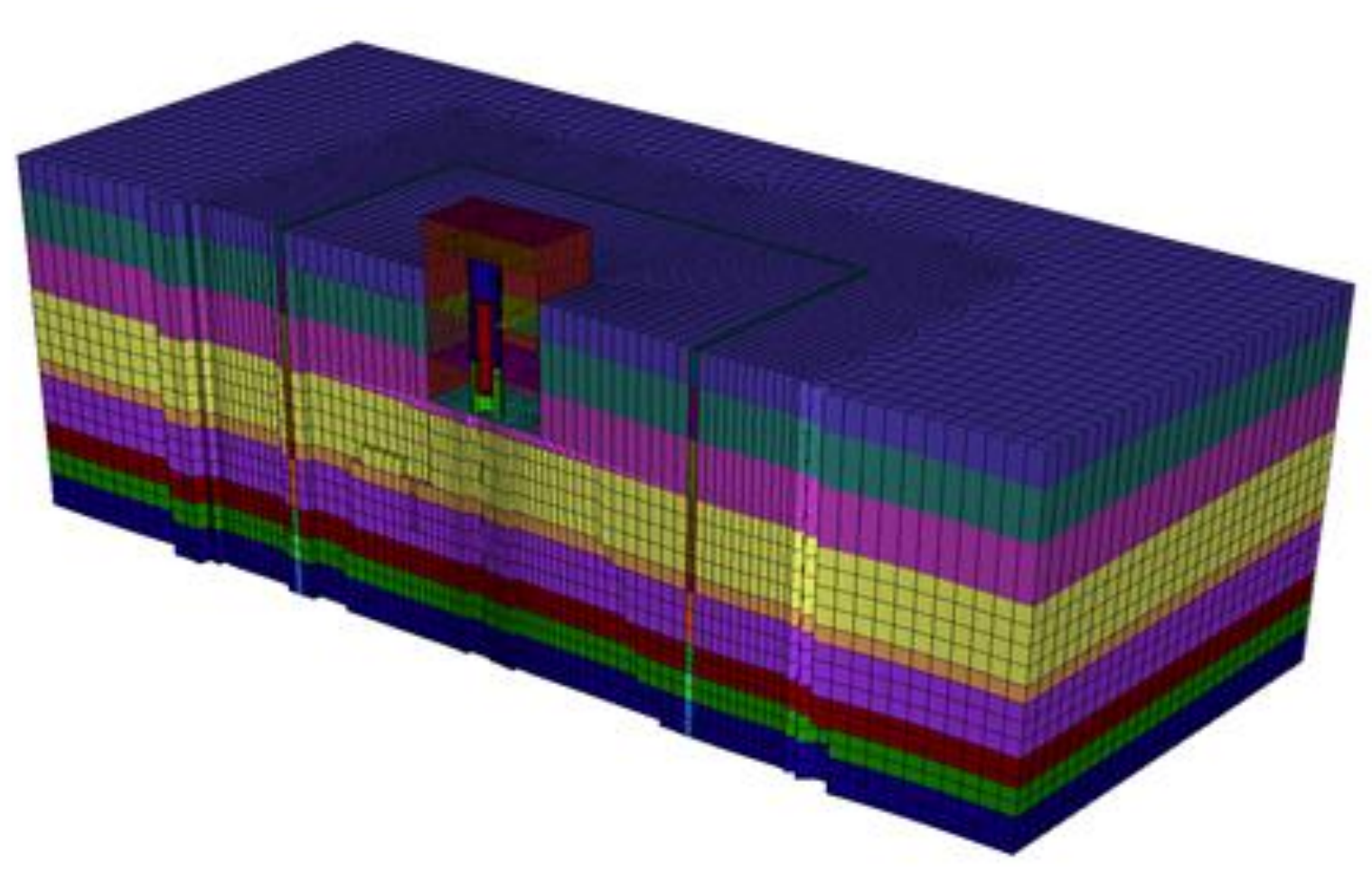

Figure 26 - Reactor model embedded in soil, with a jagged cut at approximately the $Y=0$ plane. The $Z=0$ plane corresponds to the top ground surface.

The horizontal mesh density varied as shown in the picture. In the top three soil layers, the vertical mesh in the soil matched the reactor mesh, which is shown in Figure 21. For layers below the reactor (with the layers numbered from bottom to top), the vertical mesh density was

- Layer 1 (dark blue) - 2 elements through the thickness

- Layer 2 (light green) - 2 elements through the thickness

- Layer 3 (red) -3 elements through the thickness

- Layer 4 (light purple) - 3 elements through the thickness

- Layer 5 (orange) - 3 elements through the thickness

- Layer 6 (yellow) - 4 elements through the thickness 
- Layer 7 (light purple) - 13 elements through the thickness (some below the reactor)

\subsection{Development of Finite Element Technology for SSI analysis}

\subsubsection{A Description of the Time- and Frequency-Domain Finite Element Methods}

DIABLO employs a time-domain finite element methodology. The basic method, [33] is based on the Bubnov-Galerkin method. The equations of motion, with $\rho$ the density, $\ddot{\mathbf{u}}$ the second time derivative of the displacement (e.g. the acceleration), $\boldsymbol{\sigma}$ the stress tensor, and $\mathbf{b}$ the distributed body force (per unit mass), are

\section{Equation 1 - General equations of motion}

$$
\rho \ddot{\mathbf{u}}=\nabla \bullet \boldsymbol{\sigma}+\rho \mathbf{b}
$$

This equation is multiplied by a test function $\mathbf{W}$ and integrated by parts over the volume $\Omega$ (with boundary $\partial \Omega$ ), providing the weighted-residual form

$$
\begin{gathered}
\text { Equation 2 - Weighted-residual form } \\
\int_{\Omega} \rho \ddot{\mathbf{u}}+\boldsymbol{\sigma} \bullet \nabla^{S} \mathbf{w} d \Omega=\int_{\Omega} \rho \mathbf{b} \bullet \mathbf{w} d \Omega+\int_{\partial \Omega}(\boldsymbol{\sigma} \bullet \mathbf{n}) \bullet \mathbf{w} d \Gamma
\end{gathered}
$$

Via standard procedures of displacement-based finite-element analysis [33], this equation leads to a (possibly non-linear) matrix equation relating the second time derivative of the global displacement vector $[\mathrm{U}]$ to the internal force vector $[\mathrm{F}]$ and the external applied load vector $[\mathrm{P}]$

\section{Equation 3 - Discrete equation of motion for the general case}

$$
[M][\ddot{u}]-[F]=[P]
$$

If the material is linearly visco-elastic ${ }^{7}$, then the stress is proportional to the symmetric strain gradient and its time derivative, with the tensor of elasticity coefficients $\mathbf{D}_{e}$ and viscosity coefficients $\mathbf{D}_{d}$

$$
\begin{aligned}
& \text { Equation } 4 \text { - Linear visco-elastic material } \\
& \qquad \begin{aligned}
\boldsymbol{\sigma} & =\boldsymbol{\sigma}\left(\nabla^{s} \mathbf{u}, \nabla^{s} \dot{\mathbf{u}}\right) \\
& =\mathbf{D}_{e} \nabla^{s} \mathbf{u}+\mathbf{D}_{d} \nabla^{s} \dot{\mathbf{u}}
\end{aligned}
\end{aligned}
$$

With this assumption, the internal force vector [F] can be written as a linear function of the global displacement and velocity vectors

\footnotetext{
${ }^{7}$ Kelvin-Voigt linear visco-elasticity assumed. Other possibilities are discussed in Section 3.2.3
} 
Equation 5 - Force vector and resulting stiffness and damping matrices for the linear visco-elastic case

$$
\begin{aligned}
{[F] } & =-\int_{\Omega}\left(\boldsymbol{\sigma} \bullet \nabla^{s} \mathbf{w}\right) d \Omega \\
& =-[K][u]-[C][\dot{u}]
\end{aligned}
$$

Applying this result to Equation 3, the familiar linear finite element matrix equation results

Equation 6 - Dynamic equations of motion for the linear case

$$
[M][\ddot{u}]+[C][\dot{u}]+[K][u]=[P]
$$

In a frequency-domain method such as SASSI, the solution [u] is expanded in terms of Fourier coefficients, i.e.

\section{Equation 7 - Displacements expanded in Fourier coefficients}

$$
[u]=\sum_{i}[A]_{i} e^{i \omega_{i} t}
$$

Then, for each frequency $\omega_{i}$ of interest in the analysis, a load (possibly complex) load vector $[P]_{i}$ is calculated, and the following equation is solved for the displacement coefficients $[A]_{i}$

\section{Equation 8 - Frequency-domain equation}

$$
\left([K]+i \omega_{i}[C]-\omega_{i}^{2}[M]\right)[A]_{i}=[P]_{n}
$$

The term in parentheses which multiplies $[A]_{i}$ is the frequency-dependent "impedance matrix" $X\left(\omega_{i}\right)$, that is

\section{Equation 9 - Frequency-dependent impedance matrix}

$$
X\left(\omega_{i}\right)=\left([K]+i \omega_{i}[C]-\omega_{i}^{2}[M]\right)
$$

As seen in Equation 9, if damping is present (see Section 3.2.3), the impedance matrix has complex coefficients. In standard frequency-domain analysis, Equation 8 is solved for a finite number of frequencies (in SASSI analyses, typically < 100), covering the frequency range of interest. An interpolation method is used to determine the response at other points in frequency-space.

In many frequency-domain codes (including SASSI), it is assumed that the damping matrix is frequencyindependent, or "hysteretic". For hysteretic damping, Equation 9 is replaced by the frequencyindependent version 
Equation 10 - Impedance matrix with frequency-independent ("hysteretic") damping

$$
\bar{X}\left(\omega_{i}\right)=\left([K]+i[\bar{C}]-\omega_{i}^{2}[M]\right)
$$

Then Equation 8 is replaced by

\section{Equation 11 - Frequency-domain equation with frequency-independent damping}

$$
\bar{X}\left(\omega_{i}\right)[A]_{i}=[P]_{n}
$$

In contrast to the frequency-domain approach, in a time-domain code such as DIABLO, the time interval of the analysis is subdivided into a discrete number of "time steps". The motion within the time step is prescribed according to a "time integration" algorithm. The Newmark [34] method is typically used in such analyses, because it is second order accurate in time, and is for linear elastic materials is energyconserving with the appropriate choice of parameters. The Newmark method is a two-parameter $(\alpha, \beta)$ family of time integration methods.

\section{Equation 12 - Newmark time integration}

$$
\begin{aligned}
& u_{n+1}=u_{n}+\dot{u}_{n} \Delta t+(1 / 2-\beta) \Delta t^{2} \ddot{u}_{n}+\beta \Delta t^{2} \ddot{u}_{n+1} \\
& \dot{u}_{n+1}=\dot{u}_{n}+(1-\alpha) \Delta t \ddot{u}_{n}+\alpha \Delta t \ddot{u}_{n+1}
\end{aligned}
$$

All simulations using DIABLO have been performed with the standard second-order accurate, energyconserving set parameters $\alpha=1 / 2, \beta=1 / 4$. Since the displacement and velocity at the beginning of the step are known, Equation 12 defines the displacement $u_{n+1}$ and velocity $\dot{u}_{n+1}$ at the end of the step entirely in terms of the unknown acceleration at the end of the step, $\ddot{u}_{n+1}$. That is, one has $u_{n+1}\left(\ddot{u}_{n+1}\right)$, and $\dot{u}_{n+1}\left(\ddot{u}_{n+1}\right)$. For a given time step size $\Delta t$, let $t_{n+1}=t_{n}+\Delta t$. In the linear case, utilizing Equation 12 in Equation 6 provides

Equation 13 - Linear discrete time stepping

$$
\left([M]+\alpha \Delta t[C]+\beta \Delta t^{2}[K]\right)\left[a_{n+1}\right]=[P]-[C]\left(\left[\dot{u}_{n}\right]+(1-\alpha) \Delta t\left[\ddot{u}_{n}\right]\right)-[K]\left(\left[u_{n}\right]+\Delta t\left[\dot{u}_{n}\right]+(1 / 2-\beta) \Delta t^{2}\left[\ddot{u}_{n}\right]\right)
$$

Similar to the impedance matrix of Equation 9, for fixed time step, a dynamic stiffness matrix $G$ is formed

\section{Equation 14 - Linear dynamic stiffness matrix}

$$
G(\Delta t)=\left([M]+\alpha \Delta t[C]+\beta \Delta t^{2}[K]\right)
$$

In contrast to the frequency-domain approach, a single solution in the time-domain will resolve all frequencies up to the resolution of the mesh and the time step selected. Of course, this requires 
solution of Equation 13 (or the equivalent equation in the nonlinear case) numerous times, one for each time step. Given a maximum frequency of interest $f_{\max }$, the time step should be chosen such that [35]

\section{Equation 15 - Time step limitation}

$$
\Delta t \leq \frac{1}{10 f_{\max }}
$$

Lysmer [36] reports that the maximum mesh size is dependent both on the shear wave speed $V_{s}$ and the maximum frequency of interest, $f_{\max }$. In addition, the maximum mesh size is dependent on whether a "lumped" or "consistent" mass matrix is used. Interestingly, the best results are provided when the mass matrix is " $50 \%$ lumped", meaning that $1 / 2$ of the theoretical "consistent" mass matrix is used, and combined with $1 / 2$ of the standard lumped mass matrix. The required mesh density values reported by Lysmer are

Equation 16 - Mesh size limitation

$$
\begin{aligned}
& h_{0 \% \text { lumped }} \leq \frac{V_{s}}{8 f_{\max }} \\
& h_{50 \% \text { lumped }} \leq \frac{V_{s}}{5 f_{\max }} \\
& h_{100 \% \text { lumped }} \leq \frac{V_{s}}{8 f_{\max }}
\end{aligned}
$$

For Diablo calculations, the mass matrix used was $100 \%$ lumped.

In contrast to the frequency-domain method of SASSI, a difficulty in time-domain analysis is the application of proper boundary conditions, since the time-domain method cannot take advantage of the frequency-domain solution of Waas [22]. Discussion of the implementation of satisfactory boundary conditions is covered in Section 3.2.4.

\subsubsection{Stiffness (Moduli) representation in SASSI versus Nonlinear Time Domain Analyses}

One of the key features of a SASSI analysis is the treatment of the nonlinearities inherent in soil motion using the "equivalent-linear" approach. The frequency-domain method relies inherently on the principle of superposition, which means Equation 8 can be solved independently [37] for each frequency of interest, and the results superimposed using Equation 7. This superposition is possible only if the material is linear - that is, only if the material response is independent of the magnitude. However, as Figure 24 and Figure 25 illustrate, for real soils this is not the case even for relatively small strain levels (e.g. below $0.001 \%$ strain for Soil Layer 1 ).

To compensate for the strain-dependence of the properties, SASSI recommends that "equivalent linear" soil properties be used. A computer program such as SHAKE [32], [5] or CARES [18], [19] is used to determine these properties. This process is illustrated by Figure 27. SHAKE (or CARES) utilizes the same 
vertically-propagating horizontal shear wave as will be subsequently used to drive the corresponding SASSI analysis. SHAKE (or CARES) calculates the solution to the 1-D wave propagation problem for a set of material properties for each layer consistent with the available shear modulus degradation and damping data, tabulated versus maximum strain (for example Table 3 and Table 4), starting with the tabulated values for zero maximum strain. After each SHAKE (or CARES) analysis, the input modulus and damping values for each soil layer are adjusted consistent with the values tabulated for the maximum strain level that is found to occur in each layer. The 1-D wave propagation problem is then re-analyzed with the new material properties. The process is repeated until the maximum strain levels calculated for each layer do not change. The final modulus and damping values calculated by the SHAKE/CARES process are then used as input to the SASSI analysis. When not available from direct measurement, the shear modulus degradation and damping ratio curves used as inputs by SHAKE or CARES may be estimated based upon available data and empirical relationships [38], [39], [6].

SASSI therefore uses constant (strain-independent) modulus and damping values at a particular material point during the entire duration of the seismic event. During a seismic event, the strain experienced at a material point ranges from zero to some maximum value, and hence the appropriate modulus and damping $^{8}$ at a point in time differs (typically the modulus stiffer and the damping softer) from that utilized by SASSI. Furthermore, the maximum strain calculated by SHAKE/CARES is a prediction - it is quite possible that the maximum strain in the SASSI calculation exceeds the predicted maximum strain. Such a result is not unexpected (in some sense it is the reason SSI calculations are performed in the first place), and often occurs at regions of high stress concentration in the near-field, such as the corners of the basemat.

"Equivalent linear" methods are valid only under very specific assumptions, and these assumptions become less valid as both the depth of the embedment increases (which increases the size and influence of the near-field zone), and as the intensity of the earthquake increases (which increases the range of strains which the material experiences). For SMRs, which will generally be deeply embedded and for which survival under high intensity earthquakes is paramount, both issues are serious. These effects can be particularly significant for large earthquakes with significant high-frequency content, for as Assimaki [8] notes: "since material damping is a function of amplitude, high frequencies associated with small amplitude cycles of vibration have substantially less damping than the predominant frequencies of the excitation, and these components are artificially suppressed when hysteretic damping is taken constant."

\footnotetext{
${ }^{8}$ Typical shear modulus degradation and damping values are typically calculated as average values over a strain cycle. The values that are extracted are therefore not tangent to the stress/strain curve, and are better characterized as secant moduli.
} 


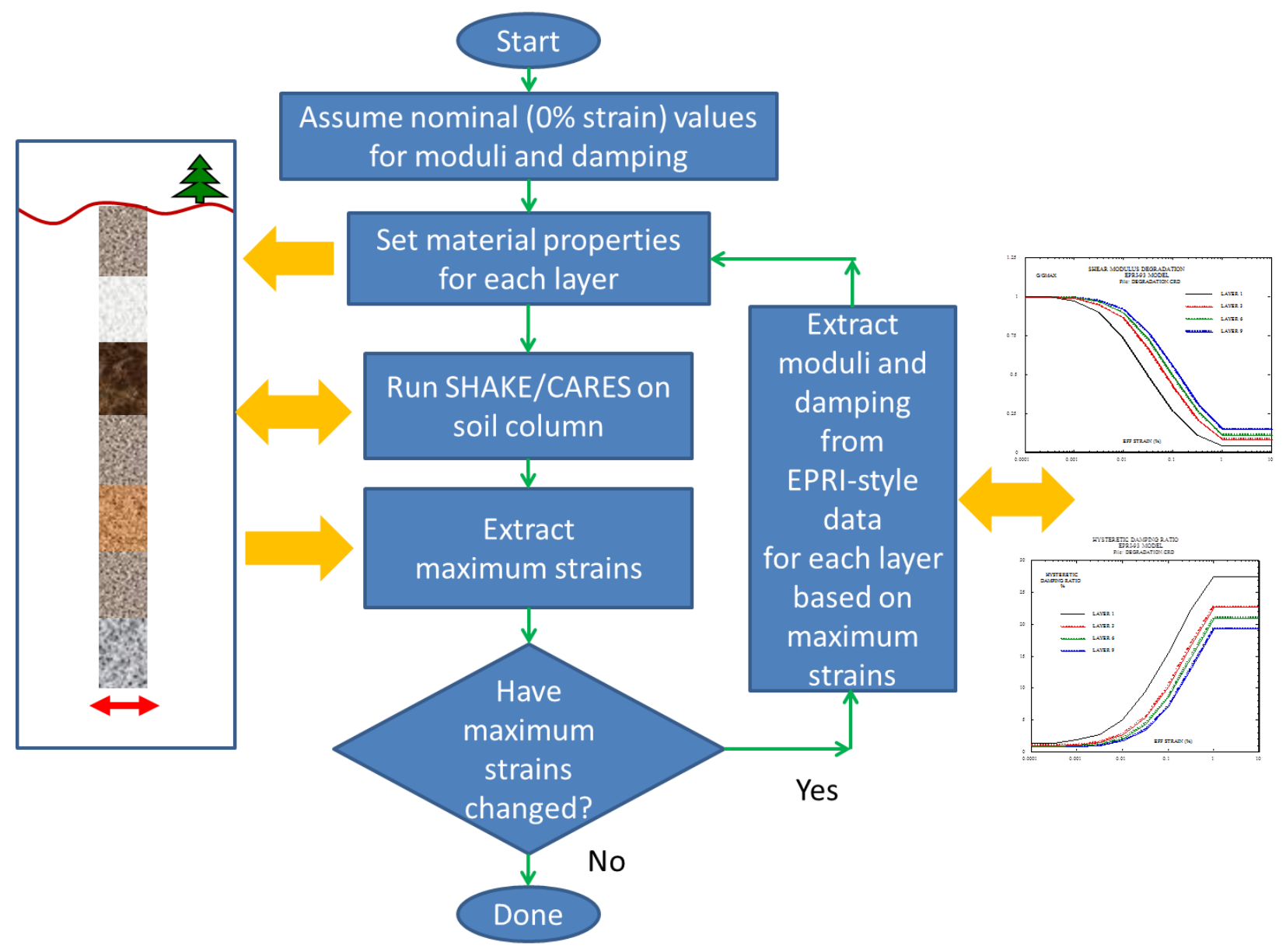

Figure 27 - Calculation of equivalent-linear soil properties using SHAKE (or CARES) assuming a verticallypropagating shear wave corresponding to a particular earthquake

Some recent examples where (mostly for 1-D soil analysis) explicit inclusion of changes in soil response based upon intensity has been accounted for include Phillips [40] and Drosos [14]. When the actual 3-D soil properties are considered, many authors note the significant dependence on confining pressure, pore pressure, and void ratio, all of which can change drastically in the presence of reactor rocking motions, see for example Zefra [10], Bonilla [9], Reyes [41], Gellis [42], and the review article by Soga [15], who notes the "experimental finding that volume change upon shearing is associated with the mean pressure change along with shear induced dilatancy". Assimaki [8] notes "Laboratory experimental data on sand samples subjected to high confining pressures, show that for highly confined materials, both the standard shear modulus reduction factor and the damping versus shear strain amplitude overestimate the capacity of the soil to dissipate energy". Soil response is highly dependent on confining pressure, much of which is a function of pore water pressure. Both three-dimensional stress states (as occur during rocking motions of a structure), and the evolution of pore water pressure due to cyclic deformation, can have a great effect on material response. Matasovic [7] notes that "The degradation of fully saturated sands cyclically sheared in undrained conditions takes place due to the pore-water pressure buildup, which causes the reduction of effective stresses and consequently the shear strength and moduli", see Figure 28 


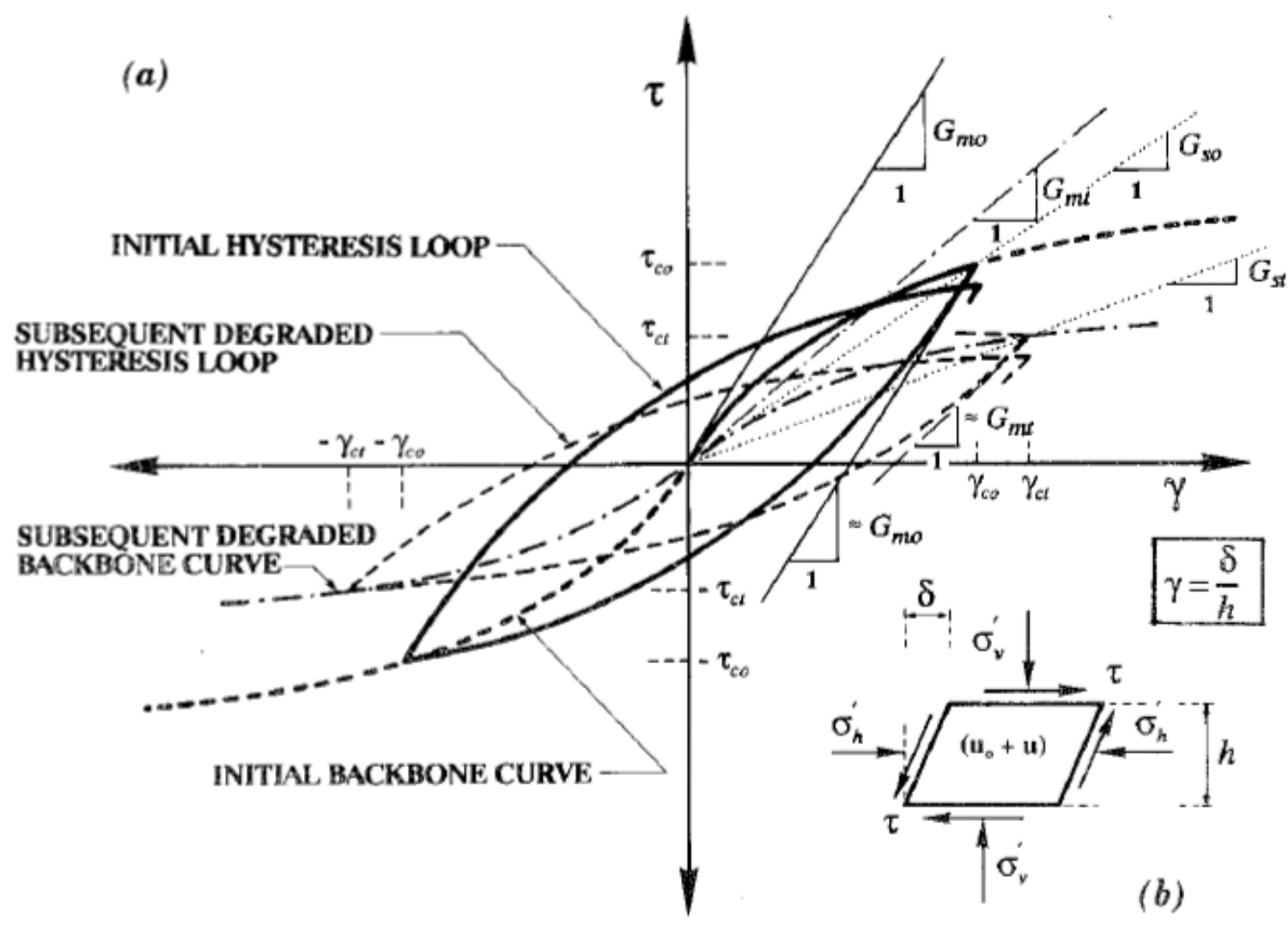

Figure 28 - Hysteresis and Modulus Degradation as a result of cyclic effects in liquefiable sands (Matasovic, 1993)

Restricting the context to 1-D equivalent linear models, it is acknowledged that significant dependence exists on confining pressure. Zhang [6] investigated how shear modulus degradation and damping curves changed as a function of confining pressure. Figure 29 (Figure 15 from [6], illustrates the large differences in curves that result from changes in confining pressure. The fact that confining pressure ranges in the figure over 2 orders of magnitude may indicate that the effect is small, except that the influence of the structure may lead to local unloading of the confining pressure (basemat uplifit). 

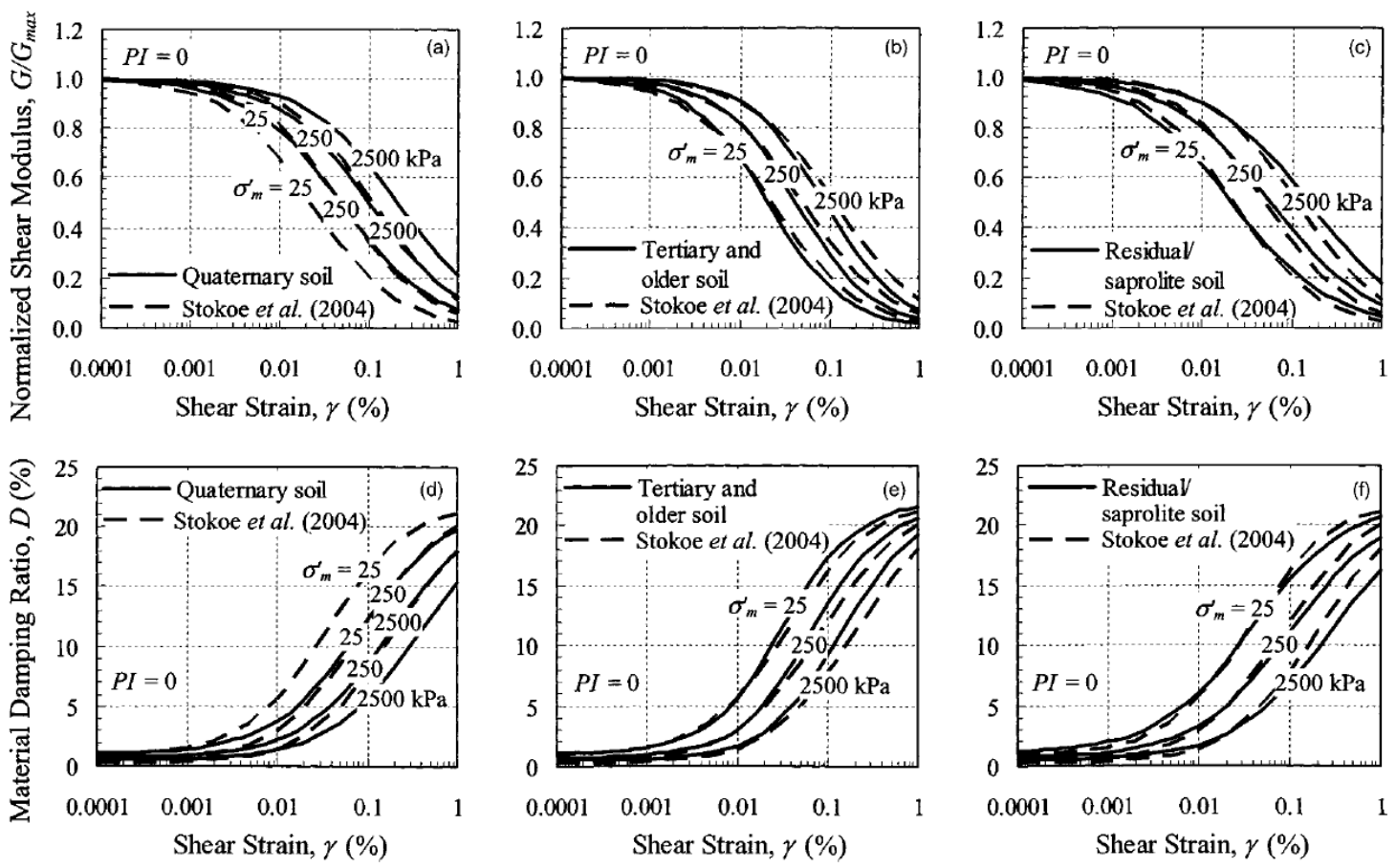

Fig. 15. Comparison of recommended $G / G_{\max }-\log \gamma$ and $D-\log \gamma$ curves with curves by Stokoe et al. (2004) for plasticity index $=0$ soil at $\sigma_{m}^{\prime}=25,250$, and $2500 \mathrm{kPa}$

Figure 29- Shear Modulus Degradation and Damping curves as a function of confining pressure (Zhang 2005)

Ishibashi and Zhang [39], among other authors, present similar results. Wolf [11], in an article otherwise dedicated to delineating the simplest possible means of analysis, emphasizes the nonlinear effects that result from rocking motion (partial uplift of the basemat) in deeply embedded structures.

In summary:

1. It is an established fact that soils, even when subject to simple 1-D shearing motions, experience reduced stiffness (degraded modulus) and increased damping at large strains. Strain-rate dependence also exists but has been less well documented.

2. The equivalent linear method used in SASSI attempts to bypass this fact by estimating the shear modulus and damping based upon maximum soil column motions for a selected earthquake amplitude. This process can be potentially unconservative especially for larger earthquakes, because

a. The impedance is underestimated at high frequencies since constant (large) damping and (reduced) modulus is used even where the displacements are small, such as may occur at high frequencies. This may lead to underestimating the amplification in this area of the phase space.

b. The impedance is overestimated in regions where (locally, due to SSI effects) larger than predicted strains occur, perhaps leading to underprediction of maximum displacements - such as might occur under the basemat edges where large local stresses may occur.

c. The influence of changes in confining pressure, cyclic degradation, and pore pressure effects are completely disregarded. These are especially important in larger earthquakes with long time duration. 
3. Nonlinear analysis in the time domain is a rational method by which such effects can be quantified.

\subsubsection{Representation of Damping (Dissipation) in SASSI versus Nonlinear Time-Domain Analyses}

The developments of the previous sections have emphasized linear elastic analysis, thus Equation 4 after discretization in space leads to Equation 6, and after choice of either frequency domain or discrete time stepping, Equation 8 or Equation 13, respectively. A linear elastic material does not dissipate energy, and yet it is known that soils are highly dissipative. There are various dissipation mechanisms available for representing soils - plasticity, visco-elasticity, visco-plasticity. The standard method for representing dissipation in the frequency domain is by frequency-independent, or "hysteretic" damping, as mentioned in Section 3.2.1, e.g. Equation 10). Unfortunately, hysteretic damping, although convenient mathematically in the frequency domain, is not without its problems in the time domain, and in any case is by no means a complete description of the dissipative response of the material.

Beginning with Equation 6, if the damping matrix satisfies the Caughey criterion [43], the system can be diagonalized via standard modal analysis procedures.

Equation 17 - Caughey criterion for diagonalizable damping matrix

$$
[C][M]^{-1}[K]=[K][M]^{-1}[C]
$$

Given mode $n$ with undamped natural frequency $\omega_{n}$, the characteristic equation for that mode is that of a Single Degree of Freedom (SDOF) oscillator

Equation 18 - Normalized single degree of freedom oscillator

$$
\ddot{u}_{n}+2 \xi_{n} \omega_{n} \dot{u}_{n}+\omega_{n}^{2} u_{n}=f_{n}(t)
$$

The quantity $\xi_{n}$ is the damping ratio. When the damping ratio approaches $\xi_{n}=1$, the system does not oscillate when subjected to a perturbation; that is, the system returns to zero as fast as possible, and the system is termed as "critically damped".

Typically, "Rayleigh" damping is used, where the damping matrix $[C]$ is proportional to the mass ${ }^{9}$ and stiffness matrices ${ }^{10}$. See, for example, [44].

\footnotetext{
${ }^{9}$ Although convenient and commonly used, it should be noted that mass-matrix-proportional damping, $\left(\alpha_{R}>0\right)$, is non-physical - it is the equivalent of the material operating in a kind of "goo" that acts to restrain even rigid-body motion

${ }^{10}$ Note that Rayleigh damping can be imposed locally such that different materials can have different Rayleigh Coefficients.
} 


\section{Equation 19 - Rayleigh damping}

$$
[C]=\alpha_{R}[M]+\beta_{R}[K]
$$

Here, $\alpha_{R}$ and $\beta_{R}$ are the mass- and stiffness- proportional Rayleigh coefficients [43]. Transformed to modal coordinates, this produces the following relationship between the undamped natural frequency $\omega_{n}$ of mode $n$, the damping ratio $\xi_{R, n}$ created by Rayleigh damping, and the Rayleigh coefficients

\section{Equation 20 - Damping ratio for Rayleigh damping as a function of natural frequency}

$$
2 \xi_{R, n}=\frac{1}{\omega_{n}} \alpha_{R}+\omega_{n} \beta_{R}
$$

That is, the damping ratio varies for modes with different natural frequencies. In particular, for $\alpha_{R}>0$, the damping ratio becomes large for low-frequency modes, and for $\beta_{R}>0$, the damping ratio becomes large for high-frequency modes. Extensions to Rayleigh Damping are possible which can provide greater flexibility in the variation of damping ratio with the natural frequency of the mode - see [43] and [45].

When subject to a forced sinusoidal displacement of the form $u=\bar{u} e^{i \omega t}$, the response function for a single-degree-of-freedom oscillator takes the form

\section{Equation 21 - Forced vibration of a damped SDOF oscillator}

$$
f_{n, \omega}(t)=\left(-1+2 i \xi_{n} \frac{\omega_{n}}{\omega}+\left(\frac{\omega_{n}}{\omega}\right)^{2}\right) \omega^{2} \bar{u} e^{i \omega t}
$$

The response function can be split into elastic and dissipation components.

\section{Equation 22 - Elastic and dissipation forces for a forced damped SDOF oscillator}

$$
\begin{aligned}
& f_{n, \omega, \text { elastic }}(t)=\left(-1+\left(\frac{\omega_{n}}{\omega}\right)^{2}\right) \omega^{2} \bar{u} e^{i \omega t}=\left(\omega_{n}^{2}-\omega^{2}\right) \bar{u} e^{i \omega t} \\
& f_{n, \omega, \text { dissipation }}(t)=\left(2 i \xi_{n} \frac{\omega_{n}}{\omega}\right) \omega^{2} \bar{u} e^{i \omega t}=2 i\left(\xi_{n} \omega_{n} \omega\right) \bar{u} e^{i \omega t}
\end{aligned}
$$

For a given displacement magnitude, the dissipation force increases linearly with frequency, whereas the elastic force has a constant component and a component proportional to the square of the frequency. At the natural frequency of the oscillator, the elastic component vanishes, and the forcing function is governed entirely by the dissipation. The dissipation per harmonic cycle takes the form [46], page 1132 ), where $\bar{\omega}$ is the frequency of the forced oscillation, 
Equation 23 - Dissipation of a viscously damped SDOF oscillator subject to forced harmonic displacements

$$
W_{D, V}=2 \pi \bar{u}^{2} \xi_{n} \omega_{n} \omega
$$

Equation 23 is valid irrespective of the value of the damping ratio. Noting that the maximum stored energy for the cycle is $W_{S E \text {, max }}=\frac{\bar{u}^{2} \omega_{n}^{2}}{2}$, one can write

Equation 24 - Ratio of the dissipation per cycle to the maximum stored elastic energy for a viscously damped SDOF oscillator

$$
\frac{W_{D, V}}{W_{S E, \max }}=\frac{2 \pi \bar{u}^{2} \xi_{n} \omega_{n} \omega}{\frac{\bar{u}^{2} \omega_{n}^{2}}{2}}=4 \pi \xi_{n} \frac{\omega}{\omega_{n}}
$$

That is, the dissipation, as a fraction of the elastic stored energy, increases linearly with the frequency of the forced oscillation $\omega$, and inversely with the natural frequency of the mode $\omega_{n}$ - hence even if the critical damping is constant for each mode, the energy dissipation will vary both as a function of frequency and mode number.

Oftentimes, the dissipation is characterized in terms of the loss factor $\eta$, which is the ratio of the dissipation per cycle to the maximum stored energy, scaled by $2 \pi$. The loss factor for the Kelvin-Vogt model is therefore proportional to the driving frequency:

Equation 25 - Loss factor for viscous damping is proportional to driving frequency

$$
\eta_{V}=\frac{W_{D, V}}{2 \pi W_{S E, \max }}=2 \xi_{n} \frac{\omega}{\omega_{n}}
$$

Much of the lack of success of time-domain analyses in the recent past can be attributed to reliance on simplistic representations of damping. An example is the recent work by BNL comparing SASSI approaches to LS-DYNA [47], where Rayleigh damping was used to account for the soil dissipation. LSDYNA results vastly overshot SASSI results in the mid-frequency range, a result that can be attributed almost entirely to the inadequate modeling of damping, see Figure 30 which is a duplicate of Figure 4.3.5-14 from the reference. 


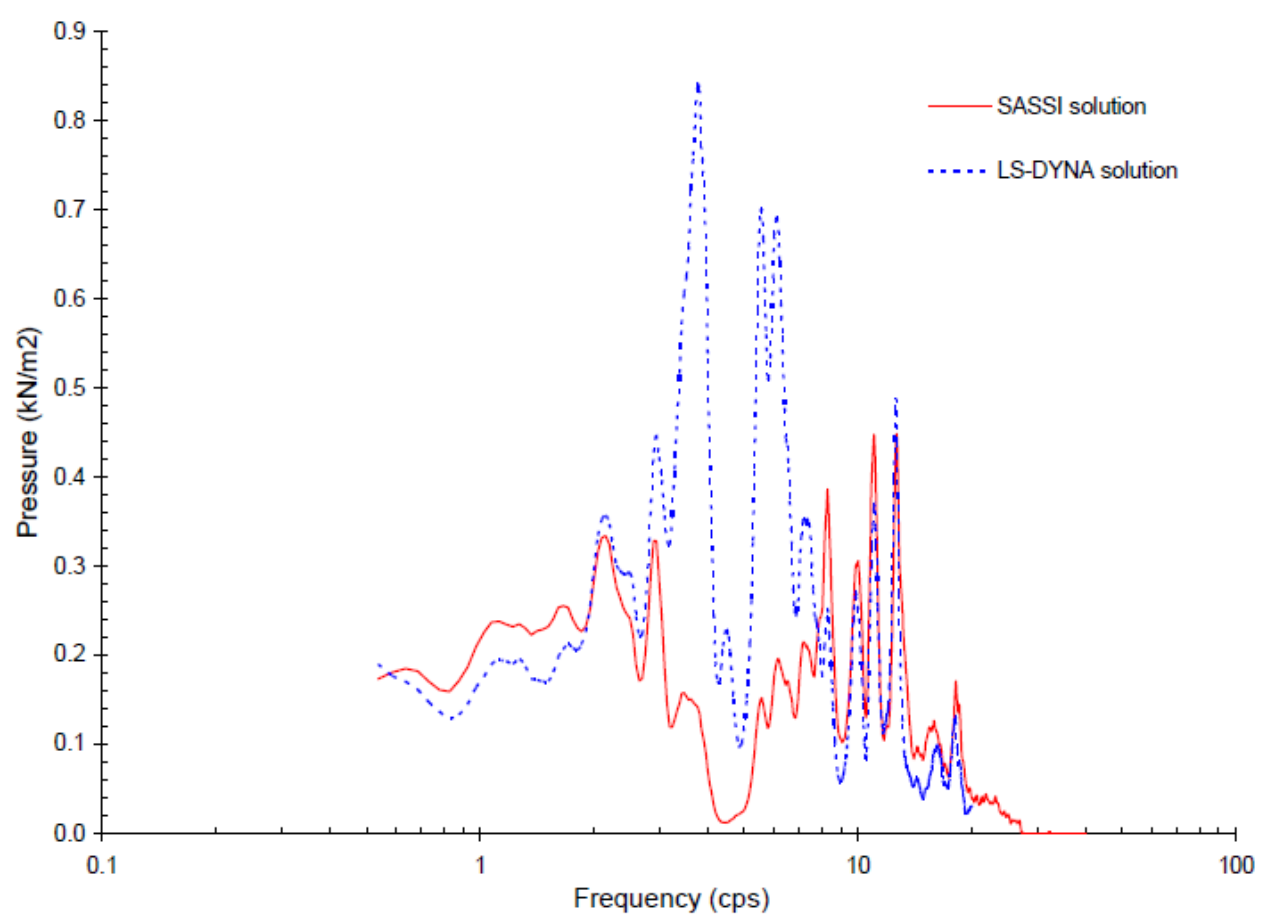

Figure 4.3.5-14 Fourier Spectra of Soil Pressure Computed at the Head-on Soil Element near Mid-Height Embedded Wall for the $46 \mathrm{~m}$ DOB LD-DYNA model.

Figure 30 - LS-DYNA results using Rayleigh damping versus SASSI results (Xu, 2006)

In contrast to this approach, SASSI and SHAKE assume $\mathbf{D}_{d}=\mathbf{0}$ in Equation 4, and assume an elasticity tensor with complex coefficients, that is

Equation 26 - Elasticity tensor for SASSI frequency-domain approach

$$
\mathbf{D}=\mathbf{D}_{e}+i\left[\begin{array}{cccccc}
M_{d} & 2 G_{d} & 2 G_{d} & 0 & 0 & 0 \\
2 G_{d} & M_{d} & 2 G_{d} & 0 & 0 & 0 \\
2 G_{d} & 2 G_{d} & M_{d} & 0 & 0 & 0 \\
0 & 0 & 0 & 2 G_{d} & 0 & 0 \\
0 & 0 & 0 & 0 & 2 G_{d} & 0 \\
0 & 0 & 0 & 0 & 0 & 2 G_{d}
\end{array}\right]
$$

The complex components $M_{d}$ and $G_{d}$ are assumed independent of frequency and proportional to the elastic constants, using a specified fraction of "critical damping" for shear $\left(\xi_{S}\right)$ and plane $\left(\xi_{P}\right)$ waves, respectively. (See also [48], equations 3.8-1a and 3.8-1b) 


\section{Equation 27 - SASSI and SHAKE frequency-independent damping matrix}

$$
\begin{aligned}
G_{d} & =\left(2 \xi_{s}\right) G=\left(2 \xi_{s}\right) \mu \\
M_{d} & =2 \xi_{p} M=2 \xi_{p}(\lambda+2 \mu)
\end{aligned}
$$

Given limited data, typically $\xi_{S}$ and $\xi_{P}$ are typically taken to be equal. The justification for this assumption is given by Schnabel [32] "experiments on many soil materials indicate that $\mathrm{G}$ and $\beta$ are nearly constant over the frequency range of interest". This produces an equation of motion of the form of Equation 11, with complex stiffness (see also Equation 10).

\section{Equation 28 - Complex frequency-independent stiffness with damping}

$$
[K]^{*}=[K]+i[C] \Leftrightarrow \bar{X}(\omega)=[K]^{*}-\omega^{2}[M]
$$

For a single-degree-of-freedom oscillator, the resulting equation takes the form

Equation 29 - Single degree-of-freedom oscillator with complex stiffness (hysteretic damping)

$$
\ddot{u}+\left(2 \xi_{n} i+\omega_{n}^{2}\right) u=f_{n}
$$

Then, the dissipation per cycle for the system of Equation 29, when subject to a harmonic excitation, takes the form (see [46], equation 6)

Equation 30 - Dissipation of a SDOF system with complex stiffness (hysteretic damping)

$$
W_{D, H}=2 \pi \bar{u}^{2} \xi_{n} \omega_{n}^{2}
$$

Then, the loss factor is constant as a function of the driving frequency

Equation 31 - Loss factor for Hysteretic Damping as a Function of Driving Frequency

$$
\eta_{H}=\frac{W_{D, H}}{2 \pi W_{S E, \max }}=2 \xi_{n}
$$


Note that, in contrast to Equation 23 and Equation 24, the dissipation defined by Equation 30 does not increase with frequency. Bert [49] identifies $\mathrm{K}^{*}$ in Equation 28 as the "Kimball-Lovell" stiffness from work by Kimball and Lovell dating back to 1927. As the damping is proportional to the displacement but not the velocity, this kind of damping is also generally termed "hysteretic" as it is generally interpreted as being due to plasticity and/or friction instead of being due to viscous terms. The dissipation is perfectly flat with respect to frequency [50], compared with Maxwell, Kelvin, or Rayleigh damping, see Figure 31. In the figure, Model (a) is a Maxwell model, Model (b) is Kelvin-Vogt, Model (c) is hysteretic damping, and Model (d) is standard linear solid. Note that the standard linear solid has more constant behavior in the central range of frequencies at the cost of non-constant equivalent static stiffness.
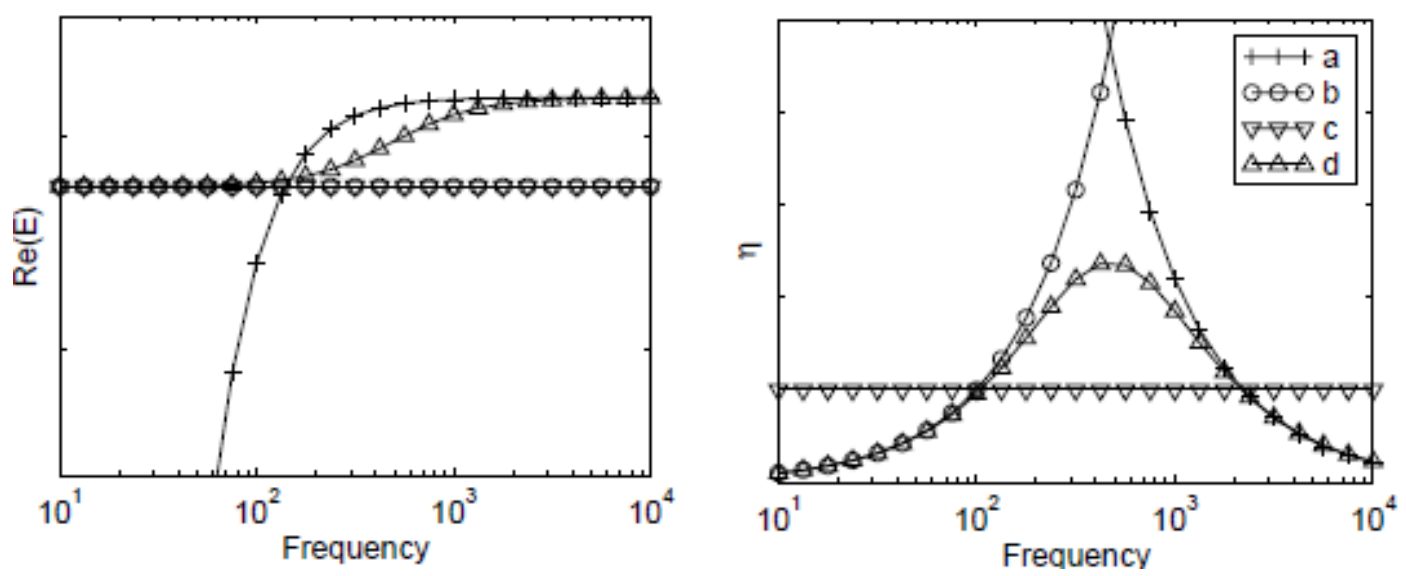

Figure 31 - Comparison of normalized equivalent static stiffness (left) and loss factor (right) for various models, from (Balmes, 2006).

Unfortunately, interpretation of Equation 28 in the time domain is problematic. In fact, Crandall [12] among other authors (see, for example [46] for a comprehensive review) has shown that frequencyindependent damping (even if band-limited [51]) is not causal, and that it leads to spurious results in the time domain. Because frequency-independent damping is not physical, the hysteresis loops so generated do not have the correct shape ( [49], see also Figure 32.

As an alternative to Kelvin-Voigt-type viscous damping in the time domain, one may employ various more complicated (but still linear) material models based upon convolution. One convenient general form of such models is based upon the Volterra equation:

Equation 32 - Generalized linear viscoelasticity using the Volterra equation

$$
\boldsymbol{\sigma}(t)=\int_{0}^{t} \mathbf{G}_{R}(t-\tau) \bullet \nabla^{s} \dot{\mathbf{u}}(\tau) d \tau
$$




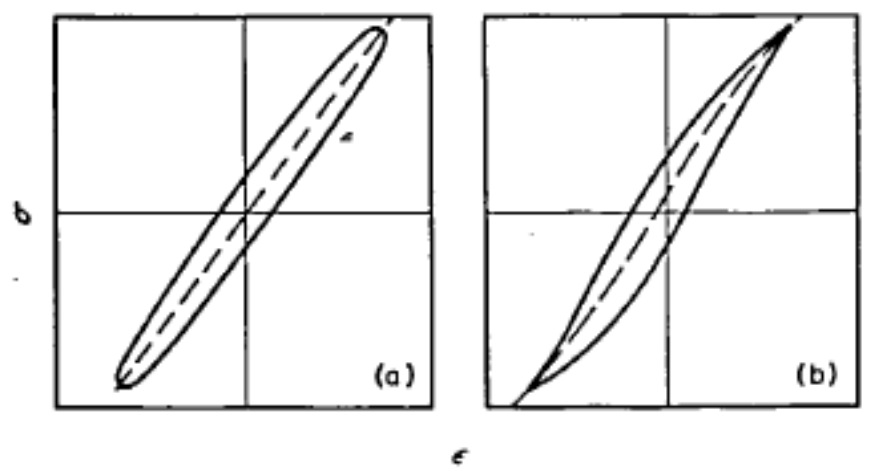
loop.

Figure 3. Hysteresis-loop shapes. (a) Elliptical hysteresis loop, (b) pointed-end, straight-sided hysteresis

Figure 32 - Comparison of frequency-independent hysteresis loops (a) versus typical experimental results (b) taken from Figure 3 of (Bert, 1973)

In Equation 32, the strain rate $\nabla^{s} \mathbf{\mathbf { u }}(\tau)$ is under the integral sign [49], [52], and $\mathbf{G}_{R}(t)$ is the (tensor) relaxation modulus. Using this form, numerous models can be reformulated, including the Kelvin-Voigt model, the Maxwell model, and generalizations such as the Kelvin and Maxwell chains [53], [54], [50], [55] and the Biot model originally investigated by Caughey [49], [56]. Such models can only approach frequency-independent behavior over a limited range. In fact, as shown, for example, by Muscolino [46], it is impossible to generate a linear model which has constant static and dissipation moduli in the frequency domain, and which does not violate causality. Hence the following can be concluded:

1. Standard linear viscous damping (e.g. Rayleigh Damping), although simple to implement, is inadequate because of the strong dependence on frequency, which is not in agreement with experimental observations.

2. Frequency-independent "hysteretic" damping as implemented in SASSI in the frequency domain, although perhaps a better fit to experimental data, is mathematically and physically problematic as it leads to non-physical results when transformed back to the time domain.

3. Various more complicated (yet still linear) extensions using convolution integrals, fractional derivatives, or matrix exponentials, can approach frequency-independence, at the cost of great complication - and with the typical result that frequency-independent dissipation is only achievable over a narrow frequency range.

Of course, none of these models can account for variations in elastic compliance and damping with strain rate, magnitude, and confining pressure, which have been measured experimentally, as for example by EPRI [4].

It is perhaps not surprising that linear models cannot adequately capture the response of foundation soils, as the underlying mechanisms are frictional and hence inherently non-linear; a frictional system does not conform to the principle of superposition. To justify this statement, consider that in classical Coulomb friction, the magnitude of the frictional response is proportional to the displacement, but the sign of the response is opposite to the sign of the velocity. Hence, the superposition of a low-amplitude, high-frequency signal (large velocity and acceleration in comparison to the displacement) will produce a small change in displacement (and hence a small change in the magnitude of the force) but may reverse 
the sign of the velocity, thereby changing the sign of the response. Thus, the principal of superposition will be violated.

An early investigation of frictional models was made Moliner [57] using linear response theory. A simple non-linear frictional model using a regularized form of the Coulomb law was proposed by Dahl [58]. This work was subsequently shown to be one form of a Duhem model, of which there are additional variants, see [59], with rate- (and hence frequency-) independent hysteresis. The Dahl model is seen to provide hysteretic loops with behavior close to the experimentally observed results of Panel (b) in Figure 32, see Figure 33. This provides further confirmation that a non-linear material model based upon the concepts of friction and plasticity, is the right approach towards capturing the essential characteristics of soil response.

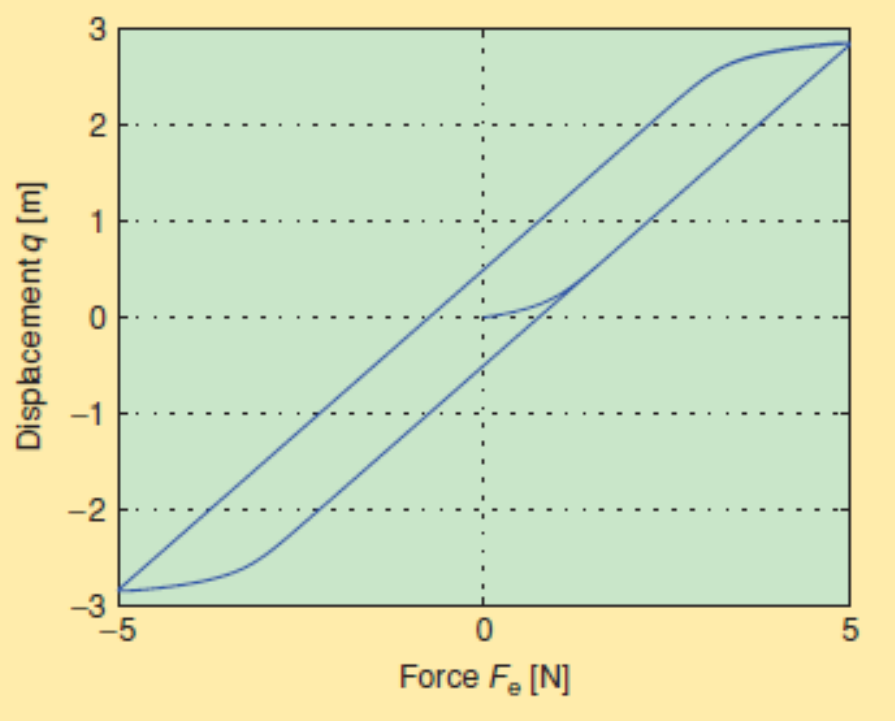

FIGURE 13 Hysteresis map from the external force $F_{\mathrm{e}}(t)$ to the displacement $q(t)$ of the mass for the force-actuated mass-spring system (28) in Figure 12, with the Dahl model. The numerical values are $F_{\mathrm{C}}=0.75 \mathrm{~N}, \gamma=1, \sigma=7.5 \mathrm{~N} / \mathrm{m}, k_{\mathrm{s}}=1.5 \mathrm{~N} / \mathrm{m}, m=1 \mathrm{~kg}$, and $F_{\mathrm{e}}(t)=5 \sin (0.001 t) \mathrm{N}$. The vertical width of the hysteresis map is $1 \mathrm{~m}$.

Figure 33 - Dahl model hysteresis loop - taken from Figure 13 of (Padthe, 2008)

Unfortunately, the Dahl model, although rate-independent, relies on specifying a maximum frictional force $F_{c}$. At a constant frictional force, the dissipation is linear with the maximum strain, whereas the maximum stored energy is quadratic - hence the loss factor is inversely proportional to the maximum strain. That is, for the Dahl model, the dissipation is frequency-independent but (highly) amplitudedependent.

More complicated non-linear mathematical models for hysteresis exist, most prominent among them being Bouc-Wen models, involving the evolution of a secondary "hysteresis" variable via a non-linear evolution law [60] [61], [14]. Emanating primarily from the study of electromagnetics, formal models of hysteresis developed by Jiles-Atherton and Preisach, among others, have also been applied [62], [63]. 
Emanating originally from the study of cyclic plasticity, Masing [64] postulated a self-similarity argument for hysteresis curves. The resulting mathematical description is formally related to Preisach models and Iwan models [65], as shown by [24]. Various authors have developed models for hysteresis based on the general idea of the Masing model but including stiffness and "yield strength" degradation over time, including "peak-oriented", and "pinching". See [66] for a good review, also [67], [68], [69], [23], and [40].

To varying degrees, the Bouc-Wen, Preisach, and Masing models all are based upon a mathematical description of one-dimensional hysteresis loops. Ramberg and Osgood [20] developed one model in this family using a simple set of parameters for modeling stress-strain curves for metals. This model has been successfully used for modeling hysteretic behavior in frame structures, neo-Hookean materials, and soils, [70], [71], [72], [73], [66], [74]. For some recent work, see for example [75] [76] and [24]. In the form implemented in NIKE3D [77] and DIABLO [78], only four parameters are required to characterize the response in one dimension:

\section{Equation 33 - One-dimensional Ramberg-Osgood relation}

$$
\begin{aligned}
& \frac{\gamma}{\gamma_{y}}=\frac{\tau}{\tau_{y}}\left(1+\alpha\left|\frac{\tau}{\tau_{y}}\right|^{r-1}\right) \text { (initial loading) } \\
& \frac{\gamma-\gamma_{0}}{2 \gamma_{y}}=\frac{\tau-\tau_{0}}{2 \tau_{y}}\left(1+\alpha\left|\frac{\tau-\tau_{0}}{2 \tau_{y}}\right|^{r-1}\right) \text { (subsequent loading and reloading) }
\end{aligned}
$$

Where,

\section{Equation 34 - Ramberg-Osgood parameters}

$\gamma_{y}:$ reference shear strain (parameter)

$\tau_{y}:$ reference shear stress (parameter)

$\alpha$ : constant $>0$ (parameter)

r: constant $>1$ (parameter)

$\gamma_{0}$ : shear strain at last stress reversal (continually updated during simulation)

$\tau_{0}$ : shear stress at last stress reversal (continually updated during simulation)

The model is designed to produce typical hysteresis curves in one-dimension, such as are typical of shear waves in soils, see Figure 34. 


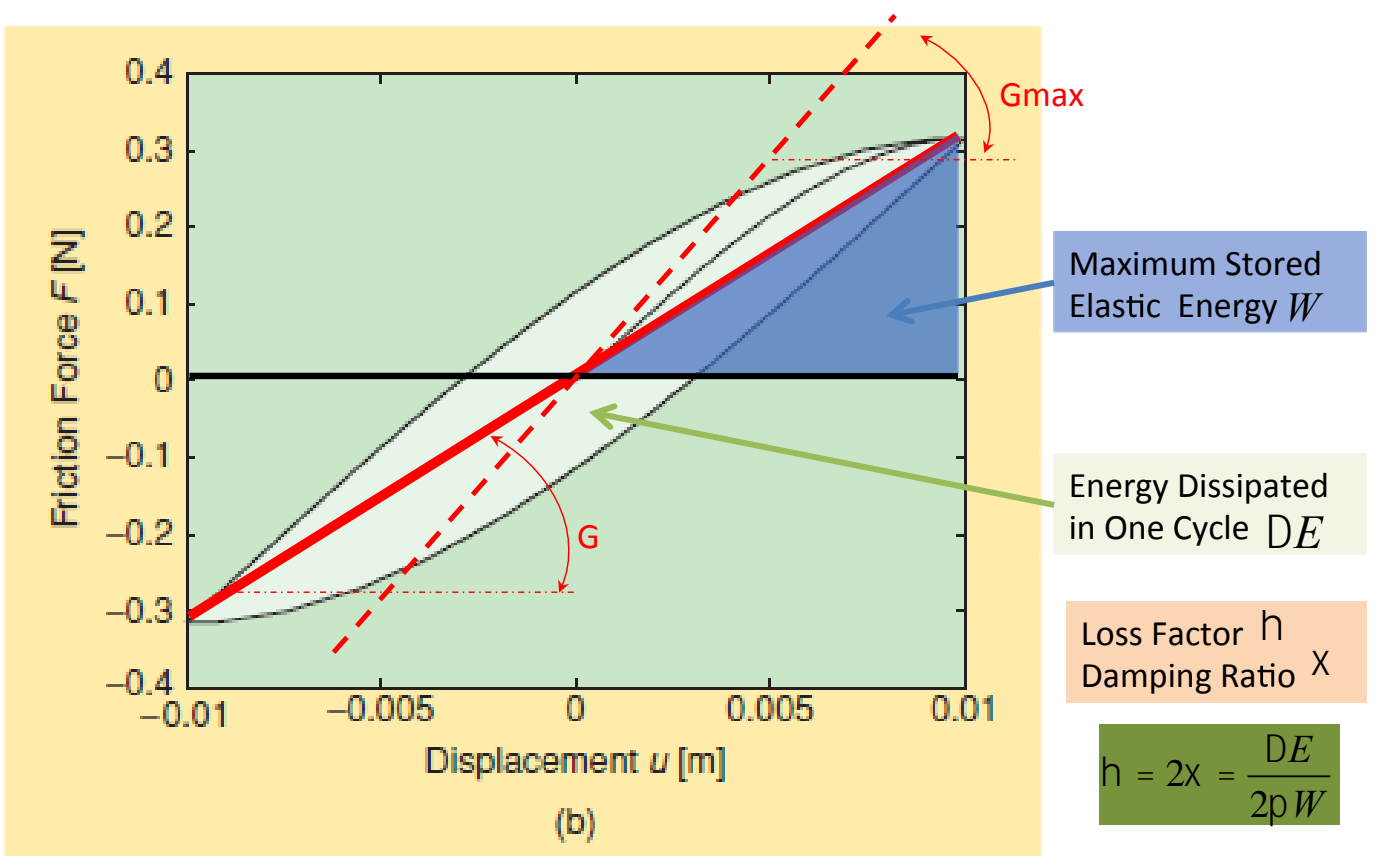

Figure 34 - Typical hysteresis curves with definition of damping

In 1997, Ueng and Chen [21] devised an algorithm that can fit typical shear modulus degradation and damping data [39] as per the EPRI protocol [4] using the Ramberg-Osgood formulation. The algorithm was implemented in the computer code RAMBO. The following characteristics are typical:

- Modulus degradation with increased strain

- Increased damping with increased strain

This is a non-trivial result, because as Phillips remarks in a recent paper [40], "Despite significant efforts to model the hysteretic response and nonlinearity of soils due to medium and large ground motions, the most widely accepted nonlinear site response methods are not able to represent simultaneously the changes of stiffness and energy dissipation (damping) observed in both laboratory tests and during earthquake events".

Like other essentially 1-D models such as the Iwan or Bouc-Wen models, an extension to more than one dimension must be made. In DIABLO and NIKE, the material response is split into an (elastic) volumetric component, the material pressure

Equation 35 - Pressure calculated from the trace of the stress tensor

$$
\mathrm{p}=-\frac{1}{3}\left(\sigma_{11}+\sigma_{22}+\sigma_{33}\right)
$$

and 6 deviatoric stress component which make up the (symmetric) deviatoric stress tensor $\mathbf{s}$ 


\section{Equation 36 - Deviatoric stress}

$$
s_{i j}=\sigma_{i j}+p \delta_{i j}
$$

In DIABLO and NIKE3D, each of the 6deviatoric stress component $s_{i j}$ is evolved separately according to Equation 33. This splitting is simplistic - more sophisticated models (as discussed in Section 3.4.4) would formulate the problem in terms of yield surfaces in three-dimensional stress space. In addition, the implementation of the Ramberg-Osgood model, depending as it does on a splitting of the stress into a deviatoric and a volumetric component, does not incorporate any dependence on confining pressure, as in the well-known critical state theory [79], which is characteristic of sophisticated 3D models of soil response. The Ramberg-Osgood model in its present form was chosen in this pilot study primarily for its simplicity and thus the ease with which EPRI-type data can be fitted, in order to demonstrate the viability of nonlinear analysis in comparison to SASSI. The results of fits to the EPRI data for the considered soil column are discussed in a subsequent section.

In summary,

1. Time-domain codes such as DIABLO naturally utilize Kelvin-Vogt style viscous damping. The dissipation characteristics of such models (increasing dissipation with frequency) are not representative of typical test data. More complicated linear models exist but can only capture purely hysteretic behavior over a limited frequency range.

2. SASSI and other frequency-domain codes typically utilize frequency-independent "hysteretic" damping. Although able to represent certain sets of test data better than viscous models, hysteretic damping is problematic because:

a. The shape of the hysteretic response is not consistent with test data.

b. Hysteretic damping does not have a valid representation in the time domain.

c. Because hysteretic damping relies inherently on the principle of superposition (being valid only in the frequency domain), it cannot adequately account for variations in damping with strain.

3. Models with physically realistic hysteretic response valid in the time domain are naturally nonlinear - this is because hysteresis is the result of friction and plasticity - an inherently non-linear process.

4. Numerous 1-D "mathematical" descriptions exist for hysteresis, including the Dahl/Duhem friction models, Bouc-Wen, Iwan, Masing, Preisach, and Jiles-Atherton models. These models all share an underlying structure, and in many cases the formalism can be shown to be equivalent.

5. A simple 1-D hysteretic model of this family is the "Ramberg-Osgood" model, which can describe realistic, physically-relevant hysteretic loops using a small number of parameters. This model has been extended to 3D for use in the finite element codes DIABLO and NIKE3D.

6. An algorithm for fitting shear modulus degradation and damping curves to the Ramberg-Osgood model has been developed and implemented in the computer code RAMBO.

7. Via this simple formalism, frequency-independent dissipation is achieved and simplistic (but, as it shall be shown, adequate in some cases) shear modulus degradation and damping versus strain behavior is realized, at the additional expense of including nonlinearity.

8. Time-domain codes such as DIABLO naturally accommodate nonlinear material response. 


\subsubsection{Boundary Conditions for Seismic Analyses in Semi-Infinite media}

\subsubsection{A Review of Prior Methods}

One of the primary benefits of SASSI's frequency-domain approach is access to the semi-analytical solution of Waas [22]. The impedance of the free-field response is obtained outside the domain of interest by using this solution to construct the flexibility matrix $F_{p q}$, and inverting it to provide the soil compliance matrix $X_{p q}$. Because this solution is based upon the principle of superposition in the frequency domain, it is restricted to linear material response and frequency-domain solutions.

In the time-domain, various approaches have been used to both input the free-field motions and accommodate the reflections of the free-field motion due to interaction with the structure. A representative example of recent work is the report from Brookhaven [47]. In this work, the explicit finite element code LS-DYNA was compared to a sample SASSI calculation. A representative finite element model from the report is reproduced as Figure 35 and Figure 36. The model consists of a large soil region modeled in half-symmetry surrounding a simple cylindrical reactor vessel.

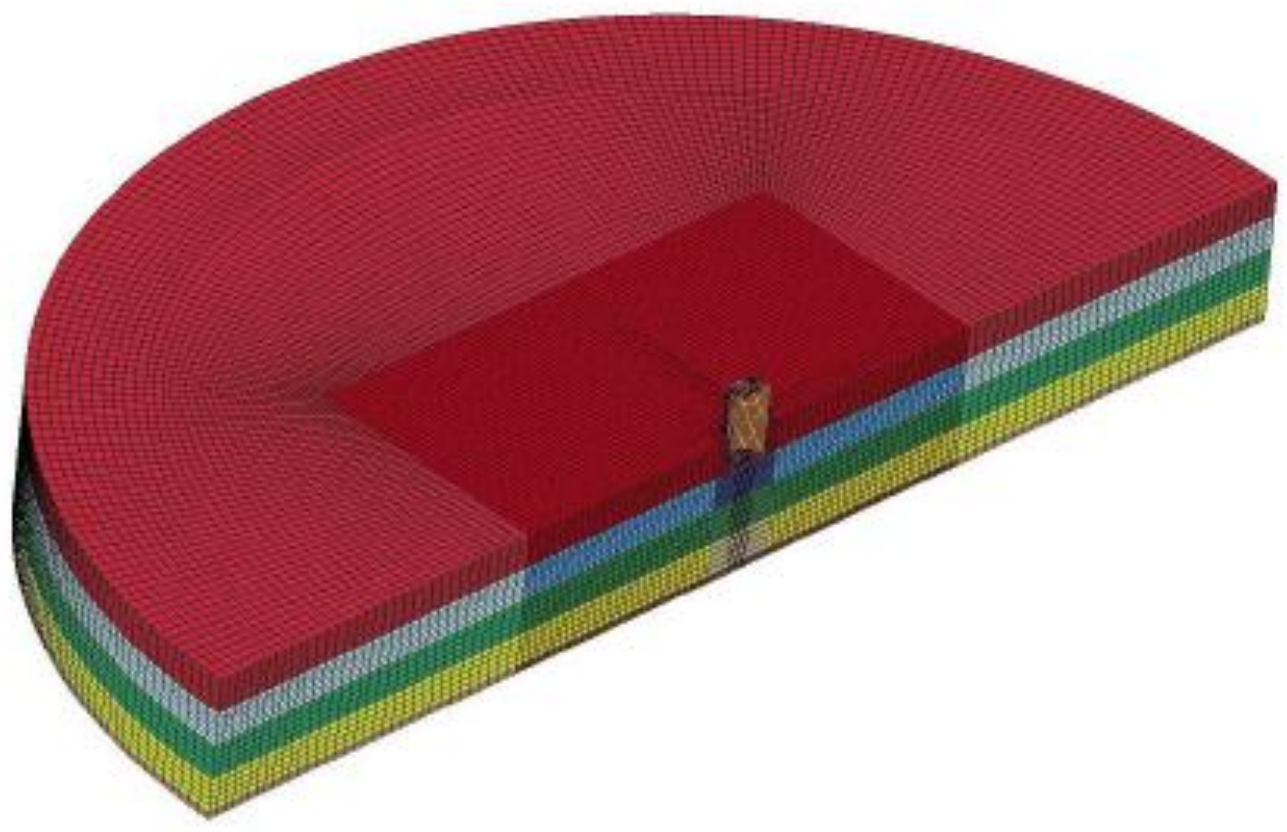

Figure 4.1.2-3 LS-DYNA Model for the $23 \mathrm{~m}$ Embedment.

Figure 35 - Finite element mesh for LS-DYNA analysis - taken from Figure 4.1.2-3 of (Xu, et. al., 2006)

In the Brookhaven study, the bottom four meters of the foundation material was modeled as rock. At the base of the rock and the soil, the acceleration profile of the free-field motion was imposed as a Dirichlet boundary condition ${ }^{11}$.

11 Private communication, Carl Costantino. 
The free-field motions were calculated from given "rock outcrop" motions. These are motions generated either synthetically from a database of suitable earthquakes, or from a wave propagation code following a calculated earthquake from the source through bedrock, but not taking into account local features such as the soil. The rock outcrop motions were used to generate "in-column" motions at the interface, using a 1-D wave propagation code such as SHAKE [5], or CARES [19]. The differences between "rock outcrop" and "in-column" motions are illustrated by Figure 37, taken from Schnabel [32]. In the rock outcrop, illustration (c) in the figure, the reflected wave and incident wave, $E_{N}$, have identical magnitudes. In the full system halfspace, illustration (a) in the figure, the reflected wave $F_{N}$ has a different (lower) magnitude than the incident wave, because of the impedance of the soil. Thus the "incolumn" motion is reduced from the "rock outcrop" motion by some fraction. Figure 38 illustrates the motion reduction [32]. Around a frequency of $1 \mathrm{~Hz}$, the rock base motion as a function of the rock outcrop motion, transfer function $A^{\prime}{ }_{N}$ (dotted line in the figure), has a value of 0.65 . This explains why the base rock to soil surface transfer function, $A_{1, N}$ has a larger magnitude in the figure than the rock outcrop to soil surface transfer function, $A^{\prime}{ }_{1, N}-$ the soil surface response is the same for both, but the rock base motion is less than the (theoretical) rock outcrop motion.

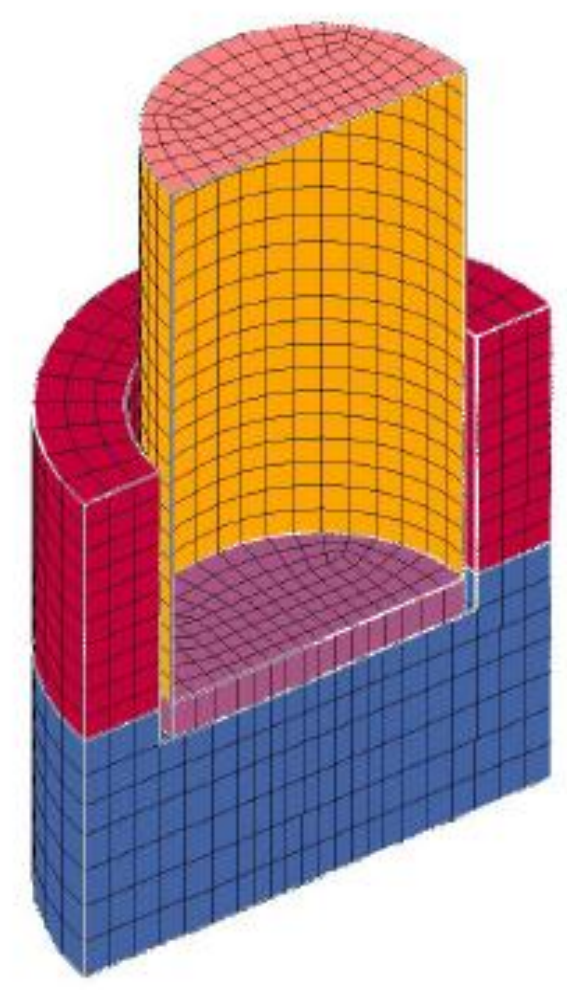

Figure 4.1.2-4 Zoom-in View of the Structure and Surrounding Soil in LS-DYNA Model for the $23 \mathrm{~m}$ Embedment.

Figure 36 - Zoomed in finite element mesh for LS-DYNA analysis - taken from Figure 4.1.2-4 of (Xu, et. al., 2006)

The "in-column" motions then represent the ideal free-field time history along this layer. The typical method (outside of seismic analysis) for imposing a time history of motions would be to impose displacement boundary conditions. In the absence of the structure, imposing the free-field displacements (e.g., standard Dirichlet boundary conditions) at the soil/rock interface, assuming 
appropriate absorbing boundary conditions at the bottom of the mesh, should produce the correct freefield motions everywhere in both the soil and the rock (assuming adequate mesh refinement). However, when the structure is present, the free-field motions will be scattered by the (quite different) impedance of the structure. These scattered motions will propagate to the location where the Dirichlet boundary conditions are imposed. Since the Dirichlet boundary conditions do not allow waves to pass through, the scattered waves will be reflected at the Dirichlet boundary. Hence, Dirichilet boundary conditions are only valid for imposition of motions at a soil/rock interface where the soil is very much softer than the rock interface. ${ }^{12}$

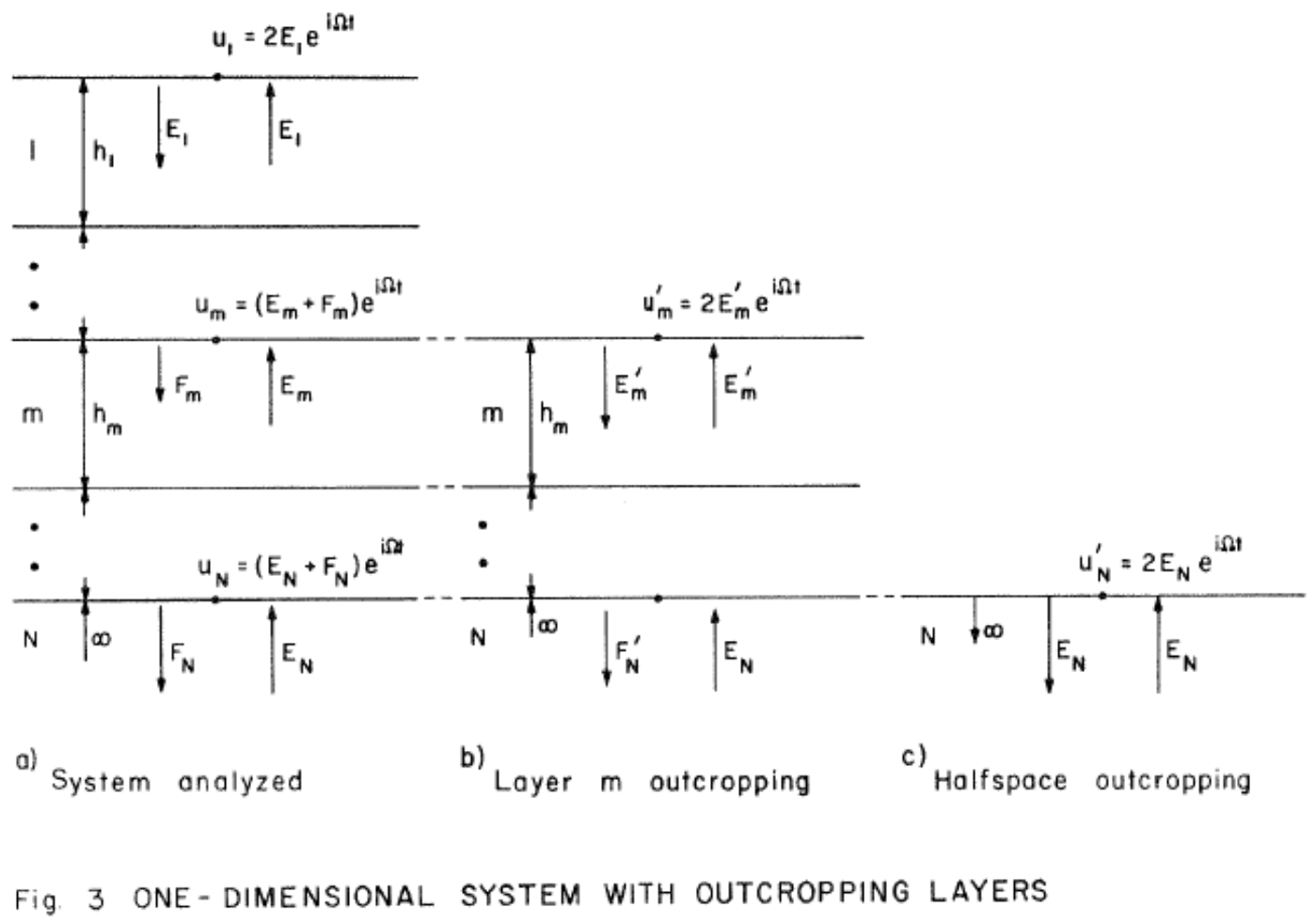

Figure 37 - Rock Outcrop Motions versus Layered Half space - taken from Figure 3 of (Schnabel, 1972)

The standard alternative is "Neumann" boundary conditions (forces) at some point along the mesh. Given the correct forces, the result of reflections from the structure (the scattered wave) will pass through a surface where forces are applied. The derivation of the correct forces is non-trivial, however. Historically the most common method for applying the correct forces was in concert with an absorbing boundary condition. The first notable absorbing boundary condition developed for the finite element method, and still in wide use, is the "Lysmer and Kuhlemeyer" (sometimes known as the "standard

\footnotetext{
${ }^{12}$ Even in the equivalent-linear analysis, the ratio of rock to soil stiffness at which a Dirichlet boundary condition would be appropriate varies with the frequency of the excitation and the magnitude of the earthquake.
} 
viscous" or "LK") boundary condition [80]. In this boundary condition, traction is applied at the boundary according to:

\section{Equation 37 - Lysmer-Kuhlemeyr absorbing boundary condition}

$$
\mathbf{t}=\dot{\mathbf{u}} \rho\left(V_{P}[\mathbf{n} \otimes \mathbf{n}]+V_{S}[\mathbf{I}-\mathbf{n} \otimes \mathbf{n}]\right)
$$

Given an imposed displacement time history $\dot{\mathbf{u}}^{\prime}(t)$, the boundary condition can be "run backwards" to produce a forcing function $\mathbf{t}^{\prime}$ that can be used to drive the solution, via

Equation 38 - Driving forces calculated based upon by "driving backwards" the Lysmer boundary condition

$$
\mathbf{t}^{\prime}(t)=\left(\dot{\mathbf{u}}(t)-\dot{\mathbf{u}}^{\prime}(t)\right) \rho\left(V_{P}[\mathbf{n} \otimes \mathbf{n}]+V_{S}[\mathbf{I}-\mathbf{n} \otimes \mathbf{n}]\right)
$$

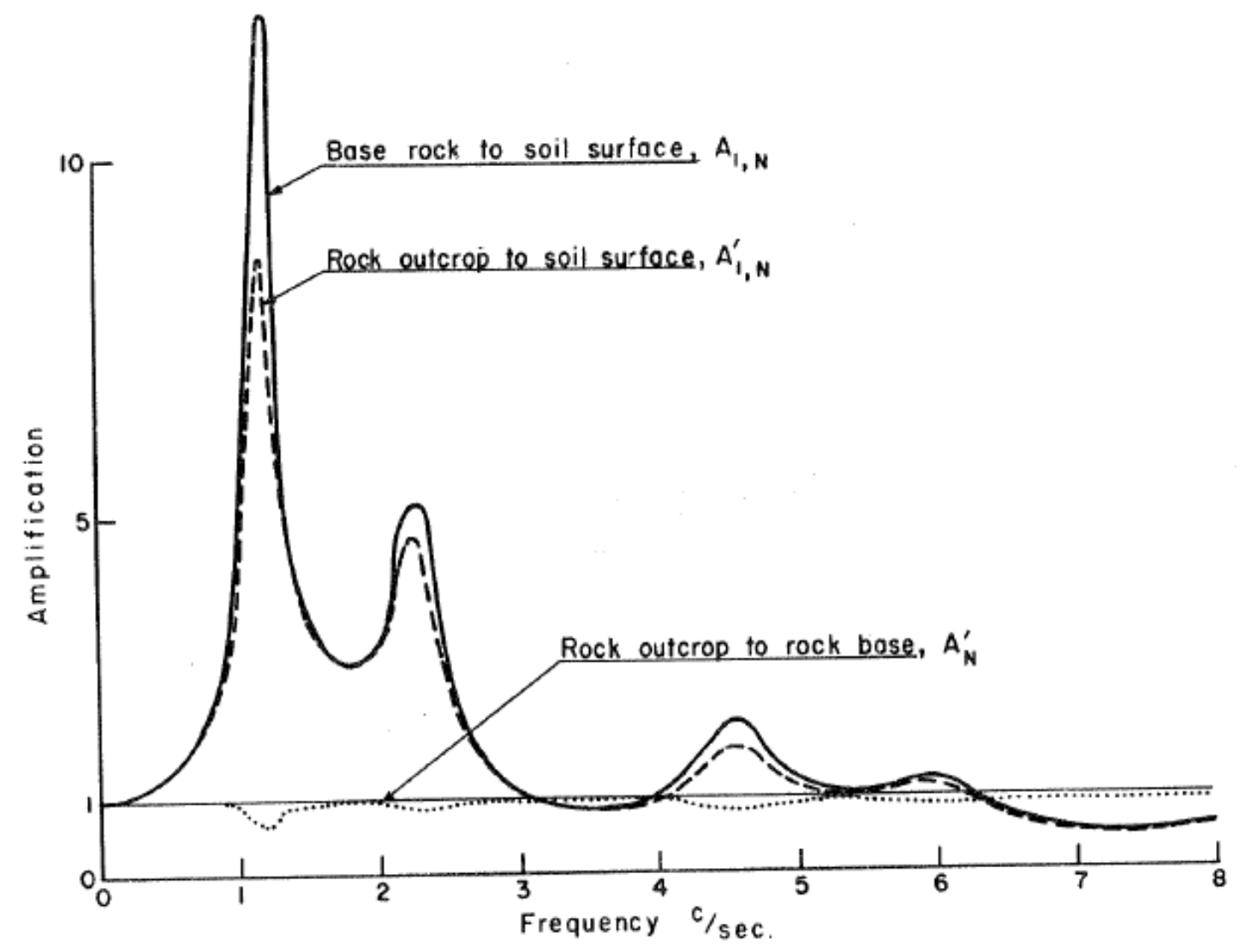

Fig. 4 TRANSFER FUNCTIONS

Figure 38 - Transfer functions for rock outcrop versus rock base - taken from Figure 4 of (Schnabel, 1972) 
Clayton and Enquist [81] developed higher-order versions of the LK boundary condition. A good review of the pioneering work in this field is provided by [82], see also Figure 39. In this figure, the magnitude of the reflected wave (normalized to the magnitude of the incident wave) is compared for various techniques. The domain has a ratio of height to width ${ }^{13} \mathrm{~S} / \mathrm{H}=5$, and boundary conditions include the Dirichlet ("Fixed") boundary, the homogeneous Neumann boundary ("Free"), the Smith boundary (a combination of Dirichlet and Neumann terms), and 3 orders of the Clayton-Enquist boundary condition (the first order approximation, "First Approximation", corresponds to the Lysmer boundary condition of Equation 37).

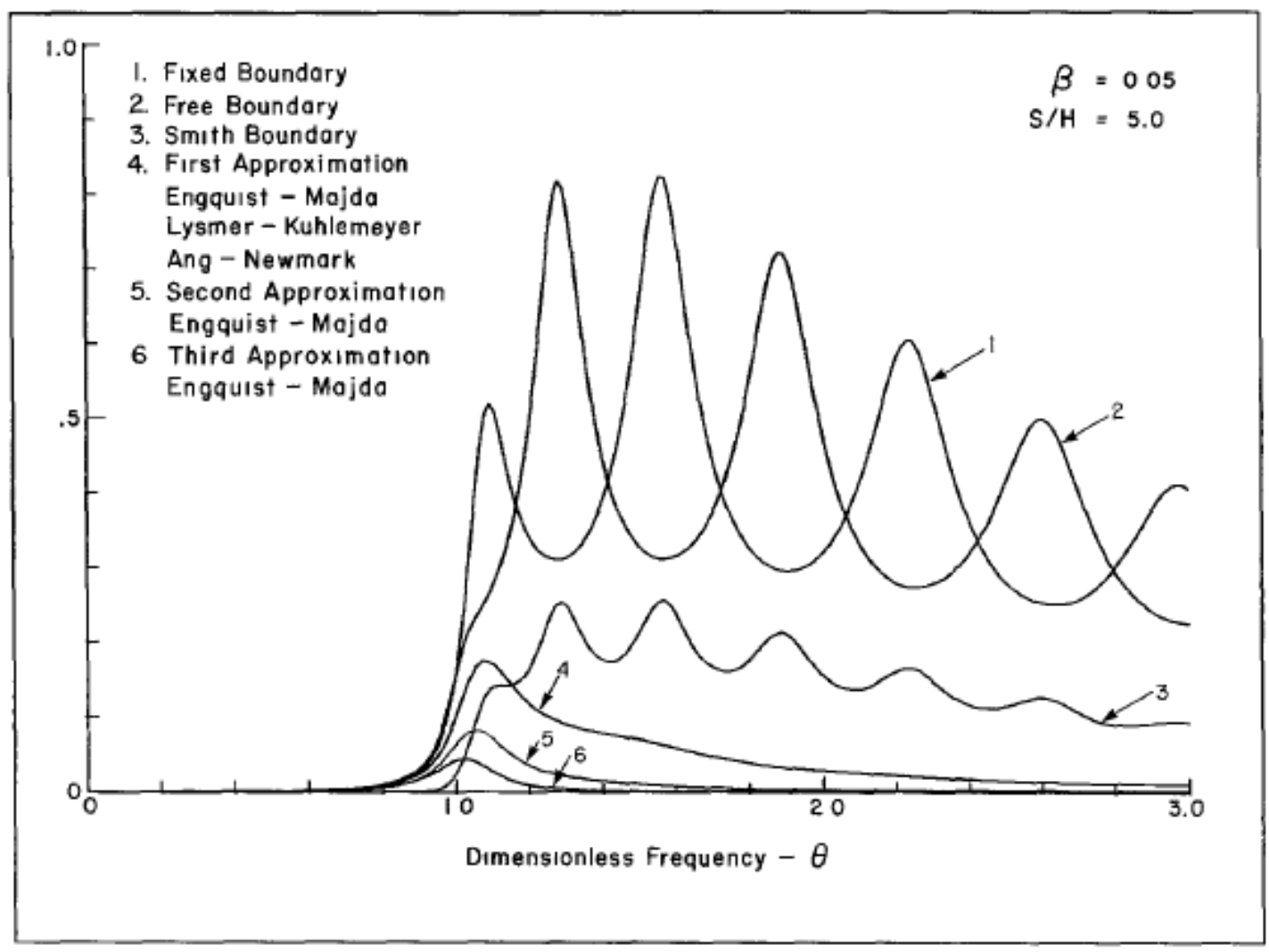

Fig. 6. Normalized amplitude of reflected fundamental mode, $S / H=5$.

Figure 39 - Performance of various simple absorbing boundary conditions - taken from Figure 6 of (Kausel, 1981)

From the figure, it is clear that whereas the LK boundary condition does a reasonable (but not perfect) job of absorbing the reflected wave, the "Fixed", "Free", and "Smith" boundary conditions are inadequate. Typically the boundary conditions are worst at or around the dimensionless frequency ${ }^{14}$, $\Theta$, of 1.0. Clayton-Enquist's higher-order boundary conditions are even more absorbent than the Lysmer boundary condition, but are difficult to implement in a finite element context (as opposed to finite-difference). More comprehensive reviews of work in this field are provided by Kausel [83] and Givoli ( [84], [85]. The latest work in higher-order absorbing boundary conditions valid in the time-

${ }^{13} \mathrm{~S}$ refers to the half-width of the domain, $\mathrm{H}$ is the height of the domain, see Figure 3 of [82].

${ }^{14}$ Frequency normalized by the natural frequency of the first mode of the soil column 
domain is reviewed and further developed in the recent papers by Rabinovich, Bielak, Givoli and coworkers [86], [87].

Kellezi [88] is one of a number of authors who tackles one of the major downfalls of the Lysmer and similar boundary conditions for nonlinear analysis - as a purely "viscous" boundary, it has no static stiffness. Hence the Lysmer boundary condition by itself cannot be used in the context of static (gravitydriven) loading situations. For a linear analysis this is not particularly a problem, as the results of a static analysis can be superimposed upon the results of the dynamic analysis. For nonlinear problems, static boundary stiffness is required. One possibility involves peforming a dynamic analysis using the initial conditions initialized from a static analysis performed using Dirichlet boundary conditions at the boundary, but with the Dirichlet boundary conditions replaced in the dynamic analysis with forces calculated from the static resultants.

An alternative to a restart-based approach are various "foundation boundary condition" (boundary stiffness) formulations as implemented in, for example, [89]). In this paper, alternative treatments are reviewed, such as Infinite Element methods [90], [91]. Application of Infinite Elements to timedependent problems is typically performed in the frequency domain, producing solutions in terms of Hankel functions similar in many respects to the thin layer method, [92], [93], [94], and also requiring inverse transforms to produce a time-domain solution. Su and coworkers [95] have recently proposed a formulation where the static response of the infinite elements and the dynamic response of absorbing boundary conditions of the LK type are combined, in order to avoid the inverse transforms in the time domain required for the standard infinite element approach.

An alternative to absorbing boundary conditions, boundary elements, and infinite elements that has recently been developed is based on the idea of "Perfectly Matched Layers", or PMLs, originally developed for the electromagnetic wave equation by Berenger [96]. The PML represents a finite element uniquely tuned to damp out incident waves. It has been extended to elastodynamics by a number of authors, among them Basu [97], [98], and Kucukcoban [99]. In the latter two works, the PML has been developed originally in the frequency-domain, and the time-domain equations have been derived using an inverse Fourier transform. An interesting application of Caughey's method has been utilized by Semblatt to derive a novel absorbing layer directly in the time domain [100]. Festa pioneered a direct approach in velocity-stress space [101] [102]. PMLs are still an open area of research, and available algorithms typically do not perform well in the case of grazing incidence and anisotropic media [103]. In addition, the typical formulation, relying on an inverse transform, restricts them to linear material behavior as they rely on the principle of superposition.

The emphasis placed upon the limitation of most of the absorbing boundary conditions to linear material response is based upon the observation made in Section 3.2.3 that the damping characteristics of soils, even at small strains, is only effectively modeled by non-linear hysteretic soils in the timedomain. Since the "impedance" of a soil is a function of the damping characteristics, effective "impedance matching" required for an absorbing boundary condition requires some ability to match the inherently non-linear characteristics of the soil. This will be addressed in more detail in a subsequent section. 
In summary,

1. The related problems of defining the seismic input and creating appropriate absorbing boundary conditions for seismic analysis in the time domain has been an open area of research since the late 1960s.

2. The original "LK" boundary conditions of Lysmer and Kuhlemeyer are simple to implement and reasonably effective. They can also be "driven backwards" to produce effective seismic input.

3. Numerous higher-order boundary conditions have been derived subsequently. These include higher-order absorbing boundary conditions, infinite elements, and perfectly matched layers. These all involve significant complication, and most are inherently restricted to linear material behavior because they rely on inverse Fourier transforms.

4. Because the damping characteristics of a soil are inherently hysteretic (and thus nonlinear), the linear basis of most absorbing boundary conditions greatly impedes their ability to properly "impedance match" such soils and hence adequately absorb waves.

5. Given the imperfect nature of these absorbing boundaries, especially for nonlinear soils, the problem of seismic input remains difficult, since the typical solution of "driving the absorbing boundary condition backwards" is also therefore inadequate.

\subsubsection{The Original Bielak Method}

The preceding section has demonstrated the difficulty in developing ideal absorbing boundary conditions, especially for problems involving nonlinear materials. As a side effect of this difficulty, the imposition of appropriate seismic loads for problems with nonlinear materials is also non-trivial. One approach, developed by Bielak and coworkers [16], [104], [17], [105] imposes the motion on a subset of the analysis (the "soil island"), yet allows for scattered waves to propagate outwards through a larger (yet still small compared to geologic scales) domain, only to be finally absorbed at boundaries located at some distance.

To understand the wisdom of this approach, consider Figure 40. The analysis region is split into two domains, an internal domain labeled as $\Omega_{I}$ in the picture, which includes the structure to be analyzed, and a surrounding region labeled as $\Omega_{I I}$ in the picture, separated by the surface $\Gamma_{b}$. The boundary of the external region (minus the free surface of the soil) is then denoted by $\Gamma_{e}$, where an absorbing boundary condition is imposed. 


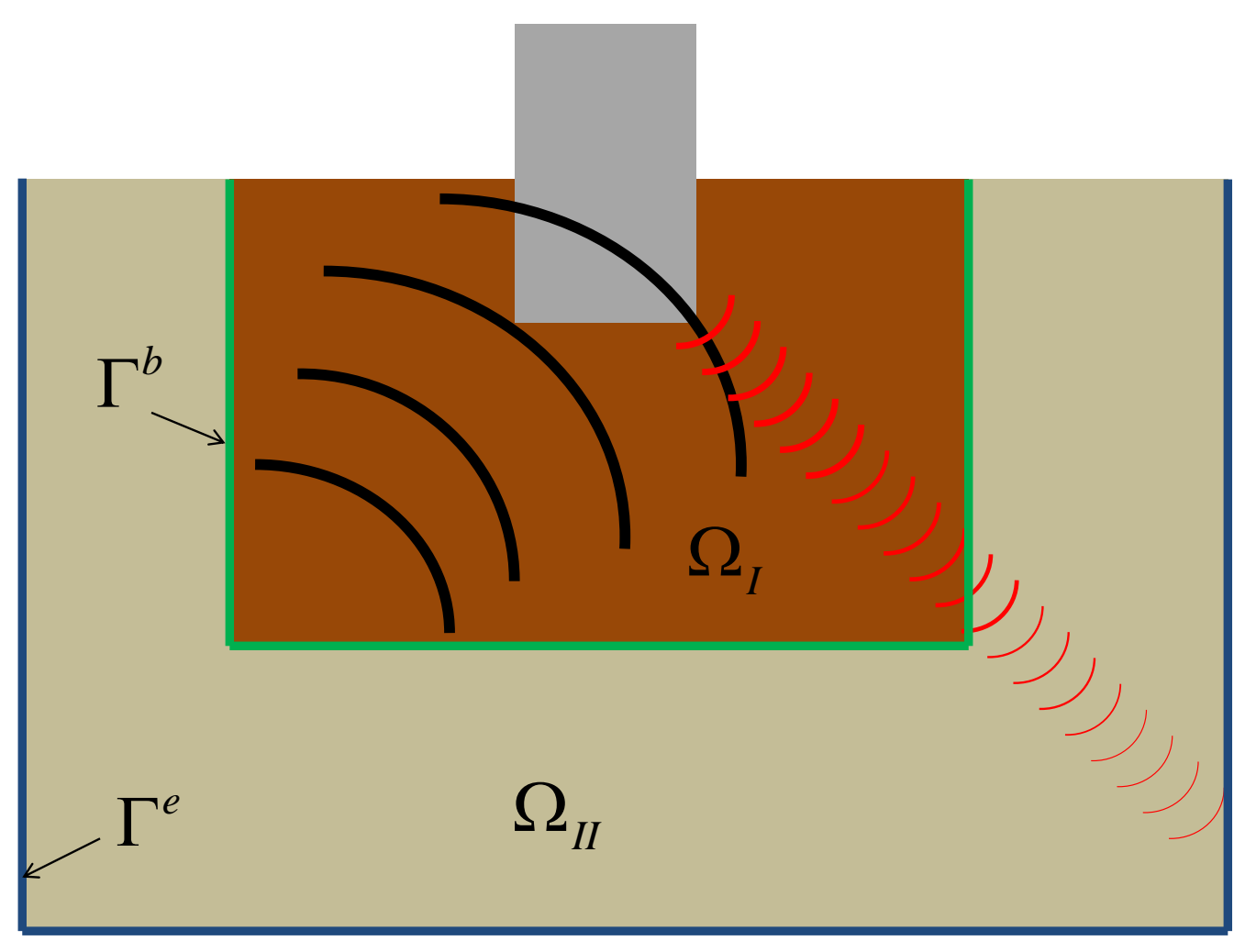

Figure 40 - Bielak method, conceptual approach

As conceived by Bielak and coworkers, the seismic wave is input along $\Gamma^{b}$ such that it only acts within the internal region $\Omega_{I}$. Assuming the seismic input corresponds to the exact solution for a seismic wave propagating in $\Omega_{I}$, in the absence of the structure, the displacements within the external region $\Omega_{I I}$ would be zero. Given the presence of the structure, the seismic wave is scattered, and these scattered waves propagate back through $\Omega_{I}$ to the internal boundary $\Gamma^{b}$. Assuming the material properties within $\Omega_{I}$ and $\Omega_{I I}$ along $\Gamma^{b}$ are impedance-matched, the scattered waves passes through $\Gamma^{b}$ unimpeded, and propagates through $\Omega_{I I}$ to the outer boundary $\Gamma^{e}$, where it is absorbed.

This algorithm has the following key features:

1. Knowledge of the free-field motion is needed only in a small subset of the problem, in this case the surface $\Gamma^{b}$.

2. In the absence of the structure, the exact solution for the seismic wave is reproduced within $\Omega_{I}$, and zero displacement within $\Omega_{I I}$ (assuming the seismic input corresponds to the exact solution for a seismic wave within $\Omega_{I}$ ).

3. The need for a high-performance absorbing boundary is greatly reduced, since

a. The seismic input does not require an absorbing boundary condition to be "driven backwards" as in Equation 38, since the seismic input is applied along $\Gamma^{b}$ using another technique. 
b. The only waves that propagate within $\Omega_{I I}$ are the scattered waves, which are of significantly reduced magnitude in comparison to the seismic input.

c. As both $\Omega_{I}$ and $\Omega_{I I}$ include dissipation, the magnitude of the scattered waves by the time they reach $\Gamma^{e}$ is small, even when compared with the magnitude at the structure (the source of the scattered waves).

As originally implemented, the Bielak Method involved an additional region $\Omega_{I I I}$ adjacent to $\Gamma^{b}$, and assumed 1 element thick, see Figure 42.

The Bielak method begins by assuming the existence of an exact solution in terms of the displacement $\overline{\mathbf{u}}(\mathbf{x}, t)$, as a function of position and time, to the free-field wave propagation problem, known at a minimum within the region $\Omega_{I I I}$. Via standard kinematics, the symmetric small strain tensor $\nabla^{s} \overline{\mathbf{u}}$ and its time derivative $\nabla^{s} \dot{\overline{\mathbf{u}}}$ are also known, also as a function of time and position. Through the constitutitve relation for the material, this gives rise to knowledge of the Cauchy Stress $\boldsymbol{\sigma}$, also as a function of time and position - that is

$$
\begin{aligned}
& \mathbf{u}=\overline{\mathbf{u}}(\mathbf{x}, t) \Rightarrow \dot{\mathbf{u}}=\dot{\overline{\mathbf{u}}}(\mathbf{x}, t) \Rightarrow \ddot{\mathbf{u}}=\ddot{\overline{\mathbf{u}}}(\mathbf{x}, t) \\
& \boldsymbol{\sigma}=\boldsymbol{\sigma}\left(\nabla^{s} \overline{\mathbf{u}}, \nabla^{s} \dot{\overline{\mathbf{u}}}\right) \Rightarrow \boldsymbol{\sigma}=\overline{\boldsymbol{\sigma}}(\mathbf{x}, t)
\end{aligned}
$$

\section{Figure 41 - Bielak method assumed exact solution}

Because the region $\Omega_{I I I}$ is assumed one element thick, the nodes associated with the elements in $\Omega_{I I I}$ fall into two categories, those associated with the "seismic input" boundary $\Gamma^{b}$, and those associated with the boundary with $\Omega_{I I}, \Gamma^{\circ}$, as in Figure 42. Construct a smooth non-negative scalar function $\beta(\mathbf{x})$ that has the value 1 along $\Gamma^{b}$ and the value 0 along $\Gamma^{o}$, that is

\section{Equation 39 - Scaling function}

$$
B(\mathbf{x}):=\left\{\begin{array}{l}
1 \forall \mathbf{x} \in \Gamma^{b} \\
0 \forall \mathbf{x} \in \Gamma^{0}
\end{array}\right.
$$

The scaling function is used to produce a scaled solution within $\Omega_{I I I}$

\section{Equation 40 - Scaled Solution}

$$
\begin{aligned}
& \tilde{\mathbf{u}}(\mathbf{x}, t)=\beta(\mathbf{x}) \bar{u}(\mathbf{x}, t) \\
& \tilde{\boldsymbol{\sigma}}=\boldsymbol{\sigma}\left(\nabla^{s} \tilde{\mathbf{u}}, \nabla^{s} \dot{\tilde{\mathbf{u}}}\right)=\tilde{\boldsymbol{\sigma}}(\mathbf{x}, t)
\end{aligned}
$$




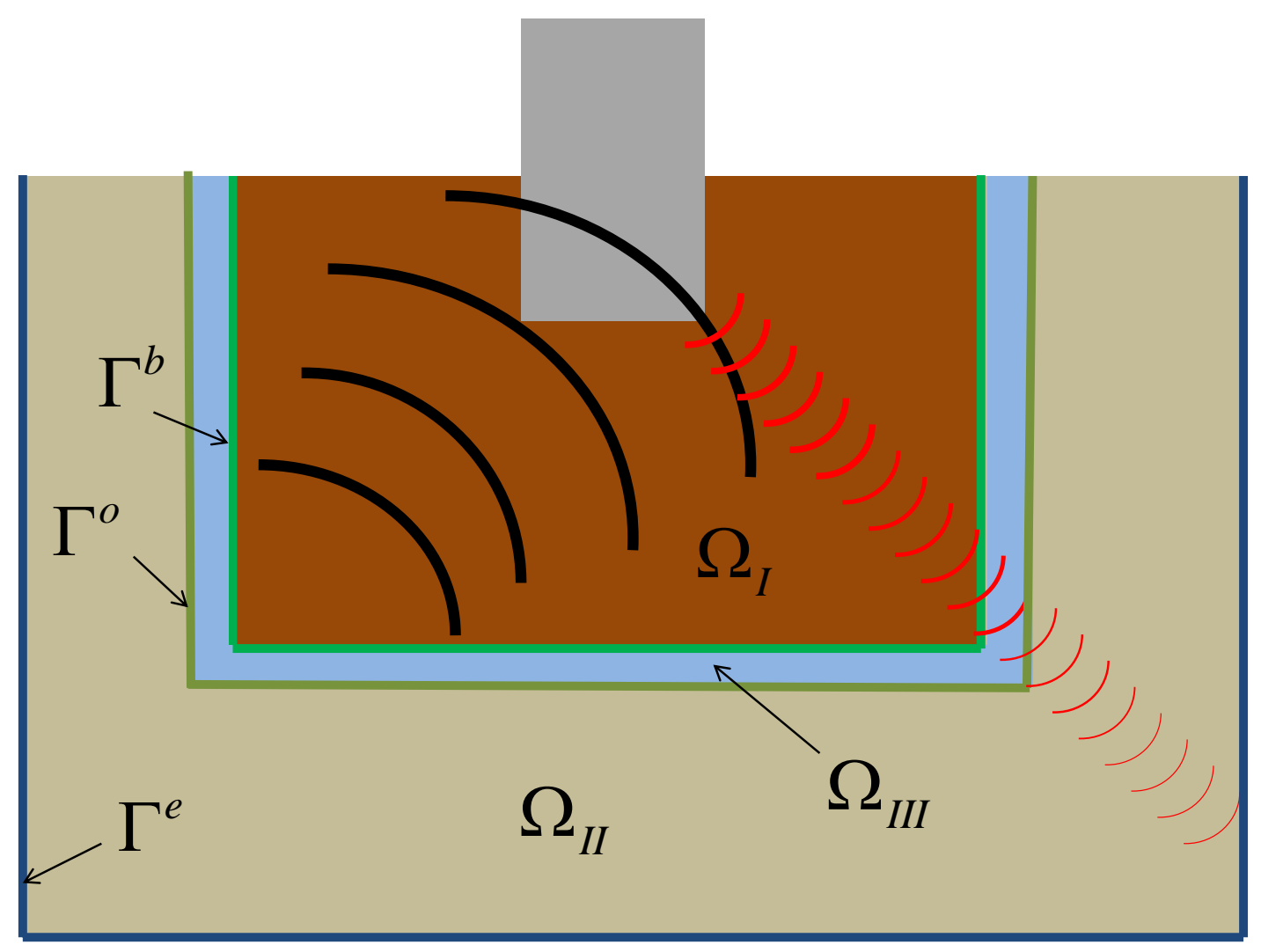

Figure 42 - Bielak method showing the intermediate region

For nodes that are NOT members of $\Gamma^{b}$, the forces due to the scaled solution are subtracted from the current solution. For nodes that ARE members of $\Gamma^{b}$, the forces due to the scaled solution are subtracted, and then the forces due to the exact solution are added. In other words, Equation 5 is modified to ${ }^{15}$

$$
\begin{gathered}
\text { Equation } 41 \text { - Nodal Forces for Bielak Method } \\
{[F]=\left\{\begin{array}{c}
-\left.\int_{\Omega_{I I}}\left((\boldsymbol{\sigma}-\tilde{\boldsymbol{\sigma}}) \bullet \nabla^{S}\left[\mathbf{e}_{i} N^{I}\right]+\rho(\ddot{\mathbf{u}}-\ddot{\tilde{\mathbf{u}}}) \bullet \mathbf{e}_{i} N^{I}\right) d \Omega\right|_{\text {node a member of } \Omega_{\mathrm{III}} \text { but is not a member of } \Gamma^{b}} \\
-\int_{\Omega_{I I}}\left((\boldsymbol{\sigma}-\tilde{\boldsymbol{\sigma}}+\overline{\boldsymbol{\sigma}}) \bullet \nabla^{S}\left[\mathbf{e}_{i} N^{I}\right]+\rho(\ddot{\mathbf{u}}-\ddot{\tilde{\mathbf{u}}}+\ddot{\overline{\mathbf{u}}}) \bullet \mathbf{e}_{i} N^{I}\right) d \Omega \mid \\
\text { node is a member of } \Gamma^{b}
\end{array}\right.}
\end{gathered}
$$

In the absence of scattered waves from the structure, this results in the following solution

\footnotetext{
15 Note that for an intermediate layer one element thick, the set of nodes which are members of the intermediate layer but not a member of the inner boundary is equivalent to the set of nodes on the boundary with the outer region, that is $\Omega_{I I I} / \Gamma^{b}=\Gamma^{o}$
} 


\section{Equation 42 - Bielak solution in the absence of scattered waves}

$$
\mathbf{u}(x, t)=\left\{\begin{array}{c}
\overline{\mathbf{u}}(\mathbf{x}, t) \forall \mathbf{x} \in \Omega_{I} \\
\tilde{\mathbf{u}}(\mathbf{x}, t) \forall \mathbf{x} \in \Omega_{I I I} \\
\mathbf{0} \forall \mathbf{x} \in \Omega_{I I}
\end{array} \Rightarrow \boldsymbol{\sigma}(x, t)=\left\{\begin{array}{c}
\overline{\boldsymbol{\sigma}}(\mathbf{x}, t) \forall \mathbf{x} \in \Omega_{I} \\
\tilde{\boldsymbol{\sigma}}(\mathbf{x}, t) \forall \mathbf{x} \in \Omega_{I I I} \\
\mathbf{0} \forall \mathbf{x} \in \Omega_{I I}
\end{array}\right.\right.
$$

If scattered waves are generated by the structure, they will perturb the solution everywhere, thus inducing non-zero solutions within $\Omega_{I I}$. However, they will be generally significantly smaller than the incident waves. In addition, because of material dissipation and geometric attenuation, by the time these waves reach the external boundary $\Gamma^{e}$, their magnitude will be reduced further. Hence the absorbing boundary condition need not be exceptionally effective at attenuating reflections at the external boundary.

In summary,

1. The method developed by Bielak and coworkers successfully generates seismic input at an inner boundary.

2. The method requires the identification of a special 1-element intermediate layer, just outside the inner boundary.

3. Assuming linear material behavior adjacent to the inner boundary, only scattered waves (the result of the presence of the structure) will be present in the outer region.

4. Because of geometric and material attenuation, and since the magnitude of scattered waves is typically less than the seismic input, by the time the scattered waves reach the outer boundary they will be of low magnitude, therefore an exceptionally effective absorbing boundary condition is not required.

\subsubsection{An Extension of the Bielak Method for Nonlinear Materials}

The Bielak method was originally formulated for linear materials [17], and for an intermediate region $\Omega_{I I I}$ one element thick. The equation presented above for the forces applied to the internal boundary nodes, Equation 41, is equivalent to the original equations [17] which were in matrix form. Section 3.2.3 has established that material non-linearities have to be accounted for even at very small strains, since the dissipation characteristics of soils are dominated by friction and plasticity, which cannot be adequately modeled linearly. Since Equation 41 has been written without assuming linearity, it can be used for nonlinear materials without any modifications.

However, this is not the ideal for a number of reasons. Most importantly, for proper wave absorption in a nonlinear context, the impedance should vary smoothly across the intermediate region $\Omega_{I I I}$, see Figure 43. For clarity, it is best to delineate the logic behind this argument:

1. For a large earthquake, the imposed seismic input will produce large strains in the material within $\Omega_{I}$ adjacent to $\Gamma^{b}$. 
2. Because of the strain softening and high damping characteristic of soil materials, the impedance of the material within $\Omega_{I}$ adjacent to $\Gamma^{b}$ will differ, perhaps substantially, from material at the zero strain condition.

3. In order to ensure non-reflection of scattered waves as they leave $\Omega_{I}$, $\operatorname{cross} \Gamma^{b}$, and enter $\Omega_{I I I}$, the impedance of the material within $\Omega_{I I I}$ adjacent to $\Gamma^{b}$ should match this softened and highly damped condition.

4. The material within $\Omega_{I I}$ adjacent to $\Gamma^{o}$ is at nominally a zero strain condition, hence its material response is characterized by stiff response and low damping. In order to ensure non-reflection of scattered waves as they leave $\Omega_{I I I}$, $\operatorname{cross} \Gamma^{0}$, and enter $\Omega_{I I}$, the impedance of the material in $\Omega_{I I I}$ adjacent to $\Gamma^{o}$ should match this unstrained condition.

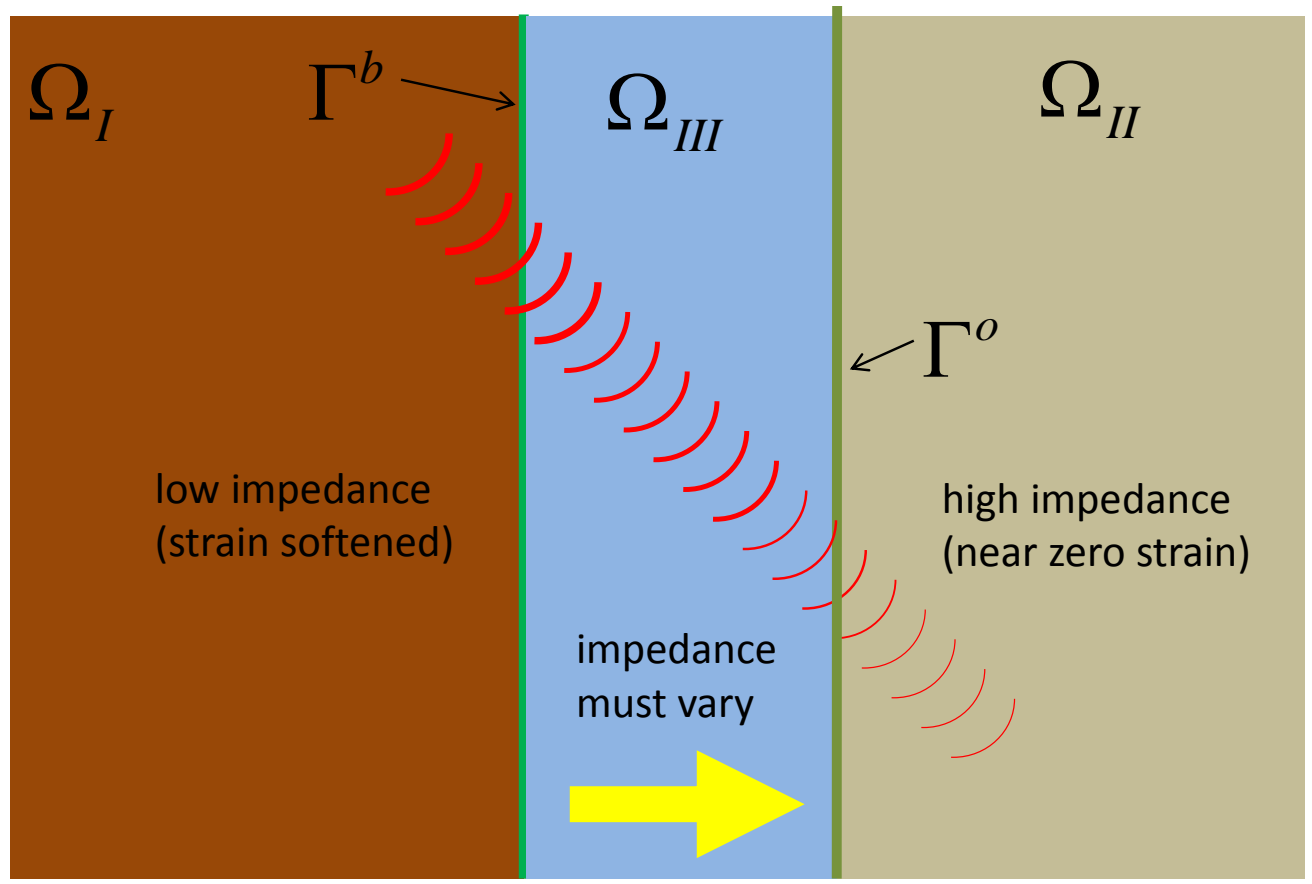

Figure 43 - Impedance must vary within the intermediate layer

For the scattered waves to be non-reflected, the variation of the impedance within $\Omega_{I I I}$ should be gradual and smooth - obviously not possible within the context of a one-element-thick layer. Therefore the first modification for a non-linear context is to modify the Bielak method so that it is valid for a region $\Omega_{I I I}$ more than one element thick. This requires merely defining the function $\beta(\mathbf{x})$ in Equation 39 smoothly in the interior of $\Omega_{I I I}$, for example 
Equation 43 - $\mathrm{B}(\mathrm{x})$ smoothly extended within the interior of $\Omega_{I I I}$

$$
B(\mathbf{x}):=\left\{\begin{array}{c}
1 \forall \mathbf{x} \in \Gamma^{b} \\
0 \forall \mathbf{x} \in \Gamma^{0} \\
\text { smooth,concave,positive } \forall \mathbf{x} \in \operatorname{int}\left(\Omega_{I I I}\right)
\end{array}\right.
$$

However, it turns out this is not quite sufficient. In particular it is found that the stress for the scaled solution as defined by Equation 40 does not vary smoothly in the expected fashion. This result can be explained by examining the strain induced by the scaled solution, which has an extra term $(\overline{\mathbf{u}} \otimes \nabla \beta)^{S}$ generated by the gradient of $\beta(\mathbf{x})$

\section{Equation 44 - Scaled solution has an extra term}

$$
\begin{aligned}
& \tilde{\mathbf{u}}(\mathbf{x}, t)=\beta(\mathbf{x}) \overline{\mathbf{u}}(\mathbf{x}, t) \\
& \Rightarrow \nabla^{s} \tilde{\mathbf{u}}=\beta(\mathbf{x}) \nabla^{s} \overline{\mathbf{u}}+(\overline{\mathbf{u}} \otimes \nabla \beta)^{S}
\end{aligned}
$$

Hence, using scaled solution for the displacements, Equation 40, the stress within the intermediate layer is NOT a scaled version of the stress for the exact solution, even if the material response is linear.

\section{Equation 45 - Intermediate stress is not a scaled version of the exact stress}

$$
\tilde{\boldsymbol{\sigma}}=\boldsymbol{\sigma}\left(\nabla^{s} \tilde{\mathbf{u}}, \nabla^{s} \dot{\tilde{\mathbf{u}}}\right) \neq \beta(\mathbf{x}) \boldsymbol{\sigma}\left(\nabla^{s} \overline{\mathbf{u}}, \nabla^{s} \dot{\overline{\mathbf{u}}}\right)
$$

The perturbation away from a scaled version of the stress is a function of the gradient of $\beta(\mathbf{x})-$ the perturbation is more severe for thinner intermediate regions. For linear materials this difference doesn't matter, because the stiffness and damping characteristics of a material point do not change as a function of stress state. For nonlinear materials, on the other hand, the difference could be large. For materials formulated using advanced pressure-dependent relationships, the difference may even be catastrophic, since the term $(\overline{\mathbf{u}} \otimes \nabla \beta)^{S}$ may induce tension which may cause the material in the intermediate layer to fail.

Fortunately, a solution exists. Within the intermediate layer, employ the following modified strain $\hat{\boldsymbol{\varepsilon}}$ and strain rate $\dot{\hat{\boldsymbol{\varepsilon}}}$ functions

\section{Equation 46 - Modified Strain and strain rate}

$$
\begin{aligned}
& \hat{\mathbf{\varepsilon}}\left(\nabla^{s} \mathbf{u}\right)=\nabla^{s} \mathbf{u}(\mathbf{x}, t)-(\overline{\mathbf{u}} \otimes \nabla \beta)^{s} \\
& \dot{\hat{\boldsymbol{\varepsilon}}}\left(\nabla^{s} \dot{\mathbf{u}}\right)=\nabla^{s} \dot{\mathbf{u}}(\mathbf{x}, t)-(\dot{\overline{\mathbf{u}}} \otimes \nabla \beta)^{s}
\end{aligned}
$$

These modified functions are designed such that when the displacement within the intermediate layer is equal to the scaled solution $\tilde{\mathbf{u}}$, the modified strain function is proportional to the exact solution strain 


\section{Equation 47 - Modified strain is proportional to exact solution strain}

$$
\mathbf{u}=\tilde{\mathbf{u}} \Rightarrow \hat{\boldsymbol{\varepsilon}}=\beta \nabla \overline{\mathbf{u}}
$$

Therefore, in contrast to Equation 45, for a linear material the stress calculated using the modified strain and strain rate is proportional to the exact solution

\section{Equation 48 - Stress calculated using the modified strain is proportional to the exact solution for a linear} material

$$
\boldsymbol{\sigma}\left(\hat{\boldsymbol{\varepsilon}}\left(\nabla^{s} \tilde{\mathbf{u}}\right), \dot{\hat{\boldsymbol{\varepsilon}}}\left(\nabla^{s} \dot{\tilde{\mathbf{u}}}\right)\right)=\beta(\mathbf{x}) \boldsymbol{\sigma}\left(\nabla^{s} \overline{\mathbf{u}}, \nabla^{s} \dot{\overline{\mathbf{u}}}\right)
$$

Now define the stress in the intermediate layer as that calculated using the modified strain and strain rate functions

\section{Equation 49 - New Intermediate Stress}

$$
\tilde{\boldsymbol{\sigma}}^{\prime}=\boldsymbol{\sigma}\left(\hat{\boldsymbol{\varepsilon}}\left(\nabla^{s} \tilde{\mathbf{u}}\right), \dot{\hat{\boldsymbol{\varepsilon}}}\left(\nabla^{s} \dot{\tilde{\mathbf{u}}}\right)\right)
$$

Then Equation 41 is replaced by the same equation, but utilizing the the new intermediate stress

\section{Equation 50 - New Bielak Internal Forces}

$$
[F]=\left\{\begin{array}{l}
-\left.\int_{\Omega_{I I}}\left(\left(\boldsymbol{\sigma}-\tilde{\boldsymbol{\sigma}}^{\prime}\right) \bullet \nabla^{S}\left[\mathbf{e}_{i} N^{I}\right]+\rho(\ddot{\mathbf{u}}-\ddot{\tilde{\mathbf{u}}}) \bullet \mathbf{e}_{i} N^{I}\right) d \Omega\right|_{\text {node is not a member of } \Gamma^{b}} \\
-\left.\int_{\Omega_{I I}}\left(\left(\boldsymbol{\sigma}-\tilde{\boldsymbol{\sigma}}^{\prime}+\overline{\boldsymbol{\sigma}}\right) \bullet \nabla^{S}\left[\mathbf{e}_{i} N^{I}\right]+\rho(\ddot{\mathbf{u}}-\ddot{\tilde{\mathbf{u}}}+\ddot{\overline{\mathbf{u}}}) \bullet \mathbf{e}_{i} N^{I}\right) d \Omega\right|_{\text {node is a member of } \Gamma^{b}}
\end{array}\right.
$$

It is simple to verify, in analogy to Equation 42, that implementing Equation 50 leads to the following solution in the absence of the structure (no scattered waves)

Equation 51 - Solution for new implementation in the absence of the structure (no scattered waves)

$$
\mathbf{u}(x, t)=\left\{\begin{array}{c}
\overline{\mathbf{u}}(\mathbf{x}, t) \forall \mathbf{x} \in \Omega_{I} \\
\tilde{\mathbf{u}}(\mathbf{x}, t) \forall \mathbf{x} \in \Omega_{I I I} \\
\mathbf{0} \forall \mathbf{x} \in \Omega_{I I}
\end{array} \Rightarrow \boldsymbol{\sigma}(x, t)=\left\{\begin{array}{c}
\overline{\boldsymbol{\sigma}}(\mathbf{x}, t) \forall \mathbf{x} \in \Omega_{I} \\
\tilde{\boldsymbol{\sigma}}^{\prime}(\mathbf{x}, t) \forall \mathbf{x} \in \Omega_{I I I} \\
\mathbf{0} \forall \mathbf{x} \in \Omega_{I I}
\end{array}\right.\right.
$$

In the light of the discussion in Section 3.2.4, it is useful to suggest one more improvement to the method under consideration. Consider Figure 42 again. The outer region $\Omega_{I I}$ solely acts as an absorbing layer, and in reality extends to infinity. Hence, conceptually, this region satisfies the conceptual design of a discrete Perfectly Matched Layers, - a discretized, truncated perfectly matched medium ( [106] , see Figure 44. In general, the attenuation qualities of this region may be improved if the material dissipation is increased smoothly as the layer approaches the outer boundary, $\Gamma^{e}$. A direct time-domain implementation of such an approach has recently been investigated by Semblatt [100] . In this work the 
dissipation was increased simply by modifying the Rayleigh damping coefficients as a function of the distance from the inner boundary, with some success - more sophisticated dissipation functions as would be available if sophisticated soil models are used in $\Omega_{I I}$ and should naturally lead to even better results - a subject for future research.

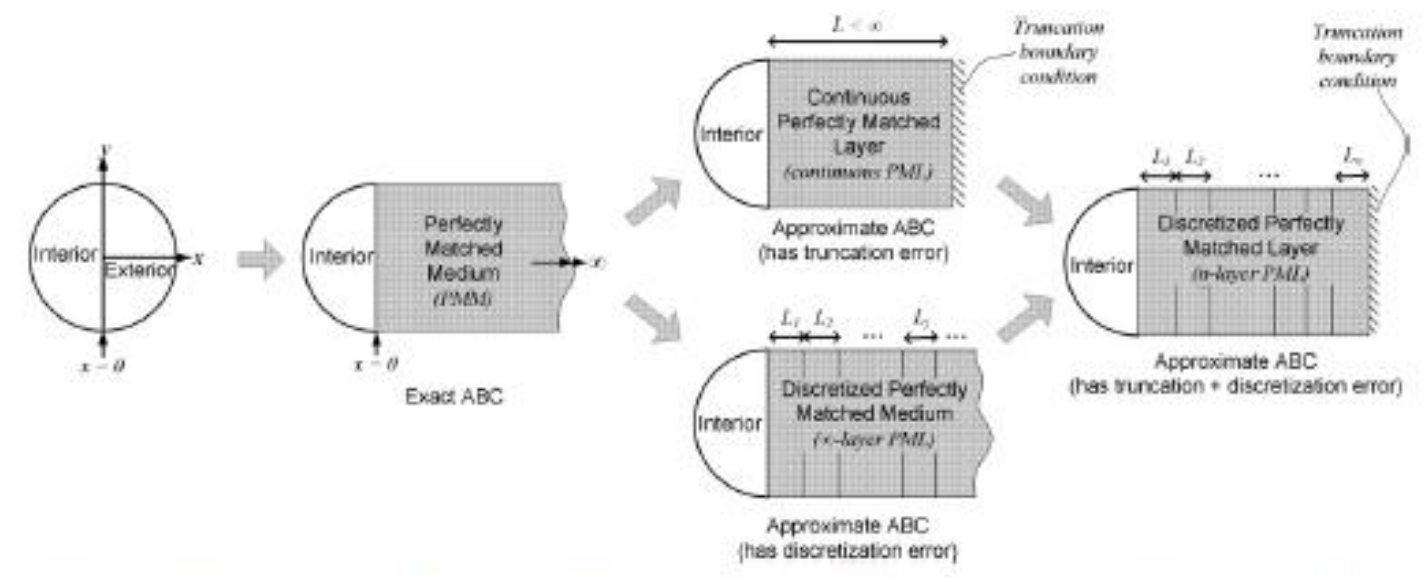

Fig. 5. The three steps in the design of a PML. hased local ABC; complex coordinate stretching truncation and discretization with truncation occurring before and after discretization.

Figure 44 - Perfectly Matched Layer as a discretized, truncated perfectly matched medium (Savadatti, 2012)

In summary, the formulations of Equation 41 and Equation 50 , with the scaling function defined by Equation 43 results in algorithms which:

1. Are valid for arbitrary non-linear materials

2. Can accommodate an arbitrarily-thick (more than one element) intermediate layer

The improved algorithm of Equation 50 also induces (in the absence of scattered waves) a stress and strain in the intermediate layer which

a. Smoothly approaches the exact solution for elements close to the inner boundary

b. Smoothly approaches the zero strain/zero stress solution for elements close to the boundary separating the intermediate layer and the outer region

Hence, the impedance within the intermediate layer varies naturally and smoothly between the highlystrained inner region and the undisturbed outer region. Therefore, scattered waves will be able to pass without reflection to the absorbing boundary.

Finally, as a subject for future research, the outer region $\Omega_{I I}$ may be thought of as a time-domain "perfectly matched layer", and the dissipative properties increased smoothly as a function of distance from the inner boundary, as in [100]. Such a treatment should lead to highly attenuated scattered waves reaching the outer boundary, thus further reducing the need for a high-performance absorbing boundary condition. 


\subsubsection{The Modified Bielak Method - Gravitational Preload and Contact}

The previous developments have assumed the baseline solution throughout the domain (that is, the solution without the earthquake input) to be the zero-displacement solution. In the nonlinear context, either because of pressure-dependent material models or nonlinear kinematics (contact), it is important to be able to account for a non-zero baseline solution, as would be present due to the effects of gravity. Let $\mathbf{u}^{g}(\mathbf{x})$ denote the non-zero baseline ("gravity") solution, calculated perhaps from an earlier quasistatic solution. Then assuming $\overline{\mathbf{u}}^{\prime}(\mathbf{x}, t)$ is the motion of the earthquake to be simulated, not including the gravity preload, either Equation 41 or Equation 50 can be implemented without change assuming

\section{Equation 52- Exact solution with gravity preload}

$$
\overline{\mathbf{u}}(\mathbf{x}, t)=\overline{\mathbf{u}}^{\prime}(\mathbf{x}, t)+\mathbf{u}^{g}(\mathbf{x})
$$

This would result in a zero-strain solution (in the absence of scattering waves) in the outer region $\Omega_{I I}{ }^{-}$ the gravity support is "built-in" to the seismic input along $\Gamma^{b}$. For sophisticated models which require non-zero compressive stress even at rest, this may not be desirable. In this case, one can additionally re-define the intermediate layer displacement of Equation 40 such that

Equation 53 - Scaled solution modified to accommodate non-zero static solution throughout the domain

$$
\tilde{\mathbf{u}}(\mathbf{x}, t)=\beta(\mathbf{x})\left(\overline{\mathbf{u}}(\mathbf{x}, t)-\mathbf{u}^{g}(\mathbf{x})\right)+\mathbf{u}^{g}(\mathbf{x})
$$

This also requires a change to Equation 46 , which become

\section{Equation 54 - Modified strain function accounting for the effects of gravity preload}

$$
\begin{aligned}
& \hat{\mathbf{\varepsilon}}\left(\nabla^{s} \mathbf{u}\right)=\nabla^{s} \mathbf{u}(\mathbf{x}, t)-\left(\left(\overline{\mathbf{u}}-\mathbf{u}^{g}\right) \otimes \nabla \beta\right)^{s} \\
& \dot{\hat{\boldsymbol{\varepsilon}}}\left(\nabla^{s} \dot{\mathbf{u}}\right)=\nabla^{s} \dot{\mathbf{u}}(\mathbf{x}, t)-(\dot{\overline{\mathbf{u}}} \otimes \nabla \beta)^{s}
\end{aligned}
$$

Then Equation 47 becomes

Equation 55 - Modified strain is proportional to exact solution strain plus gravity preload strain

$$
\mathbf{u}=\tilde{\mathbf{u}} \Rightarrow \hat{\boldsymbol{\varepsilon}}=\beta \nabla^{s} \overline{\mathbf{u}}^{\prime}+\nabla^{s} \mathbf{u}^{g}
$$

Then, assuming the analysis run is "restarted" from a quasi-static solution for the gravity problem, with the result saved as $\mathbf{u}^{g}(\mathbf{x})$, the solution for the seismic event can proceed as usual, with one caveat - at the outer boundary $\Gamma^{b}$ the reaction forces resulting from the quasi-static solution need to be applied the simplest solution (the one used here in DIABLO) is to save the reaction forces from the quasi-static solution, where $\Gamma^{b}$ is treated as a homogeneous Dirichlet (zero displacement) boundary condition. Another alternative would be to augment the absorbing boundary condition with boundary springs or quasi-static infinite elements, such as in [89] or [95]. 
Assuming the quasi-static gravity load is included, standard time-domain contact algorithms can be utilized. DIABLO features mortar-based contact algorithms. Historically, contact algorithms for generalpurpose time-domain analysis were based on "node-on-segment" algorithms, which enforce the contact as a collection of constraints - each constraint consists of a single node prohibited from penetrating into an opposing contact segment, enforced by a discrete nodal contact force. In contrast, in a "mortar" scheme the contact condition is formulated as a continuous constraint, parameterized by an interpolated contact pressure. The interpolated contact pressure acts as a smooth distributed lagrange multiplier, the "mortar" which acts to separate the surfaces. Mortar based algorithms are the current standard of the industry and were pioneered by Puso et. al. in the code NIKE3D [77].

In summary,

1. The incorporation of gravity preload is relatively trivial within the non-linear extension to the Bielak method.

2. If gravity preload is only desired within the interior region, practically no change is necessary to the algorithm.

3. If gravity preload is desired within the entire solution region, then

a. Minor adjustments are necessary to the formula for the modified strain function.

b. The external boundary requires the addition of either quasi-static forces (calculated and saved from the quasi-static solution) or boundary springs.

4. Assuming a solution with gravity preload, the use of contact algorithms for the soil/structure interface is trivial - existing algorithms can be used without modification. The current mortar contact algorithm utilized within DIABLO is the standard of the industry.

\subsubsection{The Modified Bielak Method - 3D input motions}

The non-linear Bielak method as expressed by Equation 41 or Equation 50 requires as its input the "exact solution" in terms of input motions. But it does not require the motions to have any particular direction or source. In the accompanying paper by Larry Hutchings [26], a technique is identified whereby a wave propagation code is used to provide these inputs as the result of earthquake waves traveling from a realistic source, located many kilometers away, see Figure 45 . The required input displacement, acceleration, and velocity is provided at regularly spaced points within and on the boundary of the intermediate layer $\Omega_{I I I}$, see Figure 46 . 


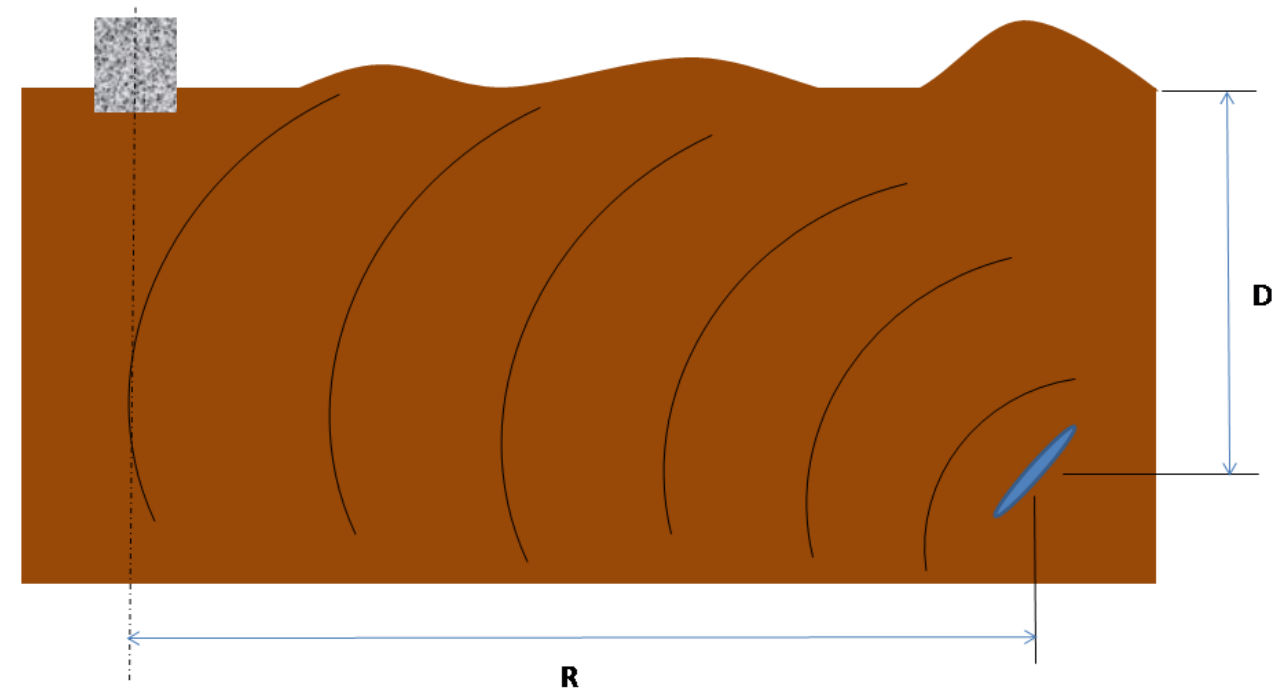

Figure 45- Source to Station

The earthquake intensity was synchronized with the data provided by Carl Costantino, mentioned earlier, at a single control point, "Point B" in Figure 46.

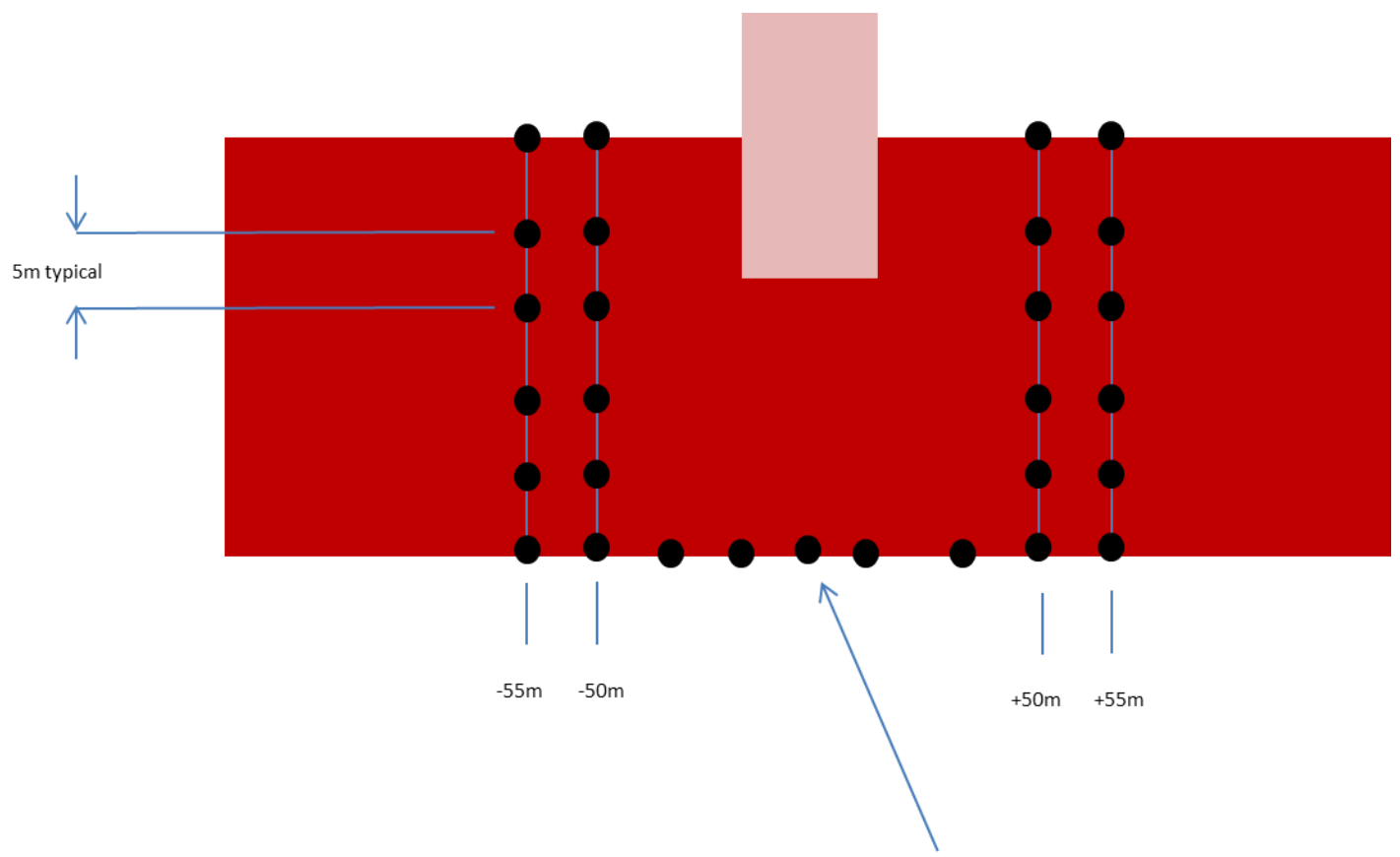

Point B

Figure 46 - Location of Data Points, and Calibration Point "B"

In comparison with the data from Carl Costantino, the provided motions are shorter in duration, with smaller peak spectra for the same $0.2 \mathrm{~g}$ earthquake. This may be a result of the Costantino data assuming "rock outcrop" motion at $\mathbf{3 7 0}$ feet whereas the Hutchings data assumed a different (but still stiff) soil profile below this depth, see Figure 47 and Figure 48 . The data infrastructure required to 
handle this input is currently being created within DIABLO so that, as part of future work, the SSI problem thus generated can be analyzed.

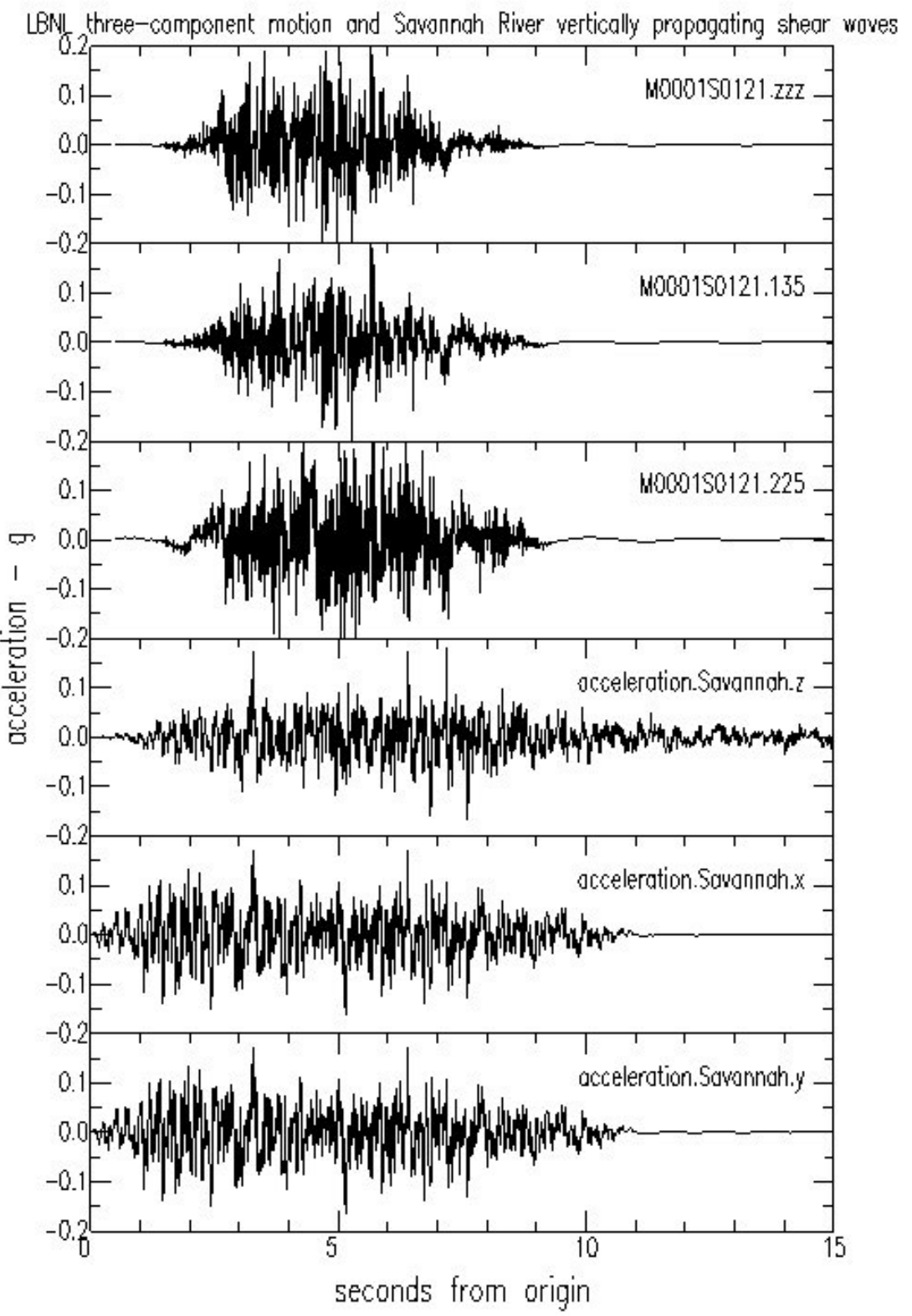

Figure 47 - Synthetic soil motions by Hutchings compared with Costantino data (Hutchings, 2012) 
LBNL three-component motion and Savannah River vertically propagating shear waves

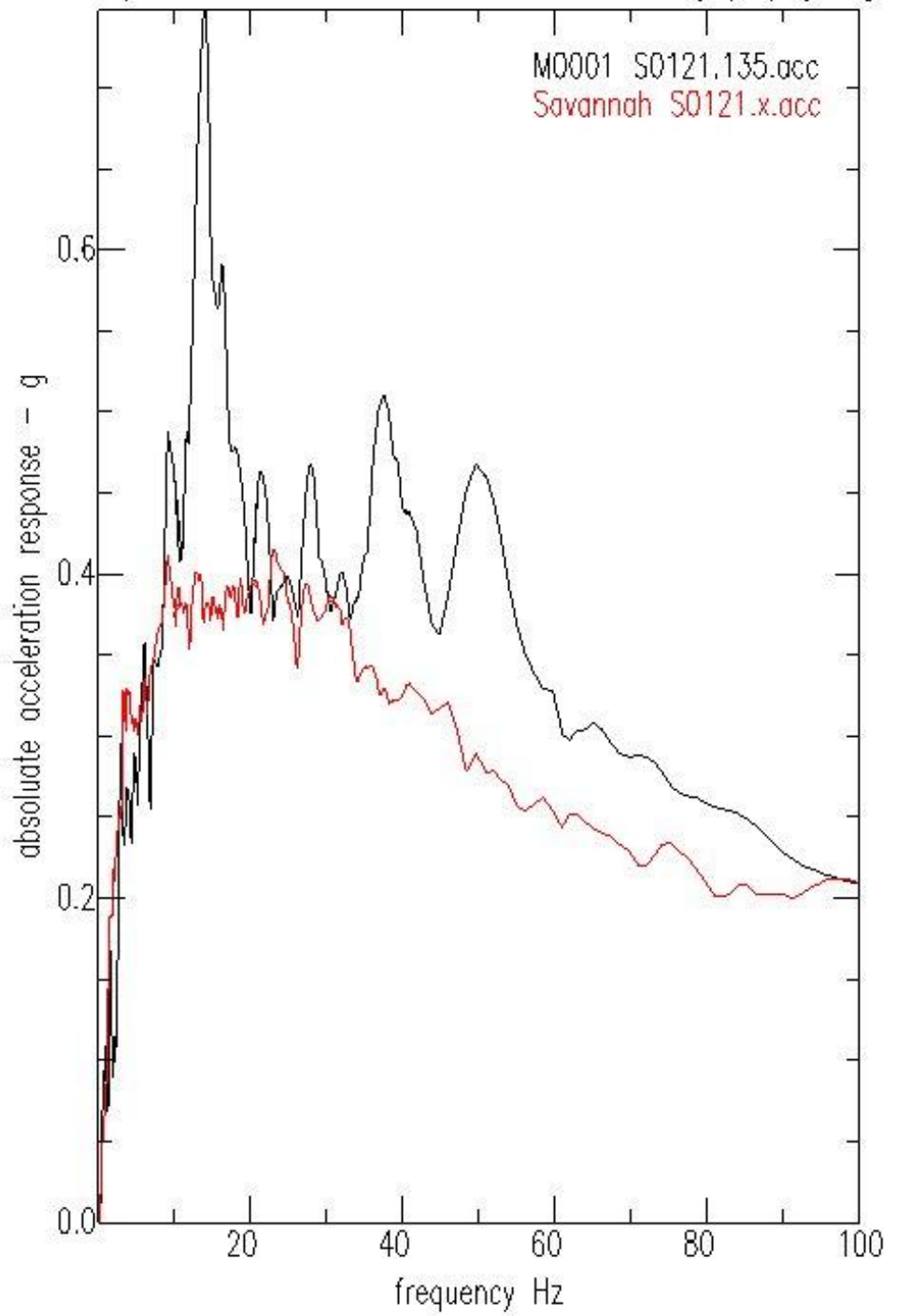

Figure 48 - Comparison of $X$ component acceleration spectra Hutchings Data versus Costantino data (Hutchings, 2012) at Point B

In summary,

1. The nonlinear Bielak method can accept 3D input data without modification.

2. A set of $3 D$ data representing a synthetic earthquake, propagated by its source, has been used to create input data for the Bielak Method.

3. The data has been calibrated for similar high-frequency peak spectra at a calibration point.

4. The data has shorter duration and lower peak spectra than the original data

5. The data-processing infrastructure is currently being created within DIABLO to use this data to directly model SSI for a 3D Input earthquake in the next phase of the current study. 


\subsubsection{The Modified Bielak Method - Summary}

1. The method of Bielak and coworkers has been extended to general non-linear materials.

2. The methodology allows for an intermediate region more than one element thick.

3. For a thick enough intermediate region, the method provides for impedance matching at both the inner and outer boundary of the intermediate region, with a moothly varying impedance within the region.

4. The method includes provisions for gravity preload and contact within the interior region.

5. The method allows for arbitrary input motions, as may be provided by a 3D wave propagation code synthesizing a motion directly from a source. Such a motion has been constructed and the infrastructure within DIABLO is being built to handle this data.

\subsection{Development of Input Motions}

\subsubsection{Derivation of 1-D input motions for typical earthquakes at the 0.05g, $0.2 \mathrm{~g}, 0.5 \mathrm{~g}$, and $0.9 \mathrm{~g}$ levels}

In order to determine the effect of soil-structure interaction (SSI) on the response of the reactor building structures and building supported systems and equipment, it is necessary to determine free-field motions on the boundary of the SSI model. In the SSI analysis method currently used in the nuclear industry, i.e., in the SASSI-based method, the free-field input motion is determined typically by assuming that: (a) seismic waves are vertically propagating shear waves for horizontal motion and vertically propagating compressional waves for vertical motion, and (b) the soil in the vicinity of the foundation consists of horizontal layers extending to infinity [29]. With these two assumptions, the "site response analysis," i.e., the determination of three-dimensional free-field input motion for SSI analyses reduces to a the analysis in one dimension to the response of a soil column to an acceleration spectrum based upon the probabilistic seismic hazard curve (a plot of peak acceleration versus selected design basis return period) and the Uniform Hazard Spectrum (UHS) defined for the particular location under study. Once the design basis return period is selected on the basis of the risk from a potential seismic failure of the reactor building structures or building supported systems and equipment, the corresponding peak acceleration is determined from the probabilistic hazard curve. This peak acceleration and the shape of the UHS define the input motion for the "site response analysis," typically in the form of a response spectrum at $5 \%$ damping. To perform the "site response analysis," the site soil column is then subjected to an acceleration time history input that is consistent with the $5 \%$ damped spectrum, and the input motion for the SSI analysis is determined.

Since the primary purpose of the current study is the determination of SSI effects using nonlinear timedomain analysis techniques and to compare these with those from SASSI, the development of seismic hazard curve and UHS was not included in this study. Instead, the existing bedrock acceleration timehistory for a DOE site was modified to develop a set of acceleration time-histories for four selected peak acceleration values: $0.05 \mathrm{~g}, 0.2 \mathrm{~g}, 0.5 \mathrm{~g}$, and $0.9 \mathrm{~g}$. These time histories were used to develop the input motions for the SSI analyses performed by BNL using SASSI and by LLNL using DIABLO. The time histories were developed by Professor Carl Costantino as part of his subcontract with LBNL. 


\subsection{Development of Material Models}

\subsubsection{Development Equivalent-Linear Material Coefficients using CARES for use in SASSI}

Carl Costantino, in collaboration with LBNL and BNL, took the soil profile defined in Table 2, Table 3, and Table 4, and ran CARES [18], [19] using the 1-D input motions as defined in Section 3.3.1 for the 0.2g, $0.5 \mathrm{~g}$, and $0.9 \mathrm{~g}$ cases. Equivalent-linear values of modulus and damping were calculated and reported in Figure 49 , Figure 50 , and Figure 51 . For the $0.2 \mathrm{~g}$ case, maximum strains are quite small (in the range of $0.02 \%$ ), but the equivalent-linear modulus averages about $80 \%$ of the maximum value, whereas damping is around $3-4 \%$. For the $0.9 \mathrm{~g}$ case, the strains are quite a bit larger, ranging up to $0.2 \%$, with damping values exceeding $14 \%$ in some cases, with the modulus degrading in some cases to just $30 \%$ of the maximum value.

\begin{tabular}{|c|c|c|c|c|c|c|c|c|c|c|c|c|c|}
\hline LAYER & LAYER & LAYER & LAYER & LAYER & UNIT & INITIAL & FINAL & EFFECT. & HYST: & FF SHEAR & EFFECT & TAU/ & $\mathrm{G} / \mathrm{Gmax}$ \\
\hline \multirow[t]{2}{*}{ NO. } & THICK & TOP & ВOTT & CENT & WGT & SHEAR & SHEAR & SHEAR & DAMP & STRESS & VERBRDN & SIGMA & \\
\hline & $(\mathrm{ft})$ & $(\mathrm{ft})$ & $(\mathrm{ft})$ & $(\mathrm{ft})$ & (kcf) & $\operatorname{VEL}(f p s)$ & $\operatorname{VEL}(f p s)$ & STR (\%) & $(\%)$ & (ksf) & (ksf) & & \\
\hline 1 & 30 & 0 & 30 & 15 & 0.125 & 1580 & 1484.6 & $3.63 \mathrm{E}-03$ & 3.04 & 0.31 & 1.872 & 0.165 & 0.883 \\
\hline 2 & 44 & 30 & 74 & 52 & 0.128 & 1240 & 1087.8 & $1.74 \mathrm{E}-02$ & 4.14 & 0.819 & 6.558 & 0.125 & 0.77 \\
\hline 3 & 49 & 74 & 123 & 98.5 & 0.125 & 1200 & 986.2 & $2.98 \mathrm{E}-02$ & 5.37 & 1.124 & 12.429 & 0.09 & 0.675 \\
\hline 4 & 67 & 123 & 190 & 156.5 & 0.123 & 1564 & 1403.4 & $1.87 \mathrm{E}-02$ & 3.46 & 1.405 & 19.607 & 0.072 & 0.805 \\
\hline 5 & 15 & 190 & 205 & 197.5 & 0.132 & 2153 & 2047.2 & $9.22 \mathrm{E}-03$ & 2.18 & 1.581 & 24.716 & 0.064 & 0.904 \\
\hline 6 & 47 & 205 & 252 & 228.5 & 0.132 & 1720 & 1561.4 & $1.65 \mathrm{E}-02$ & 3.21 & 1.645 & 28.805 & 0.057 & 0.824 \\
\hline 7 & 26 & 252 & 278 & 265 & 0.126 & 1415 & 1260.4 & $2.72 \mathrm{E}-02$ & 3.34 & 1.692 & 33.545 & 0.05 & 0.793 \\
\hline 8 & 25 & 278 & 303 & 290.5 & 0.132 & 1675 & 1539.9 & $1.82 \mathrm{E}-02$ & 2.74 & 1.769 & 36.83 & 0.048 & 0.845 \\
\hline 9 & 67 & 303 & 370 & 336.5 & 0.131 & 2015 & 1891.4 & $1.38 \mathrm{E}-02$ & 2.33 & 2.012 & 42.864 & 0.047 & 0.881 \\
\hline \multirow[t]{2}{*}{ rock } & & 370 & & & 0.15 & 11000 & & & 1 & & & & \\
\hline & & & & & & & & & & & & & \\
\hline
\end{tabular}

Figure 49 - CARES-derived equivalent-linear modulus and damping for $0.2 \mathrm{~g}$ case

\begin{tabular}{|c|c|c|c|c|c|c|c|c|c|c|c|c|c|}
\hline LAYER & LAYER & LAYER & LAYER & LAYER & UNIT & INITIAL & FINAL & EFFECT. & HYST & EFF SHEA & EFFECT & TAU/ & G/Gmax \\
\hline NO. & THICK & TOP & BOTT & CENT & WGT & SHEAR & SHEAR & SHEAR & DAMP & STRESS & OVERBRD & SIGMA & \\
\hline & $(\mathrm{ft})$ & $(\mathrm{ft})$ & $(\mathrm{ft})$ & $(\mathrm{ft})$ & (kcf) & $\operatorname{VEL}(f p s)$ & VEL(fps) & STR (\%) & $(\%)$ & (ksf) & (ksf) & & \\
\hline 1 & 30 & 0 & 30 & 15 & 0.125 & 1580 & 1368.4 & $8.96 \mathrm{E}-03$ & 4.88 & 0.65 & 1.872 & 0.347 & 0.75 \\
\hline 2 & 44 & 30 & 74 & 52 & 0.128 & 1240 & 930.5 & $5.18 \mathrm{E}-02$ & 7.61 & 1.78 & 6.558 & 0.271 & 0.563 \\
\hline 3 & 49 & 74 & 123 & 98.5 & 0.125 & 1200 & 785 & $9.96 \mathrm{E}-02$ & 10.39 & 2.378 & 12.429 & 0.191 & 0.428 \\
\hline 4 & 67 & 123 & 190 & 156.5 & 0.123 & 1564 & 1243.3 & $5.02 \mathrm{E}-02$ & 6.17 & 2.966 & 19.607 & 0.151 & 0.632 \\
\hline 5 & 15 & 190 & 205 & 197.5 & 0.132 & 2153 & 1893.5 & $2.31 \mathrm{E}-02$ & 3.87 & 3.381 & 24.716 & 0.137 & 0.773 \\
\hline 6 & 47 & 205 & 252 & 228.5 & 0.132 & 1720 & 1376.7 & $4.81 \mathrm{E}-02$ & 6.01 & 3.739 & 28.805 & 0.13 & 0.641 \\
\hline 7 & 26 & 252 & 278 & 265 & 0.126 & 1415 & 1074.7 & $9.00 \mathrm{E}-02$ & 6.81 & 4.068 & 33.545 & 0.121 & 0.577 \\
\hline 8 & 25 & 278 & 303 & 290.5 & 0.132 & 1675 & 1368.4 & 5.57E-02 & 5.32 & 4.263 & 36.83 & 0.116 & 0.667 \\
\hline 9 & 67 & 303 & 370 & 336.5 & 0.131 & 2015 & 1756.7 & $3.41 \mathrm{E}-02$ & 3.8 & 4.275 & 42.864 & 0.1 & 0.76 \\
\hline rock & & 370 & & & 0.15 & 11000 & & & 1 & & & & \\
\hline
\end{tabular}

Figure 50 - CARES-derived equivalent-linear modulus and damping for $0.5 \mathrm{~g}$ case 


\begin{tabular}{|c|c|c|c|c|c|c|c|c|c|c|c|c|c|}
\hline LAYER & LAYER & LAYER & LAYER & LAYER & UNIT & INITIAL & FINAL & EFFECT. & HYST & EFF SHEAA & EFFECT & TAU/ & G/Gmax \\
\hline NO. & THICK & TOP & BOTT & CENT & WGT & SHEAR & SHEAR & SHEAR & DAMP & STRESS & OVERBRD & SIGMA & \\
\hline & $(\mathrm{ft})$ & $(\mathrm{ft})$ & (ft) & $(\mathrm{ft})$ & (kcf) & VEL(fps) & VEL(fps) & STR (\%) & $(\%)$ & (ksf) & (ksf) & & \\
\hline 1 & 30 & 0 & 30 & 15 & 0.125 & 1580 & 1263 & $1.56 \mathrm{E}-02$ & 6.76 & 0.964 & 1.872 & 0.515 & 0.639 \\
\hline 2 & 44 & 30 & 74 & 52 & 0.128 & 1240 & 808.6 & $1.01 \mathrm{E}-01$ & 10.46 & 2.621 & 6.558 & 0.4 & 0.425 \\
\hline 3 & 49 & 74 & 123 & 98.5 & 0.125 & 1200 & 647.3 & 2.06E-01 & 14.57 & 3.342 & 12.429 & 0.269 & 0.291 \\
\hline 4 & 67 & 123 & 190 & 156.5 & 0.123 & 1564 & 1130.9 & $8.59 \mathrm{E}-02$ & 8.12 & 4.198 & 19.607 & 0.214 & 0.523 \\
\hline 5 & 15 & 190 & 205 & 197.5 & 0.132 & 2153 & 1794.7 & $3.69 \mathrm{E}-02$ & 5.05 & 4.856 & 24.716 & 0.196 & 0.695 \\
\hline 6 & 47 & 205 & 252 & 228.5 & 0.132 & 1720 & 1269.7 & $7.71 \mathrm{E}-02$ & 7.72 & 5.093 & 28.805 & 0.177 & 0.545 \\
\hline 7 & 26 & 252 & 278 & 265 & 0.126 & 1415 & 998.7 & $1.32 \mathrm{E}-01$ & 8.63 & 5.164 & 33.545 & 0.154 & 0.498 \\
\hline 8 & 25 & 278 & 303 & 290.5 & 0.132 & 1675 & 1302 & $7.78 \mathrm{E}-02$ & 6.36 & 5.397 & 36.83 & 0.147 & 0.604 \\
\hline 9 & 67 & 303 & 370 & 336.5 & 0.131 & 2015 & 1662.3 & $5.19 \mathrm{E}-02$ & 5.11 & 5.836 & 42.864 & 0.136 & 0.681 \\
\hline rock & & 370 & & & 0.15 & 11000 & & & 1 & & & & \\
\hline
\end{tabular}

Figure 51 - CARES-derived equivalent-linear modulus and damping for the $0.9 \mathrm{~g}$ case

\subsubsection{Development of Ramberg-Osgood (RO) coefficients using RAMBO for use in DIABLO}

The code RAMBO was used to fit the EPRI data as tabulated in Table 3 and Table 4 . The result of the fit is compiled in Figure 52. Graphical representations of the fits are provided as Figure 53, Figure 54, and Figure 55. Inspection of the fits indicates that moduli matched very well, except at strains over about $1 \%$, where the experimental data appears to have been truncated. Damping data also matches very well below about $1 \%$ strain and above zero strain. At zero to very small strains the R-O model has essentially zero damping, whereas the EPRI data has non-zero damping even at $0 \%$ strain. For our simple comparison, the Ramberg-Osgood model will be augmented with a small amount of Rayleigh (viscous) damping, which will serve to provide damping at the small strain levels - see Section 3.4.3 for a more complete discussion of the inclusion of Rayleigh Damping.

\begin{tabular}{|r|r|r|r|r|r|r|r|}
\hline Soil layer & alpha & \multicolumn{1}{l|}{ R } & Reference str & Reference stres & Gmax (Gpa) & Bulk Modulus (Gpa & density (kg/m^3) \\
\hline $\mathbf{1}$ & 1.060 & 2.437 & $1.456 \mathrm{E}-04$ & 67568.62 & 0.464 & 1.006 & 2002.25 \\
\hline $\mathbf{2}$ & 1.065 & 2.254 & $3.778 \mathrm{E}-04$ & 110660.85 & 0.293 & 0.635 & 2050.30 \\
\hline $\mathbf{3}$ & 1.063 & 2.253 & $3.787 \mathrm{E}-04$ & 101421.88 & 0.268 & 0.580 & 2002.25 \\
\hline $\mathbf{4}$ & 1.076 & 2.210 & $5.774 \mathrm{E}-04$ & 258484.45 & 0.448 & 0.970 & 1970.21 \\
\hline $\mathbf{5}$ & 1.076 & 2.210 & $5.770 \mathrm{E}-04$ & 525725.24 & 0.911 & 1.973 & 2114.38 \\
\hline $\mathbf{6}$ & 1.076 & 2.210 & $5.774 \mathrm{E}-04$ & 335498.89 & 0.581 & 1.259 & 2114.38 \\
\hline $\mathbf{7}$ & 1.075 & 2.159 & $8.694 \mathrm{E}-04$ & 326397.81 & 0.375 & 0.813 & 2018.27 \\
\hline $\mathbf{8}$ & 1.075 & 2.159 & $8.694 \mathrm{E}-04$ & 479116.68 & 0.551 & 1.194 & 2114.38 \\
\hline $\mathbf{9}$ & 1.075 & 2.159 & $8.694 \mathrm{E}-04$ & 688096.78 & 0.792 & 1.715 & 2098.36 \\
\hline
\end{tabular}

Figure 52 - Result of Ramberg-Osgood Fit using RAMBO to EPRI data for the 9 Soil Layers 

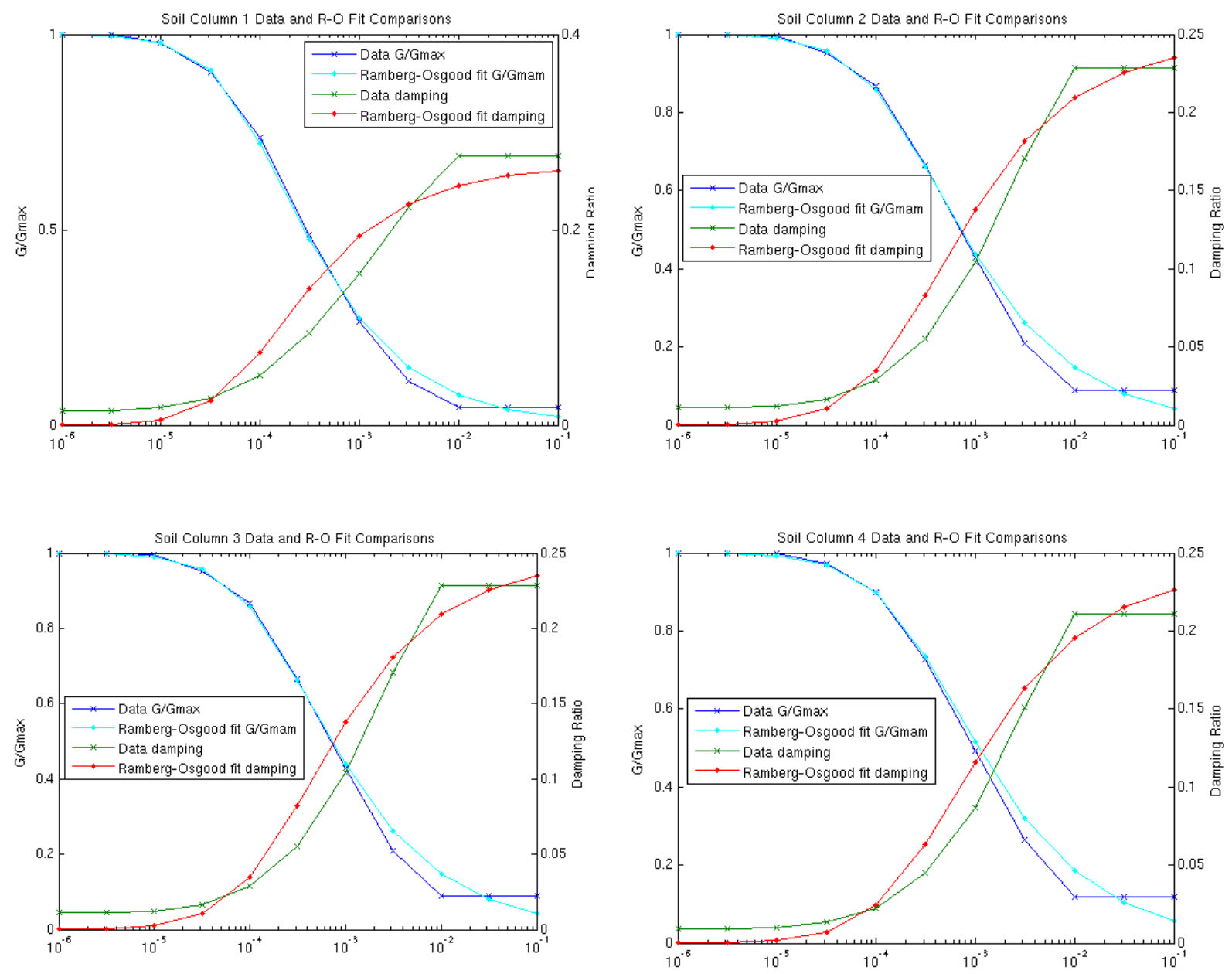

Figure 53 - Graphical Representation of Ramberg-Osgood Fits to Soil Layers 1-4 

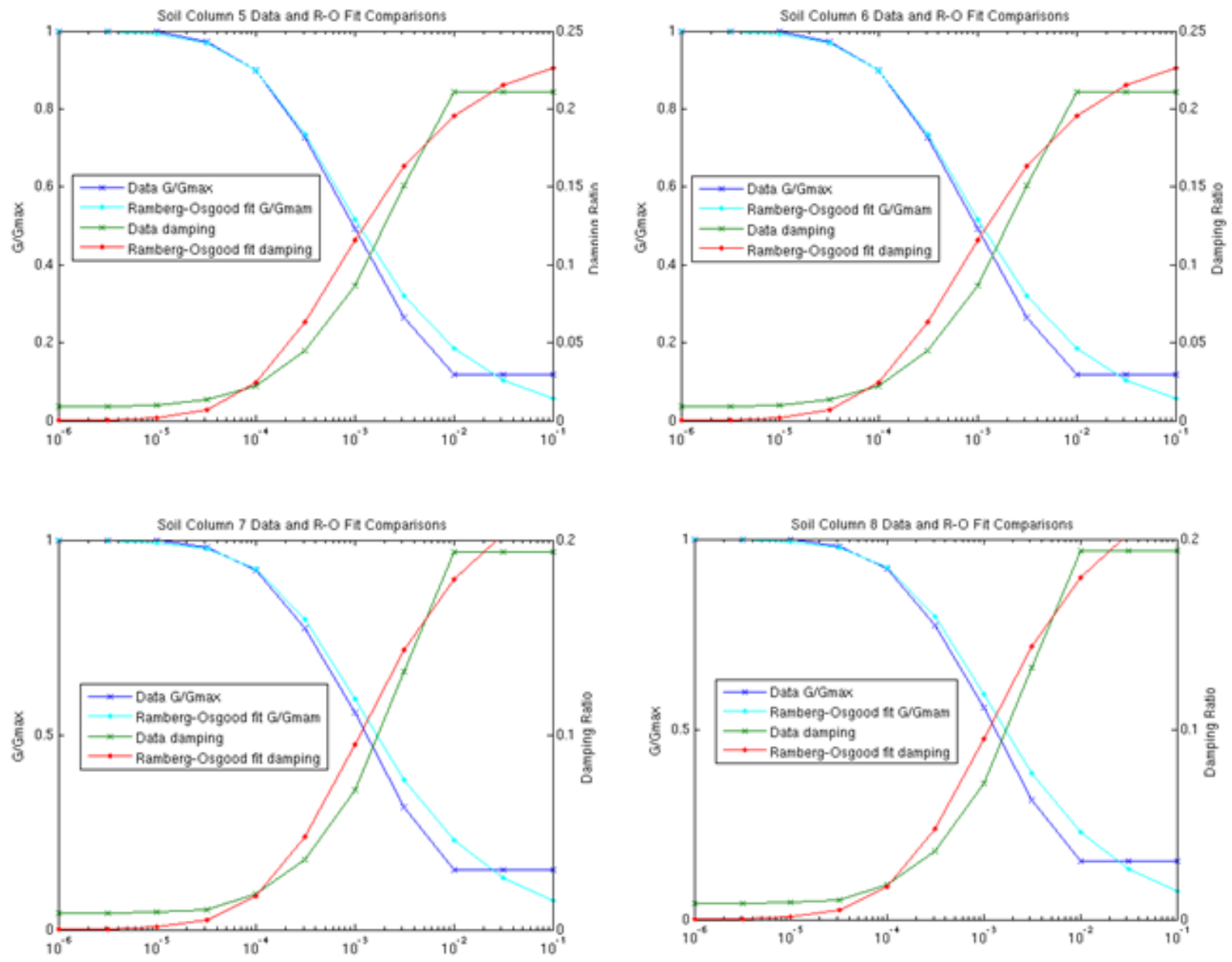

Figure 54 - Graphical Representation of Ramberg-Osgood Fits to Soil Layers 5-8

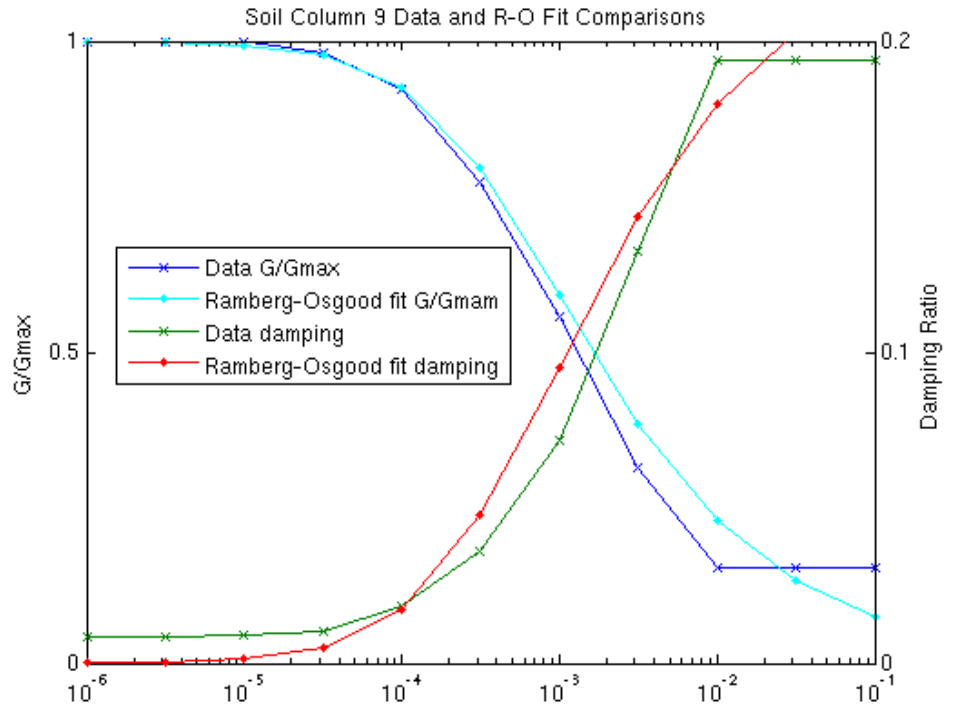

Figure 55 - Graphical Representation of Ramberg-Osgood Fits for Soil Layer 9 


\subsubsection{Comparison of 1D Vertical Soil Column Response - CARES Using Equivalent Linear Properties versus DIABLO using Ramberg-Osgood Model}

The results of applying the in-column ${ }^{16}$ motion to the base of a 1-D soil column using DIABLO was compared to the equivalent CARES [18], [19] analysis for $0.2 \mathrm{~g}, 0.5 \mathrm{~g}$, and $0.9 \mathrm{~g}$ earthquakes modeled as vertically propagating shear waves. The initial comparisons were performed without any viscous damping for the Ramberg-Osgood model. The results of this comparison for the $0.2 \mathrm{~g}$ case are reported via $5 \%$ damped response spectra ${ }^{17}$, in Figure 56 through Figure 58. Typically, the response is reasonable at lower frequencies, and generally DIABLO has significantly greater response at high frequencies. There are some peaks even at low frequencies for which DIABLO produces higher response.
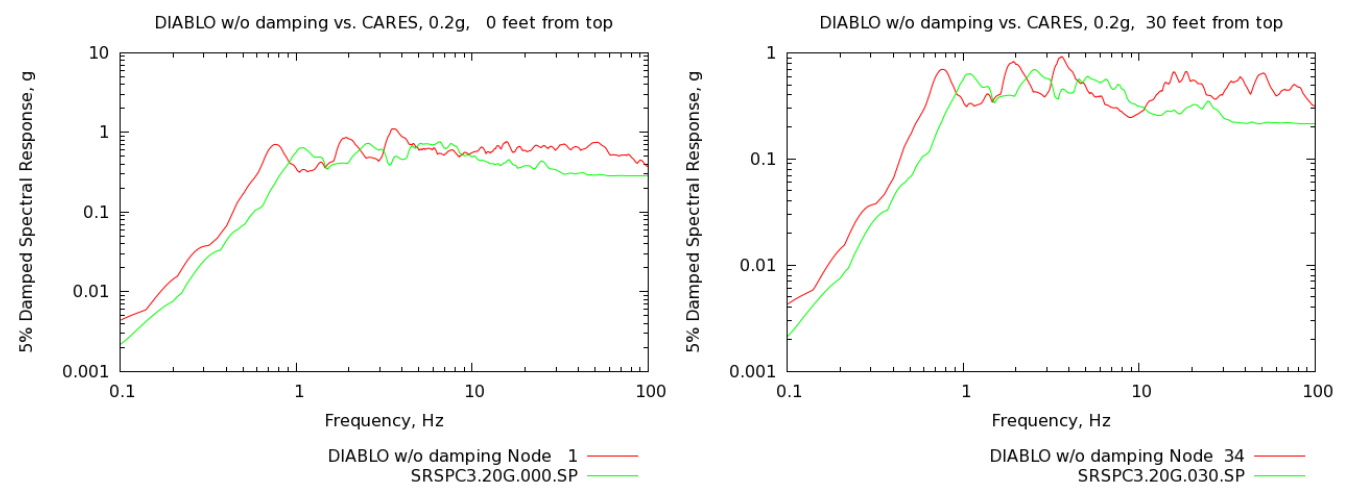

Figure 56 -1D Soil Column, 0.2g earthquake, 5\% Response Spectra: DIABLO w/o viscous damping (red) versus CARES (green), distance 0 and $30 \mathrm{ft}$ from top surface
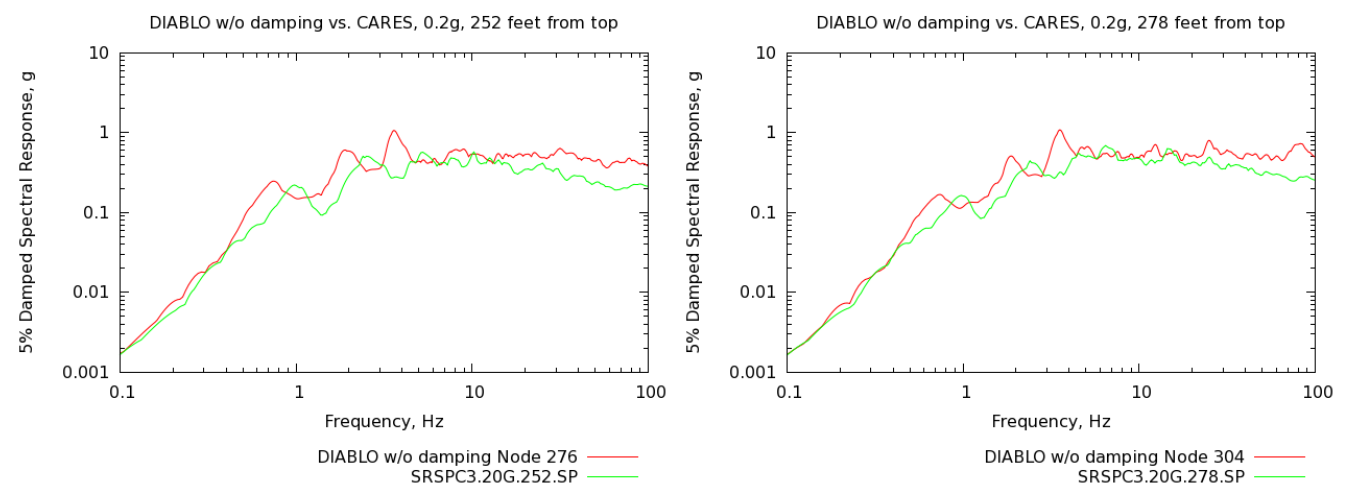

Figure 57 - 1D Soil Column, 0.2g earthquake, 5\% Response Spectra: DIABLO w/o viscous damping (red) versus CARES (green) 1-d with no viscous damping, distance 252 and 278 feet from top surface

\footnotetext{
16 See Section 3.2.4.1, and specifically the discussion around Figure 37

${ }^{17}$ Response of a harmonic oscillator with $5 \%$ of critical damping to an acceleration time history, as a function of the oscillator natural frequency - see for example [113].
} 

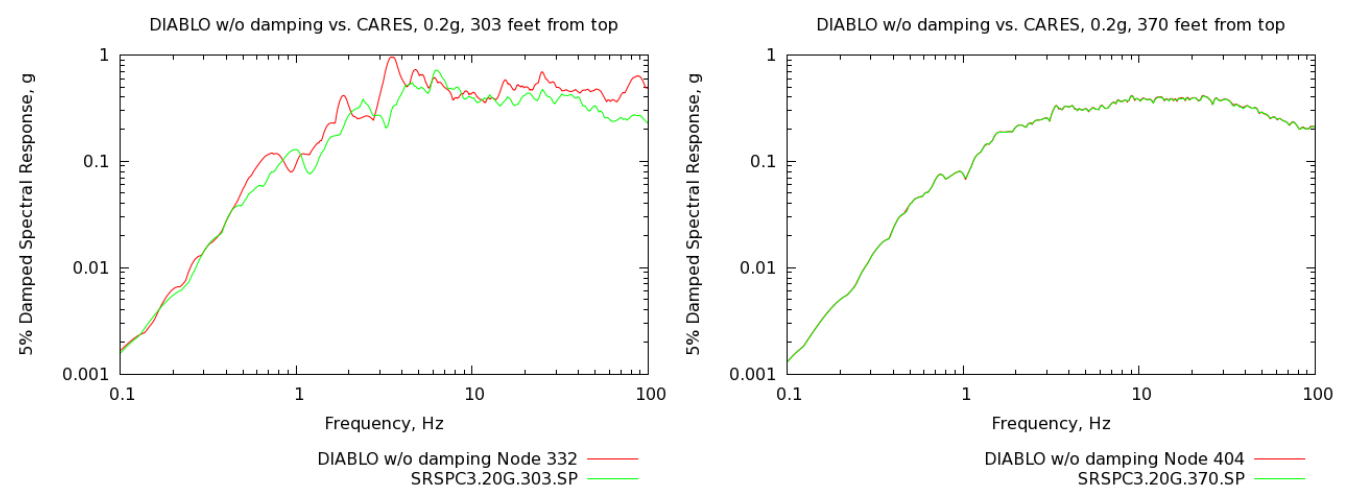

Figure 58 - 1D Soil column, $0.2 \mathrm{~g}$ earthquake, 5\% Response Spectra: DIABLO w/o viscous damping(red) versus CARES 1-d distance 303 and $370 \mathrm{ft}$ from top surface

The complete data set for the $0.2 \mathrm{~g}$ case is provided in the Appendix A.1. Recognizing that a significant source of error was the inability of the Ramberg-Osgood model to provide damping at low strain levels (see, for example, Figure 53), it was decided that a small amount of viscous damping was needed. This was implemented by utilizing Rayleigh damping with damping ratio $\xi=0.015$ for systems with natural frequencies $1 \mathrm{~Hz}$ and $30 \mathrm{~Hz}$, see Equation 20. The resulting Rayleigh damping values, applied uniformly for all the soil layers, are

Equation 56 - Rayleigh Damping Parameters used in conjunction with Ramberg-Osgood Soil Model, for 1.5\% damping at 1 and $30 \mathrm{~Hz}$.

$$
\begin{aligned}
& \alpha_{R}=0.1824 \\
& \beta_{R}=0.0001524
\end{aligned}
$$

The result of the addition of damping was significantly better match, especially at the higher frequencies, see Figure 59, Figure 60, and Figure 61. The complete data set is presented in the Appendix A.2. Similar comments apply for the behavior at $0.5 \mathrm{~g}$ and $0.9 \mathrm{~g}$, the complete data sets are provided in the Appendices A.3 and A.4.

In summary, the combination of a Ramberg-Osgood model with a small amount viscous damping ${ }^{18}$, which primarily serves to quite high-frequency/low-amplitude modes, provides a good match to the "equivalent linear" model with hysteretic damping. Note that the Ramberg-Osgood model coefficients are only fit once - they do not change with the earthquake level, as in the equivalent-linear method.

\footnotetext{
18 Physically, a small amount of viscous damping is justifiable - as it represents the effect of fluid flow in the pores, see [25]
} 

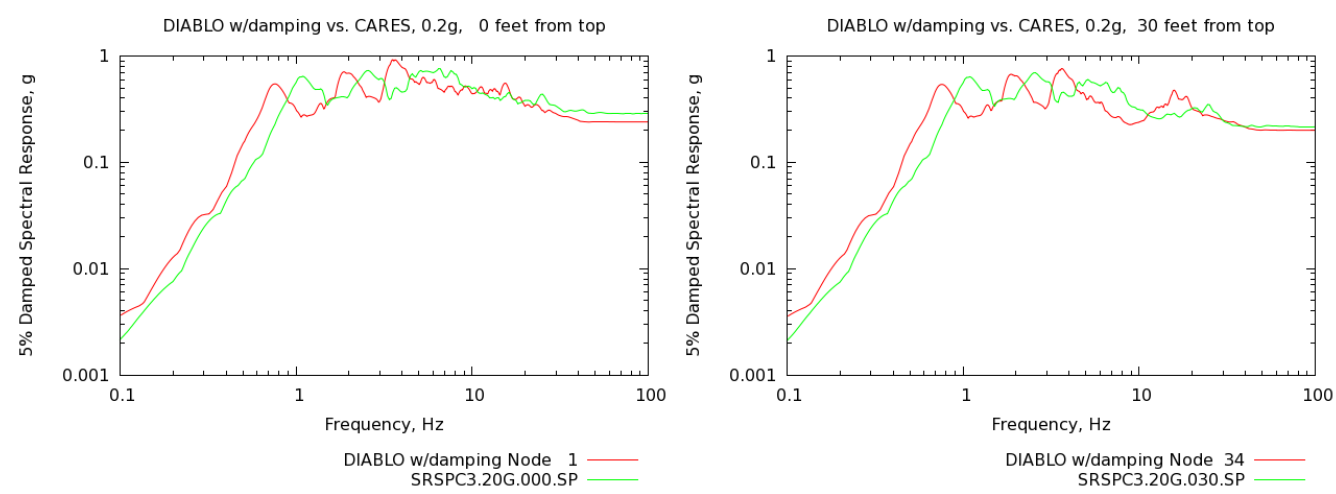

Figure 59 - 1D Soil column, $0.2 \mathrm{~g}$ earthquake, 5\% Response Spectra: DIABLO with viscous damping(red) versus CARES 1-d distance 0 and $30 \mathrm{ft}$ from top surface
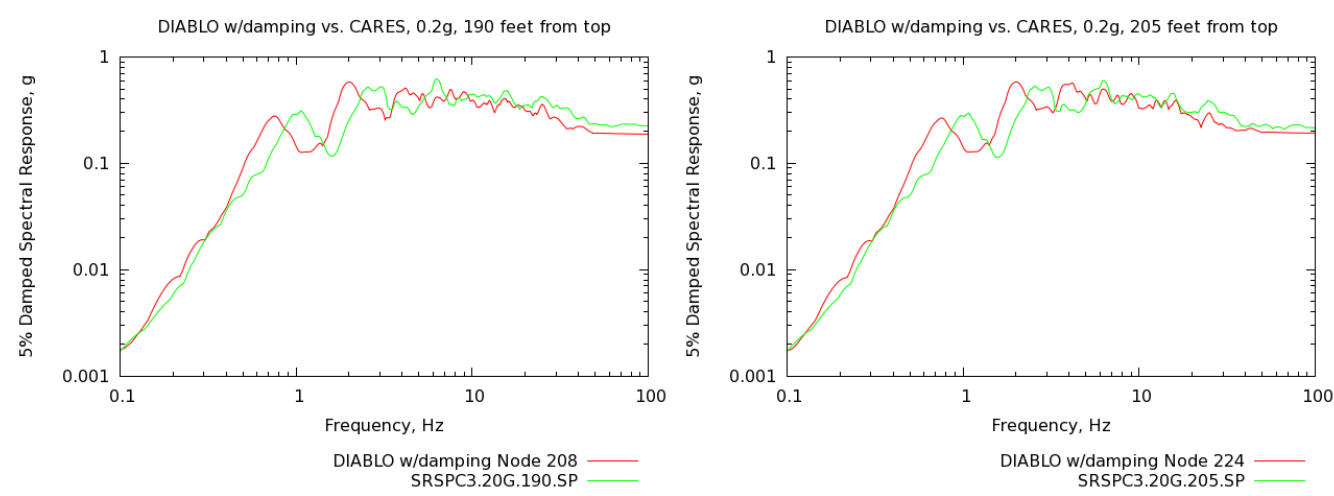

Figure 60 - 1D Soil column, 0.2g earthquake, 5\% Response Spectra: DIABLO with viscous damping(red) versus CARES 1-d distance 190 and $205 \mathrm{ft}$ from top surface
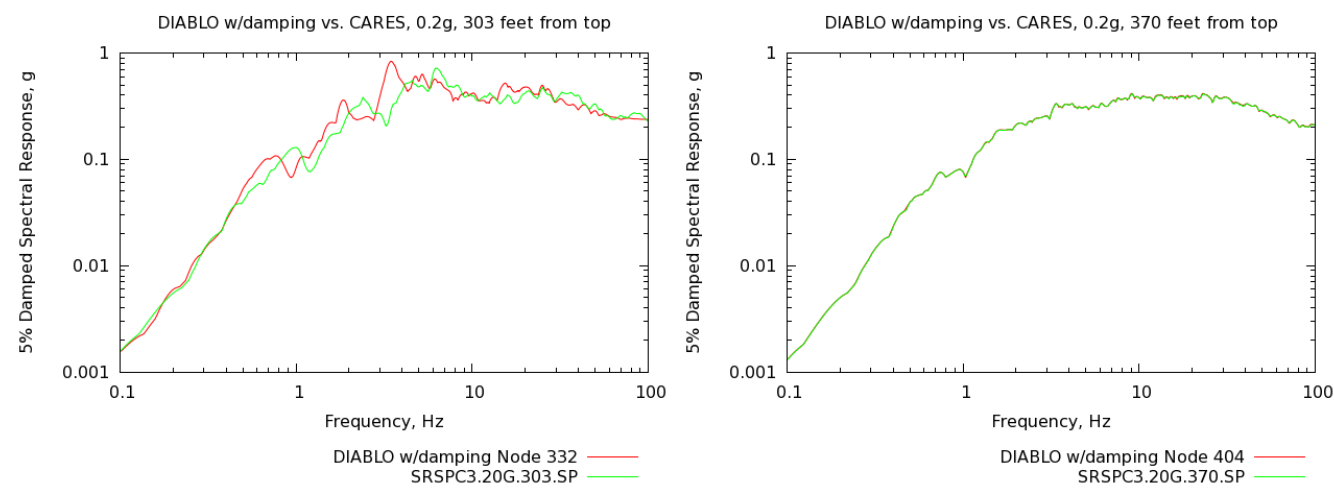

Figure 61 - 1D Soil column, 0.2g earthquake, 5\% Response Spectra: DIABLO with viscous damping(red) versus CARES 1-d distance 303 and $370 \mathrm{ft}$ from top surface 


\subsubsection{Development of advanced soil models with traceability to EPRI test data.}

The report by Pisano and Jeremic [25] , produced as part of this project, aims to develop 3-dimensional soil models based upon the modern framework of computational plasticity, which are traceable to EPRI test data. The work is based on a hierarchy of models of increasing complexity, beginning with the investigation of the hysteretic behavior (with the possibility of including viscous damping as well) of two models of increasing complexity

- Von Mises assuming Perfect Plasticity (no hardening)

- Drucket-Prager with Kinematic Hardening

The Von Mises/Perfectly Plastic model is perhaps the simplest (in the context of computational plasticity) that can be applied to soils. It has been used to predict the behavior of undrained soils, especially clays. It is defined by an elastic response model, (typically linear elasticity), effective when operating below the yield point, and a yield criteria formulated in terms of the deviatoric stress tensor $\mathbf{s}$ (see also Equation 36)

\section{Equation 57- Von Mises Yield Criteria (see Pisano, et. al., equation 2.1)}

$$
f=\sqrt{\frac{3}{2}\|\mathbf{S}\|^{2}-\sigma_{0}}
$$

The yield strength $\sigma_{0}$ corresponds to the undrained strength. Perfect plasticity with an associative flow rule is assumed, which because yield stress is formulated solely in terms of the deviatoric stress can be written equivalently either in total stress/strain space or in terms of deviatoric stress/strain components

Equation 58 - Von Mises Flow Rule

$$
\dot{\boldsymbol{\varepsilon}}_{p}=\dot{\lambda} \frac{\partial f}{\partial \boldsymbol{\sigma}} \Leftrightarrow \dot{\mathbf{e}}_{p}=\dot{\lambda} \frac{\partial f}{\partial \mathbf{s}}
$$

Because of the assumption of perfect plasticity, at yield the material has effectively no strength as long as the strain rate is still in the direction of loading.

Given an undrained strength $\sigma_{0}$, the limiting shear stress can be determined as

Equation 59 - Limiting shear stress (Pisano, et. al, 2012, equation 2.13)

$$
\tau_{s u}=\frac{2}{\sqrt{3}} \sigma_{0}
$$

Then the effective shear modulus can be derived as a function of the maximum strain 
Equation 60 - Shear Modulus Degradation Relationship for Von Mises with Perfect Plasticity (Pisano, et. al., 2012, equation 2.14)

$$
\frac{G}{G_{\max }}=\min \left(\frac{\tau_{s u}}{G_{\max } \gamma_{\max }}, 1\right)
$$

Similar relationships can be derived for an effective damping coefficient. Because of the perfect plasticity assumption, it is difficult to match both damping and shear modulus degradation - since effectively a single parameter, the undrained strength, is being used to fit both curves. In addition, Equation 60 does not fit the data very well - for values of $\gamma_{\max }$ sufficiently small (such that $G_{\max } \gamma_{\max }<$ $\tau_{s u}$ ) the shear modulus does not degrade whatsoever. In order to match damping data with any degree of accuracy it is necessary to add a significant amount of viscous dissipation - which introduces the usual difficulties of rate-(i.e. frequency) dependence - an example of this is seen in Figure 62, where it proves impossible to produce varying damping curves without also varying significantly the shear modulus degradation. Notice also that the shear modulus does not degrade whatsoever below a value which is dependent on the undrained shear strength.
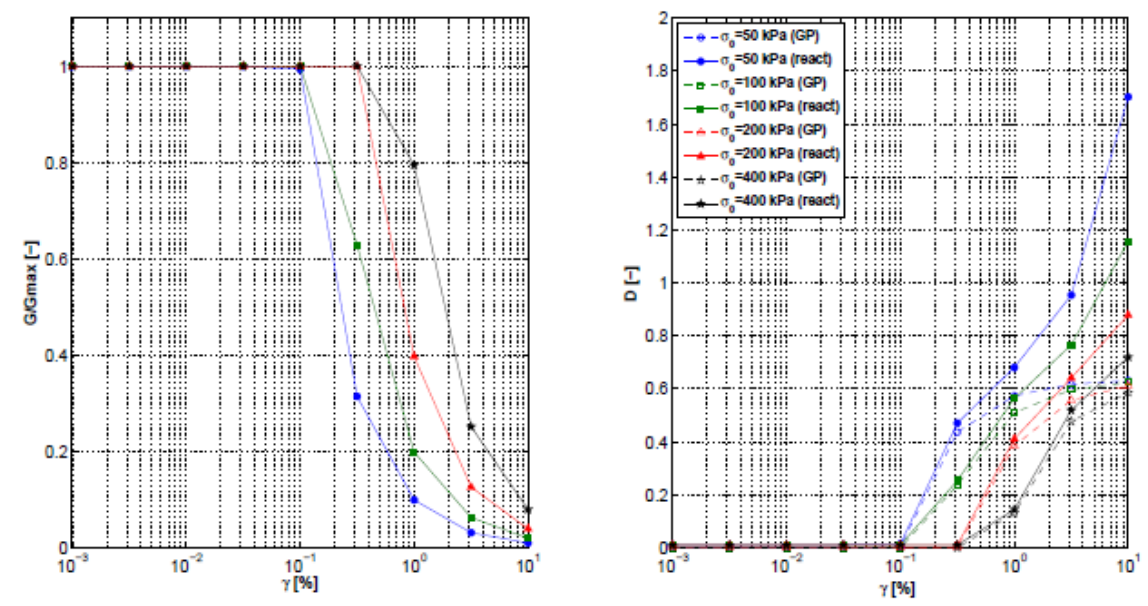

Figure 62 - Effective of varying the undrained strength for the Von Mises/Perfectly Plastic Model on the modulus degradation and damping ratios, assuming $2 \%$ viscous damping - see Figure 2.11 of (Pisano, et. al., 2012)

One step towards greater accuracy is accomplished by utilizing a material model with hardening. In the Von Mises/Perfectly Plastic model, the material has essentially no stiffness once the undrained strength is reached - but obeys perfect elasticity up to that point. Hence below the undrained strength the material has essentially no dissipation (beyond that provided by viscous damping), and above that point the material has essentially no stress response. By providing the material with a hardening law, the yield point can be brought essentially to the origin, thus providing plastic dissipation almost at zero strain, yet still provide stress response at non-zero strains. The form of the hardening and flow rules then govern the evolution of the stress response (the modulus degradation) and dissipation. The authors investigate just such a model, the Drucker-Prager plasticity model utilizing the ArmstrongFrederick Kinematic Hardening model (DPKH). Letting $p$ be the isochoric pressure and $\boldsymbol{\alpha}$ the back stress, the DPKH yield function may be written as 
Equation 61 - DPKH yield function - see Equation 2.2 of (Pisano, et. al., 2012)

$$
f=\sqrt{\|\mathbf{s}-p \boldsymbol{\alpha}\|^{2}}-\sqrt{\frac{2}{3}} k p
$$

Then the flow rule is non-associative

Equation 62 - DPKH flow rule - see Equations 2.3 and 2.4 of (Pisano, et. al, 2012)

$$
\dot{\boldsymbol{\varepsilon}}_{p}=\dot{\lambda}\left[\left(\frac{\partial f}{\partial \boldsymbol{\sigma}}\right)^{d e v}-\frac{1}{3} D \mathbf{I}\right]
$$

With $D$ defined via an auxiliary relationship

Equation 63 - DPKH Dilatancy parameter - see Equation 2.5 of (Pisano, et. al., 2012)

$$
D=\xi\left(\sqrt{\frac{2}{3}} k_{d}-\|\mathbf{r}\|\right)
$$

and $\mathbf{r}$ is the deviatoric stress-ratio

\section{Equation 64 - Deviatoric Stress Ratio}

$$
\mathbf{r}=\frac{1}{p} \mathbf{S}
$$

The parameter $k_{d}$ determines a dilatancy surface, whereas the parameter $\xi$ scales the evolution of dilational plastic flow. The back stress evolves according to an Armstrong-Frederick type hardening law

Equation 65 - DPKH Armstrong-Frederick Hardening Law, see Equation 2.6 of (Pisano, et. al., 2012)

$$
\dot{\boldsymbol{\alpha}}=\frac{2}{3} h_{a}\left(\boldsymbol{\varepsilon}^{p}\right)^{d e v}-c_{r} \boldsymbol{\alpha} \sqrt{\frac{2}{3}\left\|\left(\boldsymbol{\varepsilon}^{p}\right)^{d e v}\right\|^{2}}
$$

See [25] for more details. Even with the added sophistication of the DPKH model, it turns out to be difficult to perfectly match the damping and dissipation curves, even if the model is augmented with viscous dissipation. The best fit is obtained using "calibration method 3", as illustrated in Figure 63. Note the relatively poor fit to both the modulus degradation and damping relationships. 

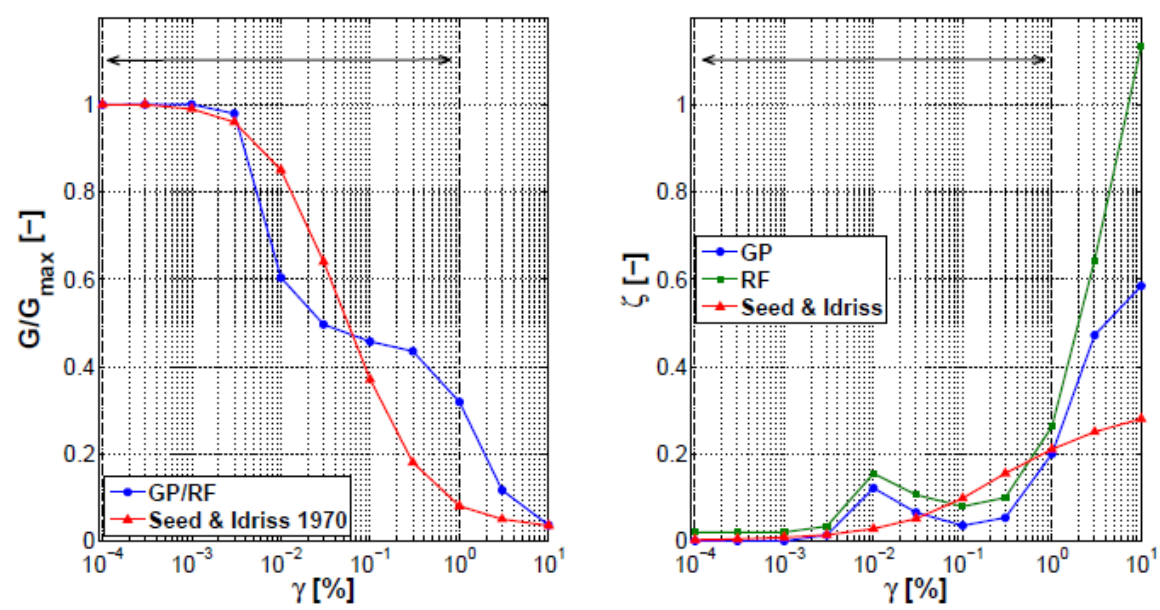

Figure 63 - Calibration 3 of the DPKH model versus typical shear modulus degradation and damping curves [Seed and Idris] - see Figure 2.19 of (Pisano, et. al., 2012)

Given these difficulties, the authors proceed to develop their own model, based upon the material strength being limited by two surfaces simultaneously. The first bounding surface is of familiar DruckerPrager form

Equation 66 - Pisano-Jeremic Yield Function - see Equation 3.6 of (Pisano, et. al, 2012)

$$
f=\frac{3}{2}\|\mathbf{s}-p \boldsymbol{\alpha}\|^{2}-k^{2} p^{2}
$$

The second bounding surface serves to limit the total shear stress, again as a function of the pressure

Equation 67 - Pisano-Jeremic Bounding Surface - see Eqution 3.8 of (Pisano, et. al., 2012)

$$
f_{B}=\frac{3}{2}\|\mathbf{S}\|^{2}-M^{2} p^{2}
$$

A non-associative flow rule essentially equivalent to Equation 62 is used, however the dilatancy parameter is calculated differently, via

$$
D=\xi\left(\sqrt{\frac{2}{3}} k_{d} \mathbf{n}^{d e v}-\boldsymbol{\alpha}\right) \bullet \mathbf{n}^{d e v}
$$

Where $\mathbf{n}^{d e v}$ is the normal to the yield function, projected to the deviatoric stress space. A deviatoric plastic flow rule is assumed.

The most interesting development is the fact that the equations hold together at the limit when $k$ in Equation 66 goes to zero - in which case the purely elastic region vanishes. In this condition, the stress response is governed entirely by the hardening laws, limited by the bounding surface of Equation 67. 
With a vanishing elastic region, plasticity (and hence dissipation), can be driven even at vanishingly small elastic strains - in contrast to the DPKH or Von Mises results.

The developments are complicated, and [25] should be consulted for more details. But the result is that experimental shear modulus degradation and damping curves can be matched much more accurately, even without additional viscous dissipation. In fact, under constant pressure shearing an explicit expression has been obtained for the shear modulus degradation curve:

Equation 68 - Pisano-Jeremic Shear Modulus Degradation Curve - see Equation 3.33 of (Pisano, et. al., 2012)

$$
1=\frac{G}{G_{\max }}\left[1+\frac{6 G_{\max }}{h p_{0} \gamma_{\max }} \int_{0}^{\gamma_{\max }}\left(\frac{\gamma}{\tau_{\lim } / G-2 \gamma+\gamma_{\max }}\right)^{m} d \gamma\right]
$$

Figure 64 documents the application of Equation 68 to actual shear modulus degradation and damping curves. It is noted that excellent agreement with the degradation curves is obtained, but that damping is not as well matched, tending to overshoot at higher strains (and hence the addition of viscous damping does not help). But notice that damping has been obtained even down to zero strain.

The authors recommend, at this point, that some manual tuning be used to improve the fit, along with a bit of viscous damping. This may degrade the agreement with modulus degradation, but improve the damping characteristics. The result of manual tuning to the same data used in Figure 64 is presented in Figure 65. Note that accuracy of the fit to the modulus data has been sacrificed, in order to bring the damping more in line with the experimental curves.

In contrast to the simple Ramberg-Osgood model used in the vast majority of the results reported in this document, the Pisano-Jeremic model explicitly accounts for the effect of confining pressure - which can vary significantly during a seismic event as a function of rocking or "raft uplift", as demonstrated by Figure 66. As such, under simple shear conditions the model can be calibrated to be operated differently depending on whether the material is isochoric, dilative, or contractive under simple shear conditions - see Figure 66. 

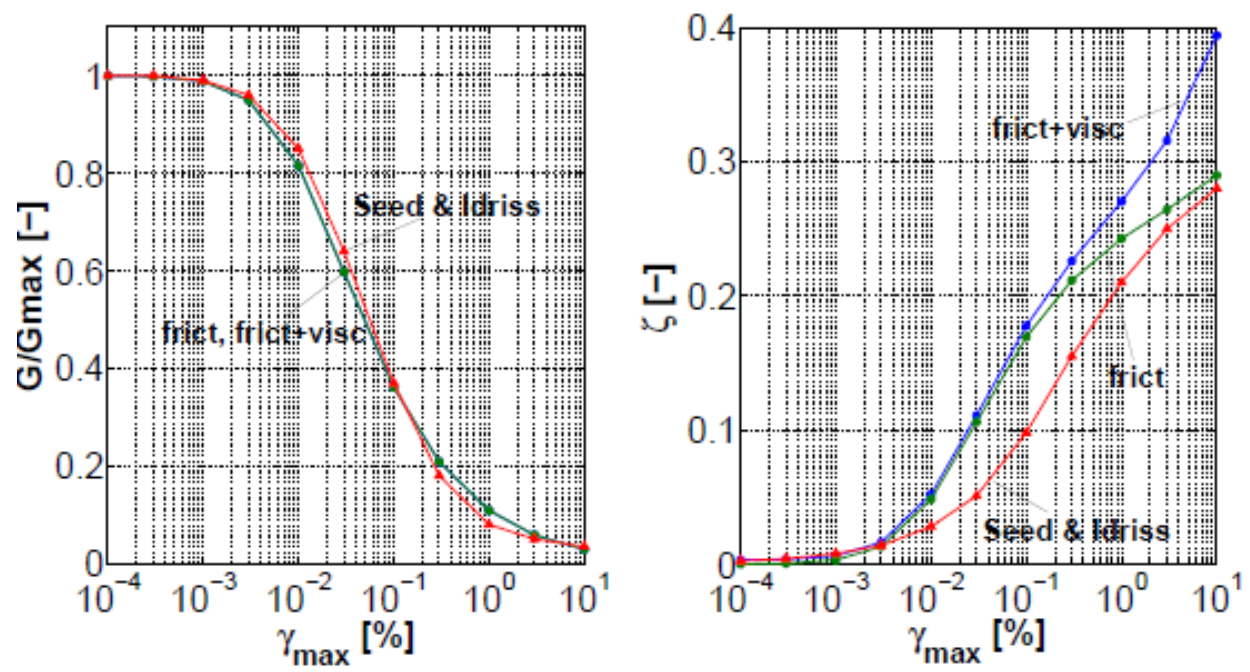

Figure 64 - Shear Modulus Degradation and Damping Curves - Comparison to Experimental Data, using Equation 3.33 of (Pisano, et. all, 2012) - see Figure 3.6 of the aforementioned work
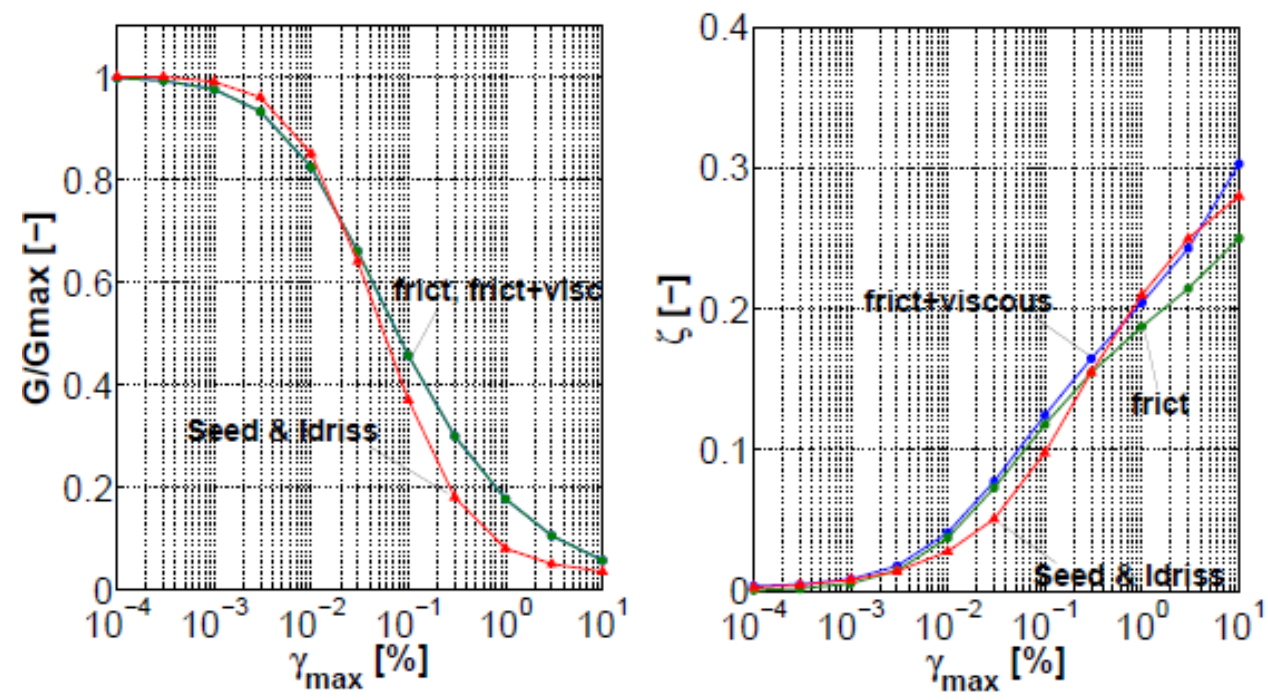

Figure 65 - Calibration of the Pisano-Jeremic model with some manual tuning - see Figure 3.7 of (Pisano, et. al., 2012) 

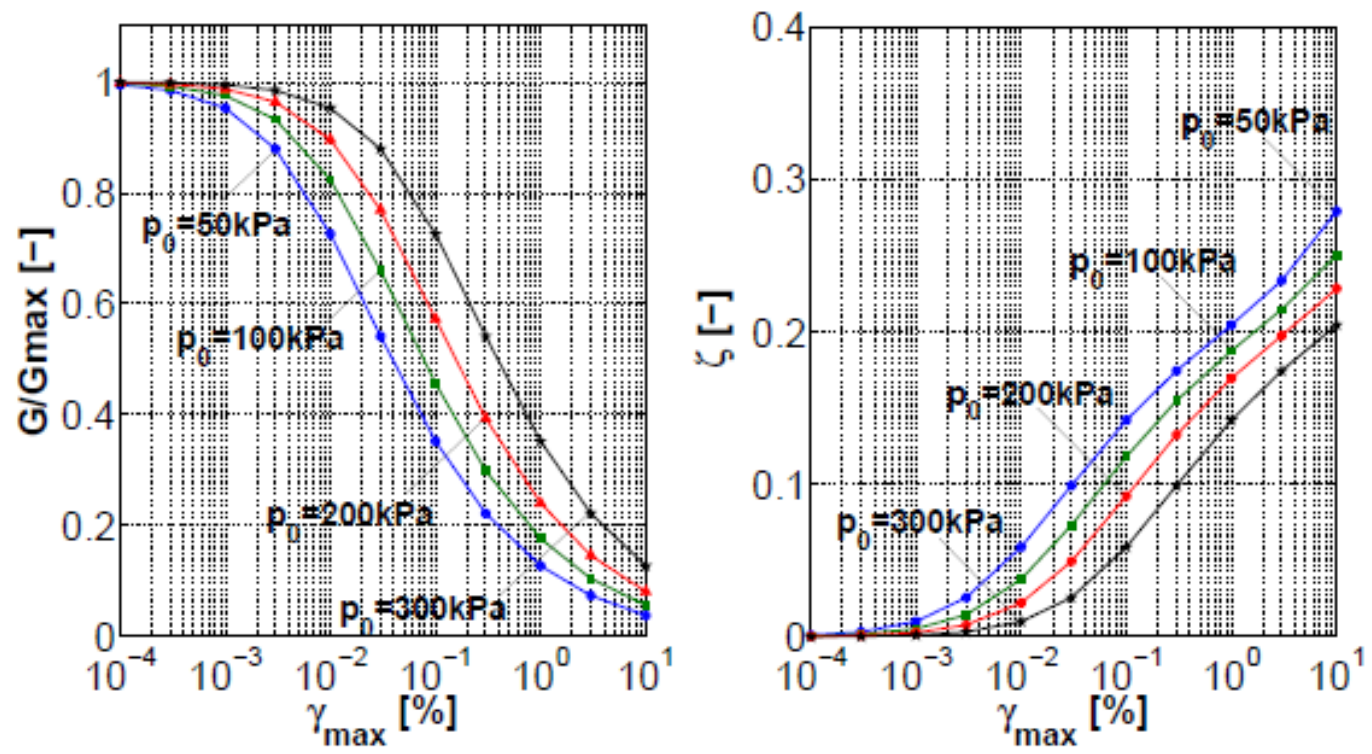

Figure 66 - Effect of varying confining pressure on the Pisano-Jeremic model, see Figure 3.8 of (Pisano, et. al., 2012)
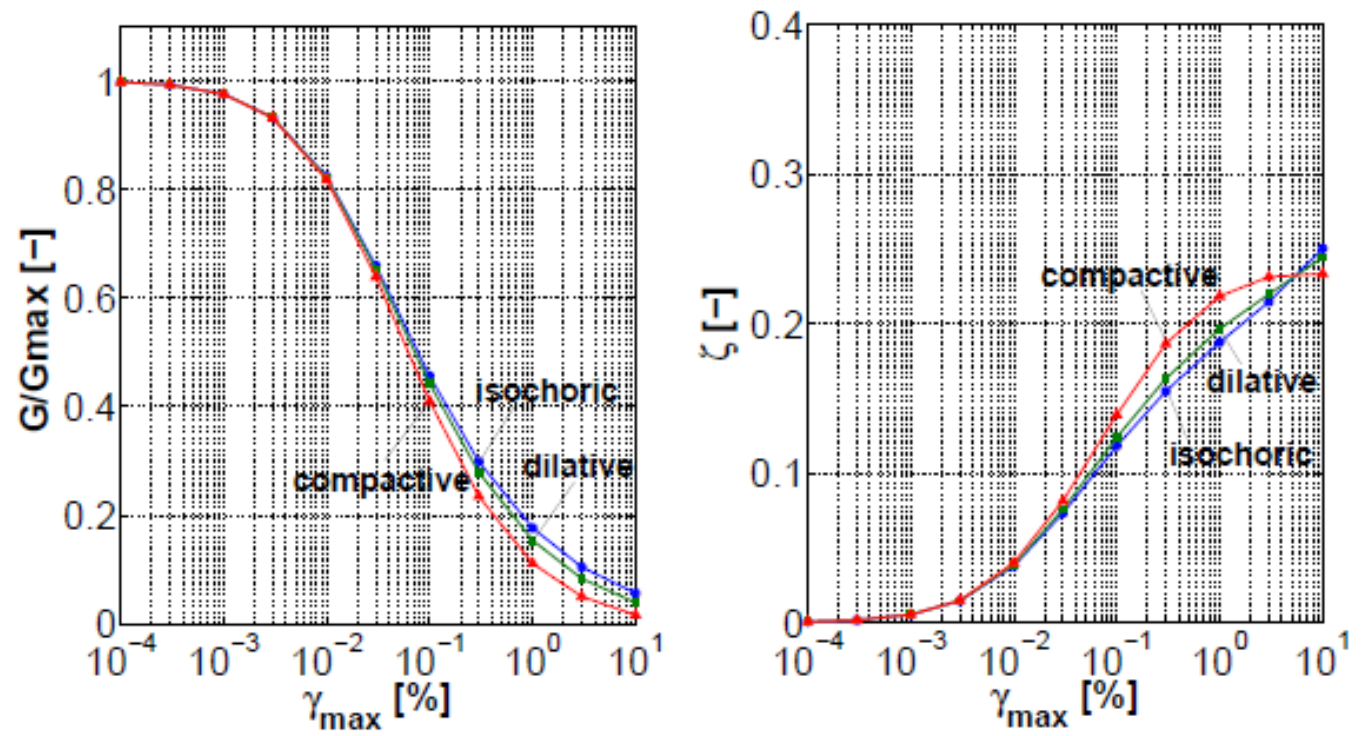

Figure 67 - Effective of different dilatancy characteristics on the response of the soil - see Figure 3.12 of (Pisano, et. al., 2012)

The model has been developed with a small number of parameters and captures the essential characteristics of experimental shear modulus degradation and damping data. An explicit expression has been developed which can be used to match experimental data. With additional tuning and the addition of some viscous dissipation the fit can be made sharper. In compensation for the added complexity of the model (relative to the Ramberg-Osgood model) the model accurately captures the inherent 3D response of the soil, especially in terms of pressure dependence. 
Some possible extensions of the model include

- Shear strength sensitivity to tension/compression

- Brittle behavior at high densities

- Fabric effects

- Visco-plasticity

\subsection{Time-domain SSI analysis of notional SMR Reactor and Comparison to SASSI results}

In order to provide a comprehensive test of the nonlinear Bielak Method and the Ramberg-Osgood model, a comparison to a SASSI analysis of a notional embedded reactor was performed. The development of the reactor model and the soil profile was documented in sections 3.1 .1 and 3.1.3. The "improved nonlinear Bielak Method" with impedance matching, represented by Equation 50, was used. The inner soil island of the Bielak model was a $100 \mathrm{~m} \times 100 \mathrm{~m}$ (50m on each side) right hexahedron, extending uniformly from the bedrock to the surface. The intermediate Bielak layer was $10 \mathrm{~m}$ thick. The development of the Ramberg-Osgood coefficients was documented in 3.4.2. For comparison purposes, as number of points within the model were chosen, see Figure 68, at which the response spectra of the SASSI analysis results were compared to DIABLO output. All DIABLO used an implicit Newmark time stepping method ("average acceleration") and a Broyden-type quasi-Newton nonlinear solution scheme.

The simulation was performed on LLNL's open side high-capcity linux cluster "RZMerl", using 16 nodes and 128 processors. A single analysis time block of 12 hours (12 hours wall-time) was sufficient to analyze 26.3 minutes of "shake time", corresponding to 42,071 steps at a constant step size of $6.25 \mathrm{E}-04$ seconds, about 2.2 minutes of "shake" per wall-time-hour. The solution required on average 3 nonlinear equilibrium iterations per step. 


\begin{tabular}{|c|c|c|c|c|c|}
\hline Location Description & $\begin{array}{c}\text { DIABLO Model } \\
\text { Node Number }\end{array}$ & $\begin{array}{c}\text { SASSI Model } \\
\text { Node Number }\end{array}$ & $\begin{array}{c}\text { X Coordinate } \\
(\mathrm{m})\end{array}$ & $\begin{array}{c}Y \text { Coordinate } \\
(\mathrm{m})\end{array}$ & $\begin{array}{c}\text { Z Coordinate } \\
(\mathrm{m})\end{array}$ \\
\hline Base Mat Center & 1319 & 1942 & 0.00 & 0.00 & -34.50 \\
\hline 1st Floor Center & 435 & 14522 & 3.13 & 0.00 & -22.56 \\
\hline 2nd Floor Center & 675 & 27948 & 3.13 & 0.00 & -9.14 \\
\hline Reactor Top Cover & 27 & 32546 & 0.00 & 0.00 & -4.50 \\
\hline 3rd Floor Center & 3436 & 38557 & 0.00 & 0.00 & 6.50 \\
\hline Roof Centr & 4140 & 39962 & 0.00 & 0.00 & 13.50 \\
\hline & & & & & \\
\hline Base Mat Edge $\mathrm{X}=14$ & 1384 & 2949 & 14.00 & 0.00 & -34.50 \\
\hline 1st Floor Edge $\mathrm{X}=14$ & 2055 & 15095 & 14.00 & 0.00 & -22.56 \\
\hline 2nd Floor Edge $\mathrm{X}=14$ & 2676 & 28514 & 14.00 & 0.00 & -9.14 \\
\hline 3rd Floor Edge $\mathrm{X}=14$ & 3321 & 38876 & 14.00 & 0.00 & 6.50 \\
\hline Roof Edge $\mathrm{X}=14$ & 3962 & 40333 & 14.00 & 0.00 & 13.50 \\
\hline & & & & & \\
\hline Base Mat Edge $\mathrm{Y}=14$ & 1700 & 1887 & 0.91 & 14.00 & -34.50 \\
\hline 1st Floor Edge $\mathrm{Y}=14$ & 2363 & 14364 & -0.18 & 14.00 & -22.56 \\
\hline 2nd Floor Edge $\mathrm{Y}=14$ & 2993 & 27782 & 0.18 & 14.00 & -9.14 \\
\hline 3rd Floor Edge $\mathrm{Y}=14$ & 3493 & 38569 & 0.00 & 14.00 & 6.50 \\
\hline Roof Edge $\mathrm{Y}=14$ & 4145 & 39976 & 0.00 & 14.00 & 13.50 \\
\hline
\end{tabular}

Figure 68 - Location of Comparison Points between SASSI and DIABLO

The problem was driven by displacement boundary conditions at the base of the solution domain. Lysmer boundary conditions were imposed along the sides of the solution domain. The imposed displacements were entirely X-directed, representing vertically propagating shear waves as in the 1D soil column runs of Section 3.4.3. At a future date, comparisons of vertical input motions, and combined vertical/shear motions, will be investigated.

Simultaneously, SASSI simulations of the same problem were performed by J. Nie of BNL and coworkers/subcontractors and are documented in [107]. The work used the Modified Subtraction Method (MSM). A cutoff frequency of $29.3 \mathrm{~Hz}$ (frequency number 1200 in SASSI) was used. The numbers of actually calculated frequencies were determined (iteratively), based on assessment of the transfer functions, and they are listed below for each case:

- $0.2 \mathrm{~g}: 52$

- $0.5 \mathrm{~g}: 55$

- $0.9 g: 47$

A comparison of peak lateral acceleration values is provided in Figure 69, and peak vertical acceleration values in Figure 70. Inspection of Figure 69, for the horizontal accelerations shows:

1. DIABLO and SASSI are in reasonable agreement for all three levels of earthquake inputs, and for all five floor elevation levels. Generally (but not universally), SASSI predicts slightly higher maximum accelerations in comparison with DIABLO. This is likely due to the nonlinear nature of DIABLO's material response - in the case of large motions the Ramberg-Osgood material model will strain-soften and increase the damping in comparison with the fixed values (derived from 
the SHAKE predictions) used by SASSI. This effect is particularly in evidence for the response at the Basemat.

2. The horizontal acceleration response at a given location increases with the acceleration level of the driving earthquake.

3. Horizontal acceleration responses are generally (but not universally) higher at higher locations in the building.

Inspection of Figure 70, for the vertical accelerations shows:

1. DIABLO and SASSI are in reasonable agreement at the structure edge for all three earthquake motions, and for all five floor elevation levels.. At most elevation levels, SASSI predicts slightly lower vertical accelerations than DIABLO.

2. At the structure centerline, DIABLO and SASSI are in reasonable agreement at the first and second floor elevations for all three earthquake motions, but SASSI predicts very low vertical accelerations (compared to DIABLO) at the other elevation levels. This is probably due to the nonlinear character of DIABLO - at small response levels the Ramberg-Osgood model will be stiffer and have less damping than the fixed values (derived from the SHAKE predictions) used by SASSI.

3. The vertical acceleration response at a given location increases with the acceleration level of the driving earthquake.

4. There is not a major variation in vertical acceleration response higher locations in the building.

5. All the vertical accelerations are small compared to the horizontal accelerations;. Note that, in the figures, the graphical scale for the vertical accelerations is magnified by a factor of 3 compared to the horizontal accelerations.

6. The vertical accelerations at the structure edge are much higher than at the structure centerline, indicating that both models are capturing the rocking motion of the structure within the soil. 

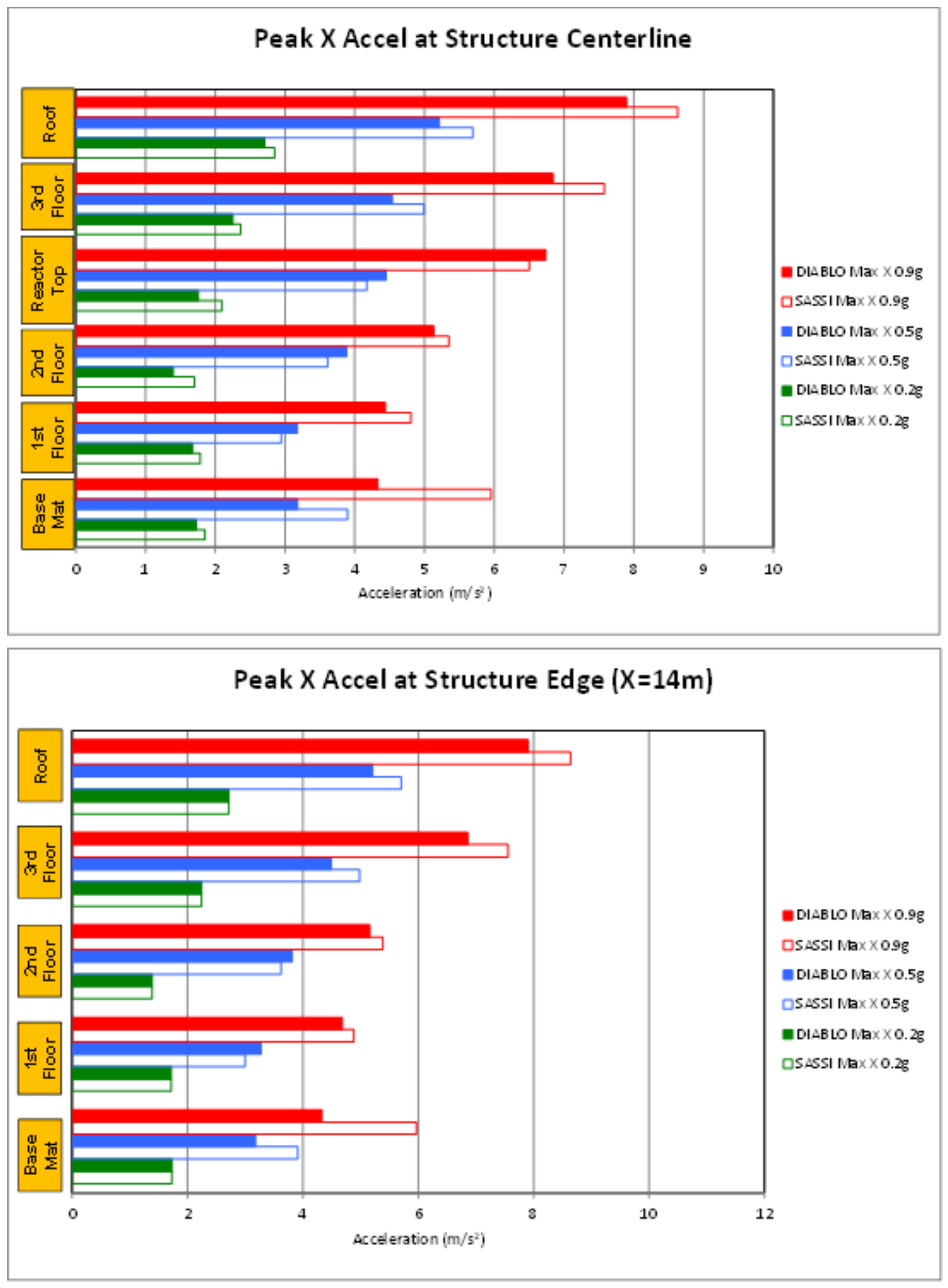

Figure 69 - Peak horizontal accelerations at the structure centerline and edge, for three earthquake motions, calculated by DIABLO and SASSI 

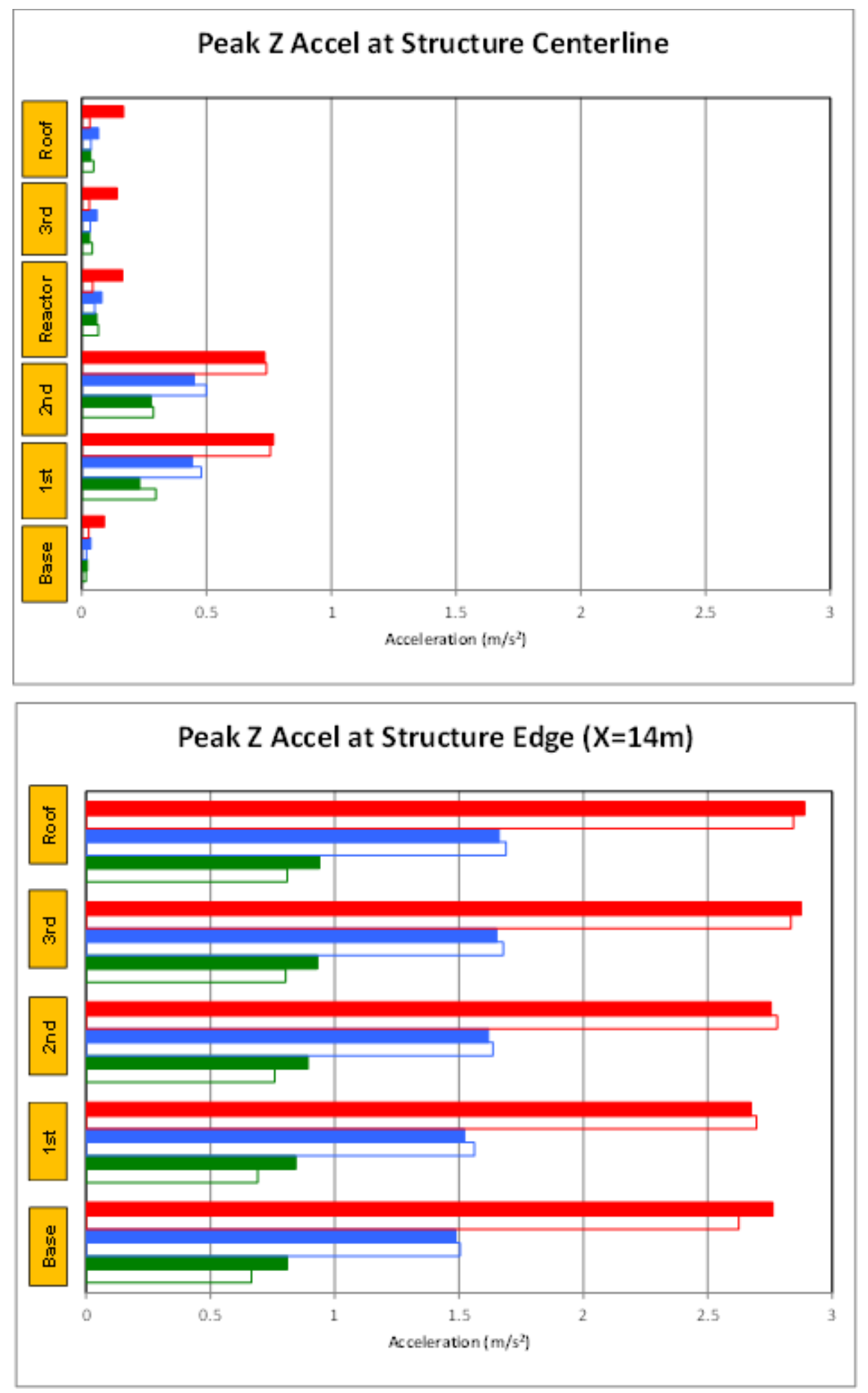

Figure 70 - Peak vertical accelerations at the structure centerline and edge, for three earthquake motions, calculated by DIABLO and SASSI 
A sample of response spectra at the various locations of interest are reproduced as Figure 71, Figure 72, and Figure 73. The complete data sets are provided in the Appendix B.
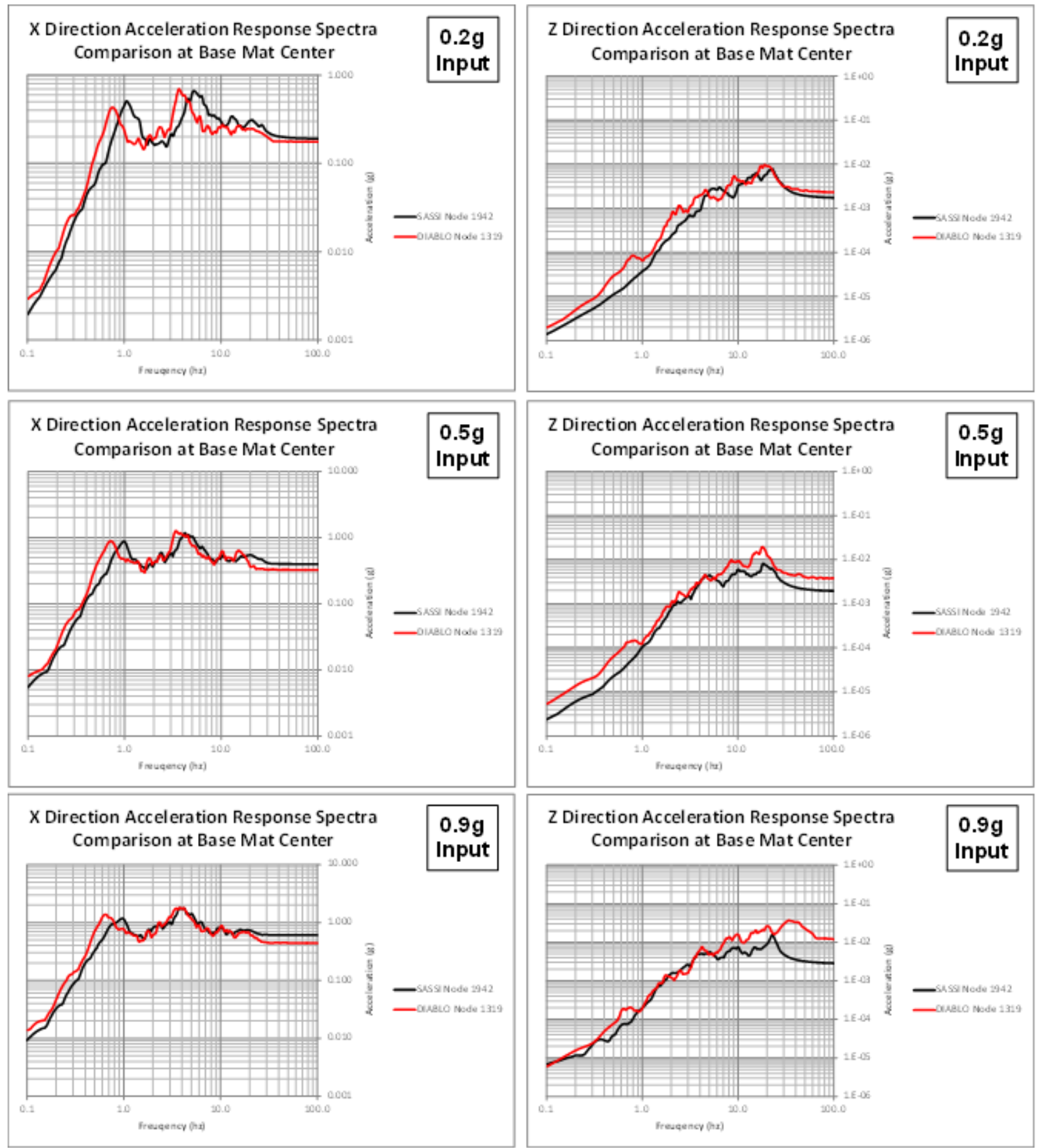

Figure 71 - Comparison of SASSI (black) and DIABLO (red) at the center of the basemat

In Figure 71, the $x$ (horizontal) and $z$ (vertical) responses are shown at the center of the basemat. The response spectra match well except for the Z-direction component, which is of relatively small magnitude, where the SASSI results are lower than the DIABLO results. For the $\mathrm{X}$ component, the peak 
frequencies are shifted relative to SASSI - most probably a function of the non-linear character of the DIABLO simulation.
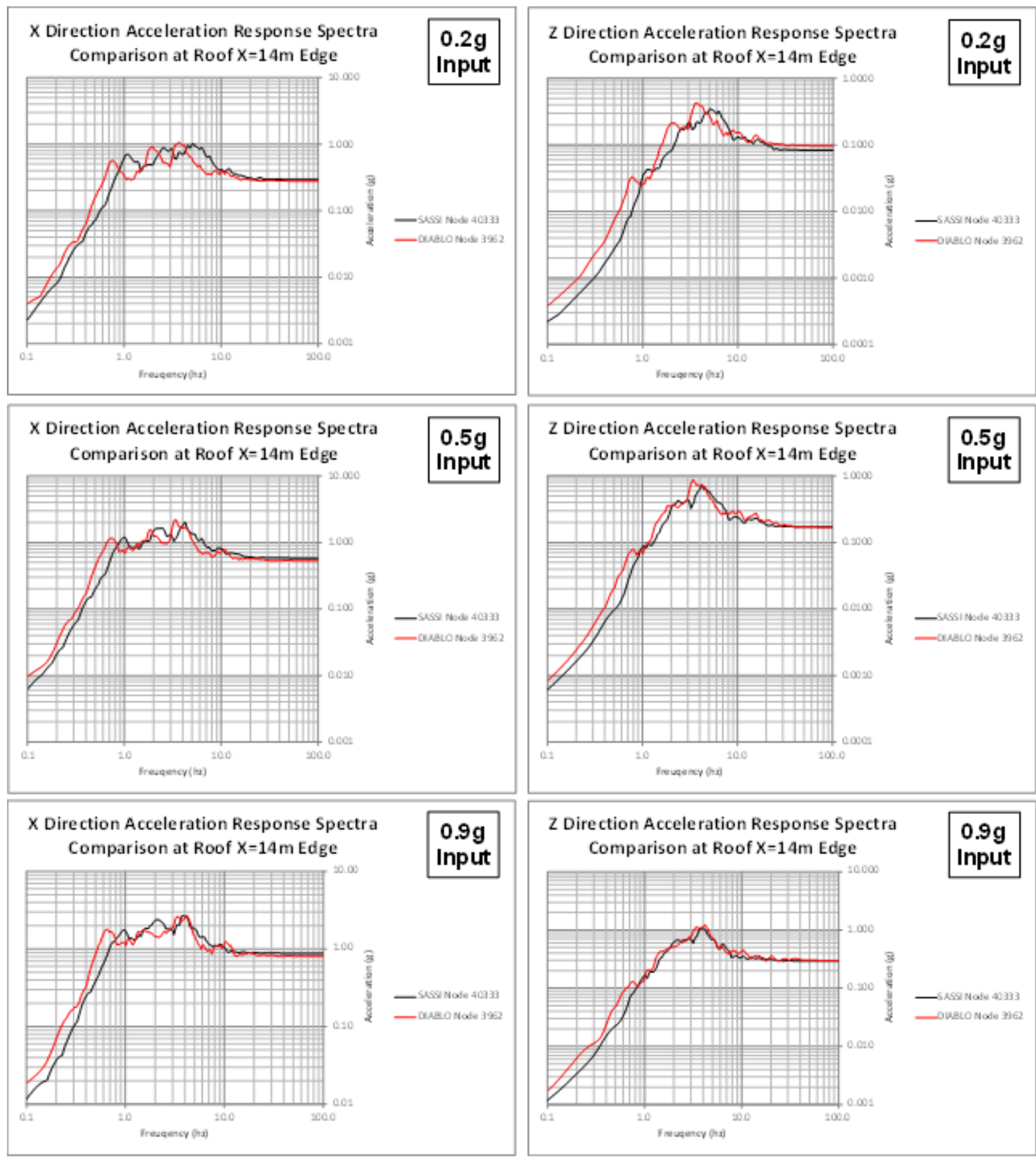

Figure 72 - Comparison of SASSI (black) and DIABLO (red) at the Roof Edge 
In Figure 72, representing the response at the roof edge, both the horizontal and vertical responses match well. The response is large for all cases, due to the significant rocking motions experienced by the reactor, which are felt at this location since it is off-center. Though the general shape of the response matches well, the peak frequencies again are shifted. Frequency shifts are not uncommon when comparing the response of a non-linear model and an equivalent linear approximation.
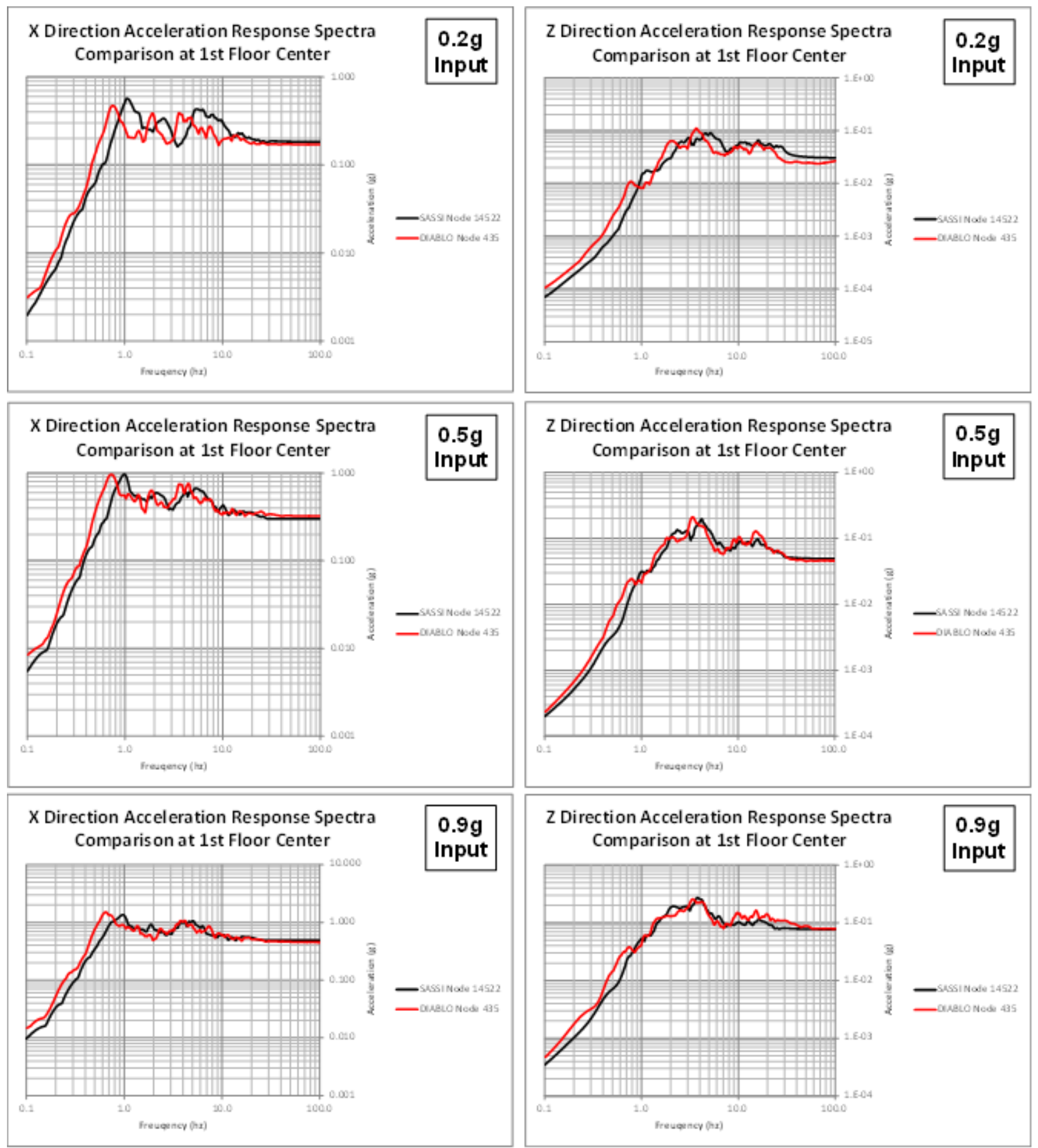

Figure 73 - Comparison of SASSI (black) and DIABLO (red) for the $1^{\text {st }}$ Floor Center 
In Figure 73, the response at the structure edge, on the first floor, generally matches well except at some high frequencies. Again, the peak frequencies are shifted, especially for the $0.2 \mathrm{~g}$ and $0.5 \mathrm{~g}$ cases.

A comparison of the response spectra indicates that the two models produce similar results, except at lower response levels (see Figure 71, Z Direction). The peak frequencies are also sometimes shifted between the two codes. The differences at lower response levels, as well as the peak frequency shifts, can be attributed to the non-linear capabilities of DIABLO in comparison to SASSI. SASSI's equivalent linear approach imposes lower modulus and higher damping everywhere (all frequencies and strains). This leads to under-predicted response in regions of relatively low magnitude, since for this situation DIABLO will be both stiffer and have less damping; hence, soil/structure system resonances will both be shifted in frequency and be less damped.

Nonlinear soil models naturally form a hierarchy, where models of progressively greater complexity can account for more and more of the response characteristics of actual soils. The Ramberg-Osgood model is a particularly simple form of nonlinear soil model, chosen for the ease by which it is matched to EPRI data for the Savannah River Site soil column. The Ramberg-Osgood model was developed primarily to model 1-D response to shear waves and was extended to 3-D response in a very simple manner. As such it is a member of the family of "tier 0" models that are simple generalizations of 1-D models [23], [24]. It does not incorporate numerous features of advanced soil models, including pressure dependence and cyclic degradation. Thus, it is to be expected that the Ramberg-Osgood results are largely similar to the SASSI results. In fact, this is a significant milestone, as it demonstrates a method whereby, through proper choice of material model and time-domain substructuring approach (the modified Bielak method), results comparable to SASSI can be generated in the time-domain using modern computational tools. This means that a path forward has been generated whereby the impact of progressively more complex (and physically realistic) models can be evaluated, with traceability to SASSI analysis.

\subsubsection{Effect of Using Equation 50 instead of Equation 41}

The system was also analyzed using the nonlinear Bielak method but without using the modified strain developed to ensure that the stresses vary smoothly across the intermediate layer - that is, using Equation 41 instead of the "impedance matched" method of Equation 50. Without modification, the strain within the layer is not a linearly scaled version of the strain within the central region - hence (because the soil is nonlinear) the impedances are mismatched at the layer boundaries. This impedance mismatch causes spurious wave reflection, polluting the solution. The peak acceleration values obtained without implementing the modified is plotted with reference to SASSI in Figure 74. Comparing these results versus the "impedance matched" results of Figure 69 and Figure 70, it is evident that the non-impedance matched solution has quite a bit larger peak accelerations, especially for the Zcomponent and for the reactor top cover. A comparison of DIABLO spectral response with and without impedance matching is provided in Figure 75. The difference in spectral response is quite remarkable, especially at high frequencies - the result of spurious wave reflection generated by the non-impedancematched solution. 

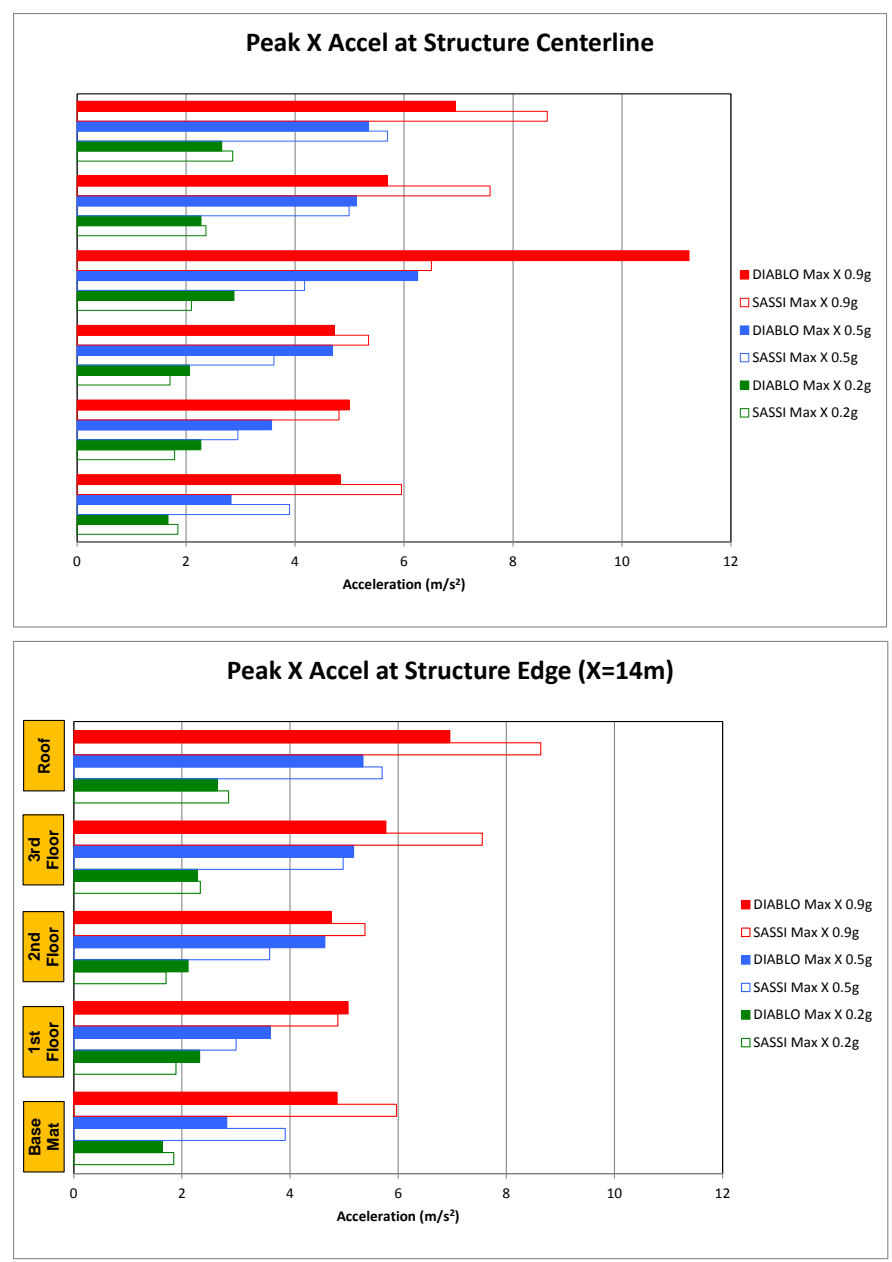

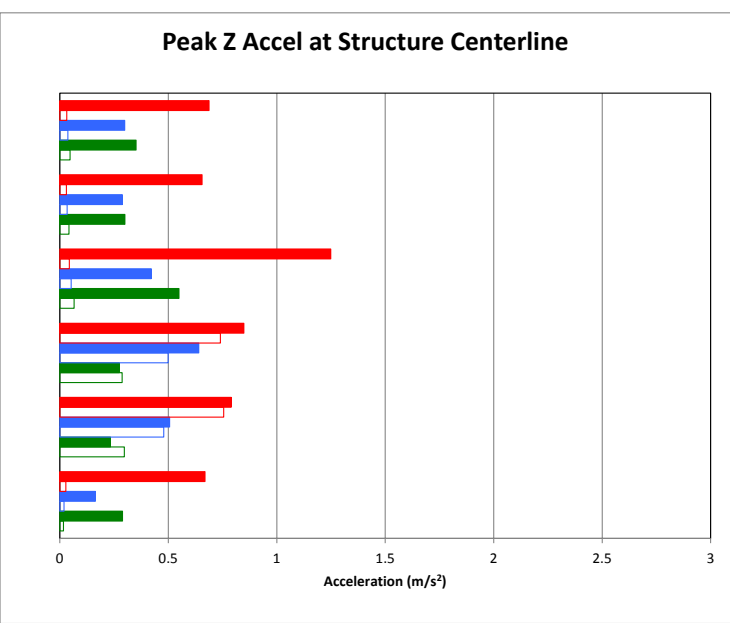

Peak Z Accel at Structure Edge $(X=14 \mathrm{~m})$

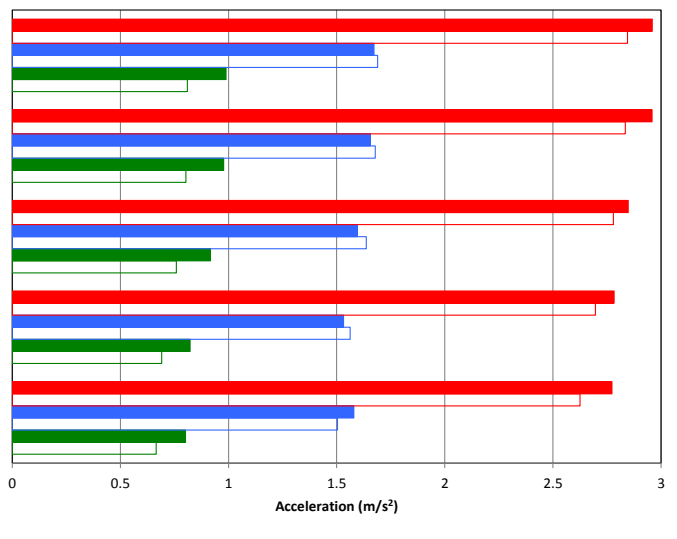

Figure 74 - Comparison of Peak Acceleration values, SASSI (open) versus DIABLO WITHOUT Impedance matching (filled) 

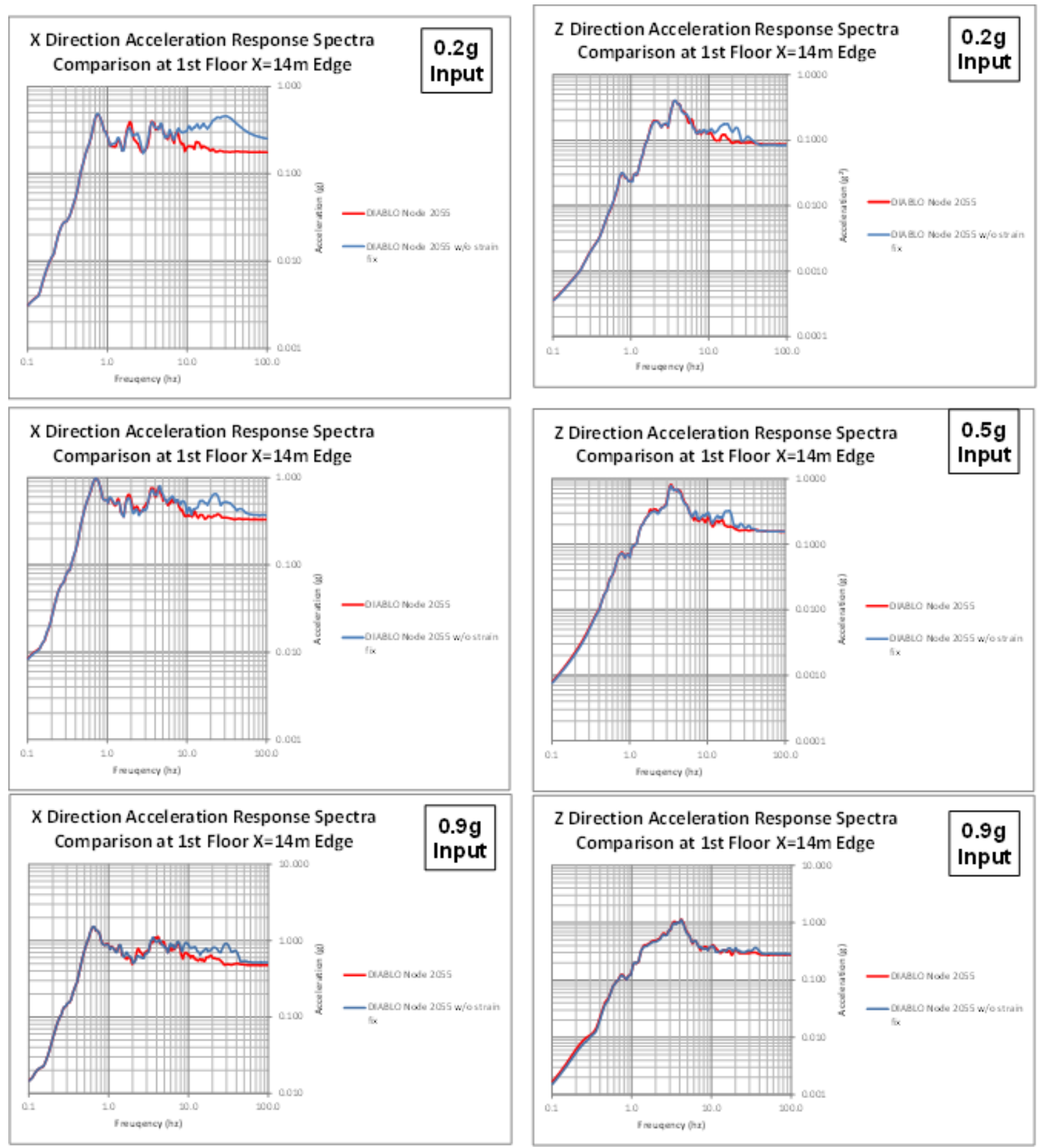

Figure 75 - Comparison of DIABLO with impedance matching (red) and without (blue), 1st Floor edge X=14 


\subsection{Conclusions and Recommendations}

Proper modeling of soils requires accounting for nonlinear behavior even down to very small strains. Frequency-domain analysis, as represented by SASSI, cannot properly account for the numerous sources of non-linearity present in soils. The "equivalent linear method", often used in concert with SASSI, can only approximately account for some non-linearities, and can underpredict the structural response if not used very carefully by experts. Time-domain analysis, capable of fully accounting for nonlinear response, is the appropriate method to adequately account for the full range of soil-structure interaction.

LLNL developed a modified Bielak method for boundary conditions, which facilitates efficient modeling of soil-structure interaction in the time domain. This method is capable of driving seismic inputs for time domain solutions with arbitrary wave incidence, and for materials which are nonlinear even at zero strain levels. The method is relatively insensitive to choice of absorbing boundary conditions, and can be utilized irrespective of the presence or absence of rigid (bedrock) material at the boundary. The method is also compatible with contact interfaces, gravity initialization, and nonlinear structural elements. A 3-D input motion was developed for testing with the method at a later date.

A nonlinear Ramberg-Osgood model was used to create a nonlinear hysteretic time-domain material model with the ability to capture shear modulus degradation and damping data as per the EPRI [4] protocol. Good agreement was found between time domain solutions using the model and 1-D soil column simulations in the frequency domain performed by CARES [19]. The method was then applied to a simplified reactor model [107] and compared to the same model analyzed by the typical SASSI frequency domain approach. Reasonable agreement was found between the new time domain method using the Ramberg-Osgood material model and SASSI for seismic motions ranging from near zero to 0.9g. In contrast with the current SASSI approach, the method takes advantage of modern parallel processing computational techniques. A single analysis requires less than a day of computer wall time on $\sim 100$ processors. In addition, a means to use fully 3-D seismic inputs derived from geologic-scale wave propagation codes has been developed.

The results of the benchmarking calculations in this study verify the adequacy, in the context of simple soil models, of SASSI-calculated seismic response at large amplitudes, but clearly show the limitations of the SASSI methodology at high frequencies and/or low strains..

A hierarchy of more sophisticated soil models with traceability to EPRI test data was created [25]. The most sophisticated model, the Pisano-Jeremic model based upon a Drucker-Prager yield condition, was shown to provide good match to EPRI data and at the same time capture realistic pressure-dependent soil behavior.

The time domain model is capable of calculating soil and structural response for more complex systems and with more of the physical features, including:

- More sophisticated soil models as detailed in [25].

- Nonlinear Structural Elements including concrete damage models

- Contact/Release conditions along the structure/soil interface

- Pore pressure evolution 


\subsection{Recommendations for Future Work}

The work has developed and demonstrated the value of a time-domain method, and shown how it can be used to effectively duplicate the results of SASSI analysis without the limitations inherent in the SASSI approach. A few short term goals lead directly from the work performed so far:

- Evaluate the result of combined horizontal and vertical inputs and compare to SASSI. This would allow for the evaluation, at the same time, of typical combinatorial approaches for SASSI results, as SASSI is typically run separately for the two cases and the results combined using various "addition rules"

- Evaluate the effect of the developed 3D input motions as compared with an equivalent vertically-propagating shear wave.

- Evaluate the effect of a fitted Pisano-Jeremic material model in comparison to the DIABLO/Ramberg-Osgood and SASSI results.

- Evaluate the influence of simple contact/release conditions at the soil/structure interface.

- Apply the results of these improvements to SMR demonstration plant designs and to existing power plants whose seismic risk level has increased due to the recent NRC/EPRI Study [28].

Longer-term goals include:

- Use of SANISAND and SANICLAY (Dafalias-Manzari models), as well as other sophisticated soil models as they are developed.

- Incorporation of Porous Diffusion Model (additional global field - pore pressure)

- Incorporation of nonlinear structural elements

- Concrete Damage models

- Structural Joints - Hysteretic Damping for Structures in the Time Domain

- Composite Concrete Structures

- Nonlinear structural elements - specifically isolators

- Incorporation of pore-pressure-aware friction laws for soil/structure contact 


\subsection{Bibliography}

[1] J. Lysmer, M. Tabatabaie-Raissi, F. Tajirian, S. Vahdani and F. Ostadan, "SASSI - A System for Analysis of Soil-Structure Interaction," Geotechnical Engineering Division, 1981.

[2] J. A. Blink and R. J. Budnitz, "NEAMS, SHARP Integrated Performance and Safety Codes - Seismic LLNL - Gap Workshop Report," Livermore, CA, 2012.

[3] F. Ostadan, SASSI 2000 User's Manual, Version 3, 2007.

[4] EPRI, "Guidelines for determining design basis ground motions, Final Report," 1993.

[5] I. M. Idriss, J. I. Sun, P. Schnabel, J. Lysmer and H. B. Seed, "Users Manual for SHAKE 91, A Computer Program for Conductiving Equivalent Linear Seismic Response Analyses of Horizontally Layered Soil Deposits," National Institute of Standards, Gainsville, Maryland, 1992.

[6] J. Zhang, R. D. Andrus and C. H. Juang, "Normalized Shear Modulus and Material Damping Ratio Relationships," Journal of Geotechnical and Geoenvironmental Engineering, vol. 131, no. 4, pp. 453-464, April 2005.

[7] N. Matasovic and M. Vucetic, "Cyclic Characterization of Liquifiable Sands," Journal of Geotechnical Engineering, vol. 119, no. 11, pp. 1805-1822, November 1993.

[8] D. Assimaki and E. Kausel, "An equivalent linear algorithm with frequency- and pressuredependent moduli and damping for the seismological analysis of deep sites," Soil Dynamics and Earthquake Engineering, vol. 22, pp. 950-965, 2002.

[9] L. F. Bonilla, R. J. Archuleta and D. Lavallee, "Hysteretic and Dilatant Behavior of Cohesionless Soils and Their Effects on Nonlinear Site Response: Field Data Observations and Modeling," Bulletin of the Seismological Society of America, vol. 95, no. 6, p. 2373-2395, December 2005.

[10] F. Z. Zerfa and B. Loret, "Coupled dynamic elastic-plastic analysis of earth structures," Soil Dynamics and Earthquake Engineering, vol. 23, p. 435-454, 2003.

[11] J. P. Wolf and C. Song, "Some cornerstones of dynamic soil-structure interaction," Engineering Structures, vol. 24, pp. 13-28, 2002.

[12] S. H. Crandall, "The Role of Damping in Vibration Theory," Journal of Sound and Vibration, vol. 11, no. 1, pp. 3-18, 1970.

[13] United States Department of Energy, US. Department of Energy Report on Technical and Software Quality Assurance Issues Involving the System for Analysis of Soil-Structure Interaction ((Response 
to Defense Nuclear Facilities Board Letter dated April 8, 2011), 2011.

[14] V. A. Drosos, N. Gerolymos and G. Gazetas, "Constitutive model for soil amplification of ground shaking: Parametercalibration, comparisons, validation," Soil Dynamics and Earthquake Engineerg, vol. 42, pp. 255-274, 2012.

[15] K. Soga and C. O'Sullian, "Modeling of Geomaterials Behavior," Soils and Foundations, vol. 50, no. 6, pp. 861-875, December 2010.

[16] J. Bielak and P. Christiano, "On the Effective Seismic Input for Non-Linear Soil-Structure Interaction Systems," Earthquake Engineering and Structural Dynamics, vol. 12, pp. 107-119, 1984.

[17] J. Bielak, K. Loukakis, Y. Hisada and C. Yoshimura, "Domain Reduction Method for ThreeDimensional Earthquake Modeling in Localized Regions, Part I: Theory," Bulletin of the Seismological Society of America, vol. 93, no. No. 2, p. 817-824, April 2003.

[18] J. Xu and C. J. Costantino, "CARES (Computer Analysis for Rapid Evaluation of Structures) Version 1.0 - Vol. 1 - Seismic Module Theoretical Manual, Vol. 2 - Seismic Module User's Manual, Vol. 3 Seismic Module Sample Problems," Nuclear Regulatory Commission, 1990.

[19] C. J. Costantino, C. Miller and J. Xu, "CARES: Computer Analysis for Rapid Evaluation of Structures Version 1.2," Constantino, Miller and Associates, Monsey, N.Y., 1995.

[20] W. Ramberg and W. R. Osgood, "Description of Stress-strain curves by three parameters," National Advisory Committee for Aeronautics, USA (NACA), 1943.

[21] T.-S. Ueng and J.-C. Chen, "Computational Procedures for Determining Parameters in RambergOsgood Elastoplastic $\mathrm{M}<$ odel Based on Modulus and Damping versus Strain," Lawrence Livermore National Security, Livermore, CA, 1992.

[22] G. Waas, R. Riggs and H. Werkle, "Displacment Solutions for Dynamic Loads in TransverselyIsotropic Stratified Media," Earthquake Engineering and Structural Dynamics, vol. 13, pp. 173-193, 1985.

[23] D.-Y. Chiang, "The generalized Masing models for deteriorating hysteresis and cyclic plasticity," Applied Mathematical Modeling, vol. 23, pp. 847-863, 1999.

[24] D. J. Segalman and D. J. Starr, "Inversion of Masing models via continuous Iwan systems," International Journal of Non-Linear Mechanics, vol. 43, pp. 74-80, 2008.

[25] F. Pisano and B. Jeremic, "Soil Material Modeling for Department of Energy Small Modular Reactor Modeling and Simulation Project," Davis, 2012. 
[26] L. Hutchings, "DOE-Nuclear Engineering Project, Ground Motion Input for Soil-Structure Interaction," 2012.

[27] M. P. S. d' Avila, L. Lentil and J.-F. Semblat, "Modelling strong seismic ground motion: threedimensional loading path versus wavefield polarization," Geophysical Journal International, vol. 190, p. 1607-1624, 2012.

[28] G. Stirewalt, L. Salamone, S. McDuffie, K. Coppersmith, Fugro William Lettis \& Associates, C. Fuller, R. Hartleb, W. Lettis, S. Lindvall, R. McGuire, G. Toro, G. Slayter, R. Cumbest, A. Shumway, F. Syms, AMEC Geometrix, Inc., L. Glaser, K. Hanson, R. Youngs, S. Bozkurt, V. M. Faler, R. Perman and M. Tuttle, "Central and Eastern United States Seismic Source Characterization for Nuclear Facilities (CEUS-SSC)," Nuclear Regulatory Commission (NRC), 2012.

[29] American Society of Civil Engineers, "Seismic Analysis of Safety-Related Nuclear Structures," American Society of Civil Engineers (ASCE), 2000.

[30] T. Blacker, "CUBIT 12.1 User Documentation," Albuquerque, NM, 2010.

[31] D. E. Wyatt and M. K. Harris, "Overview of the history and geology of the Savannah River Site," Environmental Geosciences, vol. 11, no. 4, pp. 181-190, December 2004.

[32] P. B. Schnabel, J. M. Lysmer and H. B. Seed, SHAKE, A Computer Program for Earthquake Response Analysis of Horizontally Layered Sites, Berkeley, CA: Earthquake Engineering Research Center, University of California at Berkeley, 1972.

[33] O. C. Zienkiewicz and R. L. Taylor, The Finite Element Method, Its Basis and Fundamentals, Sixth Edition, vol. I, Woburn, MA: Butterworth-Heinemann, 2005.

[34] N. M. Newmark, "A method of computation for structural dynamics," Journal of Engineering Mechanics, ASCE, vol. 85, no. EM3, pp. 67-94, 1958.

[35] C. Phillips, A. Kottke, Y. M. A. Hashash and E. M. Rathje, "Significance of ground motion time step in one dimensional site response analysis," Soil Dynamics and Earthquake Engineering, vol. 43, pp. 202-217, 2012.

[36] J. Lysmer, "Analytical Procedures in Soil Dynamics," University of California, Berkeley, 1978.

[37] J. Lysmer, T. Udaka, H. B. Seed and R. Hwang, "LUSH - A computer program for complex response analysis of soil-structure systems," Earthquake Engineering Research Center, Berkeley, 1974.

[38] M. Vucetic and R. Dobry, "Effect of Soil Plasticity on Cyclic Response," Journal of Geotechnical Engineering-ASCE, vol. 117, no. 1, pp. 89-107, January 1991. 
[39] I. Ishibashi and X. Zhang, "Unified Dynamic Shear Moduli and Damping Ratios of Sand and Clay," Soils and Foundations, vol. 33, no. 1, pp. 182-191, March 1993.

[40] C. Phillips and M. A. H. Youssef, "Damping formulations for nonlinear 1D site response analyses," Soil Dynamics and Earthquake Engineering, vol. 29, pp. 1143-1158, 2009.

[41] D. K. Reyes, A. Rodriguez-Marek and A. Lizcano, "A hypoplastic model for site response analysis," Soil Dynamics and Earthquake Engineering, vol. 29, pp. 173-184, 2009.

[42] C. Gellis and L. F. Bonilla, "2-D P-SV numerical study of soil-source interaction in a non-linear basin," Geophysical Journal International, vol. 191, p. 1374-1390, 2012.

[43] T. K. Caughey, "Classical Normal Modes in Damped Linear Systems," Journal of Applied Mechanics, vol. 27, pp. 269-271, 1960.

[44] J. F. Semblatt, "Rheological Interpretation of Rayleigh Damping," Journal of Sound and Vibration, vol. 206, no. 5, pp. 741-744, 1997.

[45] M. Liu and D. G. Gorman, "Formulation of Rayleigh Damping and Its Extensions," Computers and Structures, vol. 27, no. 2, pp. 277-285, 1995.

[46] G. Muscolino, A. Palmeri and F. Ricciardelli, "Time-domain response of linear hysteretic systems to deterministic and random excitations," Earthquake Engineering and Structural Dynamics, vol. 34, p. 1129-1147, 2005.

[47] J. Xu, C. Miller, C. Costantino, C. Hofmayer and H. L. Graves, "Assessment of Seismic Analysis Methodologies for Deeply Embedded Nuclear Power Plant Structures," United States Nuclear Regulatory Commission, Office of Nuclear Regulatory Research, Upton, NY, 2006.

[48] F. Ostadan, SASSI 2000 Theoretical Manual, Version 3, 2007.

[49] C. W. Bert, "Material Damping: An Introductory Review of Mathematical Models, Measures, and Experimental Techniques," Journal of Sound and Vibration, vol. 29, no. 2, pp. 129-153, 1973.

[50] E. Balmes, "Modeling Damping at the Material and Structural Level," in Proceedings of the 2006 IMAC-XXIV: Conference \& Exposition on Structural Dynamics , 2006.

[51] S. H. Crandall, "The hysteretic damping model in vibration theory," Proceedings of the Institution of Mechanical Engineers, Part C: Journal of Mechanical Engineering Science, vol. 205, pp. 23-28, 1991.

[52] B. Gross, "On Creep and Relaxation," Journal of Applied Physics, vol. 18, pp. 212-221, 1947. 
[53] D. R. Bland, The theory of Linear Viscoelasticity, Oxford: Pergamon Press, 1960.

[54] W. N. Findley, J. S. Lai and K. Onaran, Creep and Relaxation of Nonlinear Viscoelastic Materials, With an Introduction to Linear Viscoelasticity, Amsterdam: North Holland, 1976.

[55] N. Delepine, L. Lenti, G. Bonnet and J.-F. Semblat, "Nonlinear Viscoelastic Wave Propagation: An Extension of Nearly Constant Attenuation Models," Journal of Engineering Mechanics, ASCE, vol. 135, pp. 1305-1314, November 2009.

[56] G. B. Muravskii, "Linear models with nearly frequency independent complex," Earthquake Engineering and Structural Dynamics, vol. 36, pp. 13-33, 2007.

[57] F. Garcia-Moliner, "Linear Response Functions and the Phenomenological Equations of Internal Friction," Journal of Applied Physics, vol. 37, no. 1, pp. 83-89, 1966.

[58] P. R. Dahl, "Solid Friction Damping of Mechanical Vibrations," AIAA Journal, vol. 14, no. 12, pp. 1675-1682, December 1976.

[59] A. K. Padthe, B. Drincic, J. Oh, D. D. Rizos, S. D. Fassius and D. S. Bernstein, "Duhem Modeling of Friction-Induced Hysteresis - Experimental Determination of Gearbox Stiction," IEEE Control Systems Magazine, pp. 90-107, October 2008.

[60] M. Ismail, F. Ikhouane and J. Rodellar, "The Hysteresis Bouc-Wen Model, a Survey," Archive for Computer Methods in Engineering, vol. 16, pp. 161-188, 2009.

[61] G. A. Ortiz, D. A. Alvarez and D. Bedoya-Ruiz, "Identification of Bouc-Wen type models using multiobjective optimization functions," Computers and Structures, Vols. 114-115, pp. 121-132, 2013.

[62] V. Aleshin and K. Van Den Abeele, "Micro-potential model for stress-strain hysteresis of microcracked materials," Journal of the Mechanics and Physics of Solids, vol. 53, p. 795-824, 2005.

[63] A. Ivanyi, P. Ivanyi, M. M. Ivanyi and M. Ivanyi, "Hysteresis in Structural Dynamics," Physica B., vol. 407, pp. 1412-1414, 2012.

[64] G. Masing, "Self-stretching and hardening for brass," in Proceedings of the 2nd International Congress for Applied Mechanics, 1926.

[65] W. D. Iwan, "A Distributed-Element Model for Hysteresis and its Steady-State Dynamic Response," Journal of Applied Mechanics, vol. 33, no. 4, pp. 893-900, 1966.

[66] J. O. Malley, G. Dierlein, H. Krawinkler, J. R. Maffei, M. Pourzanjani, J. Wallace and J. A. Heintz, "Modeling and Acceptance Criteria for Seismic Design and Analysis of Tall Buildings," Applied Technology Council, 2010. 
[67] M. Rahnama and H. Krawinkler, "Effects of Soft Soil and Hysteresis Model on Seismic Demancs," John A. Blume Earthquake Engineering Center, Stanford, CA, 1993.

[68] R. A. Medina and H. Krawinkler, "Seismic Demands for NonDeteriorating Frame Structures and Their Dependence on Ground Motions," John A. Blume Earthquake Engineering Center, Stanford, CA, 2003.

[69] L. F. Ibarra, R. A. Medina and H. Krawinkler, "Hysteretic models that incorporate strength and stiffness deterioration," Earthquake Engineering and Structural Dynamics, vol. 34, pp. 1489-1511, 2005.

[70] I. M. Idriss, R. Dobry and R. D. Singh, "Nonlinear Response of Soft Clays during Cyclic Loading," Journal of the Geotechnical Engineering Division, ASCE, vol. 104, no. GT12, 1978.

[71] P. C. Jennings, "Periodic Response of General Yielding Structure," Journal of the Engineering Mechancis Division, ASCE, vol. 90, no. EM2, 1964.

[72] R. Pyke, "Nonlinear Soil Models for Irregular Cyclic Loadings," Journal of the Engineering Mechanics Division, ASCE, vol. 105, no. GT6, 1979.

[73] G. V. Rao and A. K. Murty, "An Alternate Form of the Ramberg-Osgood Formula for Matrix Analysis," Nuclear Engineering and Design, vol. 17, pp. 297-308, 1971.

[74] A. J. Carr, "Ruaumako: The Maori God of Volcanoes and Earthquakes, Volume I: Theory," Computer Program Library, ChistChurch, New Zealand, 2007.

[75] N. Mostaghel and R. Byrd, "Inversion of Ramberg-Osgood equation and description of hysteresis loops," International Journal of Non-Linear Mechanics, vol. 37, pp. 1319-1335, 2002.

[76] S. Personeni, M. D. Pilato, A. Palermo and S. Pampanin, "Numerical Investigations of the Seismic Reponse of Masonry Infilled Steel Frames," in Proceedings of the 14th World Conference on Earthquake Engineering, 2008.

[77] M. A. Puso, "NIKE3D - A Nonlinear, Implicit, Three-Dimensional Finite Element Code for Solid and Structural Mechanics, User's Manual," Lawrence Livermore National Security, LLC, Livermore, CA, 2012.

[78] I. D. Parsons, J. M. Solberg, R. M. Ferencz, M. A. Havstad, N. E. Hodge and A. P. Wemhoff, "DIABLO User Manual," Lawrence Livermore National Security, LLC, 2007.

[79] D. M. Wood, Soil Behavior and Critical State Soil Mechanics, Cambridge, UK: Cambridge University Press, 1990. 
[80] J. Lysmer and R. L. Kuhlemeyer, "Finite Dynamic Model for Infinite Media," Journal of Engineering Mechanics Division, ASCE, vol. 95, no. EM4, pp. 859-877, August 1969.

[81] R. Clayton and B. Enquist, "Absorbing Boundary Conditions for Acoustic and Elastic Wave Equations," Bulletin of the Seismological Society of America, vol. 67, no. 6, pp. 1529-1540, December 1977.

[82] E. Kausel and J. L. Tassoulas, "Transmitting Boundaries: A Closed-Form Comparison," Bulletin of the Seismological Socmty of America, vol. 71, no. 1, pp. 143-159, February 1981.

[83] E. Kausel, "Local Transmitting Boundaries," vol. 114, pp. 1011-1027, 1988.

[84] D. Givoli, "Non-reflecting Boundary Conditions," Journal of Computational Physics, vol. 94, pp. 129, 1991.

[85] D. Givoli, "High-order local non-reflecting boundary conditions: a review," Wave Motion, vol. 39, pp. 319-326, 2004.

[86] D. Rabinovich, D. Givoli, J. Bielak and T. Hagstrom, "A finite element scheme with a high order absorbing boundary condition for elastodynamics," Computer Methods in Applied Mechanics and Engineering, vol. 200, p. 2048-2066, 2011.

[87] D. Baffet, J. Bielak, D. Givoli, T. Hagstrom and D. Rabonovich, "Long-time stable high-order absorbing boundary conditions for elastodynamics," Computer Methods in Applied Mechanics and Engineering, Vols. 241-244, pp. 20-37, 2012.

[88] L. Kellezi, "Local transmitting boundaries for transient elastic analysis," Soil Dynamics and Earthquake Engineering, vol. 19, pp. 533-547, 2000.

[89] C. M. Mozos and J. E. Luco, "Approximate Boundaries for Finite-Element Models of Static SoilFoundation Interaction Problems," Journal of Engineering Mechanics, ASCE, pp. 648-659, October 2011.

[90] P. Bettes, "Infinite Elements," International Journal for Numerical Methods in Engineering, vol. 2, pp. 53-64, 1977.

[91] R. K. N. D. Rajapakse and P. Karasudhi, "Elastostatic Infinite Elements for Layered Half-Spaces," Journal of Engineering Mechanics, ASCE, vol. 111, pp. 1144-1158, 1985.

[92] F. Medina and R. L. Taylor, "Finite Element techniques for problems of unbounded domains," International Journal for Numerical Methods in Engineering, vol. 19, pp. 1209-1226, 1983.

[93] R. K. N. D. Rajakapse and P. Karasudhi, "An efficient elastodynamic infinite element," International 
Journal of Solids and Structures, vol. 22, no. 6, pp. 643-657, 1986.

[94] C. B. Yun, S. H. Chang, C. G. Seo and J. M. Kim, "Dynamic Infinite Elements for Soil-Structure Interaction Analysis in a Layered Soil Medium," International Journal of Structural Stability and Dynamics, vol. 7, no. 4, p. 693, December 2007.

[95] J. Su and Y. Wang, "Equivalent dynamic infinite element for soil-structure interaction," Finite Elements in Analysis and Design, vol. 63, pp. 1-17, 2013.

[96] J. P. Berenger, "A perfectly matched layer for the absorption of electromagnetic waves," Journal of Computational Physics, vol. 114, no. 2, pp. 185-200, 1994.

[97] U. Basu and A. K. Chopra, "Perfectly matched layers for time-harmonic elastodynamics of unbounded domains: theory and finite element implementation," Computer Methods in Applied Mechanics and Engineering, vol. 192, pp. 1337-1375, 2003.

[98] U. Basu, "Explicit finite element perfectly matched layer for transient three-dimensional elastic waves," International Journal for Numerical Methods in Engineering, vol. 77, pp. 151-176, 2009.

[99] S. Kucukcoban and L. F. Kallivokas, "A symmetric hybrid formulation for transient wave simulations in PML-truncated heterogeneous media," Wave Motion, vol. 50, pp. 57-79, 2013.

[100] J.-F. Semblatt, A. Gandomzadeh and L. Lenti, "A simple numerical absorbing layer method in elastodynamics," Comptes Rendus Mecanique, vol. 338, pp. 24-32, 2010.

[101] G. Festa and J.-P. Vilotte, "The Newmark scheme as velocity-stress time-staggering: an efficient PML implementation for spectral element simulations of elastodynamics," Geophysical Journal International, vol. 161, pp. 789-812, 2005.

[102] G. Festa and S. Nielsen, "PML Absorbing Boundaries," Bulletin of the Seismological Society of America, vol. 93, no. 2, April 2003.

[103] K. C. Meza-Fajardo and A. S. Papageorgiou, "A nonconvolutional, split-field, perfectly matched layer for wave propagation in isotropic and anisotropic elastic meida: stability analysis," Bulletin of the Seismological Society of America, vol. 98, pp. 1811-1836, 2008.

[104] M. Cremonini, P. Christiano and J. Bielak, "Implementation of Effective Seismic Input for SoilStructure Interaction," Earthquake Engineering and Structural Dynamics, vol. 16, pp. 615-625, 1988.

[105] C. Yoshimura, B. Jacobo, Y. Hisada and A. Fernandez, "Domain Reduction Method for ThreeDimensional Earthquake Modeling, Part II: Verification and Applications," Bulletin of the Seismological Society of America, vol. 93, no. No. 2, p. 825-840, April 2003. 
[106] S. Savadatti and M. N. Guddati, "Accurate absorbing boundary conditions for anisotropic elastic media. part 1: elliptical anisotropy," Journal of Computational Physics, vol. 231, p. 7584-7607, 2012.

[107] J. R. Nie, J. R. Braverman and M. Costantino, "Seismic Soil-Structure Interaction Analyses of a Deeply Embedded Model Reactor - SASSI Analyses," Brookhaven National Laboratory, 2012.

[108] G. Waas, "Earth vibration effects and abatement for military facilities - analysis method for footing vibrations through layered media," U.S. Army Engineer Waterways Experimental Station, Vicksburgh, 1972.

[109] E. Kausel and J. M. Roesset, "Stiffness Matrices for Layered Soils," Bulletin of the Seismological Society of America, vol. 72, no. 6, pp. 1743-1761, December 1981.

[110] A. Lion, "On the thermodynamics of fractional damping elements," Continuum Mechanics and Thermodynamics, vol. 9, pp. 83-96, 1997.

[111] J. Lysmer, T. Udaka, C.-F. Tsai and H. B. Seed, "FLUSH - A Computer Program for Approximate 3D Analysis of Soil-Structure Interaction Problems," Earthquake Engineering Research Center, Berkeley, California, 1975.

[112] J. Xu and C. J. Costantino, "NESC Note 91-54 "CARES" NUREG/CR-5588 Vol. 3 errata," Nuclear Energy Software Center, 1991.

[113] D. B. McCallen, "SPECTRA: A Subroutine for Response Spectra Generation," University of California, 1991.

[114] D. M. Ghiocel, The SASSI Flexible Volume Substructing Methodologies, Technical Note, Ghiocel Predictive Technologies, Inc., 2012.

[115] H. Takemiya, "Time-Domain Thin Layer Method For Computing Transient Response Due To Sudden/Moving Loads," Journal of Mechanics of Materials and Structures, vol. 3, no. 10, pp. 19031921, 2008. 


\section{Appendices}

Appendix A: Comparison of 1D Soil Column - CARES versus DIABLO with RambergOsgood

A.1 One-D Soil Column, CARES 0.2g versus DIABLO without added viscous damping
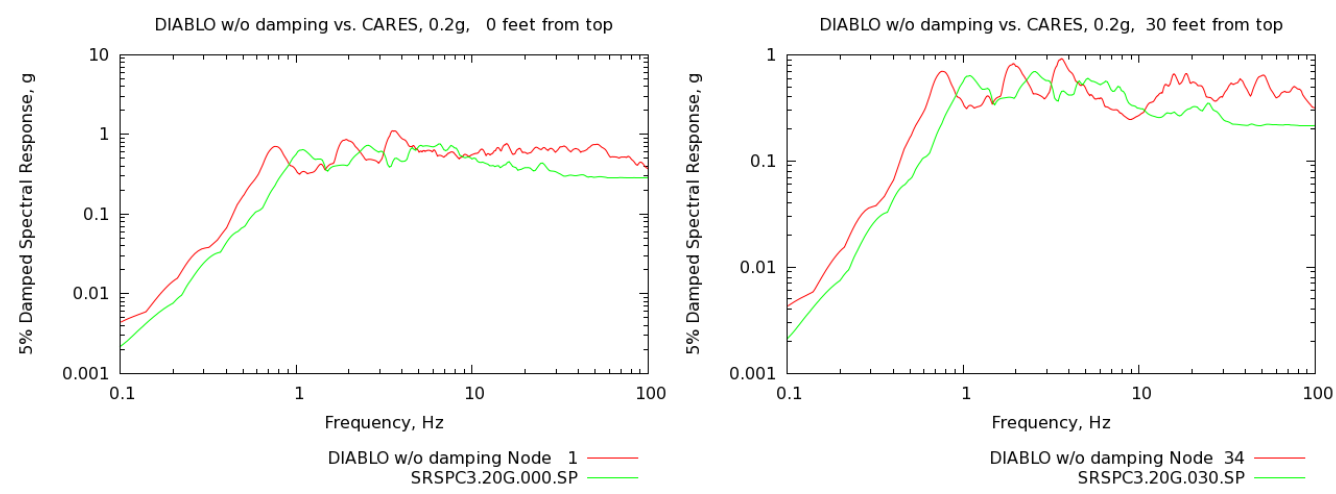

Figure 76 - 1D Soil column, 0.2g earthquake, 5\% Response Spectra: DIABLO w/o viscous damping(red) versus CARES (green), distance 0 and $30 \mathrm{ft}$ from top surface
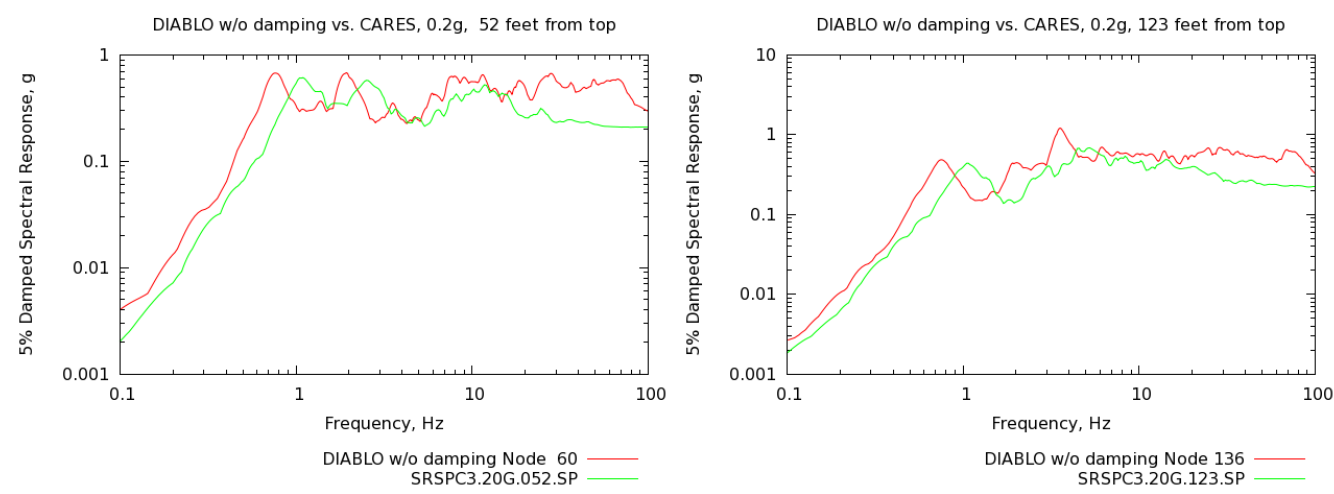

Figure 77 - 1D Soil column, 0.2g earthquake, 5\% Response Spectra: DIABLO w/o viscous damping(red) versus CARES (green), distance 52 and $123 \mathrm{ft}$ from top surface
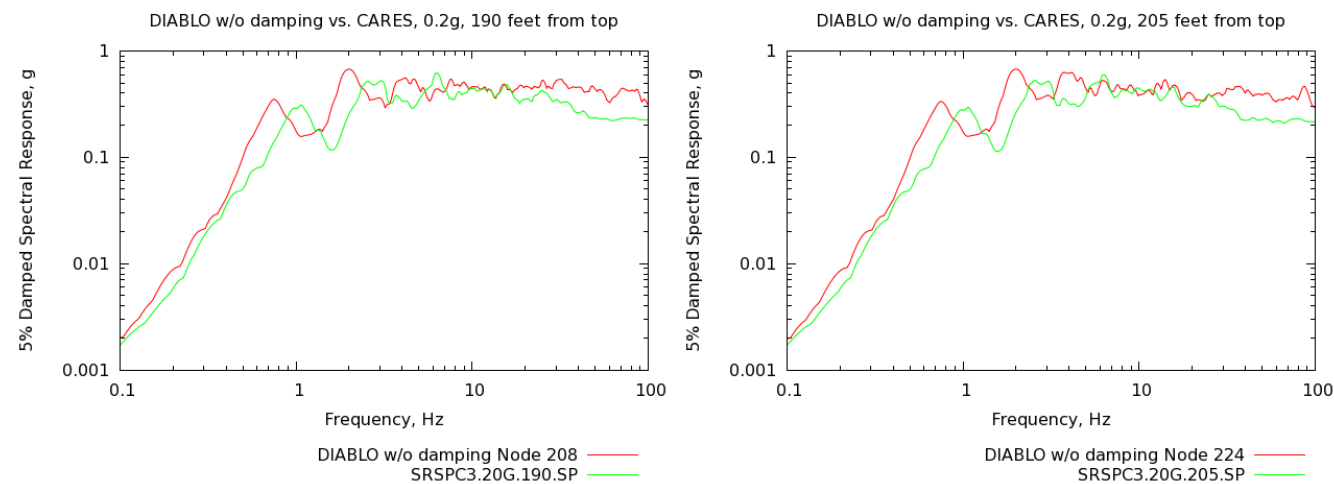

Figure 78 - 1D Soil column, $0.2 \mathrm{~g}$ earthquake, $5 \%$ Response Spectra: DIABLO w/o viscous damping(red) versus CARES (green), distance 190 and $205 \mathrm{ft}$ from top surface 

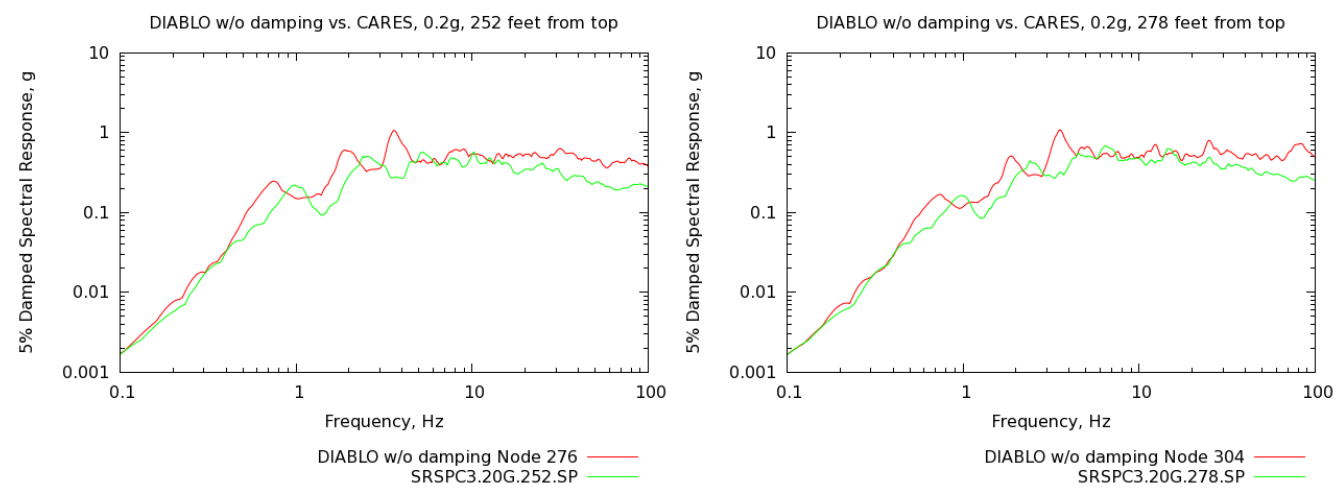

Figure 79 - 1D Soil column, $0.2 \mathrm{~g}$ earthquake, $5 \%$ Response Spectra: DIABLO w/o viscous damping(red) versus CARES (green), distance 252 and $278 \mathrm{ft}$ from top surface
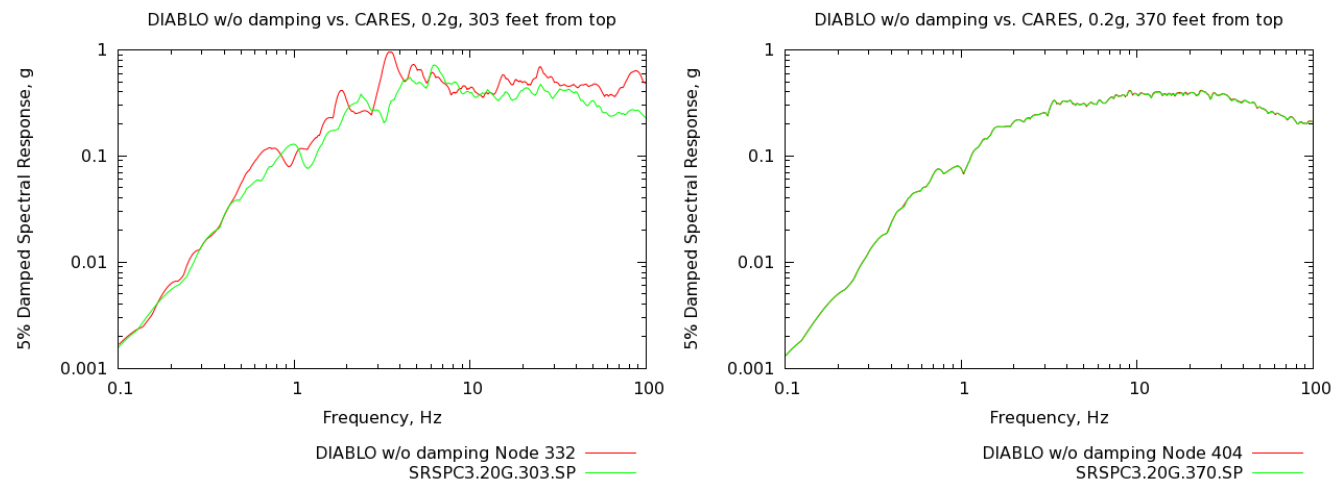

Figure 80 - 1D Soil column, $0.2 \mathrm{~g}$ earthquake, 5\% Response Spectra: DIABLO w/o viscous damping(red) versus CARES (green), distance 303 and $370 \mathrm{ft}$ from top surface 


\section{A.2 One-D Soil Column, CARES 0.2g versus DIABLO with added viscous damping}
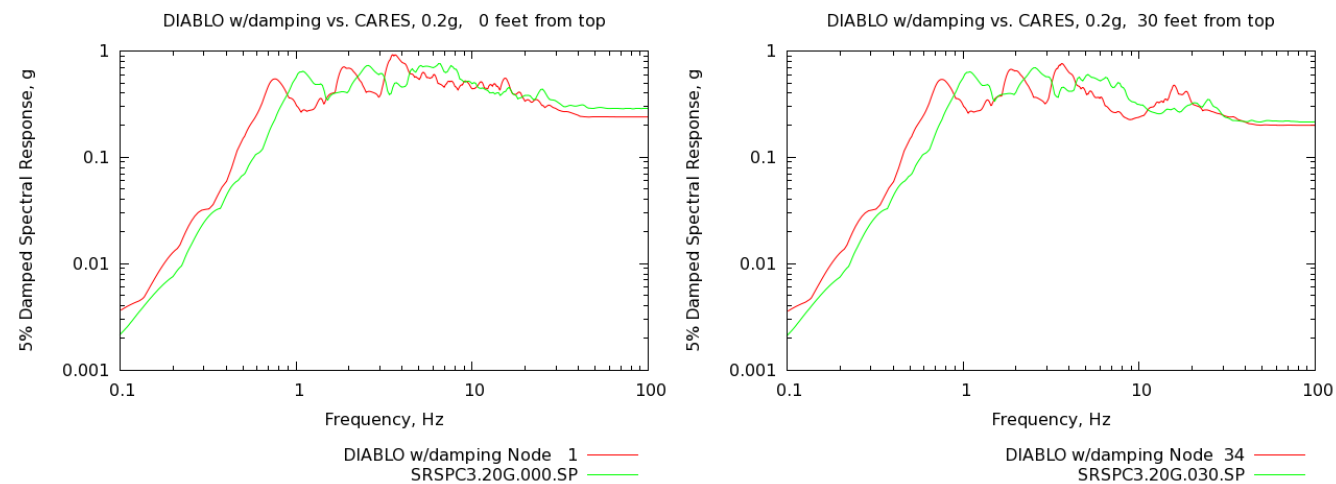

Figure 81 - 1D Soil column, $0.2 \mathrm{~g}$ earthquake, 5\% Response Spectra: DIABLO with viscous damping(red) versus CARES (green), distance 0 and $30 \mathrm{ft}$ from top surface
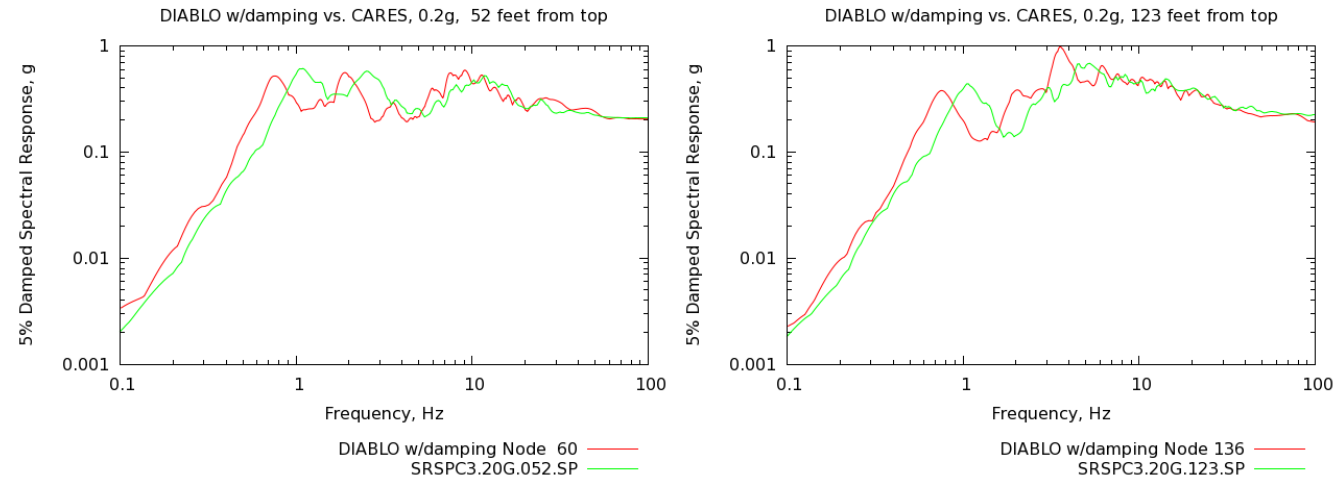

Figure 82 - 1D Soil column, 0.2g earthquake, 5\% Response Spectra: DIABLO with viscous damping(red) versus CARES (green), distance 52 and $123 \mathrm{ft}$ from top surface
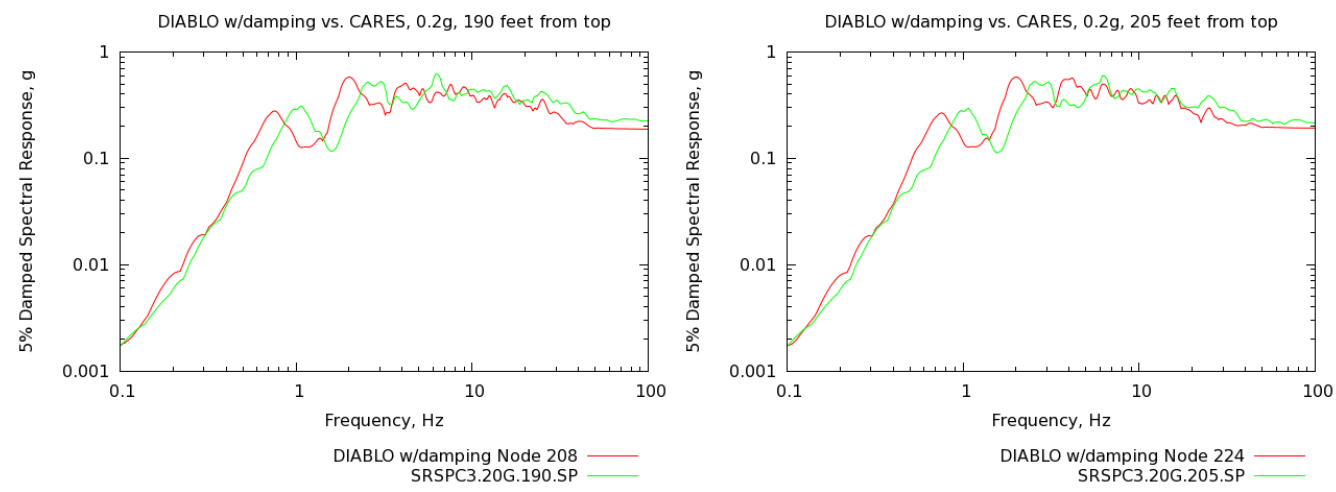

Figure 83 - 1D Soil column, $0.2 \mathrm{~g}$ earthquake, $5 \%$ Response Spectra: DIABLO with viscous damping(red) versus CARES (green), distance 190 and $205 \mathrm{ft}$ from top surface 

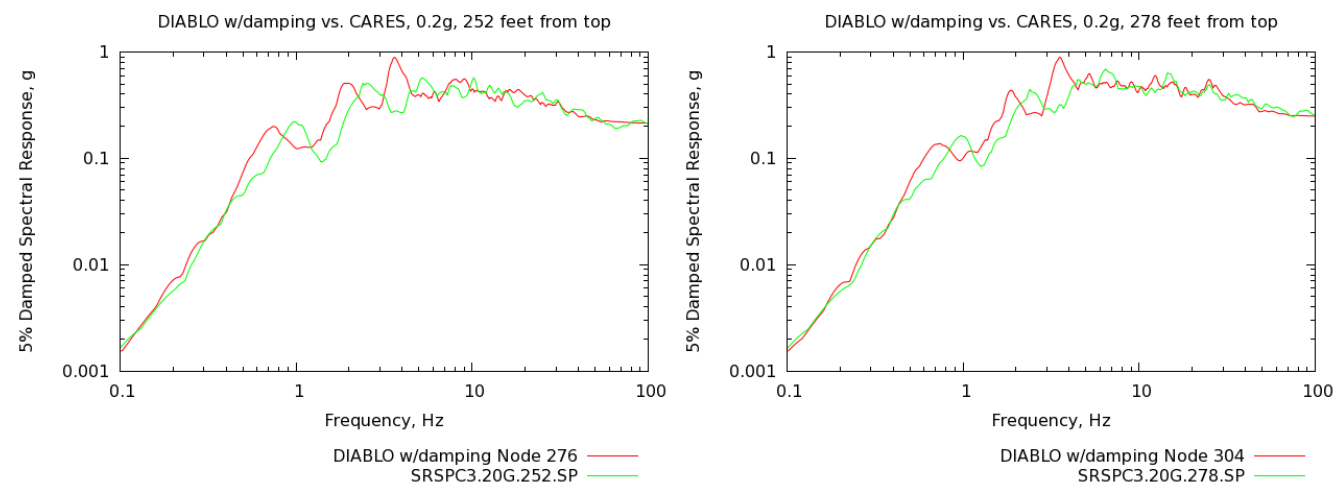

Figure 84 - 1D Soil column, 0.2g earthquake, 5\% Response Spectra: DIABLO with viscous damping(red) versus CARES (green), distance 252 and $278 \mathrm{ft}$ from top surface
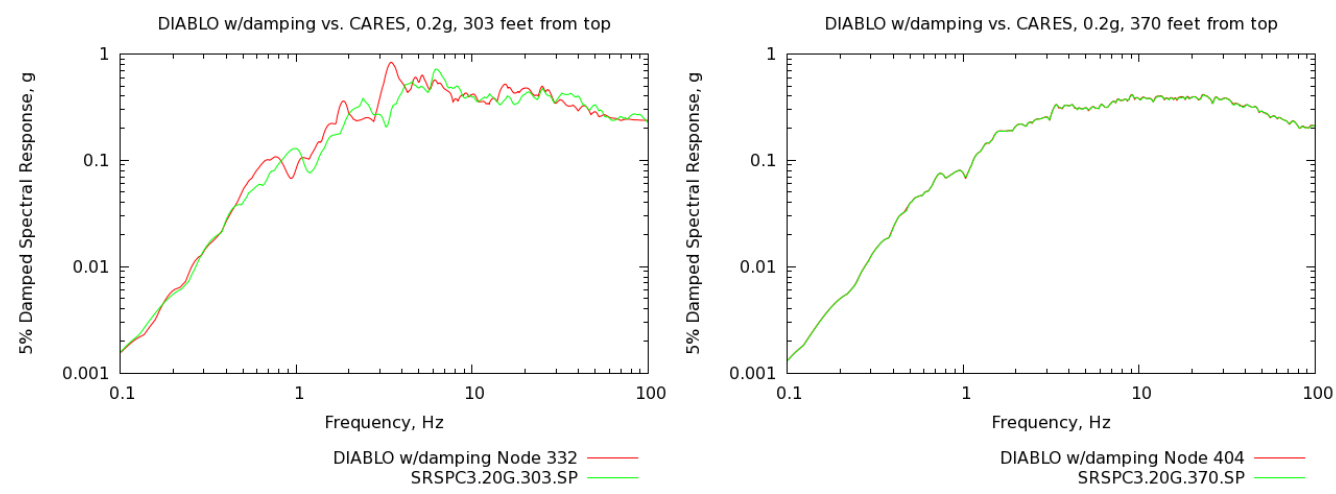

Figure 85 - 1D Soil column, 0.2g earthquake, 5\% Response Spectra: DIABLO with viscous damping(red) versus CARES (green), distance 303 and $370 \mathrm{ft}$ from top surface 


\section{A.3 One-D Soil Column, CARES 0.5g versus DIABLO with added viscous damping}
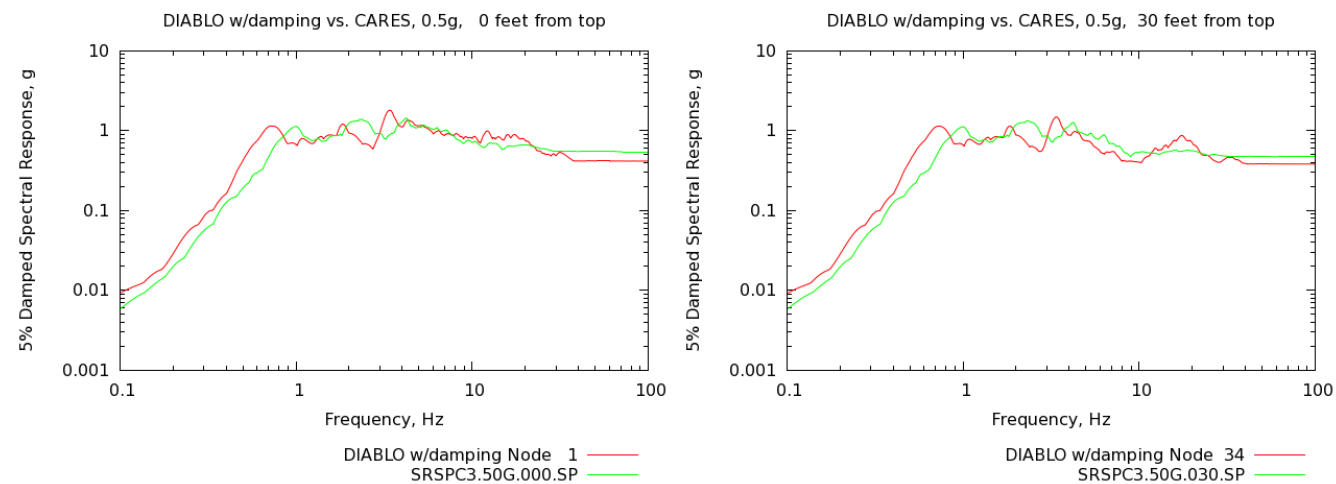

Figure 86 - 1D Soil column, 0.5g earthquake, 5\% Response Spectra: DIABLO with viscous damping(red) versus CARES (green), distance 0 and $30 \mathrm{ft}$ from top surface
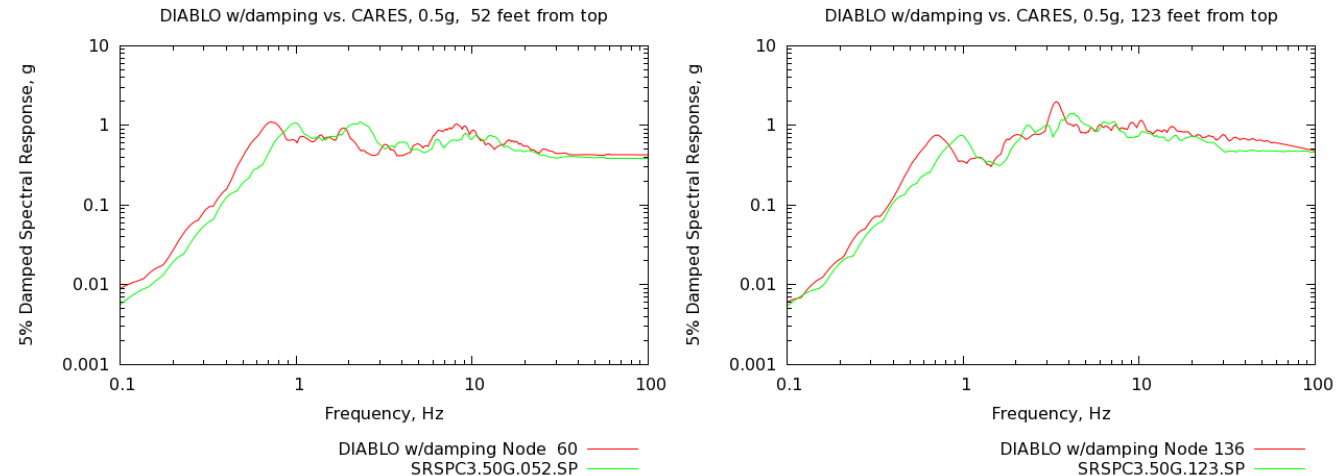

Figure 87 - 1D Soil column, 0.5g earthquake, 5\% Response Spectra: DIABLO with viscous damping(red) versus CARES (green), distance 52 and $123 \mathrm{ft}$ from top surface
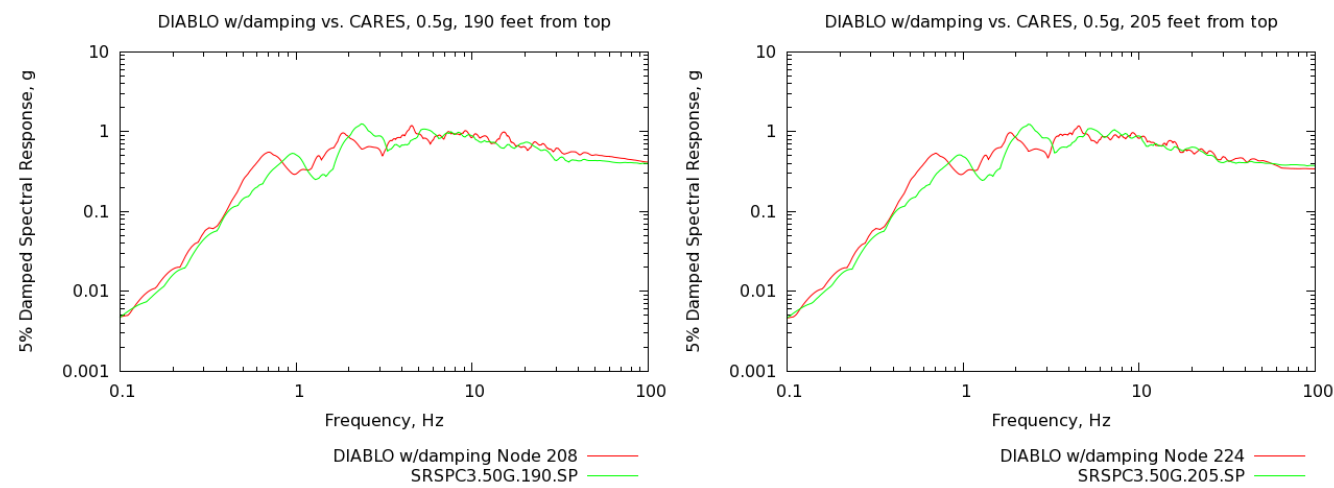

Figure 88 - 1D Soil column, $0.5 \mathrm{~g}$ earthquake, $5 \%$ Response Spectra: DIABLO with viscous damping(red) versus CARES (green), distance 190 and $205 \mathrm{ft}$ from top surface 

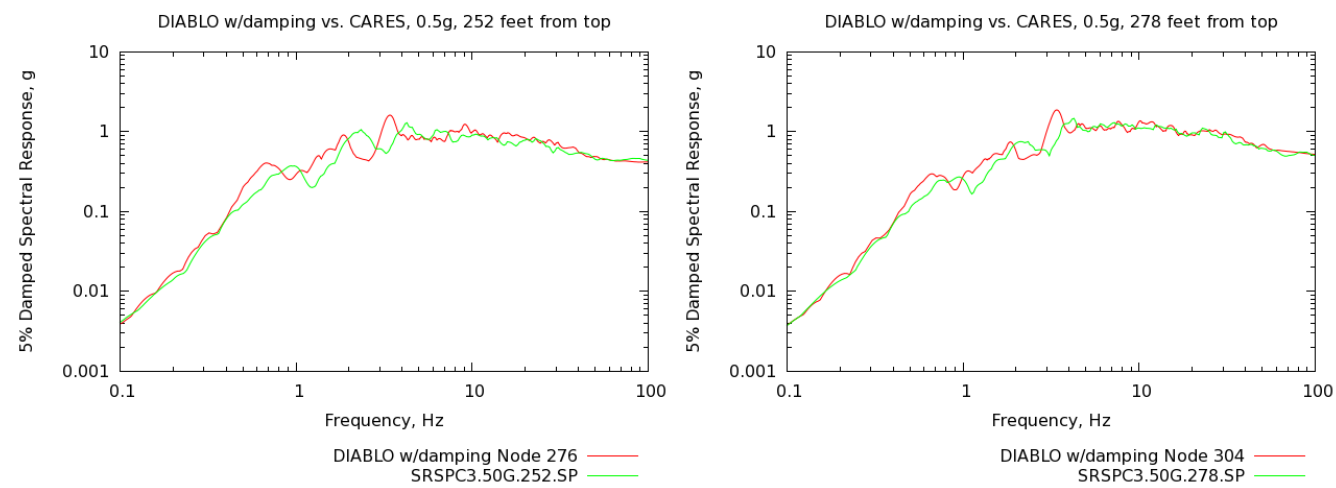

Figure 89 - 1D Soil column, $0.5 \mathrm{~g}$ earthquake, 5\% Response Spectra: DIABLO with viscous damping(red) versus CARES (green), distance 252 and $278 \mathrm{ft}$ from top surface
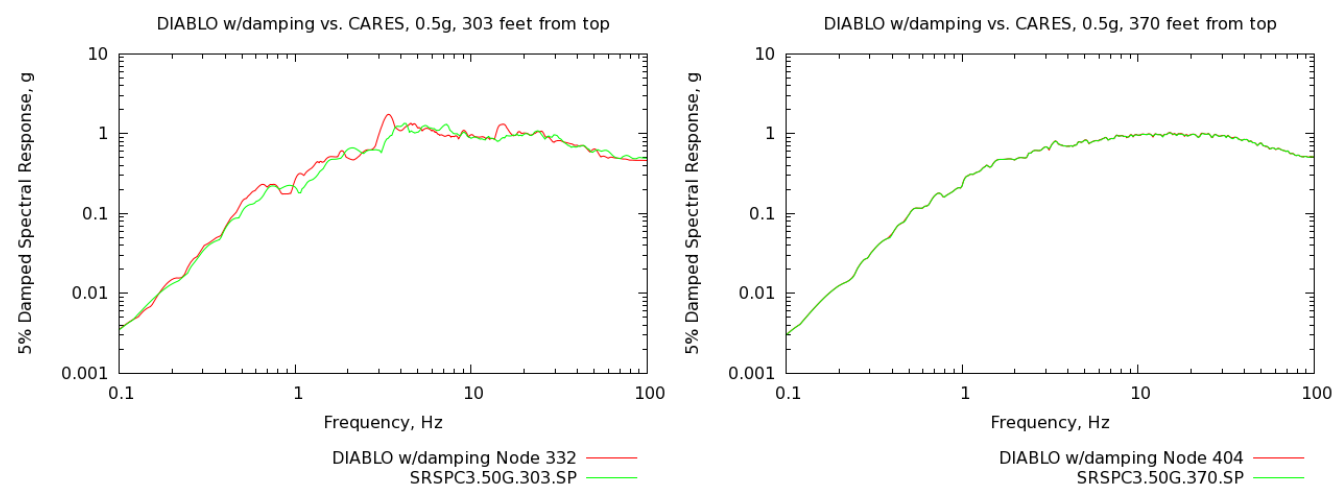

Figure 90 - 1D Soil column, 0.5g earthquake, 5\% Response Spectra: DIABLO with viscous damping(red) versus CARES (green), distance 303 and $370 \mathrm{ft}$ from top surface 


\section{A.4 One-D Soil Column, CARES 0.9g versus DIABLO with added viscous damping}
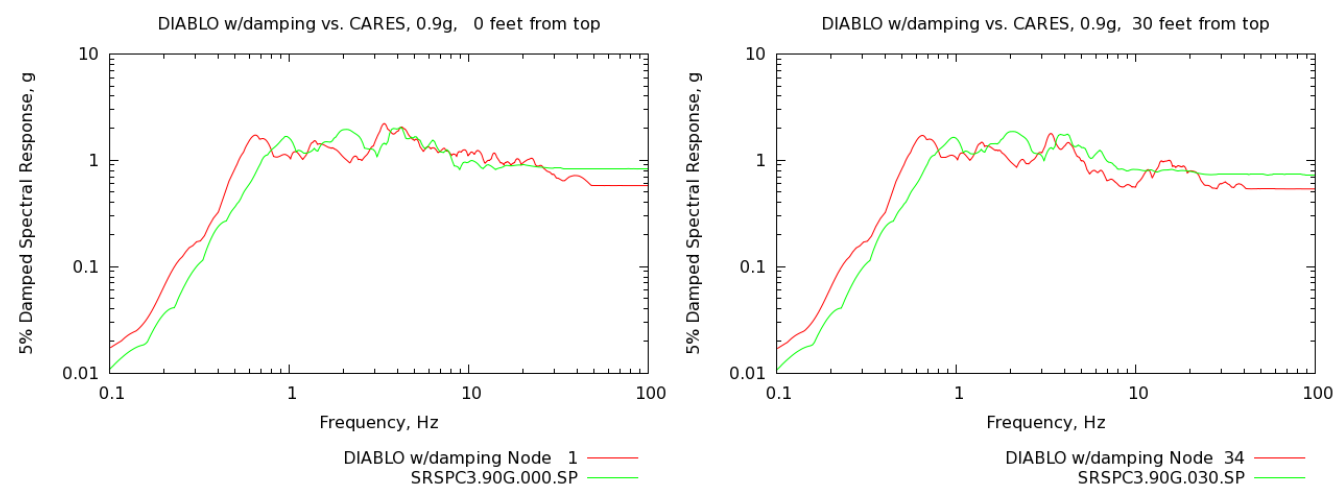

Figure 91 - 1D Soil column, 0.9g earthquake, 5\% Response Spectra: DIABLO with viscous damping(red) versus CARES (green), distance 0 and $30 \mathrm{ft}$ from top surface
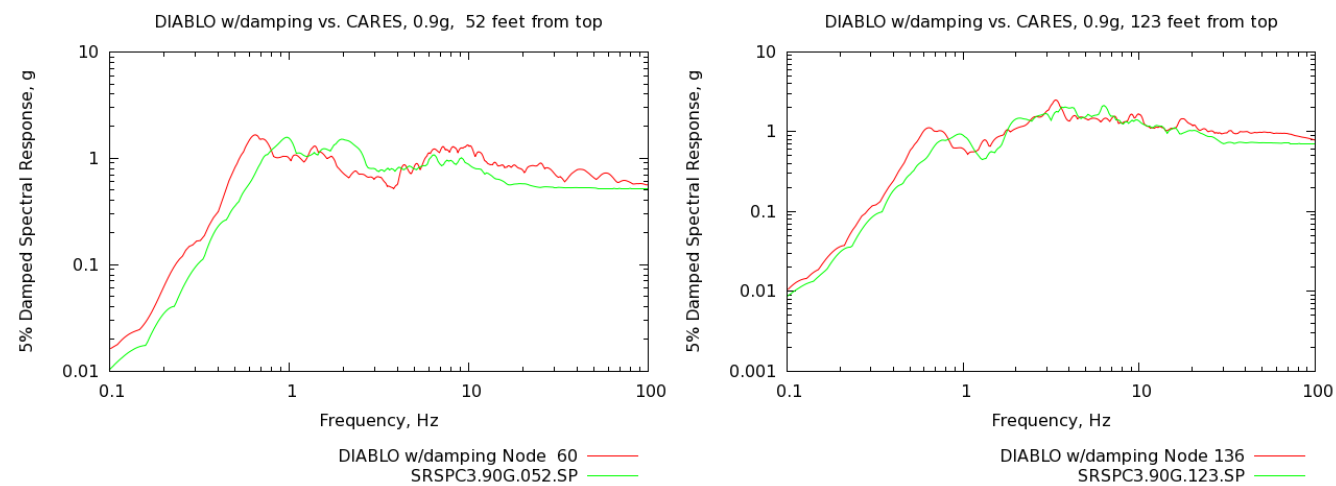

Figure 92 - 1D Soil column, 0.9g earthquake, 5\% Response Spectra: DIABLO with viscous damping(red) versus CARES (green), distance 052 and $123 \mathrm{ft}$ from top surface
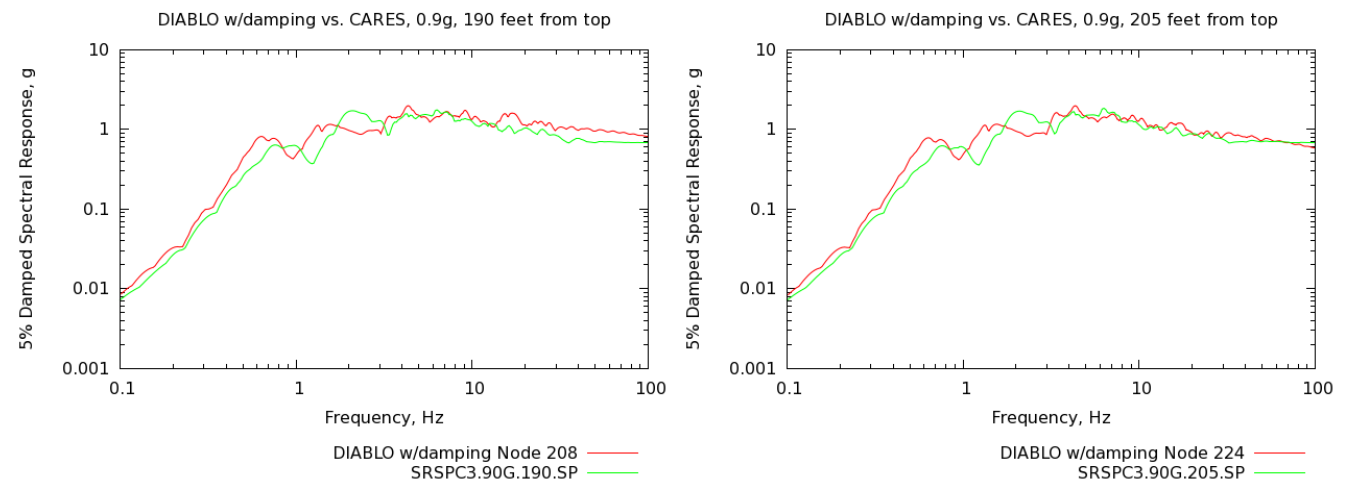

Figure 93 - 1D Soil column, 0.9g earthquake, 5\% Response Spectra: DIABLO with viscous damping(red) versus CARES (green), distance 190 and $205 \mathrm{ft}$ from top surface 

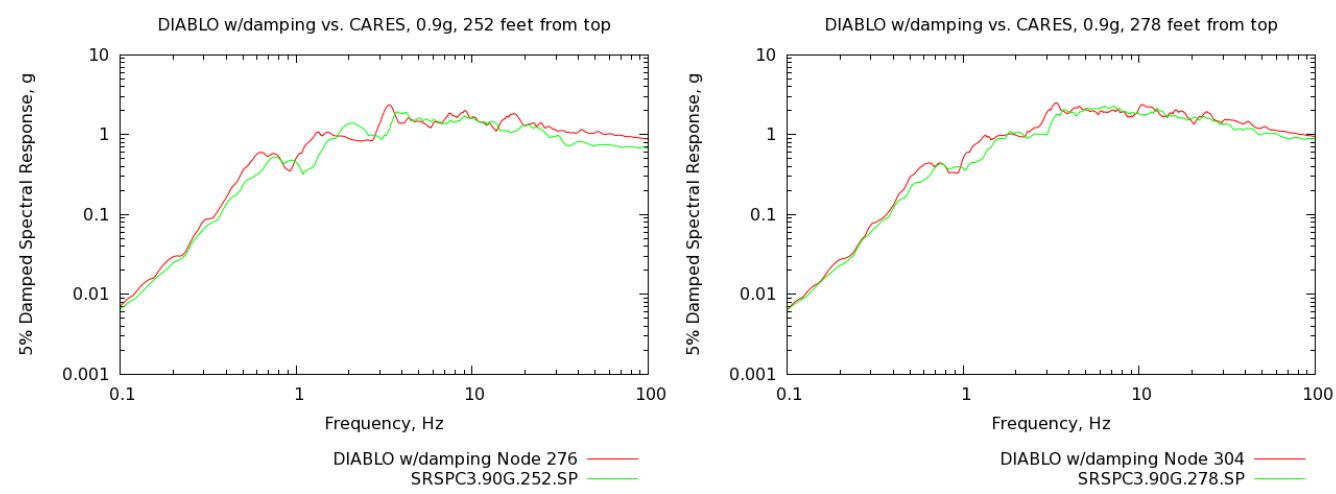

Figure 94 - 1D Soil column, 0.9g earthquake, 5\% Response Spectra: DIABLO with viscous damping(red) versus CARES (green), distance 252 and $278 \mathrm{ft}$ from top surface
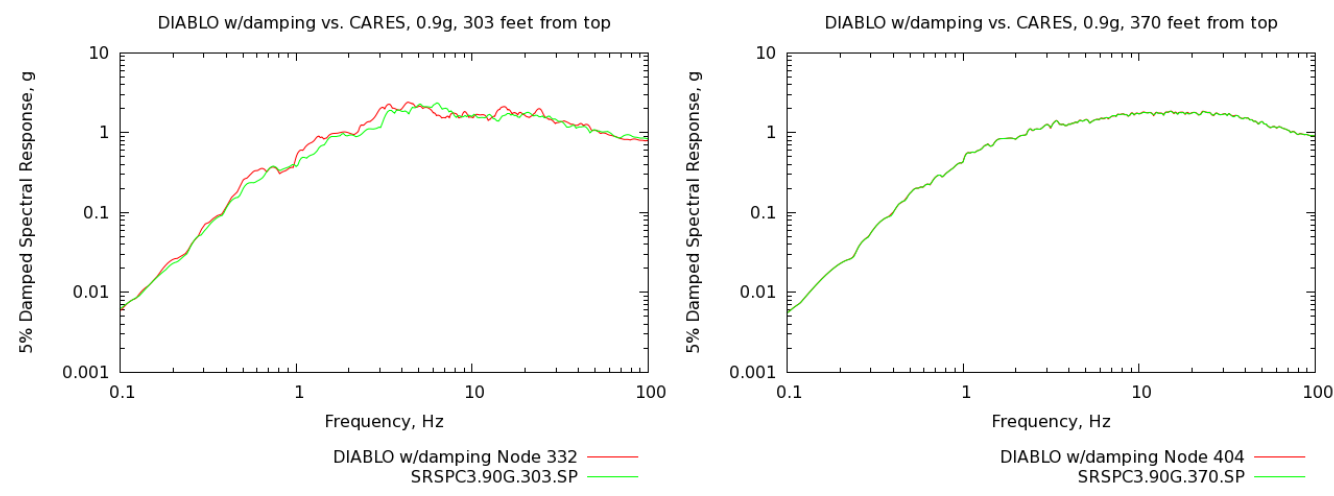

Figure 95 - 1D Soil column, 0.9g earthquake, 5\% Response Spectra: DIABLO with viscous damping(red) versus CARES (green), distance 303 and $370 \mathrm{ft}$ from top surface 
Appendix B: SASSI versus DIABLO Response Spectra Comparison Data
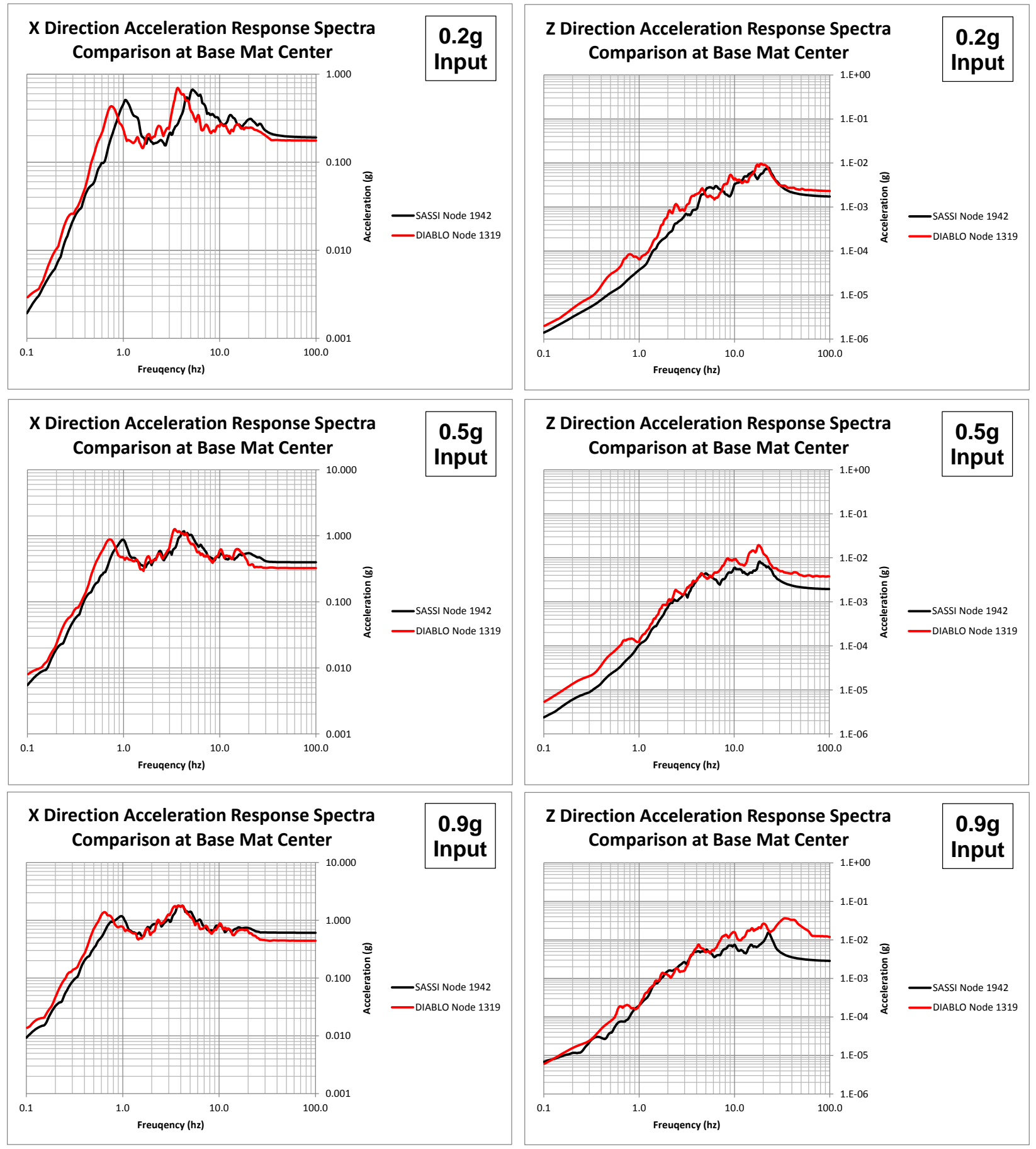

Figure 96 - Response Spectra Comparison DIABLO (red) versus SASSI (black), Basemat Center 

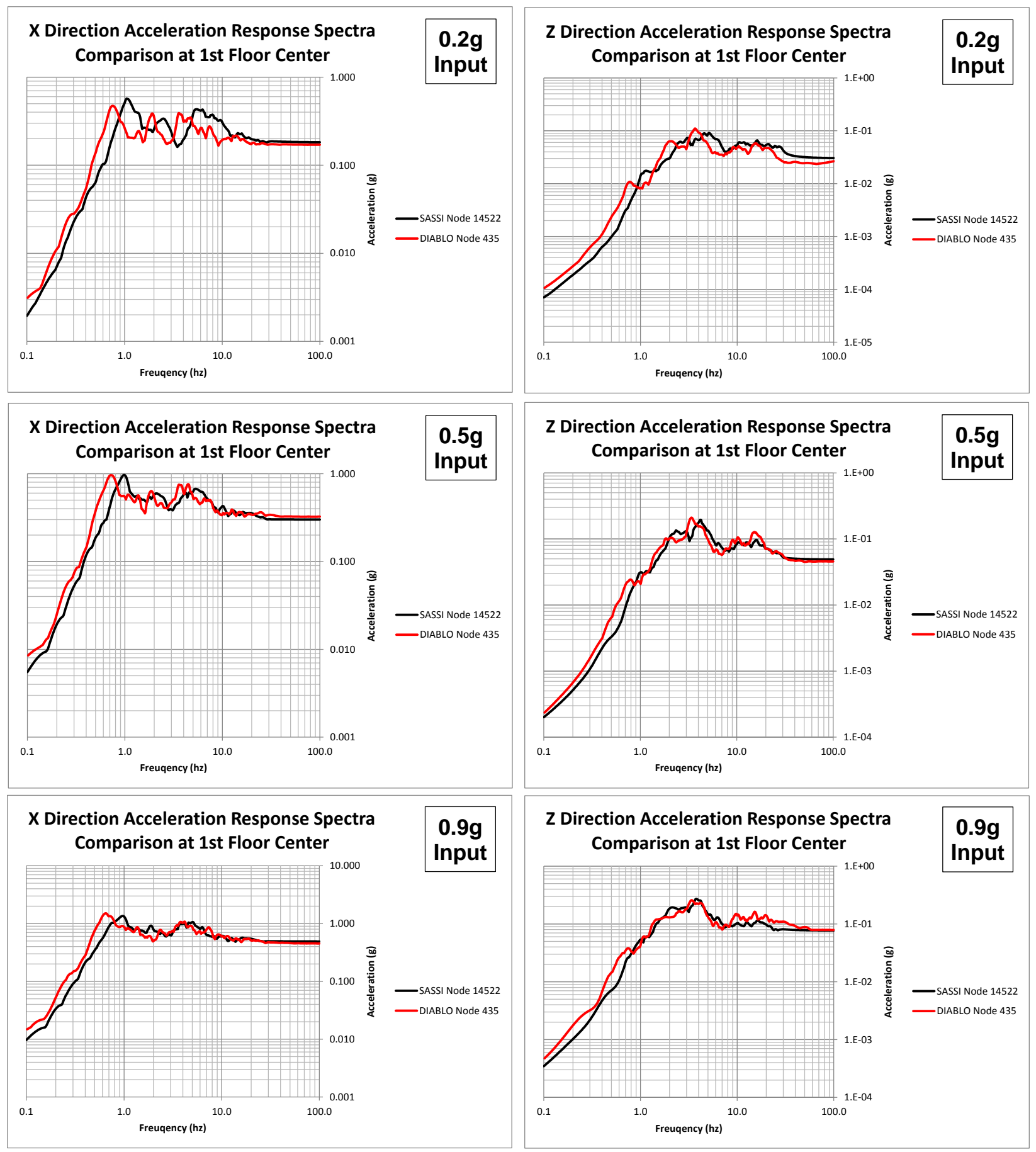

Figure 97 - Response Spectra Comparison, DIABLO (red) versus SASSI (black), First Floor Center 

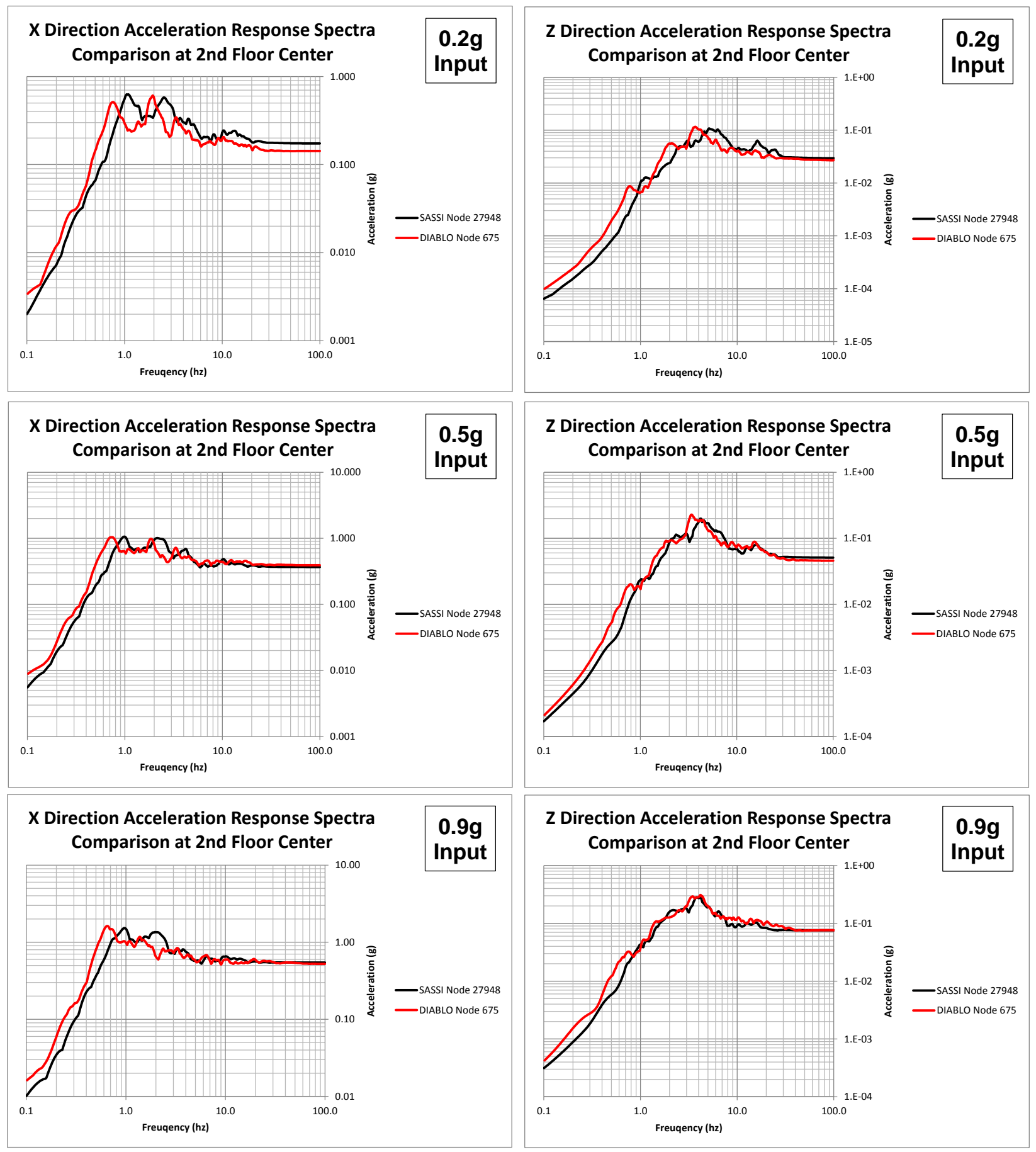

Figure 98 - Response Spectra Comparison, DIABLO (red) versus SASSI (black), 2nd Floor Center 

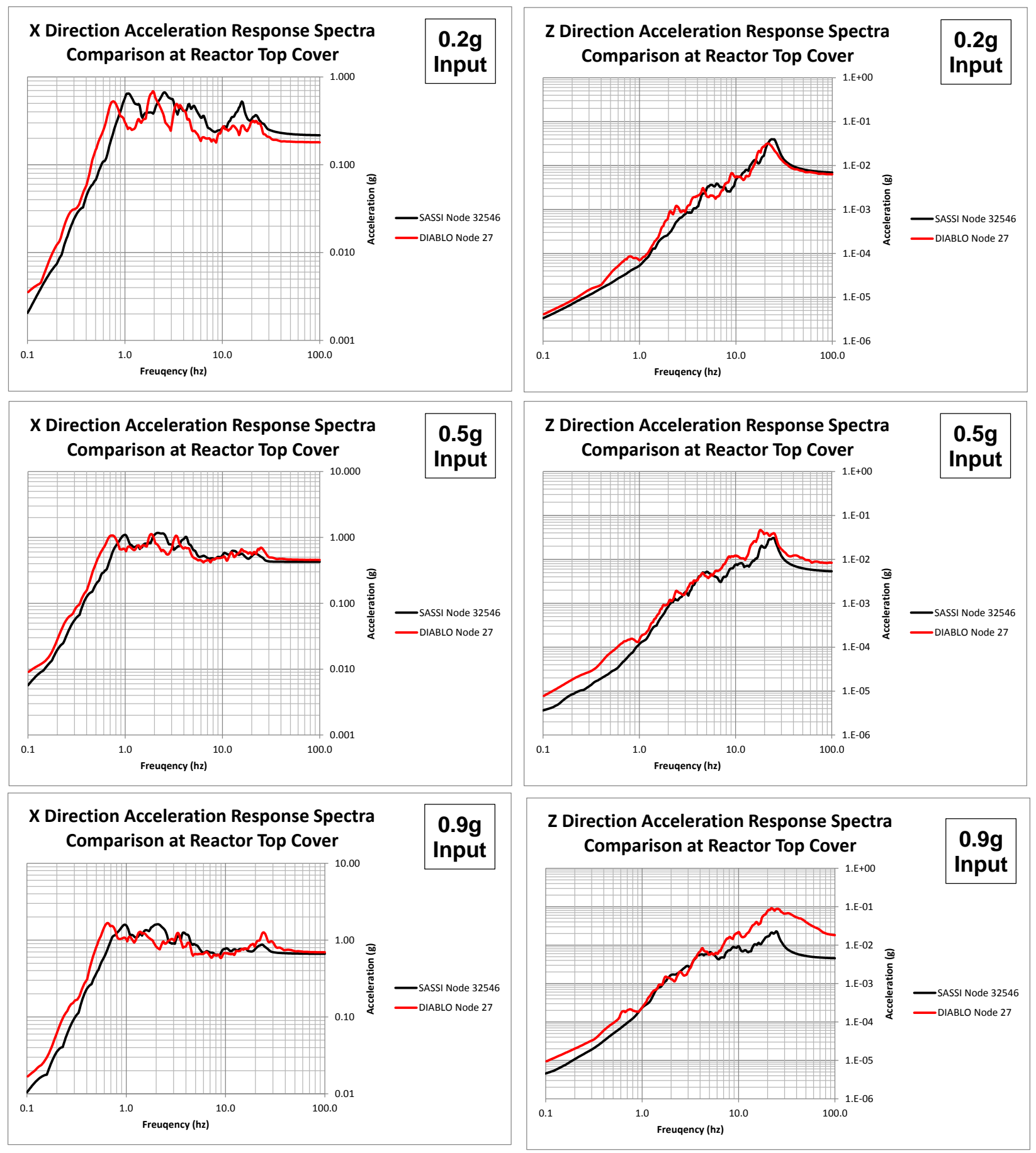

Figure 99 - Response Spectra Comparison, DIABLO (red) versus SASSI (black), Reactor Top Cover 

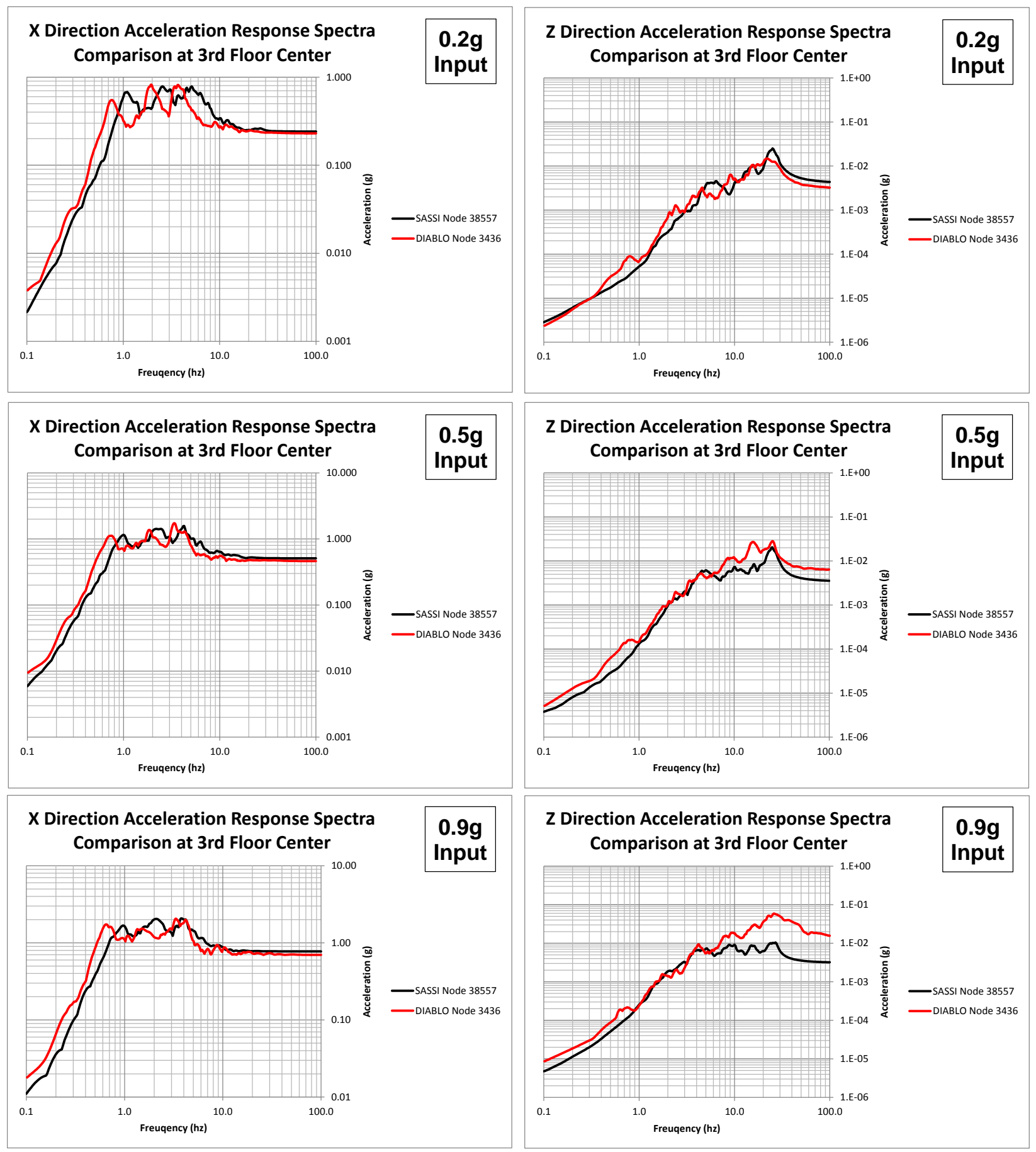

Figure 100 - Response Spectra Comparison, DIABLO (red) versus SASSI (black), 3rd Floor Center 

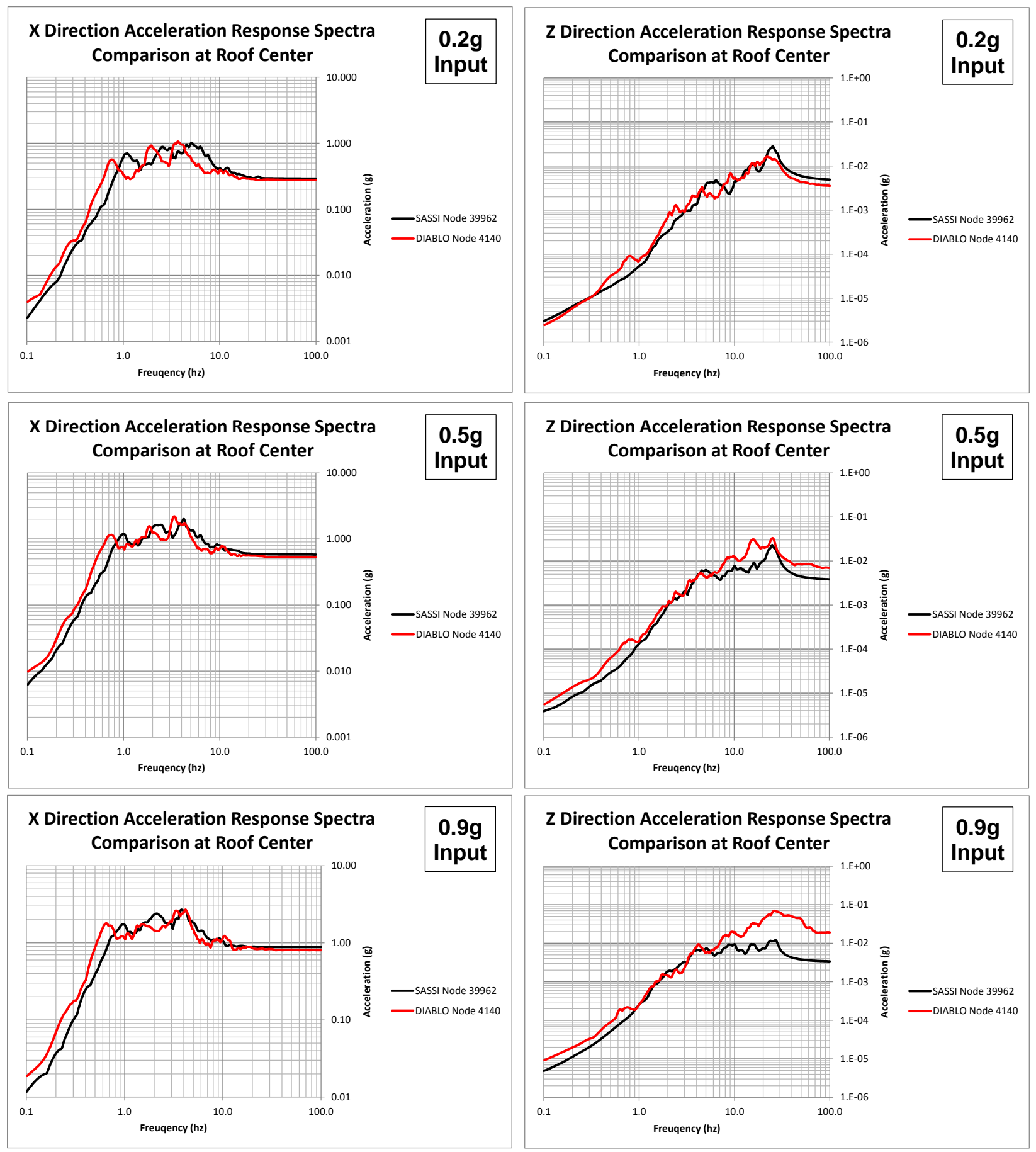

Figure 101 - Response Spectra Comparison, DIABLO (red) versus SASSI (black), Roof Center 

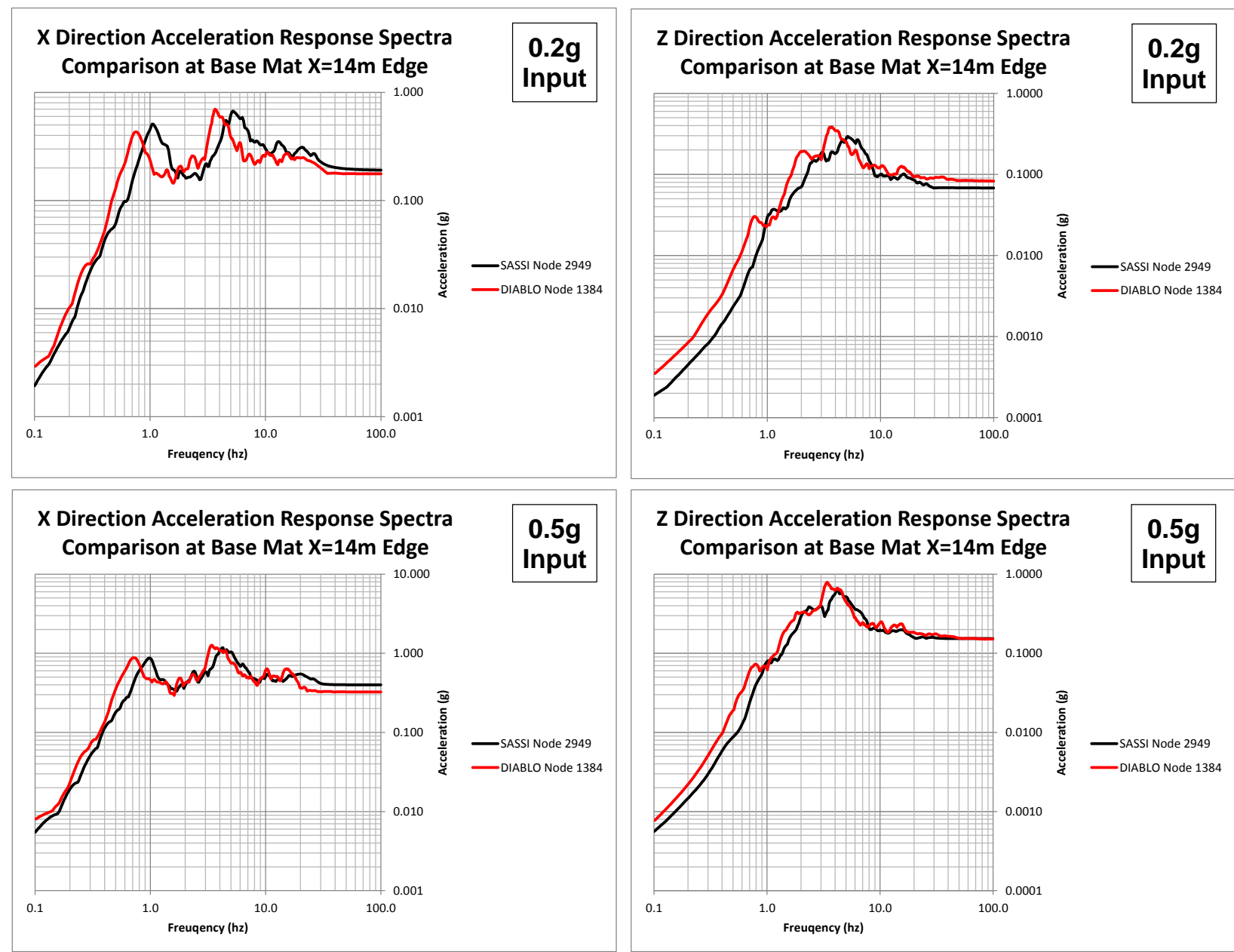

\section{Z Direction Acceleration Response Spectra Comparison at Base Mat $\mathrm{X}=14 \mathrm{~m}$ Edge}
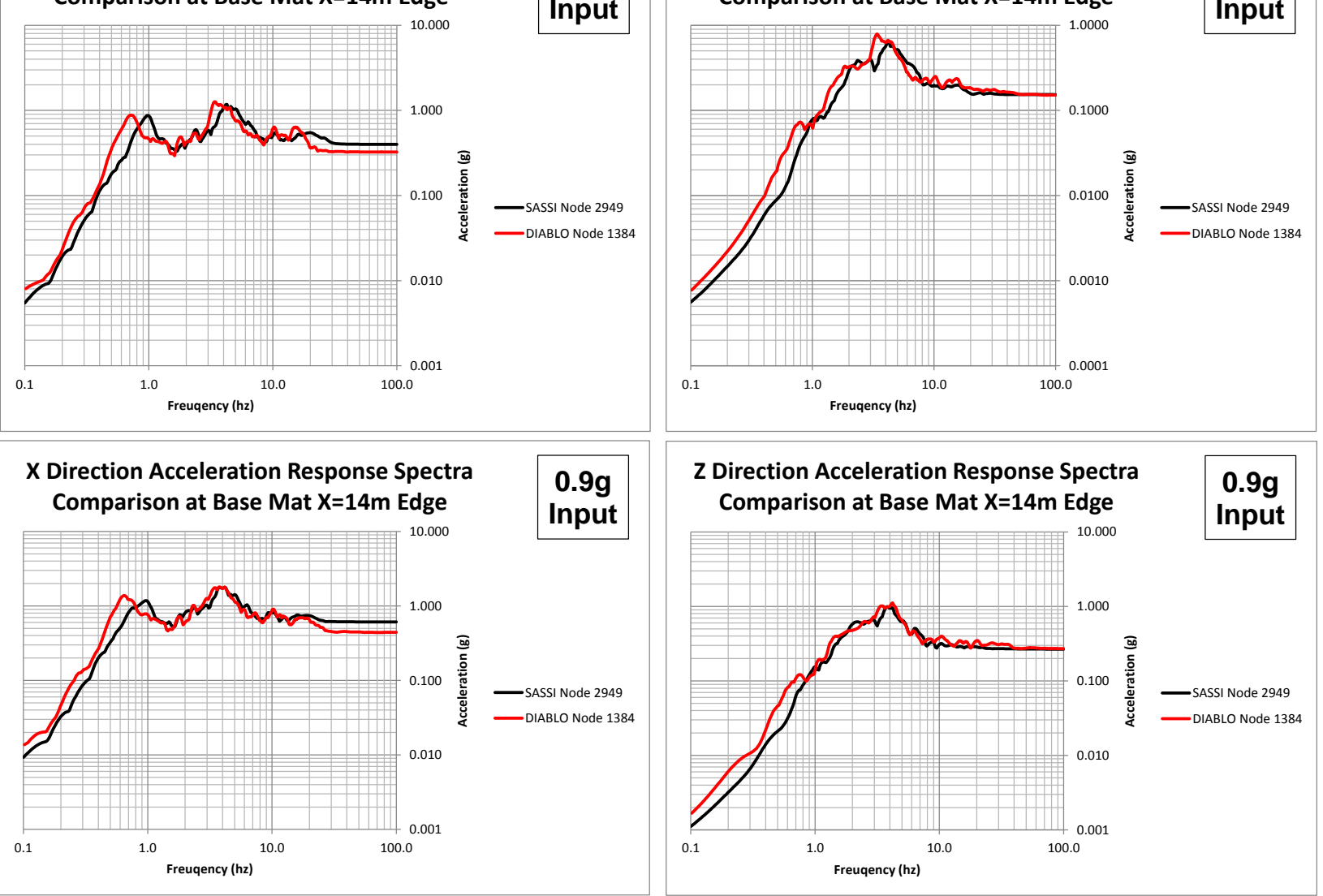

Figure 102 - Response Spectra Comparison, DIABLO (red) versus SASSI (black), BaseMat X=14 Edge 

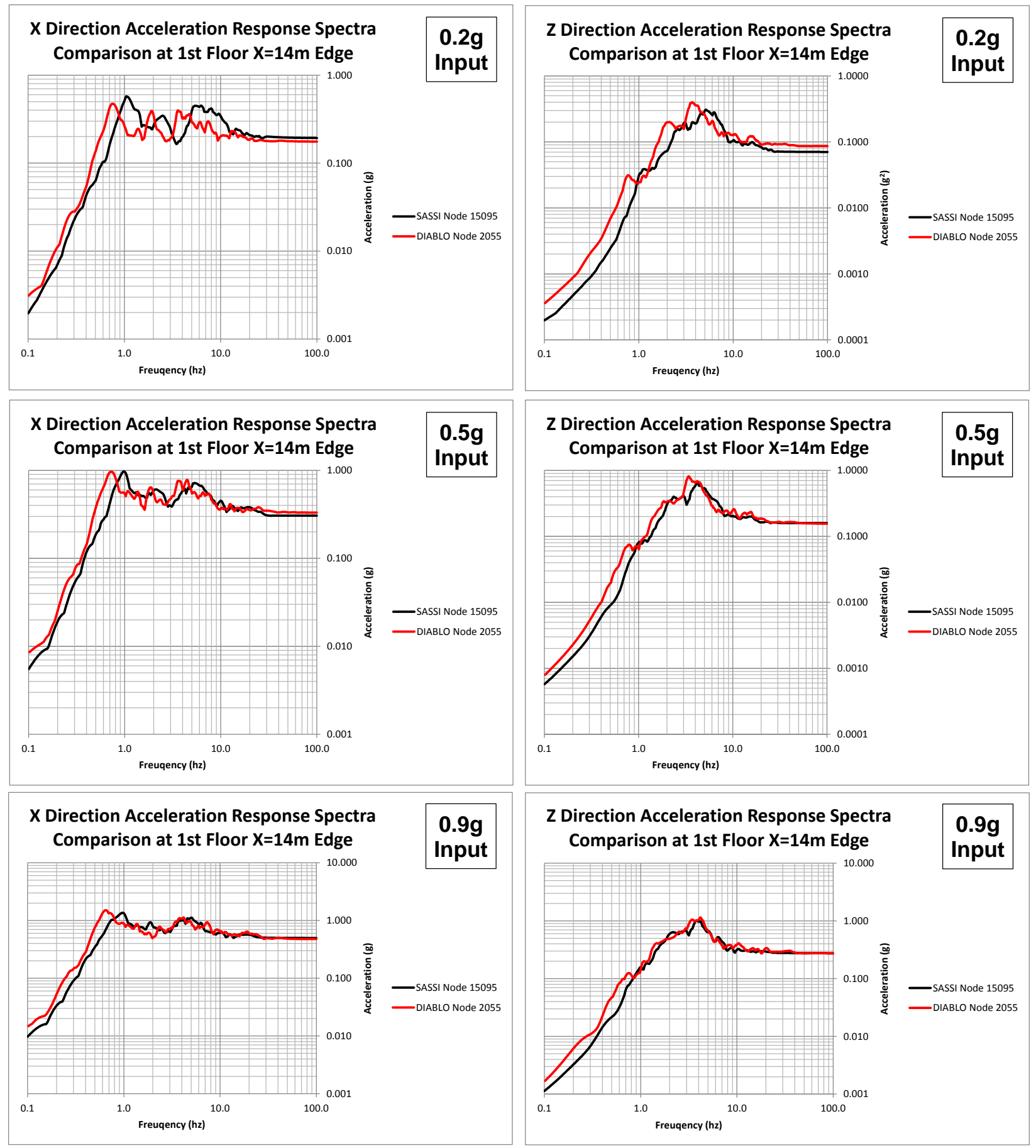

Figure 103 - Response Spectra Comparison, DIABLO (red) versus SASSI (black), First Floor X=14 Edge 

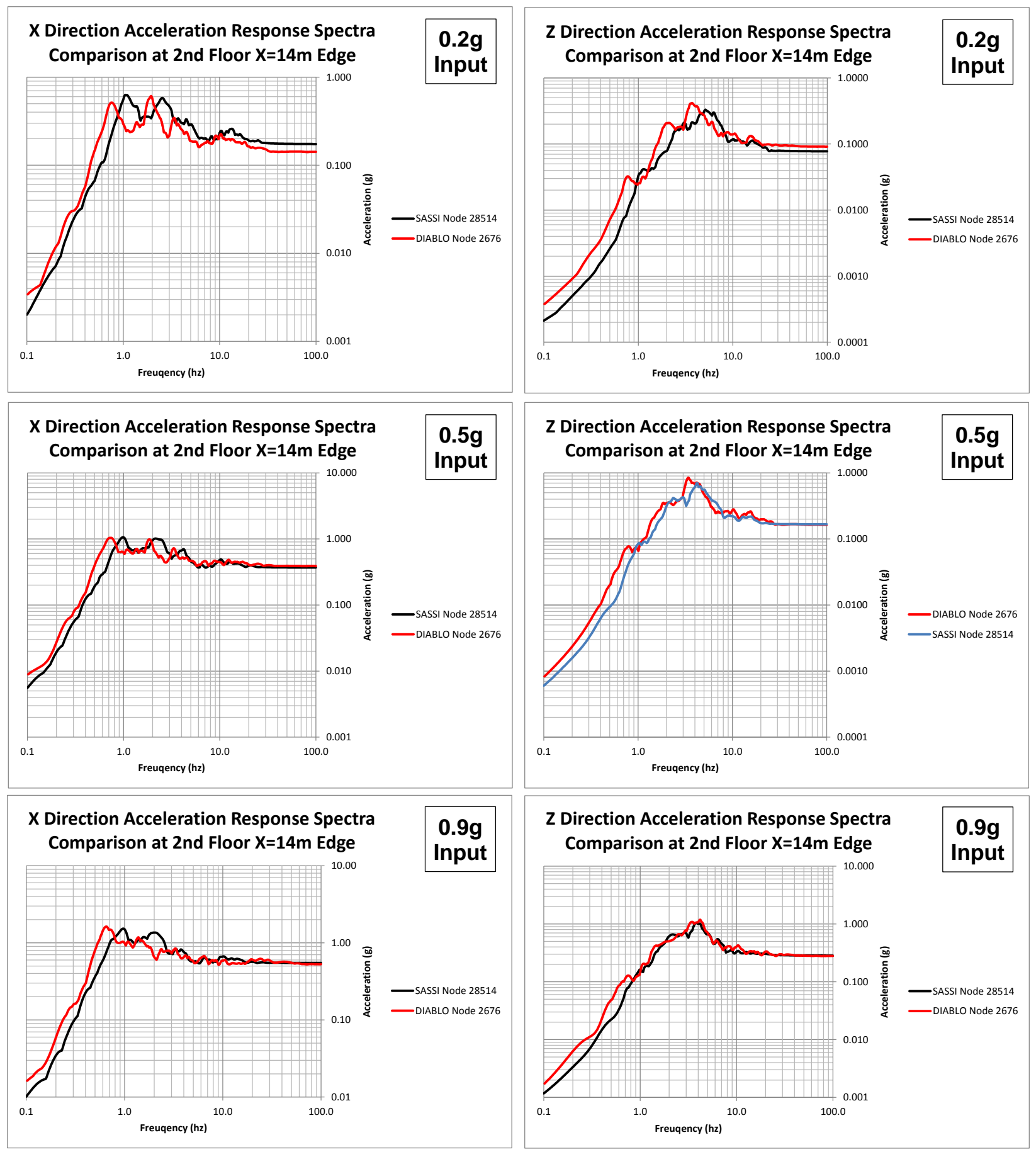

Figure 104 - Response Spectra Comparison, DIABLO (red) versus SASSI (black), 2nd Floor X=14 Edge 

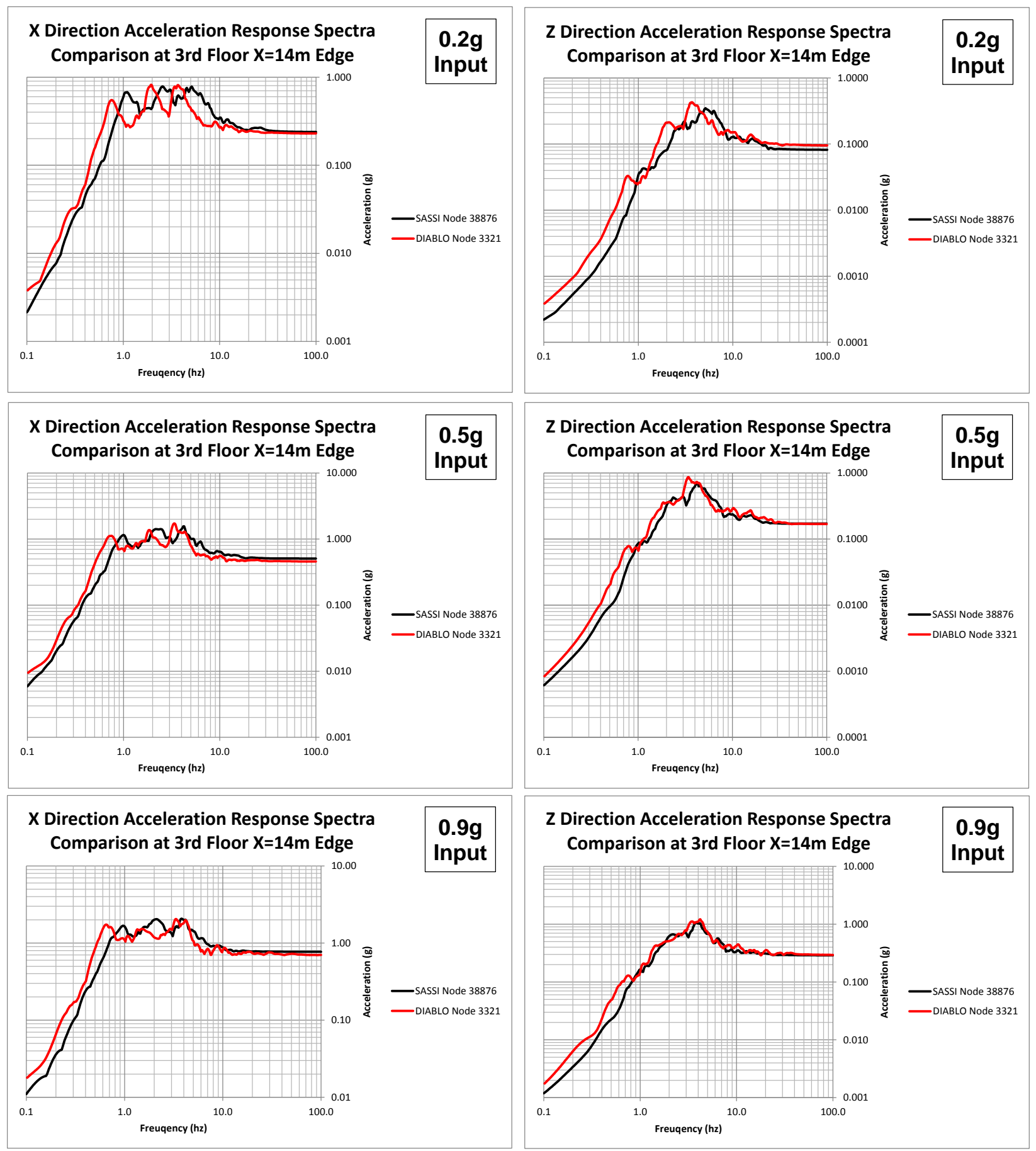

Figure 105 - Response Spectra Comparison, DIABLO (red) versus SASSI (black), 3rd Floor X=14 Edge 

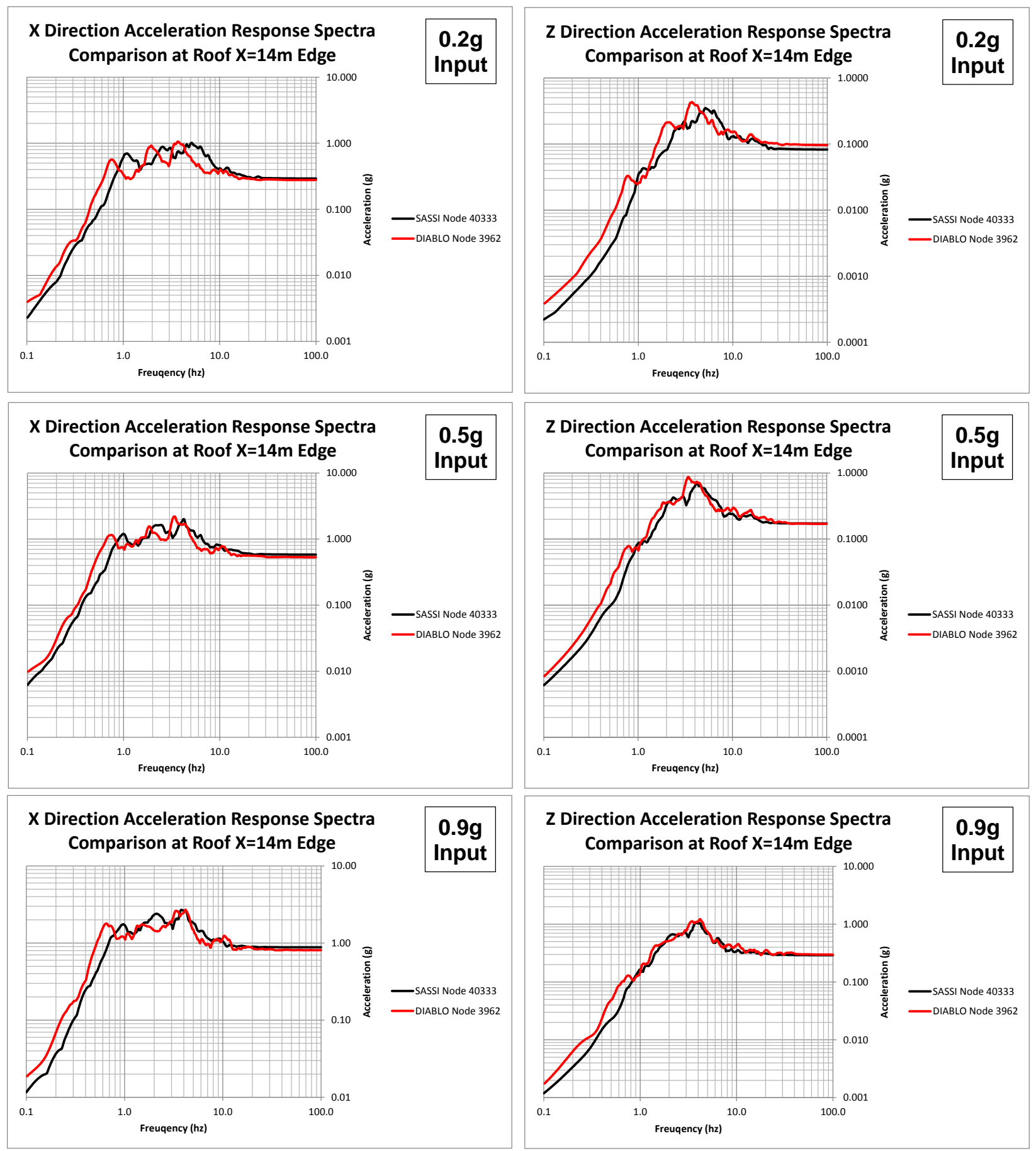

Figure 106 - Response Spectra Comparison, DIABLO (red) versus SASSI (black), Roof X=14 Edge 

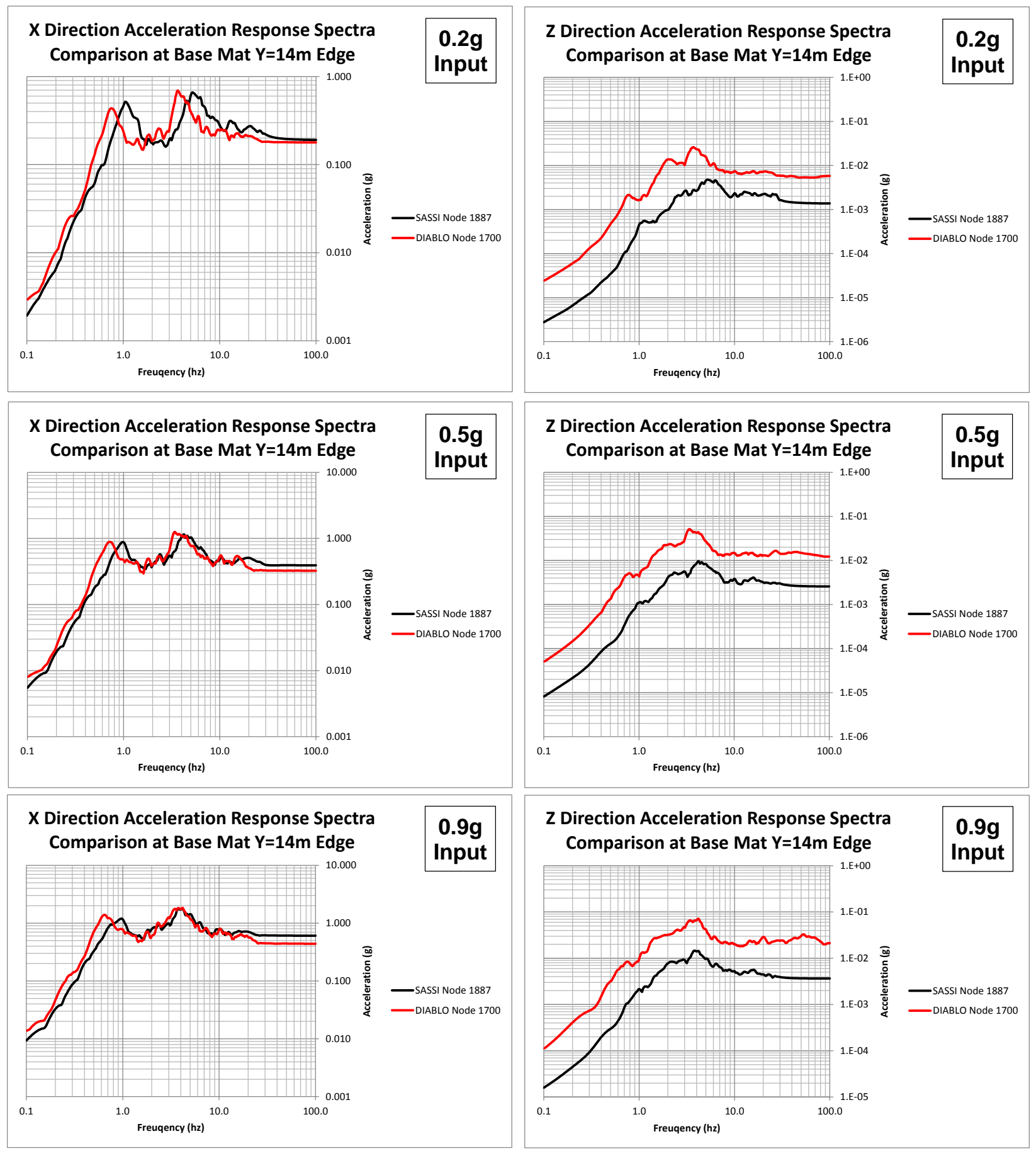

Figure 107 - Response Spectra Comparison, DIABLO (red) versus SASSI (black), BaseMat Y=14 Edge 

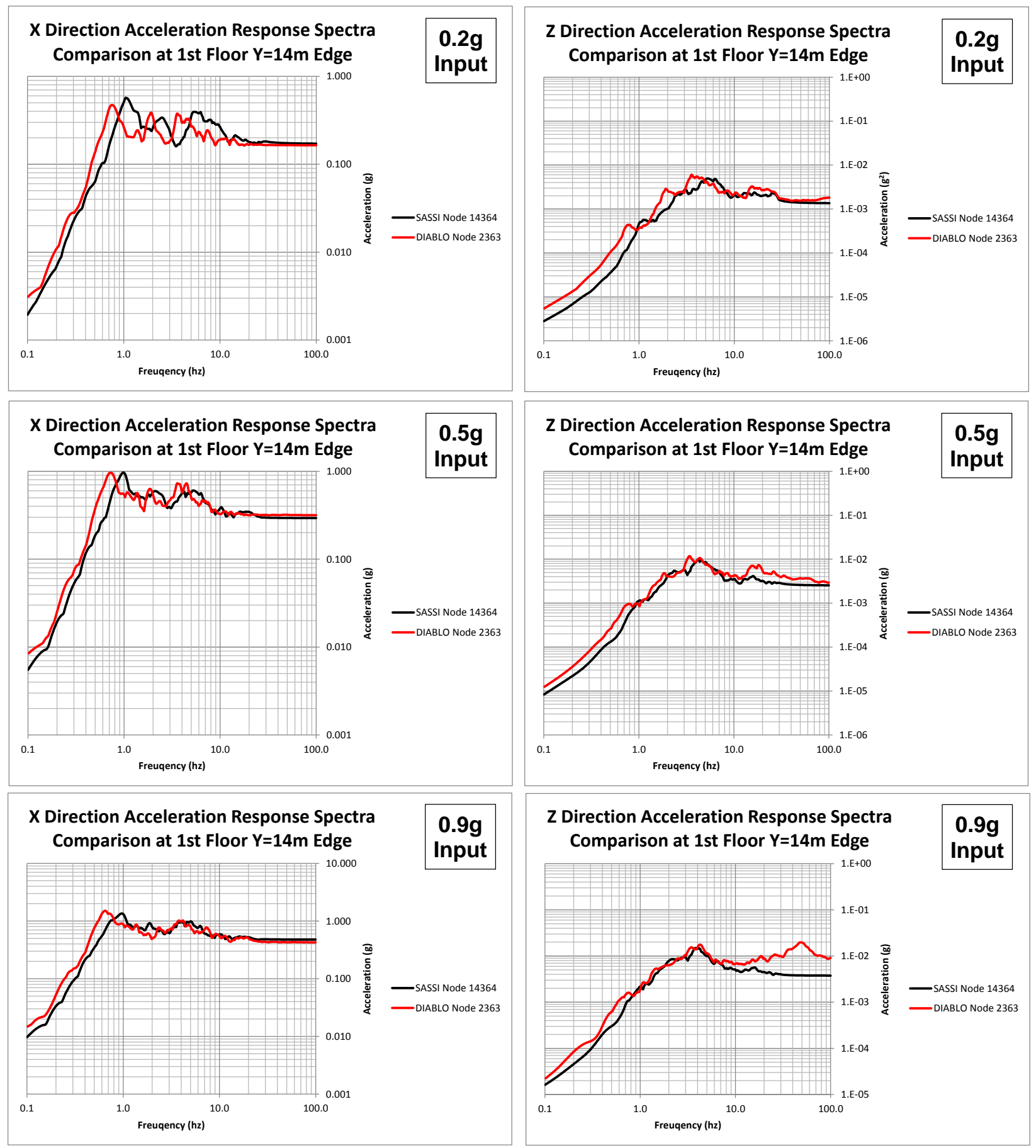

Figure 108 - Response Spectra Comparison, DIABLO (red) versus SASSI (black), 1st Floor Y=14 Edge 

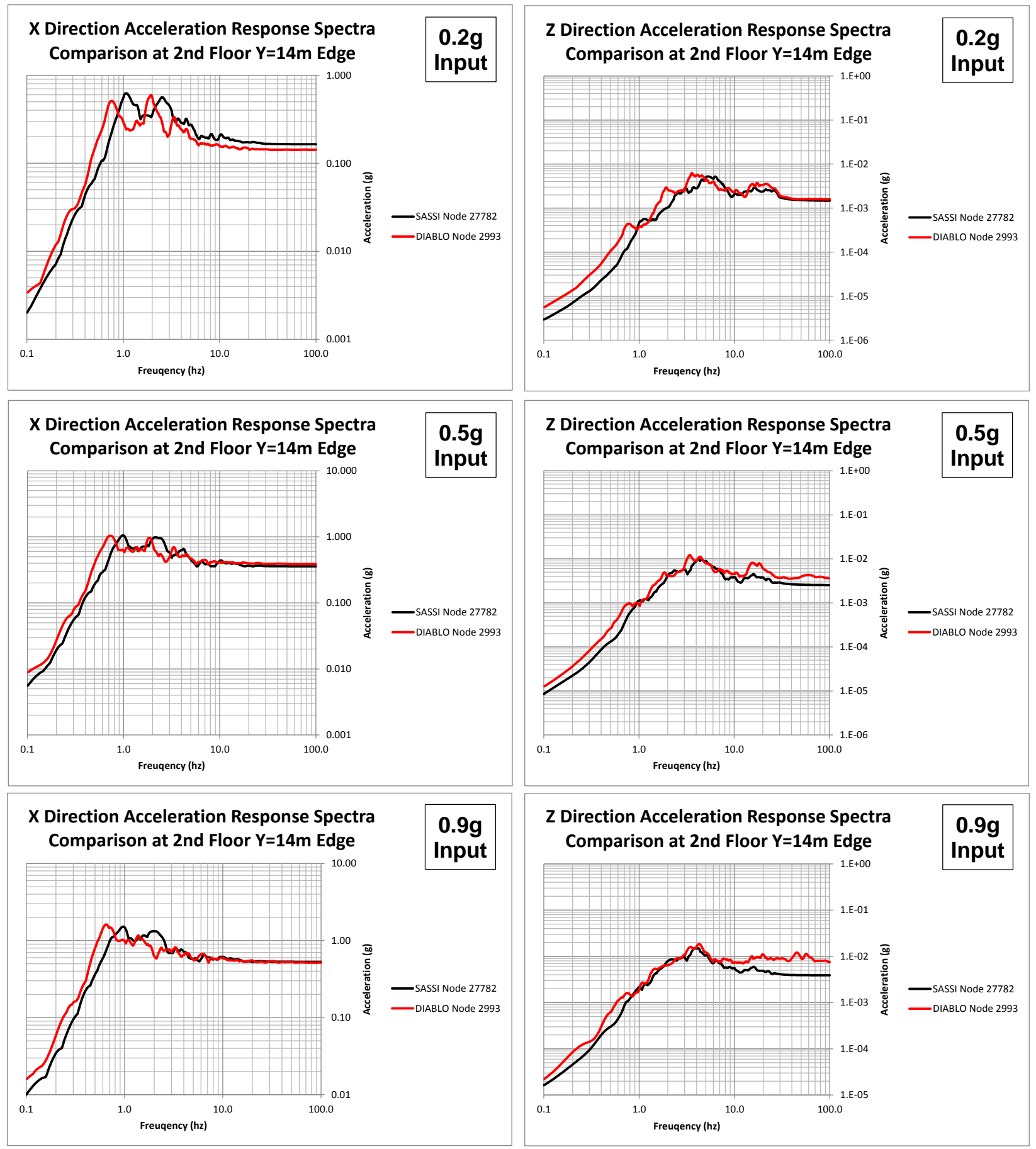

Figure 109 - Response Spectra Comparison, DIABLO (red) versus SASSI (black), 2nd Floor Y=14 Edge 

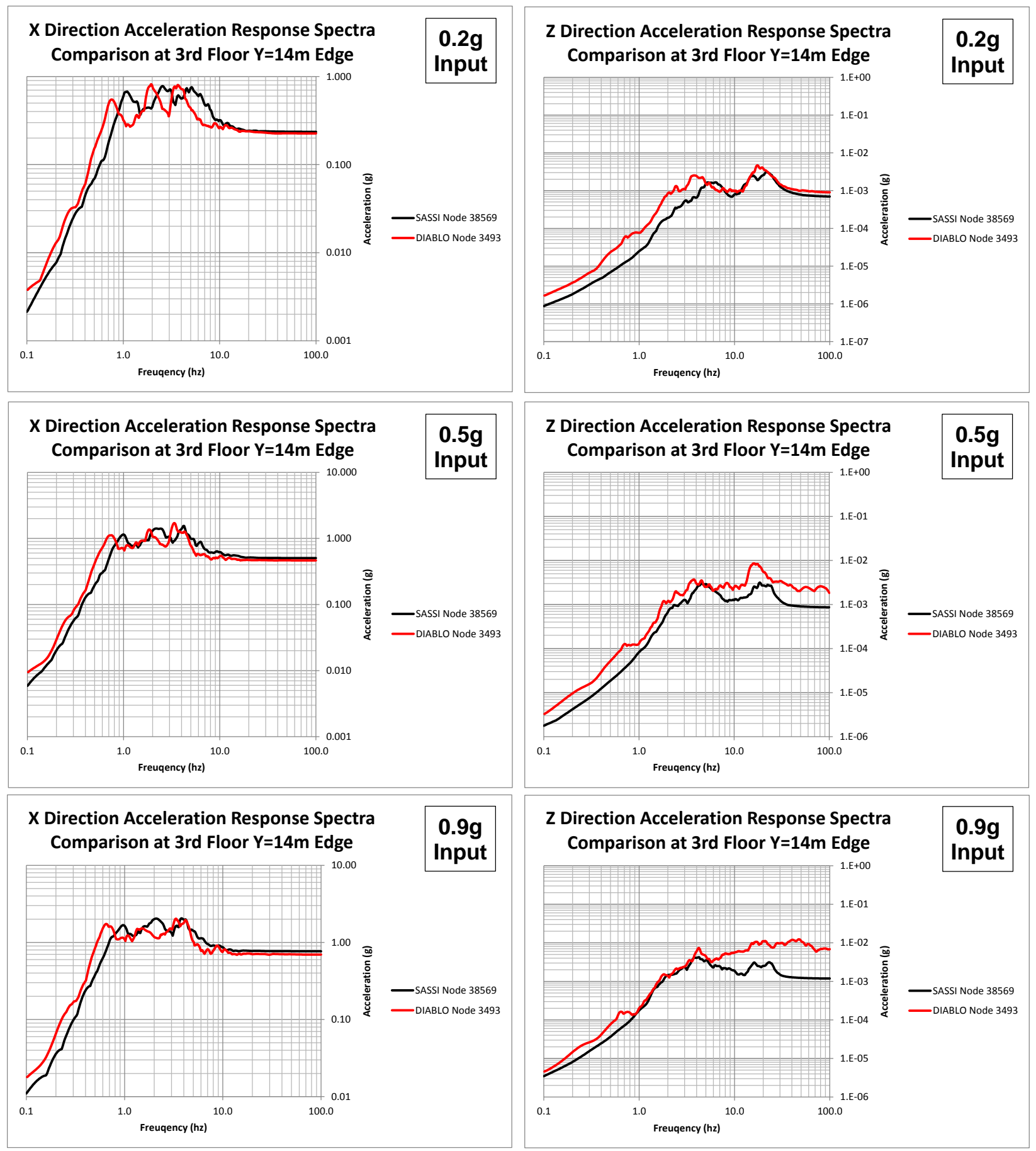

Figure 110 - Response Spectra Comparison, DIABLO (red) versus SASSI (black), 3rd Floor Y=14 Edge 

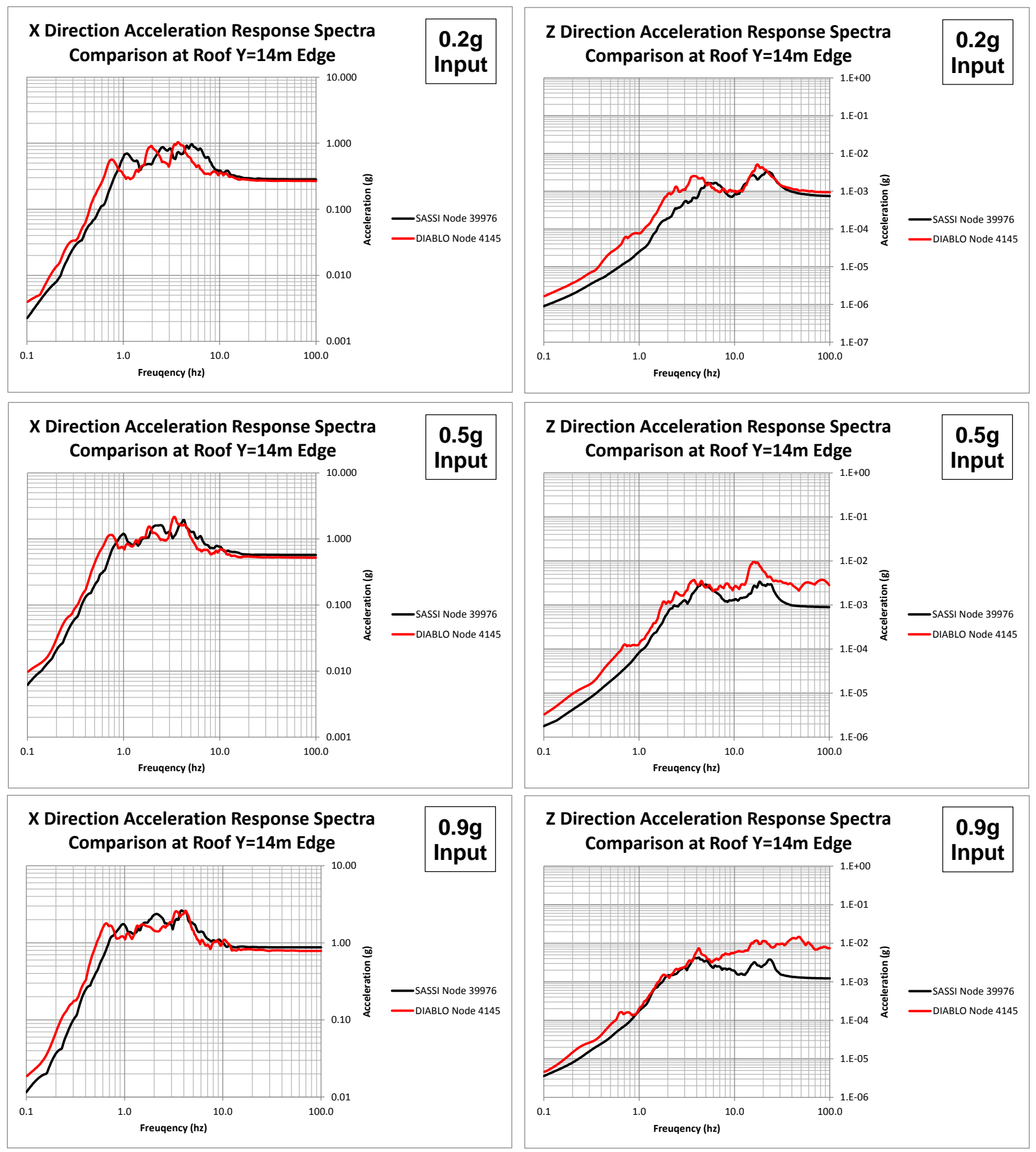

Figure 111 - Response Spectra Comparison, DIABLO (red) versus SASSI (black), Roof Y=14 Edge 